\title{
Geochemical Modeling of ILAW Lysimeter Water Extracts
}

\section{December 2014}

KJ Cantrell 


\title{
DISCLAIMER
}

This report was prepared as an account of work sponsored by an agency of the United States Government. Neither the United States Government nor any agency thereof, nor Battelle Memorial Institute, nor any of their employees, makes any warranty, express or implied, or assumes any legal liability or responsibility for the accuracy, completeness, or usefulness of any information, apparatus, product, or process disclosed, or represents that its use would not infringe privately owned rights. Reference herein to any specific commercial product, process, or service by trade name, trademark, manufacturer, or otherwise does not necessarily constitute or imply its endorsement, recommendation, or favoring by the United States Government or any agency thereof, or Battelle Memorial Institute. The views and opinions of authors expressed herein do not necessarily state or reflect those of the United States Government or any agency thereof.

\author{
PACIFIC NORTHWEST NATIONAL LABORATORY \\ operated by \\ BATTELLE \\ for the \\ UNITED STATES DEPARTMENT OF ENERGY \\ under Contract DE-AC05-76RL01830
}

Printed in the United States of America

Available to DOE and DOE contractors from the

Office of Scientific and Technical Information,

P.O. Box 62, Oak Ridge, TN 37831-0062;

ph: (865) 576-8401

fax: $(865) 576-5728$

email: reports@adonis.osti.gov

\author{
Available to the public from the National Technical Information Service \\ 5301 Shawnee Rd., Alexandria, VA 22312 \\ ph: (800) 553-NTIS (6847) \\ email: orders $a$ ntis.gov $<$ http://www.ntis.gov/about/form.aspx> \\ Online ordering: http://www.ntis.gov
}

This document was printed on recycled paper. 
PNNL-23693

RPT-66309-002, Rev. 0

\section{Geochemical Modeling of ILAW Lysimeter Water Extracts}

KJ Cantrell

December 2014

Prepared for

the U.S. Department of Energy

under Contract DE-AC05-76RL01830

Pacific Northwest National Laboratory

Richland, Washington 99352 



\section{Executive Summary}

Geochemical modeling results of water extracts from simulated immobilized low-activity waste (ILAW) glasses, placed in lysimeters for eight years suggest that the secondary phase reaction network developed using product consistency test (PCT) results at $90{ }^{\circ} \mathrm{C}$ may need to be modified for field conditions. For sediment samples that had been collected from near the glass samples, the impact of glass corrosion could be readily observed based upon the $\mathrm{pH}$ of their water extracts. For unimpacted sediments the $\mathrm{pH}$ ranged from 7.88 to 8.11 with an average of 8.04. Sediments that had observable impacts from glass corrosion exhibited elevated $\mathrm{pH}$ values (as high as 9.97). For lysimeter sediment samples that appear to have been impacted by glass corrosion to the greatest extent, saturation indices determined for analcime, calcite, and chalcedony in the 1:1 water extracts were near equilibrium and were consistent with the secondary phase reaction network developed using PCT results at $90{ }^{\circ} \mathrm{C}$. $\mathrm{Fe}(\mathrm{OH})_{3}(\mathrm{~s})$ also appears to be essentially at equilibrium in extracts impacted by glass corrosion, but with a solubility product $\left(\log \mathrm{K}_{\mathrm{sp}}\right)$ that is approximately 2.13 units lower than that used in the secondary phase reaction network developed using PCT results at $90{ }^{\circ} \mathrm{C}$. The solubilities of $\mathrm{TiO}_{2}(\mathrm{am})$ and $\mathrm{ZrO}_{2}(\mathrm{am})[(\mathrm{am})$ indicates an amorphous phase] also appear to be much lower than that assumed in the secondary phase reaction network developed using PCT results at $90{ }^{\circ} \mathrm{C}$. The extent that the solubility of $\mathrm{TiO}_{2}(\mathrm{am})$ and $\mathrm{ZrO}_{2}(\mathrm{am})$ were reduced relative to that assumed in the secondary phase reaction network developed using PCT results at $90^{\circ} \mathrm{C}$ could not be quantified because the concentrations of $\mathrm{Ti}$ and $\mathrm{Zr}$ in the extracts were below the estimated quantification limit. Gibbsite was consistently highly oversaturated in the extract while dawsonite was at or near equilibrium. This suggests that dawsonite might be a more suitable phase for the secondary phase reaction network than gibbsite under field conditions. This may be due to the availability of carbonate that exists in the Hanford sediments as calcite. A significant source of carbonate was not available in the PCTs and this may account for why this phase did not appear in the PCTs. Sepiolite was consistently highly undersaturated, suggesting that another phase controls the solubility of magnesium. For samples that were most impacted by the effects of glass corrosion, magnesite appears to control glass corrosion. For samples that show less impact from glass corrosion, clinochlore-7A or saponite-Mg appears to control the magnesium concentrations. For zinc, it appears that zincite is a better candidate than $\mathrm{Zn}(\mathrm{OH})_{2}-\gamma$ for controlling zinc concentrations in the extracts; however, in some samples all zinc phases considered were highly oversaturated. As a result the phase that controls zinc concentrations in the lysimeter extracts remains uncertain. 



\section{Acronyms and Abbreviations}

$\begin{array}{ll}\text { DOE } & \text { U.S. Department of Energy } \\ \text { EQL } & \text { estimated quantification limit } \\ \text { FIB } & \text { focused ion beam } \\ \text { FLTF } & \text { Field Lysimeter Test Facility } \\ \text { FY } & \text { fiscal year (October 1 through September 30; e.g., FY 2010 spans } \\ & \text { October 1, 2009 through September 30, 2010) } \\ \text { ICP-OES } & \text { inductively coupled plasma optical emission spectroscopy } \\ \text { IDF } & \text { Integrated Disposal Facility } \\ \text { IDF-PA } & \text { Integrated Disposal Facility performance assessment } \\ \text { ILAW } & \text { immobilized low-activity waste } \\ \text { LAW } & \text { low-activity waste } \\ \text { PA } & \text { performance assessment } \\ \text { PCT } & \text { product consistency test } \\ \text { PVC } & \text { polyvinyl chloride } \\ \text { PNNL } & \text { Pacific Northwest National Laboratory } \\ \text { QA } & \text { quality assurance } \\ \text { R\&D } & \text { research and development } \\ \text { RTL } & \text { Research Technology Laboratory } \\ \text { SEM } & \text { scanning electron microscopy } \\ \text { SEM-EDS } & \text { scanning electron microscopy-energy dispersive spectroscopy } \\ \text { SI } & \text { saturation index } \\ \text { STOMP } & \text { Subsurface Transport Over Multiple Phases } \\ \text { TEM-SAED } & \text { transmission electron microscopy-selected area electron diffraction } \\ \text { WRPS } & \text { Washington River Protection Solutions } \\ \text { WWFTP } & \text { WRPS Waste Form Testing Program } \\ \text { XRD } & \text { X-ray diffraction } \\ & \end{array}$





\section{Contents}

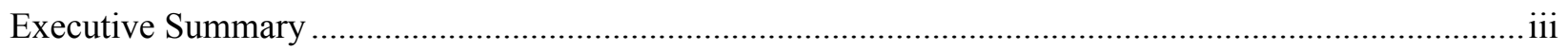

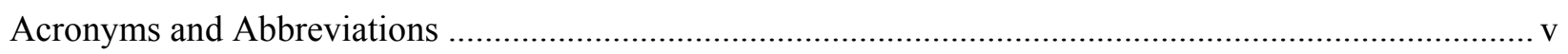

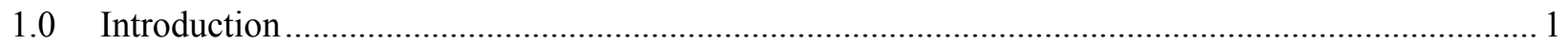

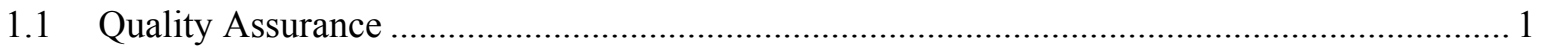

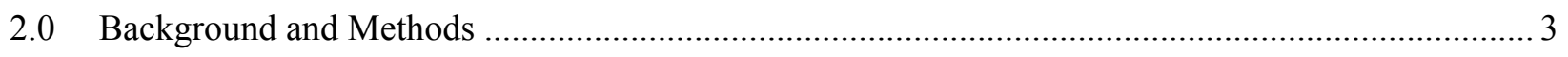

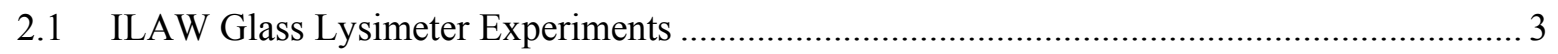

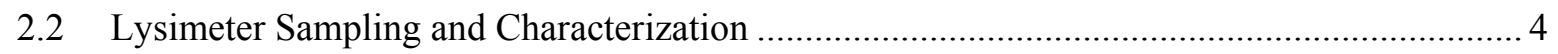

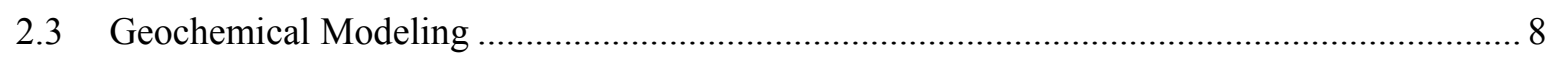

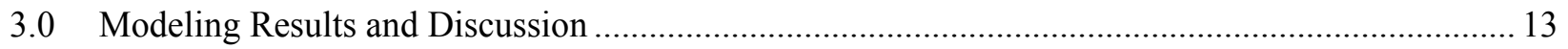

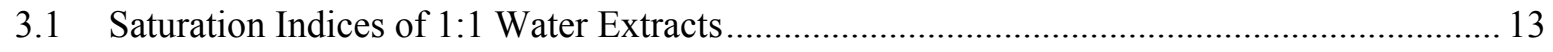

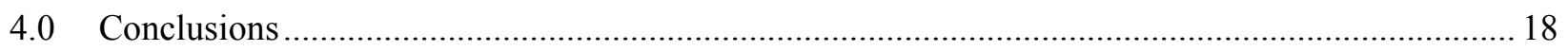

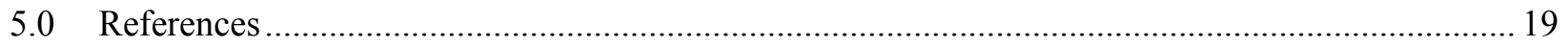

Appendix A - Geochemist's Workbench Output ................................................................................. A.1 


\section{Figures}

1. Surface of Field Lysimeter Test Facility Showing Several Lysimeters and a Side View Diagram of ILAW Glass Test Lysimeters Showing that Each Consisted of Two Groups of Three Glass Cylinders at Upper and Lower Levels ................................................................... 3

2. Glass Cylinders Being Buried in a Lysimeter at the Hanford Site................................................ 4

3. Side View Diagram of ILAW Glass Test Lysimeters Showing that Each Consisted of Two Groups of Three Glass Cylinders at Upper and Lower Levels .......................................................... 5

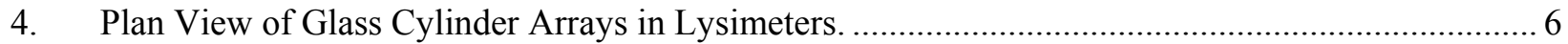

5. Field Photo of Lysimeter D-14 Showing Locations of Large-Diameter Cores Outside Cylinder Array and the Channel of a Small-Diameter Core between Cylinders .............................. 7

6. Photograph of Part of a Glass Cylinder from Lysimeter D-14 Showing the Process of Collection of Glass with Attached Sediment ................................................................................... 8

7. Lysimeter D-10 Glass and Sediment Core Locations …............................................................ 9

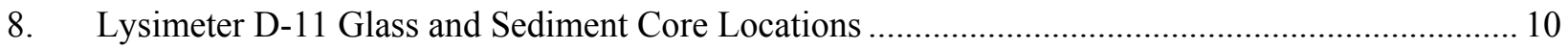

9. Lysimeter D-14 Glass and Sediment Core Locations ............................................................. 11

\section{Tables}

1. Thermodynamic Solubility Constants for the Secondary Phase Reaction Network Developed for LAWA44 Glass and Used to Calculate Saturation Indices of Lysimeter Extracts

2. Saturation Indices Calculated for Noteworthy Phases in 1:1 Water Extracts of Background Samples and Outer Top Samples from Lysimeter D-14; Also Included are pH Values of the Water Extracts

3. Saturation Indices Calculated for Noteworthy Phases in 1:1 Water Extracts of Center Samples from Lysimeters D-10 and D-11; Also Included are pH Values of the Extracts

4. Saturation Indices Calculated for Noteworthy Phases in 1:1 Water Extracts of Center Samples from Lysimeter D-14; Also Included are $\mathrm{pH}$ Values of the Extracts 


\subsection{Introduction}

Performance assessment calculations for immobilized low-activity waste (ILAW) glass to be disposed at the Hanford Site depend on simulations of long-term glass corrosion behavior and contaminant transport that are being performed via reactive chemical transport modeling (e.g., STOMP simulations). Confidence in the underlying physical and geochemical processes that are included in conceptual models and are being simulated by computer codes can be significantly enhanced through the use of carefully controlled field testing. Field-testing allows the Integrated Disposal Facility performance assessment (IDF-PA) program to obtain independent and site-relevant data on glass corrosion at a length scale more relevant to the actual disposal system. As a result, these field test data can be used to validate the models used to forecast the long-term behavior of the glass waste form and the resulting environmental impacts.

Although lysimeters have several disadvantages, they are the logical choice for field-testing glass corrosion and contaminant transport for IDF-PA model validation. The lysimeter system provides an approach to combine the low-activity waste (LAW) glass, Hanford soil, and other engineered materials in a well-controlled test at a length-scale that is not practical in the laboratory. The walls of the lysimeter: 1) form a physical boundary in the system being studied that defines a fixed volume for calculating water storage and tracer mass balance, and 2) restrict the geometry of flow within the lysimeter to essentially one dimension. Interpretation of complex temporal variations in tracer release and transport is much simpler in one spatial dimension. The field-scale affords the opportunity to monitor contaminant release and transport in time and space that is not possible in laboratory experiments.

In 2002 and 2003 a set of lysimeter field studies was initiated to field-test glass corrosion and contaminant transport for IDF-PA model validation (Meyer et al. 2001; McGrail et al. 2003). The lysimeter field studies were conducted in existing lysimeters at the Field Lysimeter Test Facility (FLTF) at Hanford. In fiscal year 2010 (FY 2010) after approximately eight years of testing, all three of the lysimeters were excavated and soil samples and glass cylinders retrieved. Portions of the solids from all three lysimeters were archived for potential future solids and solution characterization. In FY 2013 experimental data were collected to determine the elemental concentration profiles of samples obtained from lysimeters to determine the flux of elements from the glass samples as a function of depth. This was accomplished with 1:1 water extracts on sediment samples collected from sediment cores taken from the lysimeters (Pierce et al. 2013). Mineralogical and surface analysis of a select number of glass samples collected from the lysimeter facility was also completed. This work included X-ray diffraction (XRD) and scanning electron microscopy (SEM) analyses. In FY 2014 geochemical modeling of the water extracts was conducted to assess the applicability of the currently used suite of secondary phases. This work consisted of conducting saturation index (SI) calculations on the 1:1 water extracts of sediment samples collected from sediment cores taken from the lysimeters. These results are reported here along with a discussion regarding the implications of the results.

\subsection{Quality Assurance}

This work was conducted with funding from Washington River Protection Solutions (WRPS) under contract 36437-161, ILAW Glass Testing for Disposal at IDF. The work was conducted as part of Pacific Northwest National Laboratory (PNNL) Project 66309, ILAW Glass Testing for Disposal at IDF. 
All research and development (R\&D) work at PNNL is performed in accordance with PNNL's Laboratory-level Quality Management Program, which is based on a graded application of NQA-1-2000, Quality Assurance Requirements for Nuclear Facility Applications, to R\&D activities. To ensure that all client quality assurance (QA) expectations were addressed, the QA controls of the WRPS Waste Form Testing Program (WWFTP) QA program were also implemented for this work. The WWFTP QA program consists of the WWFTP Quality Assurance Plan (QA-WWFTP-001) and associated QA-NSLW-numbered procedures that provide detailed instructions for implementing NQA-1 requirements for $\mathrm{R} \& \mathrm{D}$ work.

The work described in this report was assigned the technology level "Applied Research" and was planned, performed, documented, and reported in accordance with Procedure QA-NSLW-1102, Scientific Investigation for Applied Research. All staff members contributing to the work received proper technical and quality assurance training prior to performing quality-affecting work. 


\subsection{Background and Methods}

\subsection{ILAW Glass Lysimeter Experiments}

The ILAW glass lysimeter field studies experiments were conducted in existing lysimeters at the Field Lysimeter Test Facility (FLTF) at Hanford. The FLTF consists of 14 non-weighing lysimeters and four weighing lysimeters installed within a subsurface concrete vault. The size of the lysimeters was 2-m diameter by 3-m deep. Power and water are available at the site. Use of the existing FLTF infrastructure reduced the overall cost of the ILAW glass corrosion field experiments, while satisfying the scientific requirements of the study. Figure 1 shows the surface of the FLTF along with a schematic of how the glass cylinders were arranged in each lysimeter. Three FLTF lysimeters, designated as D-10, D-11, and D-14, were selected and modified for these experiments.
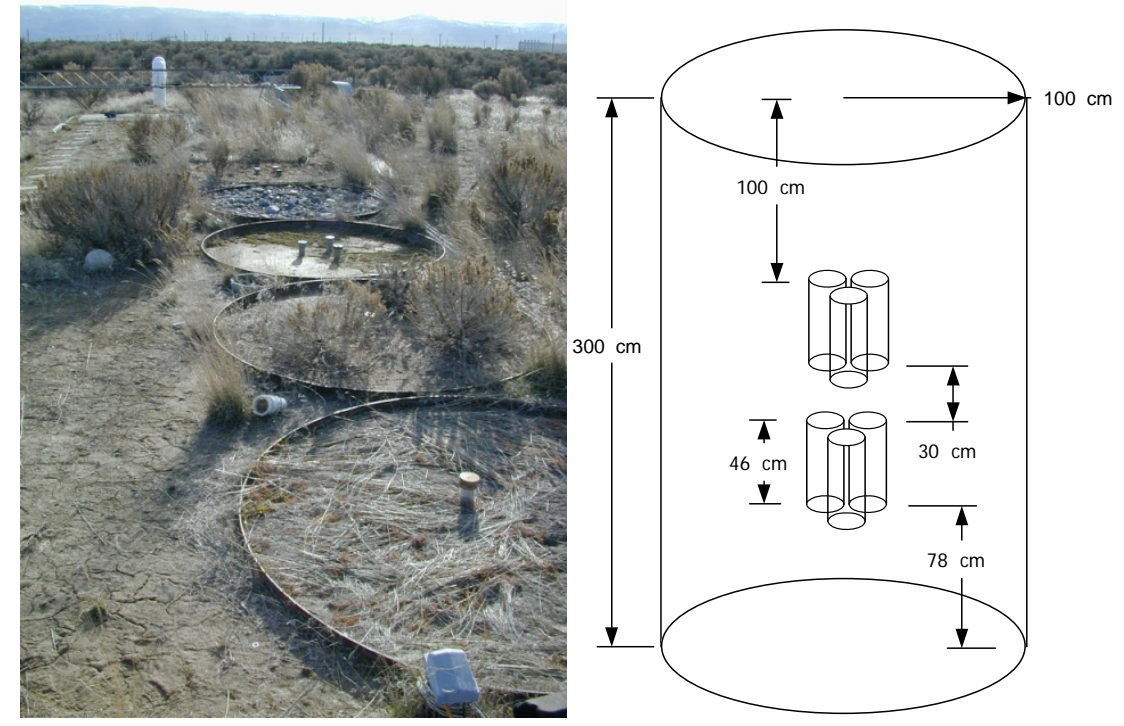

Figure 1. Surface of Field Lysimeter Test Facility (left) Showing Several Lysimeters and a Side View Diagram of ILAW Glass Test Lysimeters Showing that Each Consisted of Two Groups of Three Glass Cylinders at Upper and Lower Levels

The actual glass waste packages to be produced for the IDF will be $213-\mathrm{cm}$-high cylinders, $122-\mathrm{cm}$ in diameter; however, because of the cost to produce and handle full-size glass cylinders, smaller samples (46 cm high and $20 \mathrm{~cm}$ in diameter) were used in the field experiments. These glass samples' dimensions represent a balance between the cost to produce the glass and the desire to represent the key aspects of the flow and transport characteristics of the full-scale facility. Innovatech Services, Inc. prepared twelve HAN28F glass cylinders and six LAWA44 (or equivalent) glass cylinders in 2002 and 2003, respectively (Meyer et al. 2001; McGrail et al. 2003. The glasses were prepared in graphite crucibles, but were transferred to the field in polyvinyl chloride (PVC) containers, which were removed as the glass was buried. The glasses did not include the steel canister of the planned ILAW waste form. A photograph (Figure 2) taken prior to glass burial, illustrates the degree of glass fracturing, which extended throughout the volume of the glass. The fracturing increased the glass surface area and made handling and burial more difficult. 


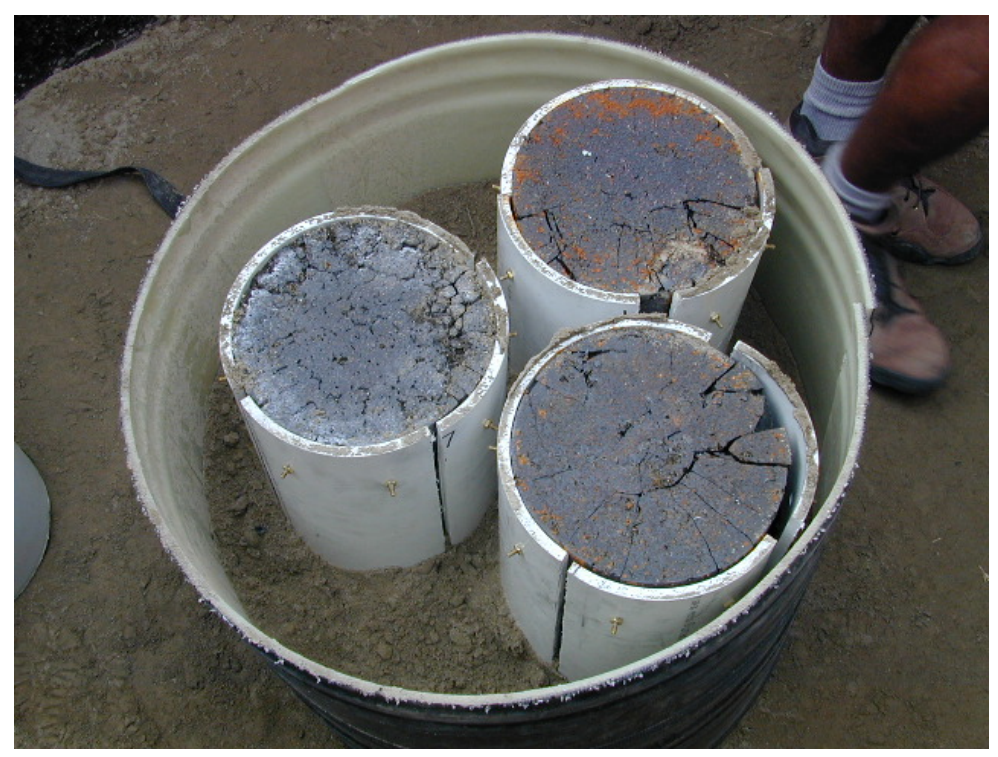

Figure 2. Glass Cylinders Being Buried in a Lysimeter at the Hanford Site

The lysimeter experiments were conducted on the irrigated side of the FLTF; therefore, the experiments received untreated Columbia River water in addition to natural precipitation. The track-mounted overhead irrigation system was set to increase the applied water to three times the average natural precipitation for each month. Irrigation water was typically applied at a rate of $4-\mathrm{mm} / \mathrm{hr}$ in increments ranging from 3 to 344-mm per application (Fayer et al. 1999). The lysimeters were watered at $36 \mathrm{~cm} /$ year in addition to natural precipitation.

At the time this experiment was set up (FY 2002 and FY 2003), it was expected that the glass waste packages would be placed in the IDF using a hexagonal packing arrangement. The hexagonal packing produces two distinct regions within a layer of the glass cylinders: 1) a central region formed by three adjacent cylinders, and 2) the region along the edge of the layer (or row). Because of the differences in the hydraulic properties of the glass and backfill material, it is anticipated that more water will flow between the glass cylinders than through them. As a consequence, the water content in the region between the cylinders is expected to be higher than the water content outside the row(s) of waste packages. Results from Bacon and McGrail (2001) suggest that the chemistry and transport will also be different in these two regions. To represent both regions in the experimental facility, three glass cylinders were arranged as an equilateral triangle, as shown in Figure 1. The glass cylinders were buried in typical Hanford sandy loam or sandy gravel soils in sets of three per level, two levels in each lysimeter, at depths of 1.0 and 1.76 meters to the tops of the logs. Glass weathering and leachate transport simulation results also suggested that the multiple layers of waste packages will affect the chemistry and transport within the IDF. To investigate these effects in this field experiment, two layers of three glass cylinders are included in the experimental design.

\subsection{Lysimeter Sampling and Characterization}

After approximately eight years of testing, all three of the lysimeters were excavated and soil and glass cylinders retrieved in FY 2010. The glass samples in one lysimeter (D-14 with LAWA44 glass) were largely intact when dismantled, as compared with the glass samples in lysimeters D-10 and D-11 (HAN28F glass), which were only found in fragments. Portions of the solids from all three lysimeters 
were archived for potential future solids and solution characterization. The soil and weathered glass samples were stored in refrigerated storage in the Research Technology Laboratory (RTL) Building at PNNL, and later moved to similar storage in the 331 Building.

During excavation and sampling, large-diameter sediment cores were taken approximately $30 \mathrm{~cm}$ from the glass cylinders and small-diameter $(19 \mathrm{~mm})$ cores were collected in the volume between the cylinders approximately $1 \mathrm{~cm}$ from the glass. These soil samples were collected both for the upper and lower glass cylinder arrays (see Figure 3, Figure 4, Figure 5, and Figure 6). Detailed schematics of the locations, diameters, and lengths of the soil cores obtained from the three lysimeters are found in Figure 7, Figure 8, and Figure 9 for lysimeters D-10 and D-11 with HAN28F glass, and D-14 with LAWA44 glass.

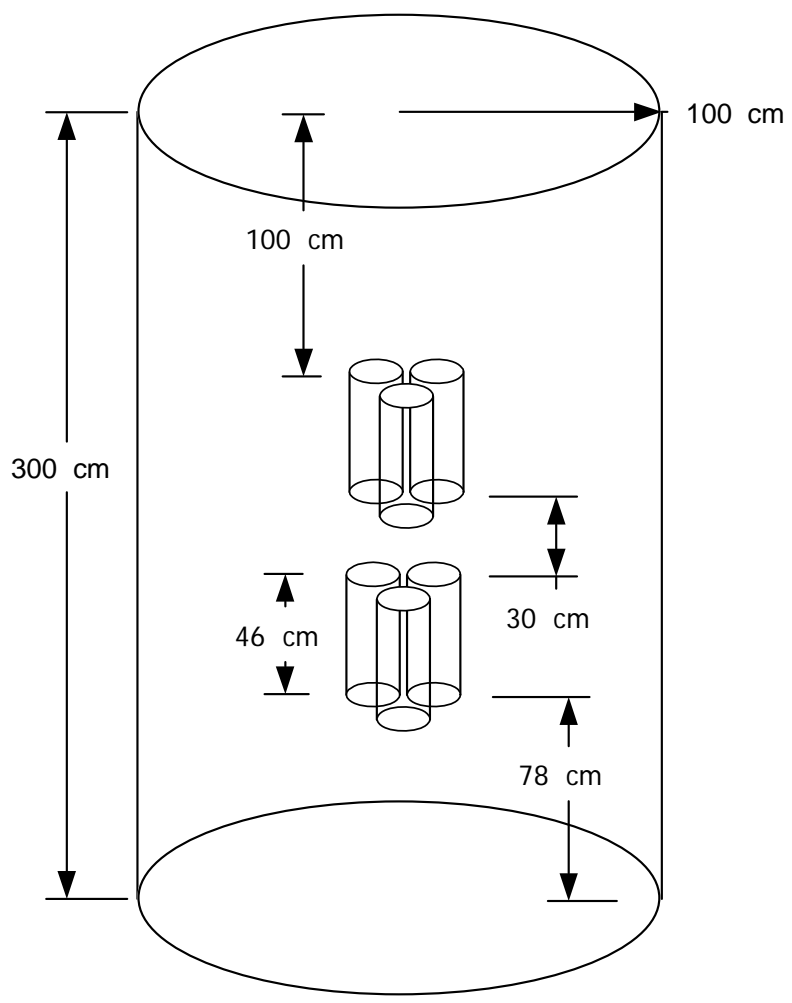

Figure 3. Side View Diagram of ILAW Glass Test Lysimeters Showing that Each Consisted of Two Groups of Three Glass Cylinders at Upper and Lower Levels

For each of the lysimeters, background samples were collected from above the locations of the glass cylinders (indicated as BG in Figure 7, Figure 8, and Figure 9). After the soil cores were brought to the laboratory, 1:1 deionized water extractions were performed. The resulting extracts were analyzed for major and minor cations, anions, $\mathrm{pH}$, and alkalinity. Details of the sample preparation and analysis are available in Pierce et al. (2013). The analytical results of the 1:1 water extracts are also compiled in Pierce et al. (2013).

Selected pieces of glass with sediment attached were subsampled for solid-phase characterization by SEM and XRD. The SEM samples were prepared by first impregnating the sample with epoxy, followed 
by cutting with a diamond saw, polishing, and mounting. Samples for XRD analysis were prepared by scraping off sediment attached to the weathered glass samples; the sediment was then powdered and mounted on glass plates.

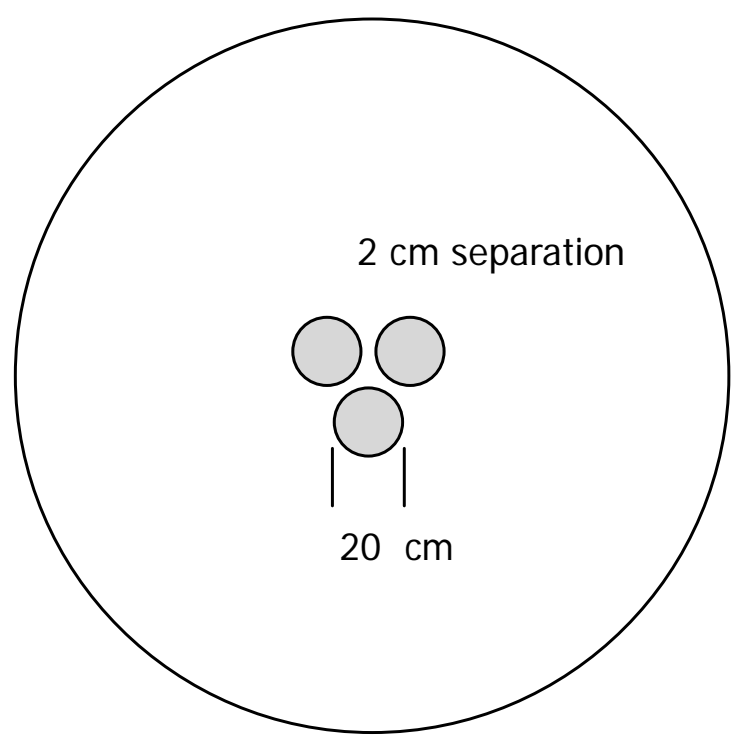

Figure 4. Plan View of Glass Cylinder Arrays in Lysimeters. Sediment samples were collected in two types: large-diameter cores ca. 20-30 cm away from outside of the cylinder array and small-diameter cores between cylinders. 


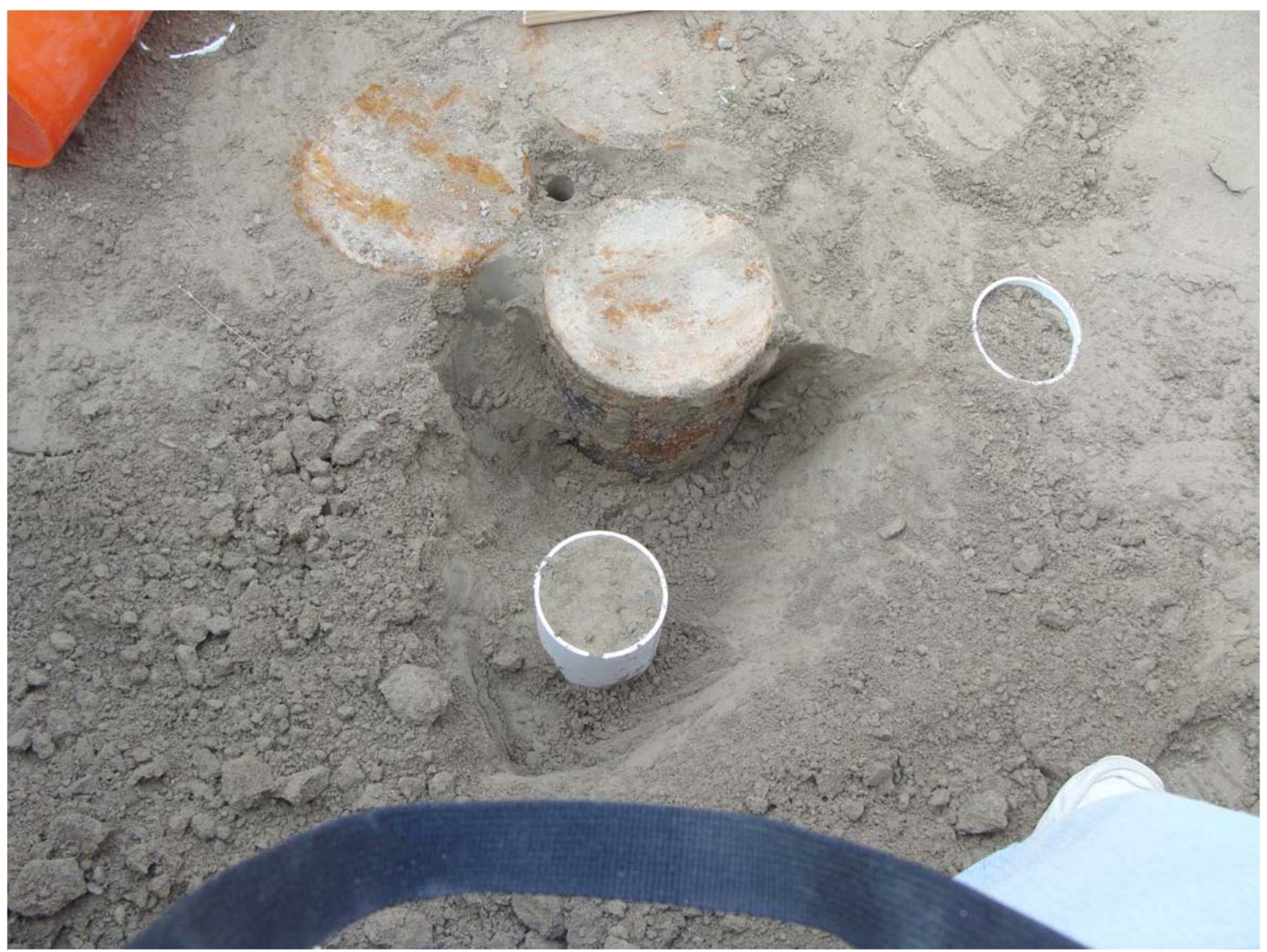

Figure 5. Field Photo of Lysimeter D-14 Showing Locations of Large-Diameter Cores Outside Cylinder Array and the Channel (open hole) of a Small-Diameter Core between Cylinders 


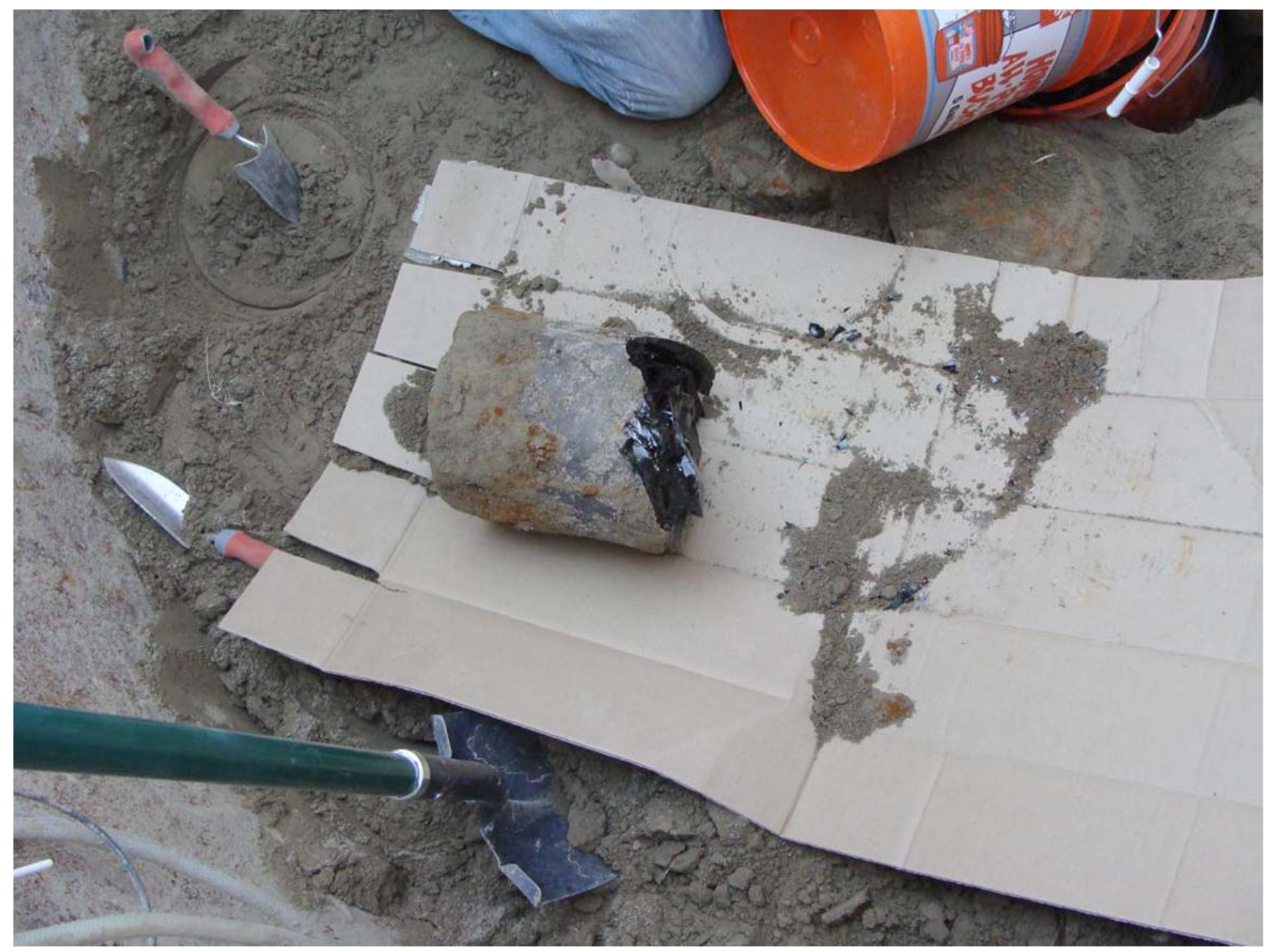

Figure 6. Photograph of Part of a Glass Cylinder (LAWA44) from Lysimeter D-14 Showing the Process of Collection of Glass with Attached Sediment

\subsection{Geochemical Modeling}

Results of the 1:1 water extracts compositions, along with methods used to conduct the extractions, are reported in Piece et al. (2013). Thermodynamic equilibrium modeling was used to calculate mineral saturation indices and to identify solid phases potentially in equilibrium with the 1:1 water extract compositions. For modeling purposes, the redox status of the water extracts was assumed to be approximately in equilibrium with air ( $\mathrm{an}_{2}$ concentration of $8 \mathrm{mg} / \mathrm{L}$ was used in the input). The saturation index is defined as $\mathrm{SI}=\log \left(\mathrm{Q} / \mathrm{K}_{\mathrm{sp}}\right)$, where $\mathrm{Q}$ is the activity product and $\mathrm{K}_{\mathrm{sp}}$ is the mineral solubility product at equilibrium at the temperature of interest. Minerals with SI values near zero (within $\pm \sim 0.5$ ) are generally considered to be at or near equilibrium, more positive values are considered oversaturated, and more negative values are considered undersaturated with respect to the solution composition. Geochemist's Workbench ${ }^{\circledR}$ version 8.09 (Bethke and Yeakel 2010) was used to calculate the mineral SIs for the leachates. The thermodynamic database "thermo.com.V8.R6+.dat" was used for the modeling calculations. Some of the data in the thermodynamic database were modified to better represent the solubility of expected secondary phases (Pierce et al. 2004). Table 1 shows the thermodynamic solubility constants used to model secondary phase formation in the reaction network developed for LAWA44 glass (Pierce et al. 2004). This set of secondary phases was confirmed to be 
largely representative of the secondary phases that form during product consistency tests (PCTs) performed with a large number of glass compositions (Pierce et al. 2013).

\section{Lysimeter D-10}

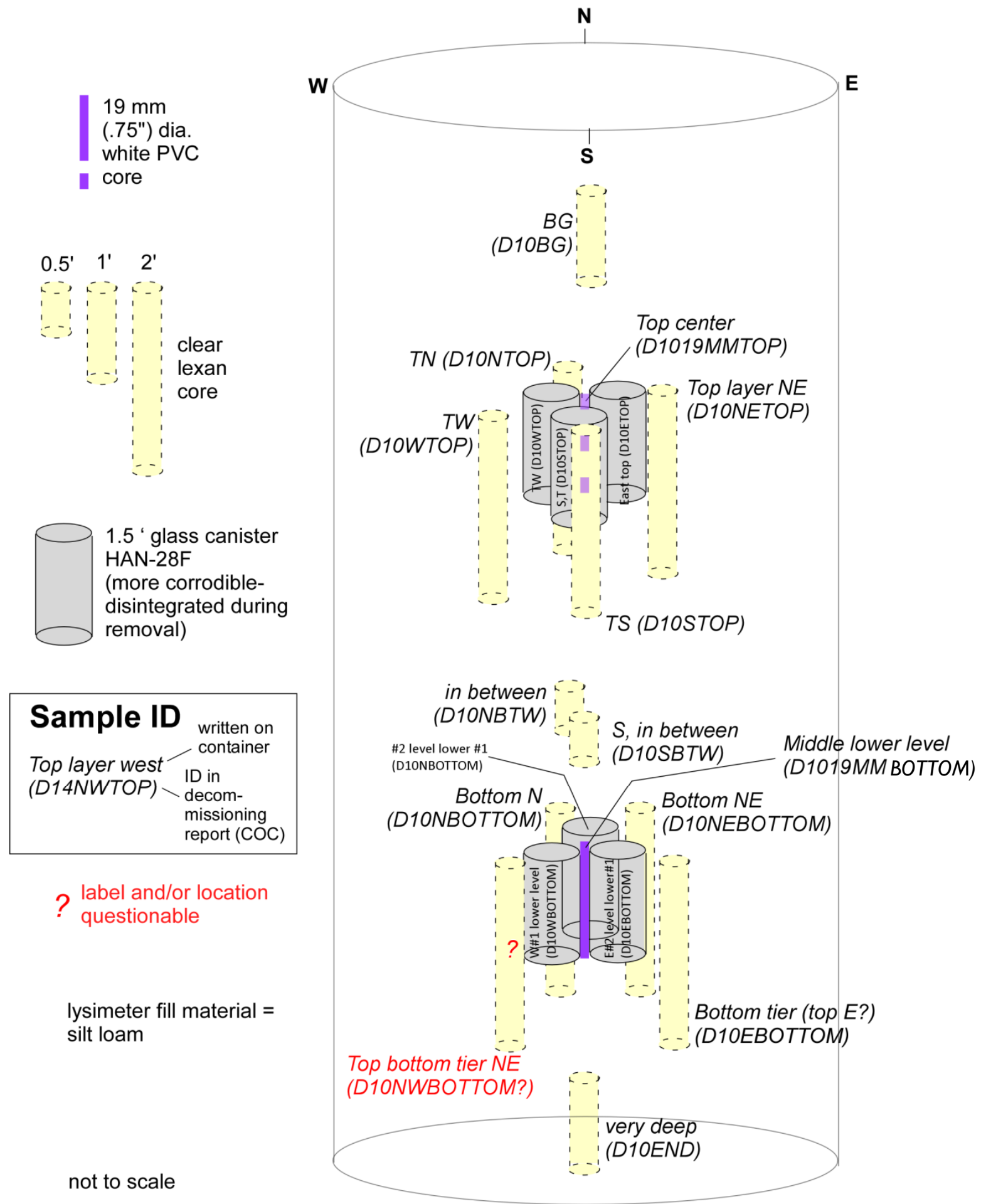

Figure 7. Lysimeter D-10 Glass and Sediment Core Locations 


\section{Lysimeter D-11}

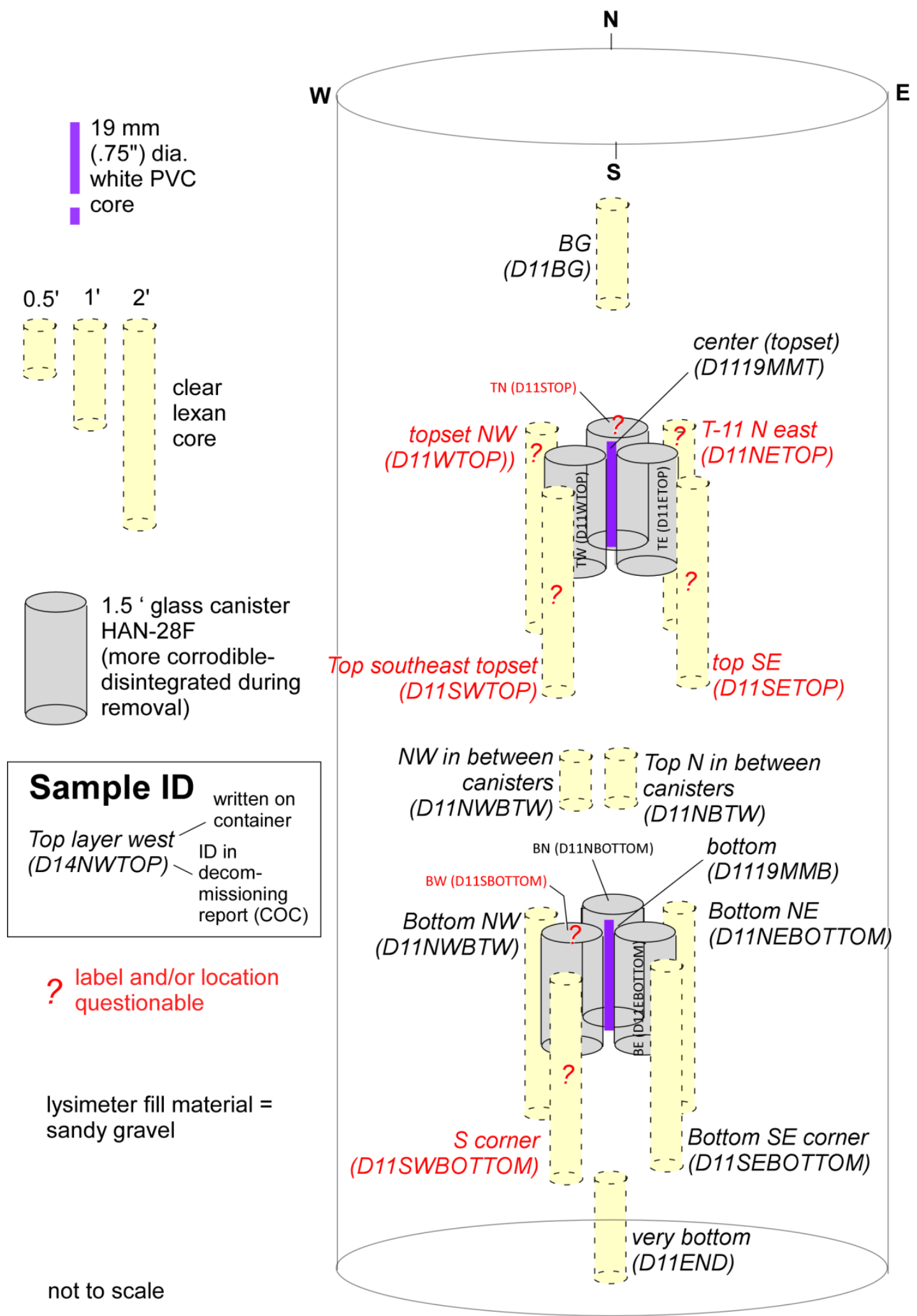

Figure 8. Lysimeter D-11 Glass and Sediment Core Locations 


\section{Lysimeter D-14}

$19 \mathrm{~mm}$ (.75") dia. white PVC core

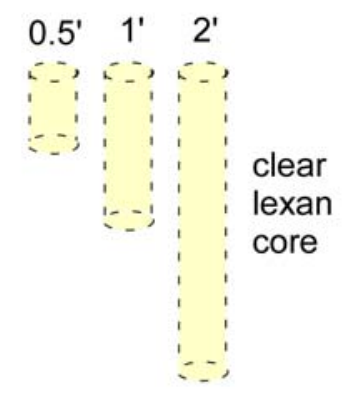

1.5 ' glass canister LAWA44 (portions of canister intact)

\section{Sample ID \\ Top layer west ID in $(D 14 N W T O P) \backslash$ decom- missioning report (COC)}

? label and/or location questionable

lysimeter fill material = silt loam

not to scale

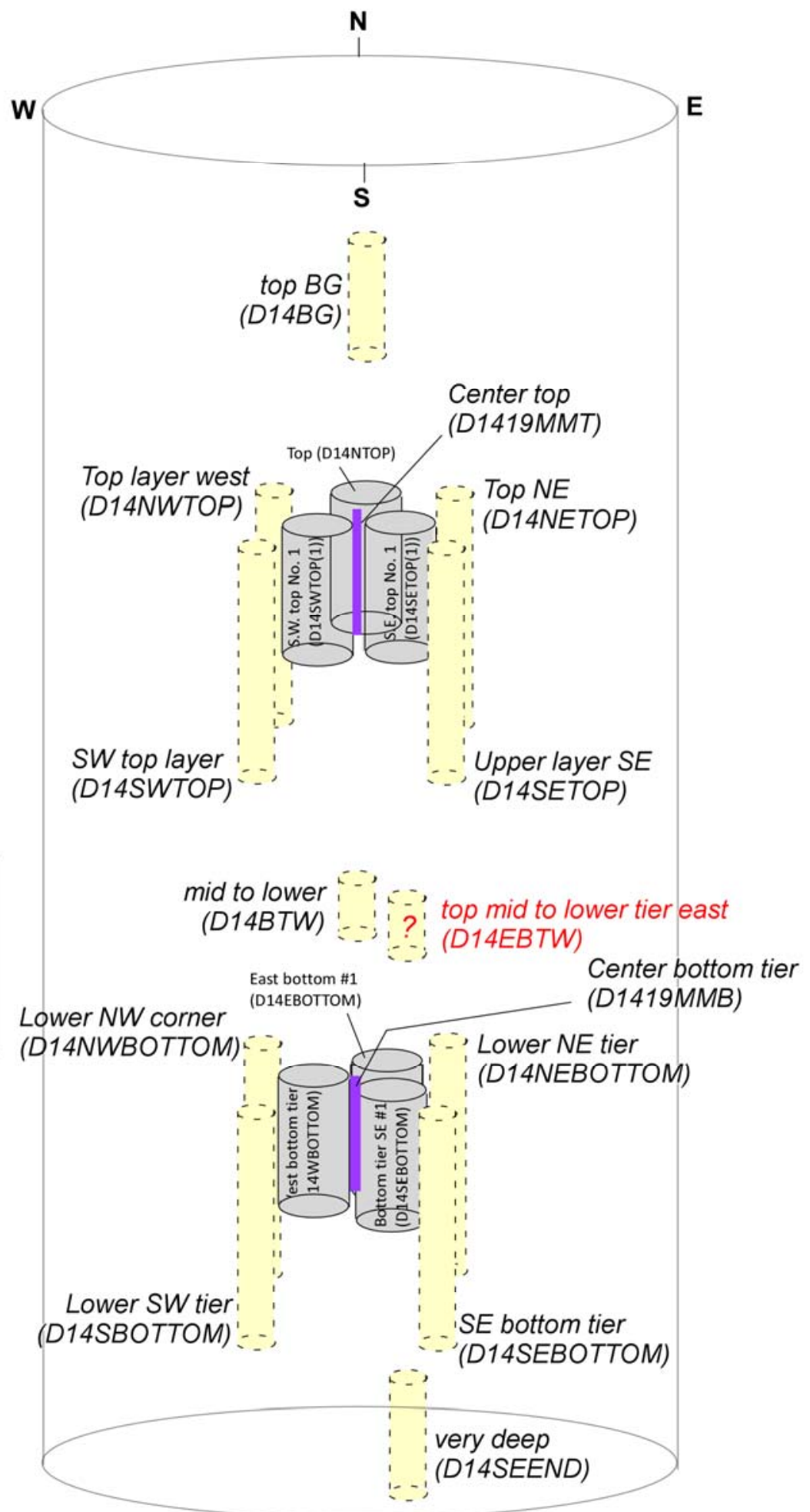

Figure 9. Lysimeter D-14 Glass and Sediment Core Locations 
Table 1. Thermodynamic Solubility Constants for the Secondary Phase Reaction Network Developed for LAWA44 Glass and Used to Calculate Saturation Indices of Lysimeter Extracts (Pierce et al. 2004)

\begin{tabular}{lcc}
\hline \multicolumn{1}{c}{ Phase } & Reaction & $\begin{array}{c}\text { Log K } \\
\left(20^{\circ} \mathrm{C}\right)\end{array}$ \\
\hline Analcime $\left(\mathrm{Na}_{0.96} \mathrm{Al}_{0.96} \mathrm{Si}_{2.04} \mathrm{O}_{6} \cdot \mathrm{H}_{2} \mathrm{O}\right)$ & analcime $+3.84 \mathrm{H}^{+} \leftrightarrow 0.96 \mathrm{Al}^{3+}+0.96 \mathrm{Na}^{+}+$ & 9.34 \\
$\mathrm{TiO}_{2}(\mathrm{am})$ & $2.04 \mathrm{SiO}_{2}(\mathrm{aq})+2.92 \mathrm{H}_{2} \mathrm{O}$ & -6.56 \\
$\mathrm{ZrO}_{2}(\mathrm{am})$ & $\mathrm{TiO}_{2}+2 \mathrm{H}_{2} \mathrm{O} \leftrightarrow \mathrm{Ti}(\mathrm{OH})_{4}(\mathrm{aq})$ & -5.47 \\
Calcite $\left(\mathrm{CaCO}_{3}\right)$ & $\mathrm{ZrO}_{2}+2 \mathrm{H}^{+} \leftrightarrow \mathrm{Zr}(\mathrm{OH})_{2}{ }^{2+}$ & 1.92 \\
Chalcedony $\left(\mathrm{SiO}_{2}\right)$ & $\mathrm{CaCO}_{3}+\mathrm{H}^{+} \leftrightarrow \mathrm{Ca}^{2+}+\mathrm{HCO}_{3}{ }^{-}$ & -3.55 \\
$\mathrm{Fe}(\mathrm{OH})_{3}(\mathrm{~s})$ & $\mathrm{SiO}_{2} \leftrightarrow \mathrm{SiO}_{2}(\mathrm{aq})$ & 9.42 \\
$\mathrm{Gibbsite}\left[\mathrm{Al}(\mathrm{OH})_{3}\right]$ & $\mathrm{Fe}(\mathrm{OH})_{3}(\mathrm{am})+3 \mathrm{H}^{+} \leftrightarrow \mathrm{Fe}^{3+}+3 \mathrm{H}_{2} \mathrm{O}$ & 8.08 \\
Sepiolite $\left[\mathrm{Mg}_{4} \mathrm{Si}_{6} \mathrm{O}_{15}(\mathrm{OH})_{2} \cdot 6 \mathrm{H}_{2} \mathrm{O}\right]$ & $\mathrm{Al}(\mathrm{OH})_{3}+3 \mathrm{H}^{+} \leftrightarrow \mathrm{Al}^{3+}+3 \mathrm{H}_{2} \mathrm{O}$ & 45.83 \\
$\mathrm{Zn}(\mathrm{OH})_{2}-\gamma$ & sepiolite $+8 \mathrm{H}^{+} \leftrightarrow 4 \mathrm{Mg}^{2+}+6 \mathrm{SiO}_{2}(\mathrm{aq})+11 \mathrm{H}_{2} \mathrm{O}$ & 11.88 \\
\hline
\end{tabular}




\subsection{Modeling Results and Discussion}

Saturation indices were calculated for 43 1:1 water extract samples. The discussion in this section is focused primarily on phases that are part of the secondary phase reaction network established for LAWA44 glass (Pierce et al. 2004). This secondary phase reaction network is based on modeling of LAWA44 PCT results and was later found to be largely consistent with modeling of PCT results conducted on a large number of other ILAW glass samples (Pierce et al. 2013). Other plausible secondary phases that warrant consideration for possible inclusion in or replacement of other phases within the secondary phase reaction network are also included in the discussion. Phases with saturation indices that are near equilibrium but are likely to be present naturally in the background sediments (e.g., dolomite and coesite) are not discussed.

\subsection{Saturation Indices of 1:1 Water Extracts}

Saturation indices calculated for the most relevant phases in 1:1 water extracts of selected samples are shown in Table 2, Table 3, and Table 4. Saturation index results for $\mathrm{TiO}_{2}(\mathrm{am}), \mathrm{ZrO}_{2}(\mathrm{am})[(\mathrm{am})$ indicates an amorphous phase], and sepiolite (Table 1) were not included in the tables because Ti and $\mathrm{Zr}$ were not detected above the estimated quantification limit (EQL) in any of the samples and sepiolite was always highly undersaturated. Also included in the tables are the measured $\mathrm{pH}$ values of the water extracts. Elevated $\mathrm{pH}$ relative to background is a good indicator of an influence from glass dissolution on the sediments. Results for background samples and outer top samples from lysimeter D-14 are shown in Table 2. The outer top samples from lysimeter D-14 are included in Table 2 because these samples do not appear to have been significantly impacted by the glass corrosion and are therefore considered to be representative of background samples. Results for the center samples from lysimeters D-10 and D-11 (HAN28F glass) are shown in Table 3 and center samples from lysimeter D-14 (LAWA44 glass) are shown in Table 4. Besides the background samples, the discussion is focused on the center samples because these samples were located in closest proximity to the glass samples and have the highest potential for impacts from glass dissolution.

Review of the data in Table 2 indicates that for all the background samples, calcite $\left(\mathrm{CaCO}_{3}\right)$ and chalcedony $\left(\mathrm{SiO}_{2}\right)$ are near-equilibrium in all samples. With the exception of sample D10BG, the aluminum concentrations in all samples were below the EQL. For D10BG, gibbsite was highly oversaturated, but dawsonite appears to be essentially at equilibrium. Iron was below the EQL in all the background samples, so SI values could not be calculated for $\mathrm{Fe}(\mathrm{OH})_{3}(\mathrm{am})$ [Table 2]. In the absence of sepiolite, several alternative phases were considered for controlling magnesium concentrations. These are magnesite $\left(\mathrm{MgCO}_{3}\right)$, chlinochlore-7 $\mathrm{A}\left[\mathrm{Mg}_{5} \mathrm{Al}_{2} \mathrm{Si}_{3} \mathrm{O}_{10}(\mathrm{OH})_{8}\right]$, and saponite- $\mathrm{Mg}$ $\left[\mathrm{Mg}_{3.165} \mathrm{Al}_{0.33} \mathrm{Si}_{3.67} \mathrm{O}_{10}(\mathrm{OH})_{2}\right]$. Magnesite was consistently well below equilibrium in all the background samples. SI values for chlinochlore-7A and saponite-Mg could be calculated only for sample D10BG because aluminum was below the EQL for all other samples. For sample D10BG, chlinochlore-7A was highly undersaturated and saponite- $\mathrm{Mg}$ was oversaturated. The three zinc-containing phases $\left[\mathrm{Zn}(\mathrm{OH})_{2}-\gamma\right.$, zincite $(\mathrm{ZnO})$, and smithsonite $\left.\left(\mathrm{ZnCO}_{3}\right)\right]$ were all unsaturated in the background samples. The $\mathrm{pH}$ of the background sample extracts ranged from 7.88 to 8.11 , with an average of 8.04. 
Table 2. Saturation Indices Calculated for Noteworthy Phases in 1:1 Water Extracts of Background Samples and Outer Top Samples from Lysimeter D-14; Also Included are pH Values of the Water Extracts (blank cells indicate that a phase component was below the EQL, precluding calculation of SI values)

\begin{tabular}{lccccccc}
\hline & \multicolumn{7}{c}{ Sample ID } \\
\cline { 2 - 8 } \multicolumn{1}{c}{ Phase } & D10BG & D11BG & D14BG & D14SWTOP & D14NWTOP & D14NETOP & D14SETOP \\
\hline Analcime & -1.74 & - & - & - & - & - & - \\
Calcite & -0.55 & -0.37 & -0.36 & -0.25 & -0.33 & -0.32 & -0.30 \\
Chalcedony & 0.18 & 0.20 & 0.27 & 0.35 & 0.33 & 0.32 & 0.32 \\
Fe(OH) $)_{3}(\mathrm{am})$ & - & - & - & - & - & - & - \\
Gibbsite & 2.40 & - & - & - & - & - & - \\
Dawsonite & -0.27 & - & - & - & - & - & - \\
Magnesite & -1.62 & -1.37 & -1.26 & -1.26 & -1.27 & -1.26 & -1.19 \\
Clinochlore-7A & -3.86 & - & - & - & - & - & - \\
Saponite-Mg & 0.85 & - & - & - & - & - & - \\
Zn $(\mathrm{OH})_{2}-\gamma$ & -1.70 & -1.45 & - & -1.37 & - & - & -1.34 \\
Zincite & -1.30 & -1.04 & - & -0.96 & - & - & -0.93 \\
Smithsonite & -1.12 & -1.02 & - & -0.92 & - & - & -0.93 \\
pH & 7.88 & 8.04 & 8.02 & 8.09 & 8.08 & 8.07 & 8.11 \\
\hline
\end{tabular}

The center samples collected from the D-10 and D-11 lysimeters are expected to have the highest influence from the glass samples. This is because they are located within the center of the three glass samples (Figure 7 and Figure 8) and these two lysimeters contained HAN28F glass. This glass corrodes relatively quickly (Meyer et al. 2001) and will likely impact adjacent sediments to a greater extent than the LAWA44 glass in the D-14 lysimeter. This is apparent from the $\mathrm{pH}$ measurements (Table 3), which range from 8.18 to 9.97 with an average of 9.02 . These values clearly indicate that corrosion of the HAN28F glass has measurably impacted the sediments located near the center.

The SI values shown in Table 3 indicate that analcime is essentially at equilibrium in the majority of the samples (5 out of 7). With the exception of sample D1019MMB_ts, gibbsite is significantly oversaturated in all samples. Dawsonite is essentially at saturation for four of the seven samples (D1019MMTOP, D1019MMB_ts, D1019MMB_bs, and D1119MMT_bs) and somewhat oversaturated in three of the samples (D1119MMT_ts, D1119MMB_ts, and D1119MMB_bs). These results indicate that dawsonite may be a more appropriate phase in the secondary phase reaction network than gibbsite under field conditions. This could be due to the presence of a significant pool of calcite in the sediments that provides a continuous source of carbonate, and is in contrast to the PCT tests were carbonate concentrations are limited. At this time the presence of dawsonite at the surface of corroding glass has not been confirmed. 
Table 3. Saturation Indices Calculated for Noteworthy Phases in 1:1 Water Extracts of Center Samples from Lysimeters D-10 and D-11 (HAN28F glass); Also Included are pH Values of the Extracts (blank cells indicate that a phase component was below the EQL, precluding calculation of SI values)

\begin{tabular}{lccccccc}
\hline & \multicolumn{7}{c}{ Sample ID } \\
\cline { 2 - 7 } \multicolumn{1}{c}{ Phase } & $\begin{array}{c}\text { D1019 } \\
\text { MMTOP }\end{array}$ & $\begin{array}{c}\text { D1019 } \\
\text { MMB_ts }\end{array}$ & $\begin{array}{c}\text { D1019 } \\
\text { MMB_bs }\end{array}$ & $\begin{array}{c}\text { D1119 } \\
\text { MMT_ts }\end{array}$ & $\begin{array}{c}\text { D1119 } \\
\text { MMT_bs }\end{array}$ & $\begin{array}{c}\text { D1119 } \\
\text { MMB_ts }\end{array}$ & $\begin{array}{c}\text { D1119 } \\
\text { MMB_bs }\end{array}$ \\
\hline Analcime & -1.52 & -0.22 & -1.22 & 0.05 & -0.08 & -0.29 & -0.22 \\
Calcite & -0.45 & 1.44 & 0.98 & 1.09 & 1.16 & 0.11 & 0.18 \\
Chalcedony & 0.06 & 0.11 & -0.18 & 0.34 & 0.26 & 0.35 & 0.36 \\
Fe(OH $)_{3}(\mathrm{am})$ & -2.54 & -2.07 & - & -2.04 & -2.10 & -2.02 & -2.00 \\
Gibbsite & 2.25 & 1.09 & 0.15 & 1.92 & 1.49 & 2.61 & 2.54 \\
Dawsonite & -0.03 & 0.04 & -0.25 & 0.71 & 0.37 & 0.70 & 0.63 \\
Magnesite & -1.88 & 0.11 & 0.10 & -0.52 & -0.24 & -1.31 & -1.25 \\
Clinochlore-7A & -4.62 & 9.10 & 5.64 & 3.89 & 6.09 & -0.44 & 0.35 \\
Saponite-Mg & 0.30 & 10.15 & 8.33 & 6.48 & 8.13 & 3.11 & 3.70 \\
Zn(OH $)_{2}-\gamma$ & -1.06 & 2.14 & 2.21 & - & - & -0.90 & -0.67 \\
Zincite & -0.66 & 2.54 & 2.62 & - & - & -0.49 & -0.27 \\
Smithsonite & -0.72 & 1.29 & 1.50 & - & - & -0.51 & -0.39 \\
pH & 8.18 & 9.84 & 9.97 & 9.01 & 9.38 & 8.33 & 8.42 \\
\hline
\end{tabular}

Chalcedony is virtually at equilibrium for all samples in Table 3. In contrast, calcite is near equilibrium for only three (D1019MMTOP, D1119MMB_ts, and D1119MMB_bs) of the seven samples. Four of the samples (D1019MMB_ts, D1019MMB_bs, D1119MMT_ts, and D1119MMT_bs) are significantly oversaturated with respect to calcite. This oversaturation with respect to calcite is correlated with $\mathrm{pH}$; all of the extracts with oversaturated SI values have $\mathrm{pH}$ values of 9 or above. As ILAW glasses corrode, $\mathrm{NaOH}$ is released to solution, increasing the $\mathrm{pH}$ and sodium concentration. As the sodium concentration increases, it can exchange for calcium on ion-exchange sites of clay minerals, increasing the calcium concentration in solution. As the $\mathrm{pH}$ in solution increases, bicarbonate is increasingly deprotonated to carbonate. Both of these processes result in an increase in the SI for calcite. The water extractions were shaken for only one hour. Apparently this was insufficient time for calcite to precipitate and reestablish equilibrium. It is possible that under field conditions, equilibrium would occur because contact times are much greater than that of the extraction tests.

For samples that had quantifiable concentrations of iron, the SI values for $\mathrm{Fe}(\mathrm{OH})_{3}(\mathrm{am})$ all fell within a relatively narrow range $(-2.13 \pm 0.20)$. This suggests that these samples are at equilibrium with an $\mathrm{Fe}(\mathrm{OH})_{3}(\mathrm{~s})$ phase that has a lower solubility than that assumed for the secondary phase reaction network. This would be consistent with an $\mathrm{Fe}(\mathrm{OH})_{3}(\mathrm{~s})$ phase that is more crystalline than the $\mathrm{Fe}(\mathrm{OH})_{3}(\mathrm{am})$ phase assumed for the secondary phase reaction network, and could have been promoted by repeated wet-dry cycles that would have occurred in the lysimeter tests. This would not occur in the PCT experiments because the glass samples are maintained continuously in water.

In four of the seven samples (D1019MMB_ts, D1019MMB_bs, D1119MMT_ts, and D1119MMT_bs) magnesite is essentially at equilibrium. These samples are the samples that have $\mathrm{pH}$ values of 9 or above. In the other three samples (with $\mathrm{pH}$ values of 8.42 or less) magnesite is significantly undersaturated. In the case of sample D1019MMTOP, saponite-Mg is near saturation and 
chlinochlore-7A is highly undersaturated. For samples D1119MMB_ts and D1119MMB_bs, chlinochlore-7A appears to be near saturation and saponite-Mg is highly oversaturated. It is expected that precipitation of secondary phases will be greatest at the glass-water interface, where $\mathrm{pH}$ and concentrations of soluble constituents from the glass will be highest. Because of this, it is hypothesized that magnesite is the most probable secondary phase for controlling the concentrations of $\mathrm{Mg}$ that leach from glass. At this time the presence of magnesite at the surface of corroding glass has not been confirmed.

Saturation index values calculated for the zinc minerals shown in Table 3 suggest that when there are measurable values of zinc; zincite $(\mathrm{ZnO})$ is closer to being in equilibrium than $\mathrm{Zn}(\mathrm{OH})_{2}-\gamma$ for samples D1010MMTOP, D1119MMB_ts, and D1119MMB_bs. For samples D1019MMB_ts and D1019MMB_bs, all the zinc phases are highly oversaturated, with smithsonite $\left(\mathrm{ZnCO}_{3}\right)$ being the least oversaturated.

Saturation indices calculated for phases of interest in the 1:1 water extracts of center samples from lysimeter D-14 (LAWA44 glass) and pH values of the extracts are shown in Table 4. Based on the $\mathrm{pH}$ values measured for these samples, it is apparent that corrosion of the LAWA44 had a minimal effect on the sediments from the D-14 lysimeter. Both calcite and chalcedony are effectively at equilibrium for all four samples, similar to the background samples. Many of the samples had components that were below their EQLs, precluding the calculation of SI values for a number of phases. With the exception of gibbsite, dawsonite and saponite-Mg for sample D1419MMT_bs, nearly all the SI values that were calculated indicated that the remaining phases were undersaturated. The most significant impacts due to glass corrosion were observed in sample D1419MMT_bs, which had an extract pH of 8.33. In this sample both gibbsite and saponite-Mg were significantly oversaturated. Analcime was undersaturated, but not to a great extent, and dawsonite appeared to be essentially at saturation. All these results are consistent with the observation that the impact on lysimeter D-14 sediments due to corrosion of LAWA44 glass was minimal. 
Table 4. Saturation Indices Calculated for Noteworthy Phases in 1:1 Water Extracts of Center Samples from Lysimeter D-14 (LAWA44 glass); Also Included are pH Values of the Extracts (blank cells indicate that a phase component was below the EQL, precluding calculation of SI values)

\begin{tabular}{lcccc}
\hline & \multicolumn{5}{c}{ Sample ID } \\
\cline { 2 - 5 } \multicolumn{1}{c}{ Phase } & $\begin{array}{c}\text { D1419 } \\
\text { MMT_ts }\end{array}$ & $\begin{array}{c}\text { D1419 } \\
\text { MMT_bs }\end{array}$ & $\begin{array}{c}\text { D1419 } \\
\text { MMB_ts }\end{array}$ & $\begin{array}{c}\text { D1419 } \\
\text { MMB_bs }\end{array}$ \\
\hline Analcime & - & -0.88 & - & - \\
Calcite & -0.33 & 0.25 & -0.26 & -0.18 \\
Chalcedony & 0.26 & 0.33 & 0.31 & 0.27 \\
Fe(OH) $)_{3}(\mathrm{am})$ & - & -2.64 & - & - \\
Gibbsite & - & 2.01 & - & - \\
Dawsonite & - & 0.18 & - & - \\
Magnesite & -1.22 & -1.18 & -1.23 & -1.12 \\
Clinochlore-7A & - & -1.30 & - & - \\
Saponite-Mg & - & 3.09 & - & - \\
Zn(OH) ${ }_{2}-\gamma$ & -1.94 & - & - & -1.55 \\
Zincite & -1.54 & - & - & -1.15 \\
Smithsonite & -1.25 & - & - & -0.97 \\
pH & 7.83 & 8.33 & 7.97 & 8.02 \\
\hline
\end{tabular}




\subsection{Conclusions}

The results of the geochemical modeling of the 1:1 water extracts suggest that the secondary phase reaction network determined for ILAW glass that were derived from PCT results may be different when glass is buried in Hanford sediment. Several factors may be responsible for this effect. The most important geochemical factor is the availability of carbonate minerals (e.g., calcite) that typically occurs in Hanford sediment. The presence of carbonate-containing minerals in the sediments results in pore waters that are typically in equilibrium with calcite. It is believed that this results in dawsonite $\left[\mathrm{NaAl} \mathrm{CO} 3(\mathrm{OH})_{2}\right]$ replacing gibbsite within the secondary phase reaction network when glass in corroded in Hanford sediment. It was also found that sepiolite was not likely to form as a secondary phase when glass corrodes in Hanford sediment. Potential alternative phases include magnesite $\left(\mathrm{MgCO}_{3}\right)$ and clinochlore $\left[\mathrm{Mg}_{5} \mathrm{Al}_{2} \mathrm{Si}_{3} \mathrm{O}_{10}(\mathrm{OH})_{8}\right]$. The repeated wetting and drying cycles that occurred in the lysimeter experiments may have also had an impact on the nature of the secondary phase reaction network. For example, the solubility of several sparingly soluble metal oxides [e.g., $\mathrm{Fe}(\mathrm{OH})_{3}, \mathrm{TiO}_{2}(\mathrm{am})$, and $\left.\mathrm{ZrO}_{2}(\mathrm{am})\right]$ was much lower in the 1:1 extracts of the lysimeter samples than was found for the PCT experiments.

In order to validate that the alternative secondary phases predicted by the geochemical modeling, it is important to conduct solid-phase characterization to demonstrate their occurrence at or near the surface of the glass. Solid-phase characterization work was done as part of the lysimeter sample characterization completed in FY 2013 (Pierce et al. 2013); however, the results were inconclusive with regards to validating the alternative secondary phases identified by the geochemical modeling of the 1:1 water extracts of the lysimeter samples. To overcome this impediment, a complimentary approach is recommended to optimize our ability to identify the secondary phases that form during glass corrosion in contact with Hanford sediments. This will involve a flow-through column experiment designed to optimize secondary phase formation on glass samples that have been fabricated specifically to optimize sample preparation and characterization methods. Advanced microscopy techniques including SEM-EDS (scanning electron microscopy-energy dispersive spectroscopy), FIB (focused ion beam), and TEM-SAED (transmission electron microscopy-selected area electron diffraction) will be used to characterize the secondary solid phases that form. SEM-EDS can be used to identify likely secondary phases based upon morphology and elemental composition. Once likely secondary phases have been identified by SEM-EDS, phase confirmation can be made using TEM-SAED. The FIB can be used to isolate submicron-size subsamples found using SEM-EDS for TEM analysis (TEM requires very thin samples, typically $\sim 100$ nanometers). Once the sample prepared with the FIB has been transferred to the TEM, SAED analysis can be used to identify the specific phase under interrogation. 


\subsection{References}

Bacon DH and BP McGrail. 2001. Waste Form Release Calculations for the 2001 Immobilized

Low-Activity Waste Performance Assessment. PNNL-13369, Pacific Northwest National Laboratory, Richland, Washington.

Bethke C. and S Yeakel. 2010. The Geochemist's Workbench, Release 8.0, Reference Manual. Urbana, Illinois: Hydrogeology Program.

Fayer MJ, EM Murphy, JL Downs, FO Khan, CW Lindenmeier, and BN Bjornstad. 1999. Recharge Data Package for the Immobilized Low-Activity Waste 2001 Performance Assessment. PNNL-13033, Pacific Northwest National Laboratory, Richland, Washington.

McGrail B, D Bacon, R Serne, and E Pierce. 2003. A Strategy to Assess Performance of Selected Low-Activity Waste Forms in an Integrated Disposal Facility. PNNL-14362, Pacific Northwest National Laboratory, Richland, Washington.

Meyer PD, BP McGrail, and DH Bacon. 2001. Test Plan for Field Experiments to Support the Immobilized Low-Activity Waste Disposal Performance Assessment at the Hanford Site. PNNL-13670, Pacific Northwest National Laboratory, Richland, Washington.

Pierce EM, BP McGrail, EA Rodriguez, HT Schaef, KP Saripalli, RJ Serne, KM Krupka, PF Martin, SR Baum, KN Geiszler, LR Reed, and WJ Shaw. 2004. Waste Form Release Data Package for the 2005 Integrated Disposal Facility Performance Assessment. PNNL-14805, Pacific Northwest National Laboratory, Richland, Washington.

Pierce EM, SN Kerisit, EJ Krogstad, SD Burton, BN Bjornstad, VL Freedman, KJ Cantrell, MM Valenta, JV Crum, and JH Westsik, Jr. 2013. Integrated Disposal Facility FY 2012 Glass Testing Summary Report. PNNL-21812 Rev. 1, Pacific Northwest National Laboratory, Richland, Washington. 



\section{Appendix A}

\section{Geochemist's Workbench Output}





\section{Appendix A}

\section{Geochemist's Workbench Output}

\section{Sample D10BG}

$\begin{array}{ll}\text { Step \# } \odot & \\ \text { Temperature }=20 . \odot \mathrm{C} \\ \text { pH }=7.880 \\ \text { Eh }=0.7610 \text { volts } \\ \text { Ionic strength } & = \\ \text { Charge imbalance } & = \\ \text { Activity of water } & = \\ \text { Solvent mass } & = \\ \text { Solution mass } & = \\ \text { Solution density } & = \\ \text { Chlorinity } & = \\ \text { Dissolved solids } & = \\ \text { Elect. conductivity } & = \\ \text { Hardness } & = \\ \text { carbonate } & = \\ \text { non-carbonate } & = \\ \text { Rock mass } & = \\ \text { Carbonate alkalinity } & = \\ \text { Water type } & =\end{array}$

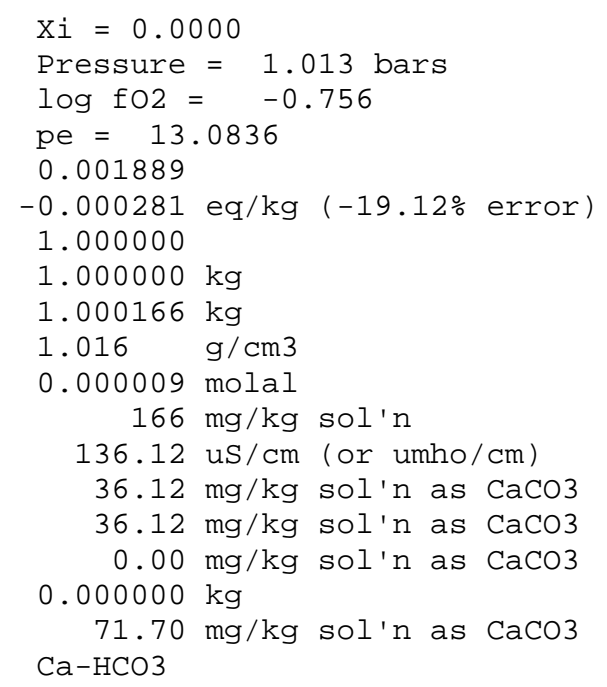

No minerals in system.

\begin{tabular}{|c|c|c|c|c|}
\hline Aqueous species & molality & $\mathrm{mg} / \mathrm{kg}$ sol'n & act. coef. & $\log$ act. \\
\hline & - - - - - - - & & & \\
\hline HCO3 - & ๑ . 001415 & 86.33 & $\odot .9534$ & -2.8700 \\
\hline $\mathrm{Na}+$ & ๑. .0๑९5382 & 12.37 & $\odot .9534$ & -3.2898 \\
\hline $\operatorname{Si02}(\mathrm{aq})$ & ๑. . 0004206 & 25.27 & 1.0000 & -3.3761 \\
\hline $\mathrm{Ca}++$ & $\odot .0 \odot \odot 2778$ & 11.13 & $\odot .8299$ & -3.6373 \\
\hline $02(\mathrm{aq})$ & 0.0002460 & 7.871 & 1.0000 & -3.6090 \\
\hline $\mathrm{K}+$ & $7.548 e-0 \odot 5$ & 2.951 & 0.9527 & -4.1432 \\
\hline $\mathrm{Mg}++$ & $7.418 e-\odot \odot 5$ & 1.803 & $\odot .8338$ & -4.2086 \\
\hline sO4-- & $5.910 \mathrm{e}-005$ & 5.676 & 0.8257 & -4.3116 \\
\hline $\mathrm{co2}(\mathrm{aq})$ & $4.338 e-005$ & 1.909 & 1.0000 & -4.3627 \\
\hline $\mathrm{F}-$ & $3.012 \mathrm{e}-\odot \odot 5$ & 0.5721 & $\odot .9531$ & -4.5420 \\
\hline N03 - & $1.276 \mathrm{e}-005$ & $\odot .7911$ & $\odot .9527$ & -4.9152 \\
\hline $\mathrm{Cl}-$ & $8.520 \mathrm{e}-006$ & 0.3020 & $\odot .9527$ & -5.0906 \\
\hline Al02 - & $8.497 \mathrm{e}-006$ & 0.5011 & $\odot .9534$ & -5.0915 \\
\hline CO3-- & $5.114 \mathrm{e}-006$ & 0.3068 & 0.8268 & -5.3739 \\
\hline CaHCO3+ & $3.7 \odot 4 \mathrm{e}-\odot \odot 6$ & $\odot .3744$ & $\odot .9534$ & -5.4521 \\
\hline HSiO3- & $3.279 \mathrm{e}-\odot \odot 6$ & $\odot .2528$ & $\odot .9534$ & -5.5050 \\
\hline $\mathrm{Zn++}$ & $3.167 e-0 \odot 6$ & 0.2070 & $\odot .8299$ & -5.5804 \\
\hline Re04 - & $2.405 e-006$ & $\odot .6015$ & $\odot .9534$ & -5.6397 \\
\hline $\mathrm{CaCO} 3(\mathrm{aq})$ & $1.881 \mathrm{e}-006$ & 0.1883 & 1.0000 & -5.7256 \\
\hline CaSO4(aq) & $1.422 \mathrm{e}-0 \odot 6$ & 0.1935 & $1.0 \odot \odot \odot$ & -5.8471 \\
\hline $\operatorname{NaHCO} 3(\mathrm{aq})$ & 1. $\odot 92 \mathrm{e}-\odot \odot 6$ & ๑. 09170 & $1.00 \odot \odot$ & -5.9619 \\
\hline $\mathrm{MgHCO} 3+$ & $9.704 \mathrm{e}-0 \odot 7$ & ๑. 08278 & $\odot .9534$ & -6.0338 \\
\hline $\operatorname{MgSO} 4(\mathrm{aq})$ & $6.871 \mathrm{e}-0 \odot 7$ & $\odot .08269$ & 1.0000 & -6.1630 \\
\hline $\mathrm{OH}-$ & $5.332 e-0 \odot 7$ & 0.009067 & $\odot .9531$ & -6.2940 \\
\hline HAl02 (aq) & 3. $959 \mathrm{e}-0 \odot 7$ & $\odot .02375$ & 1.0000 & -6.4024 \\
\hline $\operatorname{MgC03}(\mathrm{aq})$ & $2.385 e-\odot \odot 7$ & ๑. 02010 & $1.0 \odot \odot \odot$ & -6.6225 \\
\hline NaHSiO3 (aq) & 8. $011 \mathrm{e}-008$ & ๑. .008016 & 1.0000 & -7.0963 \\
\hline $\mathrm{Ba}++$ & $7.370 \mathrm{e}-\odot \odot 8$ & ๑ . 01012 & 0.8278 & -7.2146 \\
\hline Mo04-- & $5.949 \mathrm{e}-\odot \odot 8$ & ๑ . ๑๑9513 & $\odot .8268$ & -7.3082 \\
\hline
\end{tabular}




\begin{tabular}{|c|c|c|c|c|}
\hline $\mathrm{MgF}+$ & $4.257 e-\odot \odot 8$ & ๑. .001843 & 0.9534 & -7.3916 \\
\hline $\mathrm{CaF}+$ & $3.286 e-008$ & 0.001941 & ๑. 9534 & -7.5040 \\
\hline KS04 - & $2.797 \mathrm{e}-\odot \odot 8$ & ๑. .๑378๑ & 0.9534 & -7.5740 \\
\hline ZnS04(aq) & $2.373 e-\odot \odot 8$ & ๑. . 003831 & $1.000 \odot$ & -7.6246 \\
\hline $\begin{array}{l}\mathrm{H}+ \\
\quad \text { (only sp }\end{array}$ & $\begin{array}{l}\text { 1. } 379 \mathrm{e}-\odot \odot 8 \\
8 \mathrm{molal} \text { lis }\end{array}$ & 1. $389 e-0 \odot 5$ & $\odot .9563$ & -7.8800 \\
\hline
\end{tabular}

Mineral saturation states

$\log \mathrm{Q} / \mathrm{K} \quad \log \mathrm{Q} / \mathrm{K}$

\begin{tabular}{|c|c|c|c|}
\hline Stilbite & $11.6999 \mathrm{~s} / \mathrm{sat}$ & Wairakite & $0.8802 \mathrm{~s} / \mathrm{sat}$ \\
\hline Muscovite & $10.7807 \mathrm{~s} / \mathrm{sat}$ & Saponite-Mg & $0.8508 \mathrm{~s} / \mathrm{sat}$ \\
\hline Beidellite-Ca & 8.0299s/sat & Witherite & $0.8463 \mathrm{~s} / \mathrm{sat}$ \\
\hline Beidellite-Mg & $7.9642 \mathrm{~s} / \mathrm{sat}$ & Quartz & $0.7557 \mathrm{~s} / \mathrm{sat}$ \\
\hline Illite & $7.9517 \mathrm{~s} / \mathrm{sat}$ & Albite_high & $\odot .7510 \mathrm{~s} / \mathrm{sat}$ \\
\hline Mesolite & $7.6646 \mathrm{~s} / \mathrm{sat}$ & Tridymite & $0.5581 \mathrm{~s} / \mathrm{sat}$ \\
\hline Paragonite & $7.5991 \mathrm{~s} / \mathrm{sat}$ & Sillimanite & $\odot .5564 \mathrm{~s} / \mathrm{sat}$ \\
\hline Beidellite-K & $7.5808 \mathrm{~s} / \mathrm{sat}$ & Saponite-K & $0.4667 \mathrm{~s} / \mathrm{sat}$ \\
\hline Beidellite-Na & $7.5084 \mathrm{~s} / \mathrm{sat}$ & Phlogopite & $0.4448 \mathrm{~s} / \mathrm{sat}$ \\
\hline Montmor-Mg & $7.1219 \mathrm{~s} / \mathrm{sat}$ & Saponite-Na & $0.3945 \mathrm{~s} / \mathrm{sat}$ \\
\hline Montmor-Ca & $7.1161 \mathrm{~s} / \mathrm{sat}$ & Cristobalite(alp & $\odot .1937 \mathrm{~s} / \mathrm{sat}$ \\
\hline Beidellite-H & 7.0109s/sat & Chalcedony & $0.1798 \mathrm{~s} / \mathrm{sat}$ \\
\hline Kaolinite & $6.9725 \mathrm{~s} / \mathrm{sat}$ & Coesite & -0.0675 \\
\hline Pyrophyllite & $6.8076 \mathrm{~s} / \mathrm{sat}$ & Saponite-H & -0.1032 \\
\hline Herschelite & $6.7708 \mathrm{~s} / \mathrm{sat}$ & Ice & -0.1194 \\
\hline Montmor-K & $6.7388 \mathrm{~s} / \mathrm{sat}$ & Cristobalite(bet & -0.2644 \\
\hline Montmor-Na & $6.6626 \mathrm{~s} / \mathrm{sat}$ & Dawsonite & -0.2692 \\
\hline Scolecite & $6.5220 \mathrm{~s} / \mathrm{sat}$ & Jadeite & -0.2763 \\
\hline Laumontite & $5.4507 \mathrm{~s} / \mathrm{sat}$ & Talc & -0.3094 \\
\hline Celadonite & 4.6950s/sat & Kalsilite & -0.3682 \\
\hline Margarite & $4.6471 \mathrm{~s} / \mathrm{sat}$ & Clinochlore-14A & -0.4410 \\
\hline Maximum_Microcli & $4.3754 \mathrm{~s} / \mathrm{sat}$ & Dolomite & -0.5113 \\
\hline K-Feldspar & $4.3742 \mathrm{~s} / \mathrm{sat}$ & Dolomite-ord & -0.5113 \\
\hline Amesite-14A & $3.5031 \mathrm{~s} / \mathrm{sat}$ & Calcite & -0.5495 \\
\hline Lawsonite & $3.4096 \mathrm{~s} / \mathrm{sat}$ & Analcime-K & $-\odot .5566$ \\
\hline Mordenite & $3.3308 \mathrm{~s} / \mathrm{sat}$ & Si02(am) & -0.5724 \\
\hline Sanidine_high & $3.1423 \mathrm{~s} / \mathrm{sat}$ & Aragonite & -0.6941 \\
\hline Diaspore & $2.9707 \mathrm{~s} / \mathrm{sat}$ & Smithsonite & -1.1212 \\
\hline Zn2Si04 & $2.7680 \mathrm{~s} / \mathrm{sat}$ & Anorthite & -1.1314 \\
\hline Boehmite & $2.5582 \mathrm{~s} / \mathrm{sat}$ & Zincite & -1.2989 \\
\hline Gibbsite & $2.3987 \mathrm{~s} / \mathrm{sat}$ & Monohydrocalcite & -1.3703 \\
\hline Albite_low & $2.1033 \mathrm{~s} / \mathrm{sat}$ & Barite & -1.4597 \\
\hline Albite & $2.1032 \mathrm{~s} / \mathrm{sat}$ & Magnesite & -1.6218 \\
\hline Corundum & $1.8424 \mathrm{~s} / \mathrm{sat}$ & $\mathrm{Zn}(\mathrm{OH}) 2($ gamma $)$ & -1.7036 \\
\hline Prehnite & $1.2676 \mathrm{~s} / \mathrm{sat}$ & $\mathrm{Zn}(\mathrm{OH}) 2$ (epsilon) & -1.7289 \\
\hline Kyanite & $1.2133 \mathrm{~s} / \mathrm{sat}$ & Analcime & -1.7374 \\
\hline Natrolite & $0.9913 \mathrm{~s} / \mathrm{sat}$ & $\mathrm{Zn}(\mathrm{OH}) 2($ beta $)$ & -2.0048 \\
\hline Clinozoisite & $\odot .9642 \mathrm{~s} / \mathrm{sat}$ & Dolomite-dis & -2.0931 \\
\hline Andalusite & ๑.9298s/sat & Nepheline & -2.4982 \\
\hline Zoisite & $\odot .9183 \mathrm{~s} / \mathrm{sat}$ & Fluorite & -2.6344 \\
\hline Saponite-Ca & ๑.9158s/sat & & \\
\hline (only minerals & ith $\log Q / K>$ & 3 listed) & \\
\hline Gases & fugacity & $\log$ fug. & \\
\hline \multicolumn{4}{|c|}{ - - - - - - - - - - - - - - - - - - - - - - - - - - - } \\
\hline $02(g)$ & $\odot .1755$ & $-\odot .756$ & \\
\hline $\mathrm{H} 2 \mathrm{O}(\mathrm{g})$ & 0.01903 & -1.721 & \\
\hline $\mathrm{C} 02(\mathrm{~g})$ & 0.001085 & -2.965 & \\
\hline $\mathrm{HF}(\mathrm{g})$ & $1.913 e-014$ & -13.718 & \\
\hline $\mathrm{HCl}(\mathrm{g})$ & $3.176 \mathrm{e}-020$ & -19.498 & \\
\hline N02 ( g ) & $3.235 e-021$ & -20.490 & \\
\hline $\mathrm{N} 2(\mathrm{~g})$ & $4.094 \mathrm{e}-022$ & -21.388 & \\
\hline NO $(g)$ & $3.235 \mathrm{e}-027$ & -26.490 & \\
\hline
\end{tabular}




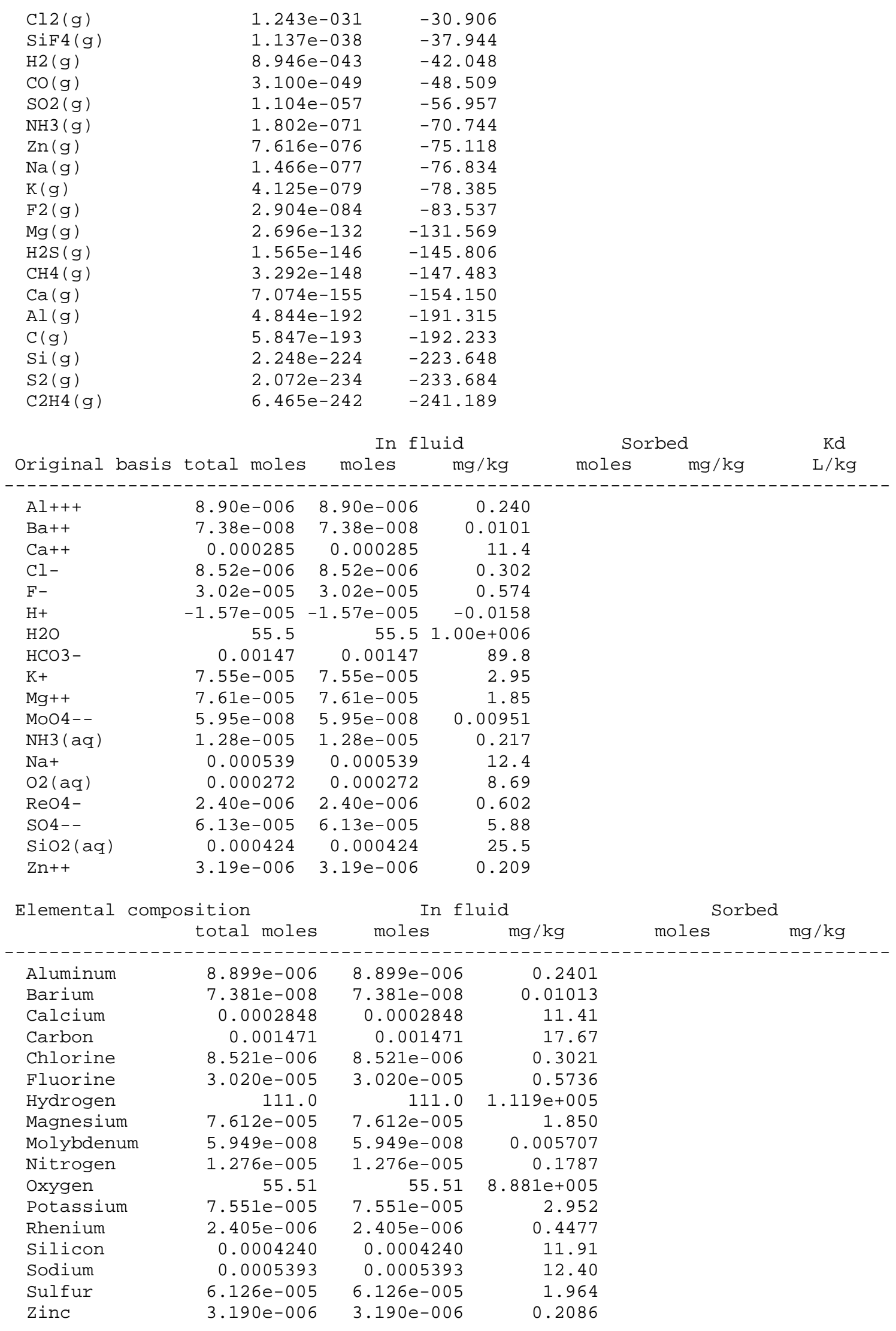




\section{Sample D10NTOP}

$\begin{array}{ll}\text { Step \# } \odot & \\ \text { Temperature }=20 . \odot \mathrm{C} \\ \text { pH }=7.800 \\ \text { Eh }=0.7656 \text { volts } \\ \text { Ionic strength } & = \\ \text { Charge imbalance } & = \\ \text { Activity of water } & = \\ \text { Solvent mass } & = \\ \text { Solution mass } & = \\ \text { Solution density } & = \\ \text { Chlorinity } & = \\ \text { Dissolved solids } & = \\ \text { Elect. conductivity } & = \\ \text { Hardness } & = \\ \text { carbonate } & = \\ \text { non-carbonate } & = \\ \text { Rock mass } & = \\ \text { Carbonate alkalinity= } & = \\ \text { Water type } & =\end{array}$

$\mathrm{Xi}=0.0000$

Pressure $=1.013$ bars

$\log \mathrm{f02}=-0.756$

pe $=13.1636$

0.002025

$-0.000370 \mathrm{eq} / \mathrm{kg}(-22.83 \%$ error $)$

$\odot .999999$

$1.000000 \mathrm{~kg}$

$1.000180 \mathrm{~kg}$

1. $016 \mathrm{~g} / \mathrm{cm} 3$

$0.000028 \mathrm{molal}$ $180 \mathrm{mg} / \mathrm{kg}$ sol' $\mathrm{n}$

$150.44 \mathrm{uS} / \mathrm{cm}$ (or umho/cm)

$32.15 \mathrm{mg} / \mathrm{kg}$ sol' $\mathrm{n}$ as $\mathrm{CaCO} 3$

$32.15 \mathrm{mg} / \mathrm{kg}$ sol'n as $\mathrm{CaCO} 3$

$0.00 \mathrm{mg} / \mathrm{kg}$ sol'n as $\mathrm{CaCO} 3$

$0.000000 \mathrm{~kg}$

$78.20 \mathrm{mg} / \mathrm{kg}$ sol'n as $\mathrm{CaCO} 3$ $\mathrm{Na}-\mathrm{HCO} 3$

No minerals in system.

\begin{tabular}{|c|c|c|c|c|}
\hline Aqueous species & molality & $\mathrm{mg} / \mathrm{kg}$ sol'n & act. coef. & $\log$ act \\
\hline - - - - - & 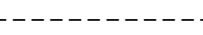 & $--_{-}--_{-}$ & - - - - - - & \\
\hline HCO3 - & 0.001546 & 94.32 & ๑. 9519 & -2.8322 \\
\hline $\mathrm{Na}+$ & $\odot .0 \odot \odot 7217$ & 16.59 & $\odot .9519$ & -3.1631 \\
\hline $\operatorname{SiO2}(\mathrm{aq})$ & 0.0004211 & 25.30 & 1.0000 & -3.3756 \\
\hline $02(\mathrm{aq})$ & $\odot .0002460$ & 7.871 & 1.0000 & -3.6090 \\
\hline $\mathrm{Ca}++$ & $\odot .0 \odot \odot 2389$ & 9.572 & $\odot .8249$ & -3.7055 \\
\hline SO4 - - & $8.412 \mathrm{e}-\odot \odot 5$ & 8.080 & 0.8204 & -4.1610 \\
\hline $\mathrm{K}+$ & $7.924 \mathrm{e}-005$ & 3.098 & 0.9512 & -4.1228 \\
\hline $\mathrm{Mg}++$ & $7.345 e-0 \odot 5$ & 1.785 & $\odot .8291$ & -4.2154 \\
\hline $\operatorname{co2}(\mathrm{aq})$ & $5.689 e-005$ & 2.503 & 1.0000 & -4.2450 \\
\hline $\mathrm{F}-$ & $3.622 \mathrm{e}-005$ & 0.6881 & 0.9515 & -4.4626 \\
\hline Cl - & $2.772 \mathrm{e}-\odot \odot 5$ & $\odot .9827$ & $\odot .9512$ & -4.5789 \\
\hline N03 - & 1. $255 \mathrm{e}-0 \odot 5$ & 0.7783 & 0.9512 & -4.9230 \\
\hline CO3-- & $4.669 e-0 \odot 6$ & 0.2801 & 0.8216 & -5.4161 \\
\hline CaHCO3+ & $3.459 \mathrm{e}-0 \odot 6$ & 0.3496 & $\odot .9519$ & -5.4825 \\
\hline $\mathrm{Zn++}$ & $2.755 e-0 \odot 6$ & 0.1801 & 0.8249 & -5.6435 \\
\hline HSiO3 - & $2.735 \mathrm{e}-\odot \odot 6$ & 0.2108 & $\odot .9519$ & -5.5844 \\
\hline Re04 - & $2.405 \mathrm{e}-0 \odot 6$ & 0.6015 & ๑. 9519 & -5.6404 \\
\hline CaSo4(aq) & $1.719 \mathrm{e}-\odot \odot 6$ & 0.2340 & 1.0000 & -5.7647 \\
\hline $\operatorname{NaHCO} 3(\mathrm{aq})$ & $1.594 \mathrm{e}-\odot \odot 6$ & 0.1339 & $1.0 \odot \odot \odot$ & -5.7974 \\
\hline $\mathrm{CaCO} 3(\mathrm{aq})$ & $1.459 \mathrm{e}-006$ & 0.1460 & 1.0000 & -5.8360 \\
\hline $\mathrm{MgHCO} 3+$ & 1. $.44 \mathrm{e}-\odot \odot 6$ & ๑. . 08904 & $\odot .9519$ & -6.0028 \\
\hline $\operatorname{MgSO} 4(\mathrm{aq})$ & $9.568 \mathrm{e}-0 \odot 7$ & 0.1151 & 1.0000 & -6.0192 \\
\hline $\mathrm{OH}-$ & $4.442 \mathrm{e}-\odot \odot 7$ & $\odot .007554$ & $\odot .9515$ & -6.3740 \\
\hline $\operatorname{MgC03}(\mathrm{aq})$ & $2.130 \mathrm{e}-\odot \odot 7$ & $\odot .01796$ & $1.00 \odot \odot$ & -6.6716 \\
\hline NaHSiO3(aq) & 8. 933e-๑०८ & ๑.๑०8939 & $1.00 \odot \odot$ & -7.0490 \\
\hline $\mathrm{Ba}++$ & $7.157 e-0 \odot 8$ & 0.009826 & 0.8227 & -7.2301 \\
\hline Mo04 - - & $5.949 \mathrm{e}-\odot \odot 8$ & $\odot .009513$ & 0.8216 & -7.3109 \\
\hline $\mathrm{MgF}+$ & $5.040 \mathrm{e}-008$ & 0.002182 & $\odot .9519$ & -7.3190 \\
\hline KSO4 - & $4.153 e-0 \odot 8$ & 0.005613 & $\odot .9519$ & -7.4030 \\
\hline CaF+ & $3.378 e-\odot \odot 8$ & ๑. 001995 & $\odot .9519$ & -7.4928 \\
\hline Znso4(aq) & $2.9 \odot 3 e-\odot \odot 8$ & ๑. . $\odot 4686$ & 1.0000 & -7.5372 \\
\hline $\mathrm{H}+$ & 1. $660 \mathrm{e}-\odot \odot 8$ & $1.672 \mathrm{e}-\odot \odot 5$ & $\odot .9549$ & $-7.80 \odot \odot$ \\
\hline $\mathrm{NaCO} 3-$ & 1. $045 \mathrm{e}-0 \odot 8$ & ๑. . 0008673 & ๑. 9519 & -8.0023 \\
\hline
\end{tabular}




\begin{tabular}{|c|c|c|c|}
\hline Mineral saturatic & \multicolumn{2}{|c|}{$\log Q / K$} & $\log Q / K$ \\
\hline \multicolumn{4}{|c|}{ Zn2Si04 $2.3224 \mathrm{~s} / \mathrm{sat}$ Aragonite $\quad-0.8045$} \\
\hline & & & -0.8045 \\
\hline Witherite & $0.7886 \mathrm{~s} / \mathrm{sat}$ & Talc & -0.8076 \\
\hline Quartz & $0.7562 \mathrm{~s} / \mathrm{sat}$ & Smithsonite & -1.2265 \\
\hline Tridymite & $0.5586 \mathrm{~s} / \mathrm{sat}$ & Barite & -1.3247 \\
\hline Cristobalite(alp & $0.1942 \mathrm{~s} / \mathrm{sat}$ & Monohydrocalcite & -1.4807 \\
\hline Chalcedony & $0.1803 \mathrm{~s} / \mathrm{sat}$ & Zincite & -1.5220 \\
\hline Coesite & -0.0670 & Magnesite & -1.6709 \\
\hline Ice & -0.1194 & $\mathrm{Zn}(\mathrm{OH}) 2($ gamma $)$ & -1.9267 \\
\hline Cristobalite (bet & -0.2638 & $\mathrm{Zn}(\mathrm{OH}) 2$ (epsilon) & -1.9520 \\
\hline Si02(am) & -0.5719 & $\mathrm{Zn}(\mathrm{OH}) 2($ beta $)$ & -2.2279 \\
\hline Calcite & $-\odot .6599$ & Dolomite-dis & -2.2526 \\
\hline Dolomite & -0.6708 & Fluorite & -2.5437 \\
\hline \multirow{2}{*}{\multicolumn{4}{|c|}{$\begin{array}{l}\text { Dolomite-ord }-0.6708 \\
\quad(\text { only minerals with } \log Q / K>-3 \text { listed) }\end{array}$}} \\
\hline & & & \\
\hline Gases & fugacity & $\log$ fug. & \\
\hline \multicolumn{4}{|c|}{ 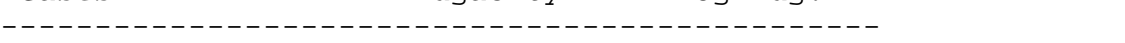 } \\
\hline $02(g)$ & 0.1755 & -0.756 & \\
\hline $\mathrm{H} 2 \mathrm{O}(\mathrm{g})$ & 0.01903 & -1.721 & \\
\hline $\operatorname{Co2}(\mathrm{g})$ & 0.001423 & -2.847 & \\
\hline$H F(g)$ & $2.762 \mathrm{e}-014$ & -13.559 & \\
\hline $\mathrm{HCl}(\mathrm{g})$ & $1.241 e-019$ & -18.906 & \\
\hline N02 ( g) & $3.821 e-021$ & -20.418 & \\
\hline $\mathrm{N} 2(\mathrm{~g})$ & $5.709 e-022$ & -21.243 & \\
\hline NO $(g)$ & $3.820 \mathrm{e}-027$ & -26.418 & \\
\hline $\operatorname{cl2}(g)$ & $1.896 e-030$ & -29.722 & \\
\hline $\operatorname{SiF} 4(g)$ & $4.942 e-038$ & -37.306 & \\
\hline $\mathrm{H} 2(\mathrm{~g})$ & $8.946 e-043$ & -42.048 & \\
\hline $\mathrm{CO}(\mathrm{g})$ & $4.066 e-049$ & -48.391 & \\
\hline S02(g) & $2.257 e-057$ & -56.647 & \\
\hline NH3 (g) & $2.128 e-071$ & -70.672 & \\
\hline $\mathrm{Zn}(\mathrm{g})$ & $4.556 e-076$ & -75.341 & \\
\hline $\mathrm{Na}(\mathrm{g})$ & $1.633 e-077$ & -76.787 & \\
\hline$K(g)$ & $3.596 \mathrm{e}-079$ & -78.444 & \\
\hline F2(g) & $6.051 e-084$ & -83.218 & \\
\hline$M g(g)$ & $1.836 e-132$ & -131.736 & \\
\hline $\mathrm{H} 2 \mathrm{~S}(\mathrm{~g})$ & $3.199 \mathrm{e}-146$ & -145.495 & \\
\hline $\mathrm{CH} 4(\mathrm{~g})$ & $4.317 e-148$ & -147.365 & \\
\hline $\mathrm{Ca}(\mathrm{g})$ & $4.183 e-155$ & -154.379 & \\
\hline$C(g)$ & $7.668 e-193$ & -192.115 & \\
\hline $\operatorname{Si}(g)$ & $2.251 e-224$ & -223.648 & \\
\hline $\mathrm{s} 2(\mathrm{~g})$ & $8.659 e-234$ & -233.063 & \\
\hline $\mathrm{C} 2 \mathrm{H} 4(\mathrm{~g})$ & $1.112 \mathrm{e}-241$ & -240.954 & \\
\hline
\end{tabular}

\begin{tabular}{|c|c|c|c|c|c|}
\hline \multirow[b]{2}{*}{ Original basis } & \multirow[b]{2}{*}{ total moles } & \multicolumn{2}{|c|}{ In fluid } & Sorbed & \multirow{2}{*}{$\begin{array}{c}\mathrm{Kd} \\
\mathrm{L} / \mathrm{kg}\end{array}$} \\
\hline & & moles & $\mathrm{mg} / \mathrm{kg}$ & moles $\quad \mathrm{mg} / \mathrm{kg}$ & \\
\hline & , & $-\cdots--\cdots$ & & & \\
\hline $\mathrm{Ba}++$ & $7.17 \mathrm{e}-0 \odot 8$ & $7.17 \mathrm{e}-\odot \odot 8$ & ๑.0०984 & & \\
\hline $\mathrm{Ca}++$ & $\odot .0 \odot \odot 246$ & $\odot .0 \odot \odot 246$ & 9.84 & & \\
\hline $\mathrm{Cl}-$ & $2.77 e-005$ & $2.77 \mathrm{e}-005$ & 0.983 & & \\
\hline $\mathrm{F}-$ & $3.63 e-005$ & $3.63 e-005$ & 0.690 & & \\
\hline $\mathrm{H}+$ & $3.47 \mathrm{e}-005$ & $3.47 \mathrm{e}-\odot \odot 5$ & $\odot .0350$ & & \\
\hline $\mathrm{H} 2 \mathrm{O}$ & 55.5 & 55.5 & 1. $\odot \odot e+\odot \odot 6$ & & \\
\hline HCO3- & 0.00162 & $\odot .0 \odot 162$ & 98.5 & & \\
\hline $\mathrm{K}+$ & $7.93 e-\odot \odot 5$ & $7.93 e-0 \odot 5$ & 3.10 & & \\
\hline $\mathrm{Mg}++$ & $7.57 e-0 \odot 5$ & $7.57 e-005$ & 1.84 & & \\
\hline Mo04 - - & $5.95 e-\odot \odot 8$ & $5.95 e-0 \odot 8$ & $\odot . \odot \odot 951$ & & \\
\hline $\mathrm{NH3}(\mathrm{aq})$ & $1.26 \mathrm{e}-005$ & $1.26 \mathrm{e}-005$ & 0.214 & & \\
\hline $\mathrm{Na}+$ & $\odot . \odot \odot \odot 723$ & $\odot .0 \odot \odot 723$ & 16.6 & & \\
\hline $02(\mathrm{aq})$ & $\odot .000271$ & $\odot .00 \odot 271$ & 8.67 & & \\
\hline
\end{tabular}




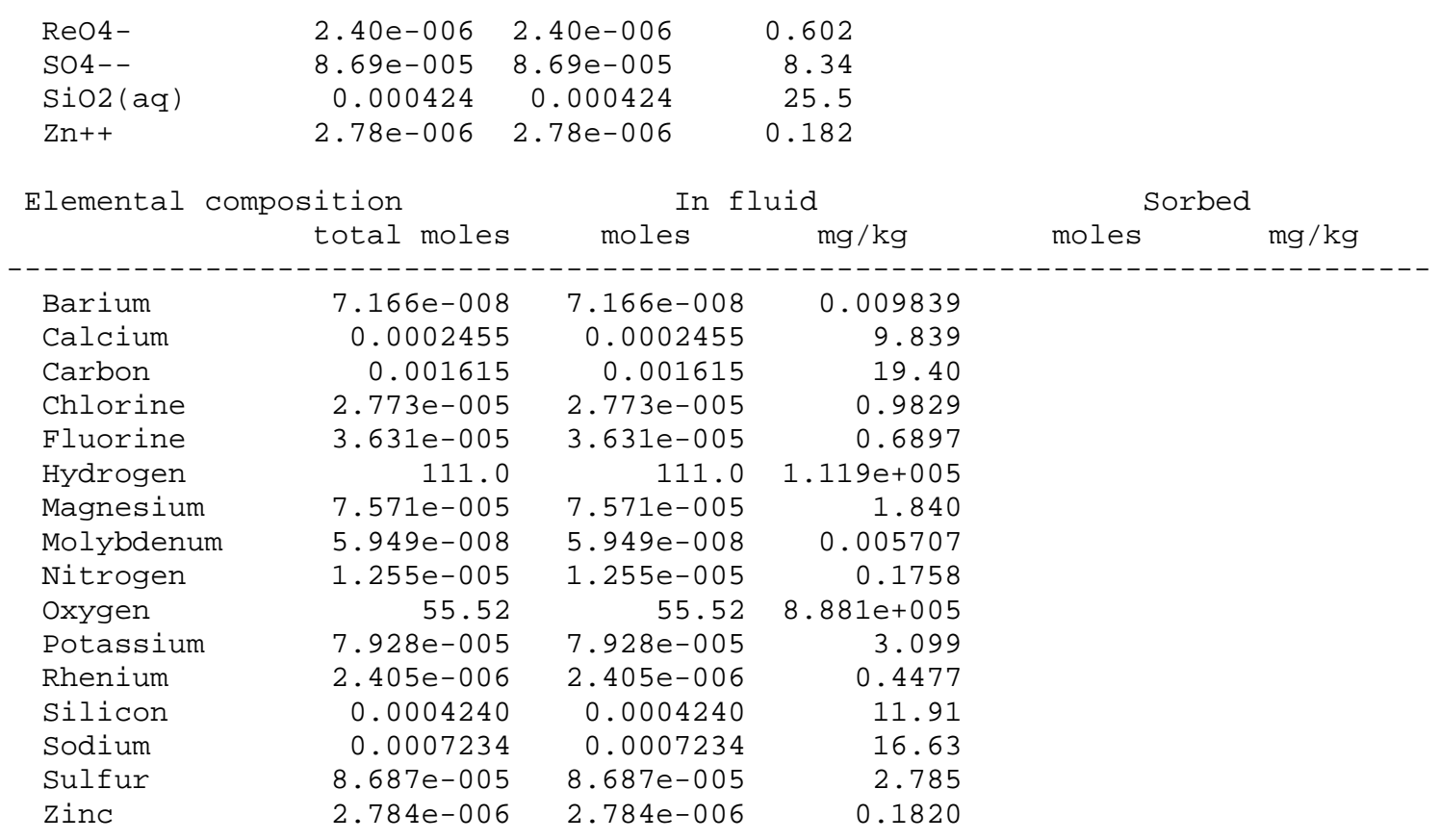




\section{Sample D10NETOP}

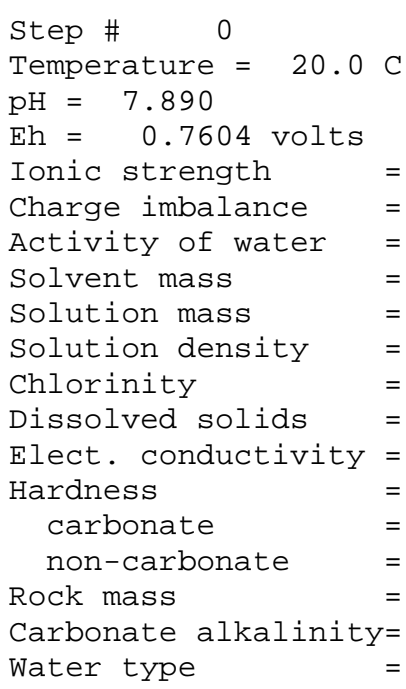

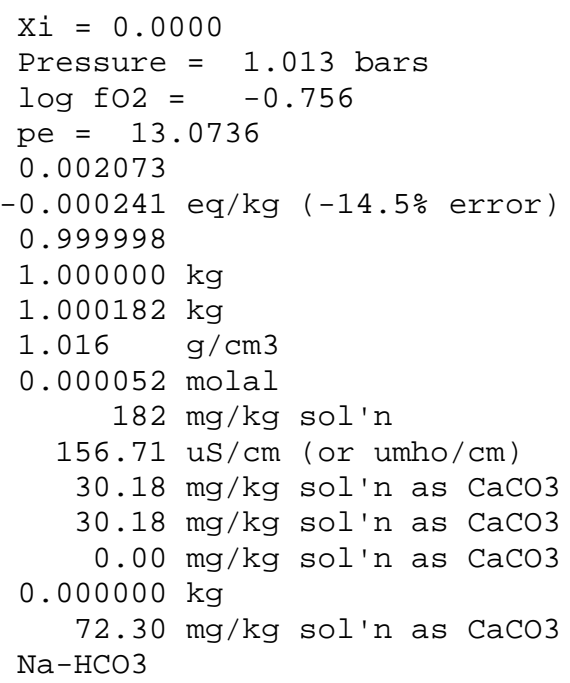

No minerals in system.

\begin{tabular}{|c|c|c|c|c|}
\hline Aqueous species & molality & $\mathrm{mg} / \mathrm{kg}$ sol'n & act. coef. & $\log$ act \\
\hline$----_{-}$ & $----\ldots$ & $-\ldots$ & $--_{-}-{ }_{-}$ & \\
\hline HCO3 - & $\odot .001427$ & 87.06 & $\odot .9513$ & -2.8672 \\
\hline $\mathrm{Na}+$ & 0.0008798 & 20.22 & 0.9513 & -3.0773 \\
\hline $\operatorname{Si02}(a q)$ & $\odot .00 \odot 4830$ & 29.02 & $1.00 \odot \odot$ & -3.3160 \\
\hline $02(\mathrm{aq})$ & $\odot .0 \odot \odot 2460$ & 7.871 & $1.00 \odot \odot$ & -3.6090 \\
\hline $\mathrm{Ca}++$ & ๑. . $00 \odot 2284$ & 9.150 & 0.8231 & -3.7259 \\
\hline SO4- - & $\odot .0 \odot \odot 1134$ & 10.89 & $\odot .8186$ & -4.0323 \\
\hline $\mathrm{K}+$ & $7.244 \mathrm{e}-005$ & 2.832 & 0.9507 & -4.1620 \\
\hline$M g++$ & $6.417 e-005$ & 1.559 & 0.8274 & -4.2749 \\
\hline Cl- & $5.217 \mathrm{e}-\odot \odot 5$ & 1.849 & $\odot .9507$ & -4.3045 \\
\hline $\mathrm{F}-$ & $4.584 \mathrm{e}-\odot \odot 5$ & $\odot .8708$ & $\odot .9510$ & -4.3605 \\
\hline $\mathrm{co2}(\mathrm{aq})$ & $4.266 e-0 \odot 5$ & 1.877 & 1.0000 & -4.3700 \\
\hline N03- & $1.298 e-005$ & $\odot .8048$ & $\odot .9507$ & -4.9086 \\
\hline CO3 - - & 5. $311 \mathrm{e}-006$ & $\odot .3186$ & $\odot .8198$ & -5.3611 \\
\hline HSi03 - & $3.862 \mathrm{e}-\odot \odot 6$ & $\odot .2977$ & $\odot .9513$ & -5.4349 \\
\hline CaHCO3+ & $3.046 e-006$ & 0.3078 & 0.9513 & -5.5380 \\
\hline Re04 - & $2.405 e-006$ & 0.6015 & 0.9513 & -5.6406 \\
\hline CaS04(aq) & $2.206 \mathrm{e}-\odot \odot 6$ & $\odot .30 \odot 2$ & $1.000 \odot$ & -5.6565 \\
\hline $\mathrm{NaHCO}(\mathrm{aq})$ & 1. $792 \mathrm{e}-006$ & $\odot .1505$ & 1.0000 & -5.7466 \\
\hline $\mathrm{CaCO} 3(\mathrm{aq})$ & $1.580 \mathrm{e}-006$ & $\odot .1581$ & 1.0000 & -5.8015 \\
\hline $\operatorname{MgSO} 4(\mathrm{aq})$ & $1.122 \mathrm{e}-0 \odot 6$ & 0.1350 & $1.0 \odot \odot \odot$ & -5.9500 \\
\hline $\mathrm{MgHCO}+$ & $8.401 \mathrm{e}-007$ & $\odot .07166$ & $\odot .9513$ & -6.0973 \\
\hline $\mathrm{OH}-$ & $5.468 \mathrm{e}-\odot \odot 7$ & ๑ . ๑๑9298 & $\odot .9510$ & -6.2840 \\
\hline $\operatorname{Mgco3}(\mathrm{aq})$ & $2.108 \mathrm{e}-\odot \odot 7$ & $\odot .01777$ & $1.0 \odot \odot \odot$ & -6.6761 \\
\hline NaHSiO3 (aq) & $1.536 \mathrm{e}-0 \odot 7$ & $\odot .01537$ & 1.0000 & -6.8137 \\
\hline $\mathrm{Ba}++$ & 6. 941e-๑๑8 & ๑. . 09530 & 0.8209 & -7.2443 \\
\hline Mo04 - - & $5.949 e-0 \odot 8$ & ๑. 009513 & 0.8198 & -7.3119 \\
\hline $\mathrm{MgF}+$ & $5.562 \mathrm{e}-0 \odot 8$ & $\odot .0 \odot 2408$ & $\odot .9513$ & -7.2764 \\
\hline KSO4 - & $5.107 \mathrm{e}-008$ & ๑. 006901 & $\odot .9513$ & -7.3135 \\
\hline $\mathrm{CaF}+$ & $4.078 e-0 \odot 8$ & $\odot .002409$ & 0.9513 & -7.4112 \\
\hline $\mathrm{NaCO} 3-$ & 1. $446 \mathrm{e}-\odot \odot 8$ & $\odot .0 \odot 12 \odot \odot$ & $\odot .9513$ & -7.8615 \\
\hline $\mathrm{H}+$ & 1. $350 \mathrm{e}-\odot \odot 8$ & $1.36 \odot \mathrm{e}-\odot \odot 5$ & 0.9545 & -7.8900 \\
\hline
\end{tabular}




\begin{tabular}{|c|c|c|c|}
\hline Mineral saturatio & \multicolumn{2}{|c|}{$\log Q / K$} & $\log Q / K$ \\
\hline \multicolumn{4}{|c|}{ 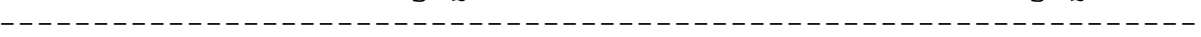 } \\
\hline Witherite & $0.8293 \mathrm{~s} / \mathrm{sat}$ & Calcite & -0.6254 \\
\hline Quartz & $\odot .8158 \mathrm{~s} / \mathrm{sat}$ & Dolomite & -0.6408 \\
\hline Tridymite & $\odot .6182 \mathrm{~s} / \mathrm{sat}$ & Dolomite-ord & -0.6408 \\
\hline Cristobalite(alp & $\odot .2538 \mathrm{~s} / \mathrm{sat}$ & Aragonite & -0.7700 \\
\hline Chalcedony & $\odot .2399 \mathrm{~s} / \mathrm{sat}$ & Barite & -1.2102 \\
\hline Coesite & 0.0075 & Monohydrocalcite & -1.4462 \\
\hline Ice & $\odot .1194$ & Magnesite & -1.6754 \\
\hline Cristobalite(bet & 0.2043 & Dolomite-dis & -2.2226 \\
\hline Talc & 0.2080 & Fluorite & -2.3601 \\
\hline $\begin{array}{l}\text { Si02 (am) } \\
\quad \text { (only minerals }\end{array}$ & $\begin{array}{l}0.5123 \\
h \log Q / K>\end{array}$ & 3 listed) & \\
\hline Gases & fugacity & $\log$ fug. & \\
\hline \multicolumn{4}{|c|}{ 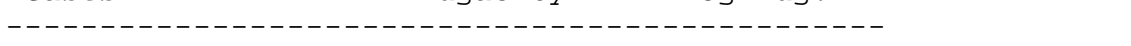 } \\
\hline $02(\mathrm{~g})$ & 0.1755 & $-\odot .756$ & \\
\hline $\mathrm{H} 20(\mathrm{~g})$ & $\odot .01903$ & -1.721 & \\
\hline $\operatorname{co2}(\mathrm{g})$ & 0.001067 & -2.972 & \\
\hline$H F(g)$ & $2.839 e-014$ & -13.547 & \\
\hline $\mathrm{HCl}(\mathrm{g})$ & $1.897 e-019$ & -18.722 & \\
\hline N02 $(g)$ & $3.210 \mathrm{e}-021$ & -20.494 & \\
\hline $\mathrm{N} 2(\mathrm{~g})$ & 4. $029 \mathrm{e}-022$ & -21.395 & \\
\hline NO $(g)$ & $3.209 e-027$ & -26.494 & \\
\hline $\operatorname{cl} 2(\mathrm{~g})$ & $4.430 \mathrm{e}-030$ & -29.354 & \\
\hline $\operatorname{SiF} 4(g)$ & $6.334 \mathrm{e}-038$ & -37.198 & \\
\hline $\mathrm{H} 2(\mathrm{~g})$ & $8.946 e-043$ & -42.048 & \\
\hline $\mathrm{CO}(\mathrm{g})$ & $3.049 e-\odot 49$ & -48.516 & \\
\hline S02(g) & $2.005 e-057$ & -56.698 & \\
\hline NH3 $(\mathrm{g})$ & $1.788 e-071$ & -70.748 & \\
\hline $\mathrm{Na}(\mathrm{g})$ & $2.448 e-077$ & -76.611 & \\
\hline$K(g)$ & $4.042 e-079$ & -78.393 & \\
\hline F2(g) & $6.397 e-084$ & -83.194 & \\
\hline $\operatorname{Mg}(\mathrm{g})$ & $2.423 e-132$ & -131.616 & \\
\hline $\mathrm{H} 2 \mathrm{~S}(\mathrm{~g})$ & $2.842 e-146$ & -145.546 & \\
\hline $\mathrm{CH} 4(\mathrm{~g})$ & $3.237 e-148$ & -147.490 & \\
\hline $\mathrm{Ca}(\mathrm{g})$ & $6.040 \mathrm{e}-155$ & -154.219 & \\
\hline$C(g)$ & $5.750 \mathrm{e}-193$ & -192.240 & \\
\hline $\operatorname{si}(g)$ & $2.582 \mathrm{e}-224$ & -223.588 & \\
\hline $\mathrm{s} 2(\mathrm{~g})$ & $6.838 e-234$ & -233.165 & \\
\hline $\mathrm{C} 2 \mathrm{H} 4(\mathrm{~g})$ & $6.252 e-242$ & -241.204 & \\
\hline
\end{tabular}

\begin{tabular}{|c|c|c|c|c|c|}
\hline \multirow[b]{2}{*}{ Original basis } & \multirow[b]{2}{*}{ total moles } & \multicolumn{2}{|c|}{ In fluid } & Sorbed & \multirow{2}{*}{$\begin{array}{c}\mathrm{Kd} \\
\mathrm{L} / \mathrm{kg}\end{array}$} \\
\hline & & moles & $\mathrm{mg} / \mathrm{kg}$ & moles $\quad \mathrm{mg} / \mathrm{kg}$ & \\
\hline $\mathrm{Ba}++$ & $6.95 e-008$ & $6.95 e-0 \odot 8$ & $\odot .00954$ & & \\
\hline $\mathrm{Ca}++$ & 0.000235 & 0.000235 & 9.43 & & \\
\hline $\mathrm{Cl}-$ & $5.22 e-005$ & $5.22 e-005$ & 1.85 & & \\
\hline $\mathrm{F}-$ & $4.59 \mathrm{e}-0 \odot 5$ & $4.59 \mathrm{e}-\odot \odot 5$ & 0.873 & & \\
\hline $\mathrm{H}+$ & $1.80 \mathrm{e}-005$ & $1.80 \mathrm{e}-005$ & 0.0182 & & \\
\hline $\mathrm{H} 2 \mathrm{O}$ & 55.5 & 55.5 & $1.00 \mathrm{e}+006$ & & \\
\hline $\mathrm{HCO} 3-$ & 0.00148 & 0.00148 & 90.4 & & \\
\hline $\mathrm{K}+$ & $7.25 e-005$ & $7.25 e-\odot \odot 5$ & 2.83 & & \\
\hline $\mathrm{Mg}++$ & $6.64 e-005$ & $6.64 \mathrm{e}-005$ & 1.61 & & \\
\hline Mo04 - - & $5.95 e-008$ & $5.95 e-008$ & $\odot .00951$ & & \\
\hline $\mathrm{NH} 3(\mathrm{aq})$ & $1.30 \mathrm{e}-005$ & $1.30 \mathrm{e}-005$ & 0.221 & & \\
\hline $\mathrm{Na}+$ & ๑. 000882 & ๑. . 000882 & 20.3 & & \\
\hline $02(\mathrm{aq})$ & 0.000272 & 0.000272 & 8.70 & & \\
\hline Re04- & $2.40 \mathrm{e}-006$ & $2.40 \mathrm{e}-006$ & 0.602 & & \\
\hline SO4 - - & $\odot .000117$ & 0.000117 & 11.2 & & \\
\hline $\operatorname{Si02}(\mathrm{aq})$ & 0.000487 & 0.000487 & 29.3 & & \\
\hline
\end{tabular}




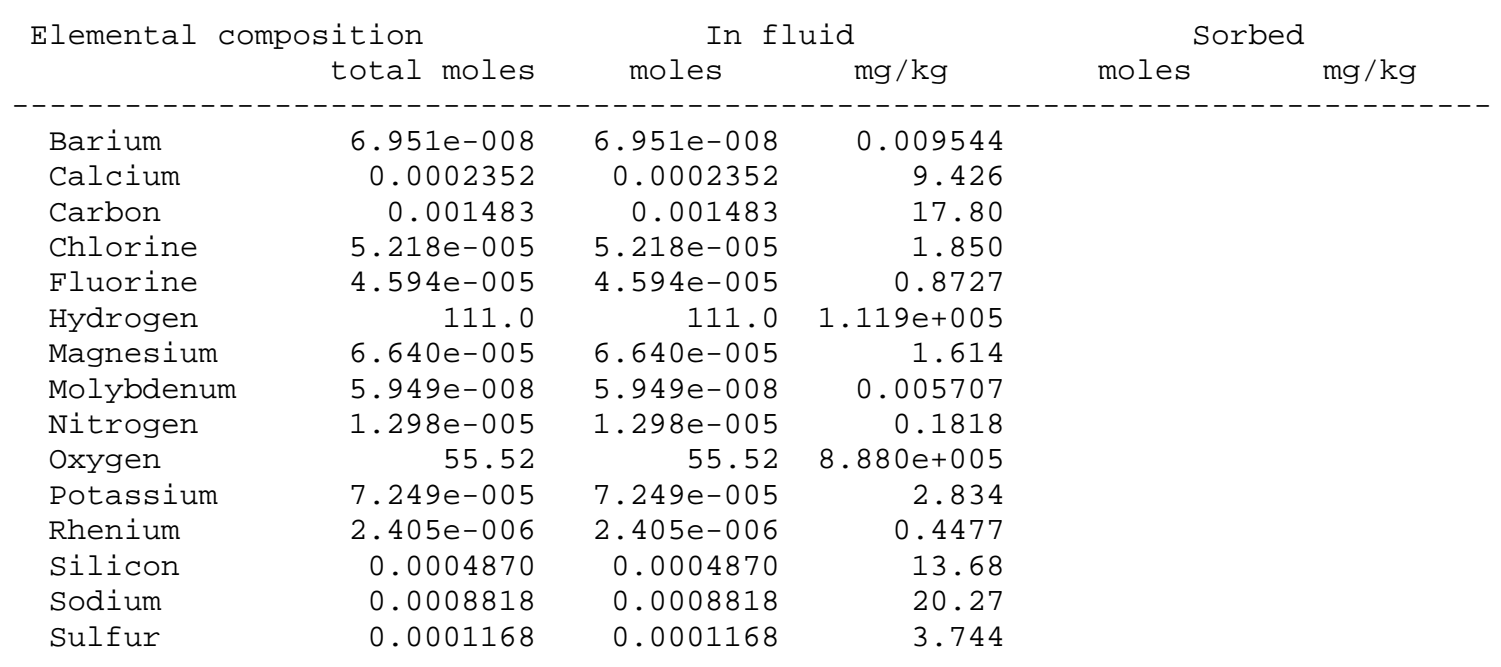




\section{Sample D10STOP}

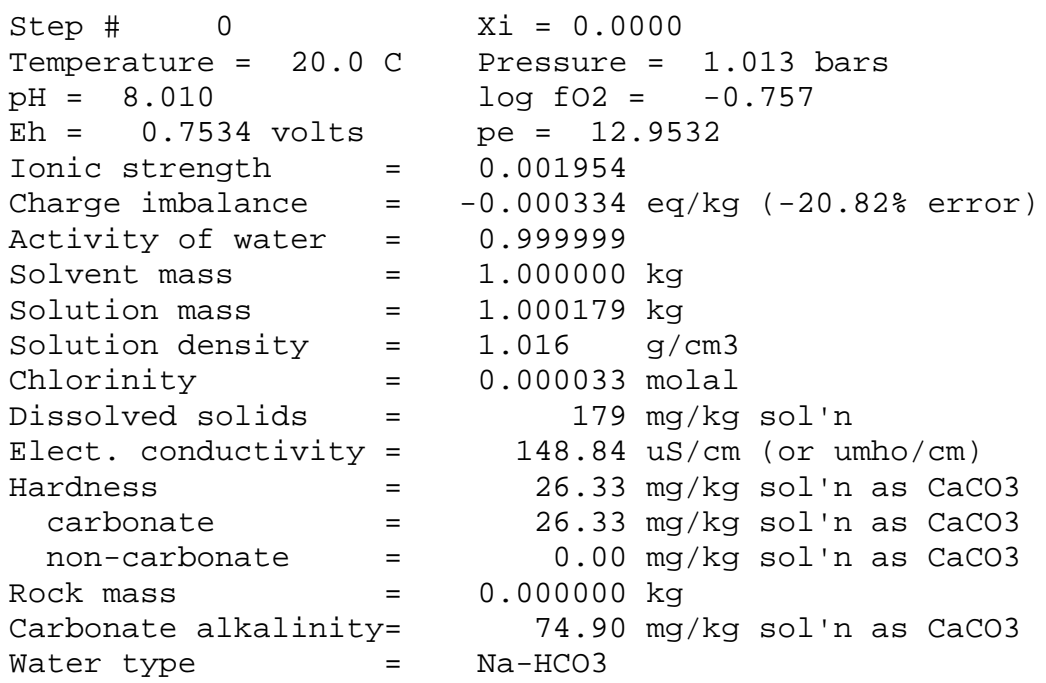

No minerals in system.

\begin{tabular}{|c|c|c|c|c|}
\hline Aqueous species & molality & $\mathrm{mg} / \mathrm{kg}$ sol'n & act. coef. & $\log$ act \\
\hline.--- & & ------ & 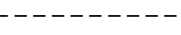 & ---- \\
\hline HCO3 - & $\odot .0 \odot 1475$ & 89.99 & $\odot .9526$ & -2.8522 \\
\hline $\mathrm{Na}+$ & ๑. .๑๑8541 & 19.63 & $\odot .9526$ & -3.0896 \\
\hline $\operatorname{Si02}(\mathrm{aq})$ & 0.0004610 & 27.69 & 1.0000 & -3.3363 \\
\hline $02(\mathrm{aq})$ & ๑. . 0002451 & 7.841 & 1.0000 & -3.6107 \\
\hline $\mathrm{Ca}++$ & $\odot .0 \odot \odot 2 \odot 22$ & 8.101 & $\odot .8274$ & -3.7765 \\
\hline SO4 - - & $8.826 e-005$ & 8.477 & 0.8232 & -4.1388 \\
\hline $\mathrm{K}+$ & $6.817 e-0 \odot 5$ & 2.665 & $\odot .9520$ & -4.1878 \\
\hline$M g++$ & $5.293 e-005$ & 1.286 & 0.8315 & -4.3565 \\
\hline $\mathrm{F}-$ & $4.617 \mathrm{e}-005$ & $\odot .8770$ & 0.9523 & -4.3569 \\
\hline $\mathrm{co2}(\mathrm{aq})$ & $3.350 \mathrm{e}-\odot \odot 5$ & 1.474 & $1.000 \odot$ & -4.4750 \\
\hline $\mathrm{Cl}-$ & $3.330 \mathrm{e}-005$ & 1.180 & 0.9520 & -4.4989 \\
\hline N03 - & $9.665 e-006$ & $\odot .5992$ & 0.9520 & -5.0361 \\
\hline CO3-- & $7.208 e-\odot \odot 6$ & $\odot .4324$ & 0.8242 & -5.2262 \\
\hline Al02 - & $6.519 \mathrm{e}-006$ & $\odot .3844$ & 0.9526 & -5.2069 \\
\hline HSiO3- & $4.852 \mathrm{e}-006$ & 0.3740 & 0.9526 & -5.3352 \\
\hline $\mathrm{Fe}(\mathrm{OH}) 3(\mathrm{aq})$ & $3.806 \mathrm{e}-\odot \odot 6$ & $\odot .4067$ & $1.0 \odot \odot \odot$ & -5.4195 \\
\hline $\mathrm{CaHCO}+$ & $2.802 \mathrm{e}-0 \odot 6$ & 0.2832 & 0.9526 & -5.5736 \\
\hline Re04 - & $2.405 e-006$ & $\odot .6015$ & $\odot .9526$ & -5.6400 \\
\hline Сac03 $(\mathrm{aq})$ & 1. $918 \mathrm{e}-\odot \odot 6$ & $\odot .1920$ & $1.00 \odot \odot$ & -5.7171 \\
\hline $\mathrm{NaHCO} 3(\mathrm{aq})$ & 1. $803 e-006$ & $\odot .1515$ & 1.0000 & -5.7439 \\
\hline CaSO4(aq) & $1.536 \mathrm{e}-006$ & ๑. 2091 & 1.0000 & -5.8136 \\
\hline $\operatorname{MgSO} 4(\mathrm{aq})$ & $7.279 \mathrm{e}-0 \odot 7$ & 0.08760 & 1.0000 & -6.1380 \\
\hline $\mathrm{OH}-$ & $7.199 \mathrm{e}-007$ & 0.01224 & 0.9523 & -6.1640 \\
\hline MgHCO3+ & $7.198 \mathrm{e}-007$ & 0.06140 & $\odot .9526$ & -6.1639 \\
\hline $\operatorname{MgC0} 3(\mathrm{aq})$ & $2.384 \mathrm{e}-0 \odot 7$ & 0.02010 & 1.0000 & -6.6226 \\
\hline HAl02 (aq) & $2.250 \mathrm{e}-\odot \odot 7$ & 0.01350 & $1.0 \odot \odot \odot$ & -6.6478 \\
\hline NaHSiO3(aq) & $1.878 \mathrm{e}-0 \odot 7$ & ๑. 01879 & 1.0000 & -6.7262 \\
\hline $\mathrm{Ba}++$ & $7.080 \mathrm{e}-008$ & 0.009721 & 0.8253 & -7.2333 \\
\hline Mo04 - - & $5.949 \mathrm{e}-\odot \odot 8$ & ๑ . ๑๑9513 & $\odot .8242$ & -7.3095 \\
\hline MgF+ & $4.643 e-0 \odot 8$ & ๑ . 002010 & 0.9526 & -7.3543 \\
\hline KSO4 - & 3. 761e-0०8 & 0.005083 & $\odot .9526$ & -7.4458 \\
\hline $\mathrm{CaF}+$ & $3.655 \mathrm{e}-\odot \odot 8$ & ๑ . ๑๑2159 & $\odot .9526$ & -7.4581 \\
\hline $\mathrm{NaCO} 3-$ & 1.915e-008 & ๑ . 001590 & $\odot .9526$ & -7.7388 \\
\hline $\mathrm{H}+$ & $1.023 e-0 \odot 8$ & $1.031 \mathrm{e}-005$ & ๑. 9556 & -8.0100 \\
\hline
\end{tabular}

(only species > 1e-8 molal listed) 


\begin{tabular}{|c|c|c|c|}
\hline & $\log \mathrm{Q} / \mathrm{K}$ & & $\log Q / K$ \\
\hline & & & \\
\hline Nontronite-ca & $17.9043 \mathrm{~s} / \mathrm{sat}$ & Gibbsite & $2.1533 \mathrm{~s} / \mathrm{sat}$ \\
\hline Nontronite-Mg & $17.8373 \mathrm{~s} / \mathrm{sat}$ & Smectite-low-Fe- & $1.8282 \mathrm{~s} / \mathrm{sat}$ \\
\hline Nontronite-Na & $17.4720 \mathrm{~s} / \mathrm{sat}$ & Corundum & $1.3515 \mathrm{~s} / \mathrm{sat}$ \\
\hline Nontronite-K & $17.4634 \mathrm{~s} / \mathrm{sat}$ & Saponite-Ca & $1.3374 \mathrm{~s} / \mathrm{sat}$ \\
\hline Nontronite-H & $16.8653 \mathrm{~s} / \mathrm{sat}$ & Natrolite & $1.2804 \mathrm{~s} / \mathrm{sat}$ \\
\hline Clinoptilolite- & $15.3853 \mathrm{~s} / \mathrm{sat}$ & Saponite-Mg & $1.2710 \mathrm{~s} / \mathrm{sat}$ \\
\hline Clinoptilolite-C & $15.3563 \mathrm{~s} / \mathrm{sat}$ & Prehnite & $1.1377 \mathrm{~s} / \mathrm{sat}$ \\
\hline Hematite & $12.6694 \mathrm{~s} / \mathrm{sat}$ & Witherite & $0.9753 \mathrm{~s} / \mathrm{sat}$ \\
\hline Clinoptilolite-K & $11.7580 \mathrm{~s} / \mathrm{sat}$ & Albite_high & $0.9553 \mathrm{~s} / \mathrm{sat}$ \\
\hline Clinoptilolite-r & $11.6482 \mathrm{~s} / \mathrm{sat}$ & Saponite-Na & $0.9051 \mathrm{~s} / \mathrm{sat}$ \\
\hline Stilbite & $11.6048 \mathrm{~s} / \mathrm{sat}$ & Saponite-K & $0.8965 \mathrm{~s} / \mathrm{sat}$ \\
\hline Clinoptilolite- & $11.5536 \mathrm{~s} / \mathrm{sat}$ & Quartz & $0.7955 \mathrm{~s} / \mathrm{sat}$ \\
\hline Clinoptilolite-N & $11.5528 \mathrm{~s} / \mathrm{sat}$ & Kyanite & $0.7623 \mathrm{~s} / \mathrm{sat}$ \\
\hline Muscovite & $10.2493 \mathrm{~s} / \mathrm{sat}$ & Magnetite & $0.7441 \mathrm{~s} / \mathrm{sat}$ \\
\hline Beidellite-Ca & $7.6240 \mathrm{~s} / \mathrm{sat}$ & Phlogopite & $0.7408 \mathrm{~s} / \mathrm{sat}$ \\
\hline Illite & $7.6058 \mathrm{~s} / \mathrm{sat}$ & Wairakite & $0.6694 \mathrm{~s} / \mathrm{sat}$ \\
\hline Mesolite & $7.5986 \mathrm{~s} / \mathrm{sat}$ & Tridymite & $0.5979 \mathrm{~s} / \mathrm{sat}$ \\
\hline Beidellite-Mg & $7.5570 \mathrm{~s} / \mathrm{sat}$ & Clinozoisite & $0.5889 \mathrm{~s} / \mathrm{sat}$ \\
\hline Epidote-ord & $7.4976 \mathrm{~s} / \mathrm{sat}$ & Zoisite & $0.5430 \mathrm{~s} / \mathrm{sat}$ \\
\hline Epidote & $7.4972 \mathrm{~s} / \mathrm{sat}$ & Andalusite & $0.4788 \mathrm{~s} / \mathrm{sat}$ \\
\hline Paragonite & $7.3126 \mathrm{~s} / \mathrm{sat}$ & Saponite-H & $0.2985 \mathrm{~s} / \mathrm{sat}$ \\
\hline Beidellite-Na & $7.1917 \mathrm{~s} / \mathrm{sat}$ & Cristobalite(alp & $0.2335 \mathrm{~s} / \mathrm{sat}$ \\
\hline Beidellite-K & $7.1832 \mathrm{~s} / \mathrm{sat}$ & Chalcedony & $0.2196 \mathrm{~s} / \mathrm{sat}$ \\
\hline Herschelite & $6.9531 \mathrm{~s} / \mathrm{sat}$ & Talc & $0.1863 \mathrm{~s} / \mathrm{sat}$ \\
\hline Montmor-Mg & $6.9268 \mathrm{~s} / \mathrm{sat}$ & Sillimanite & $0.1053 \mathrm{~s} / \mathrm{sat}$ \\
\hline Montmor-Ca & $6.9224 \mathrm{~s} / \mathrm{sat}$ & Coesite & -0.0277 \\
\hline Beidellite-H & $6.5851 \mathrm{~s} / \mathrm{sat}$ & Jadeite & -0.1118 \\
\hline Kaolinite & $6.5613 \mathrm{~s} / \mathrm{sat}$ & Ice & -0.1194 \\
\hline Montmor- $\mathrm{Na}$ & $6.5579 \mathrm{~s} / \mathrm{sat}$ & Cristobalite(bet & -0.2246 \\
\hline Montmor-K & $6.5534 \mathrm{~s} / \mathrm{sat}$ & Clinochlore-14A & -0.2515 \\
\hline Pyrophyllite & $6.4760 \mathrm{~s} / \mathrm{sat}$ & Dawsonite & -0.2966 \\
\hline Scolecite & $6.2713 \mathrm{~s} / \mathrm{sat}$ & Kalsilite & $-\odot .4884$ \\
\hline Goethite & $5.8602 \mathrm{~s} / \mathrm{sat}$ & Dolomite & -0.5029 \\
\hline Andradite & $5.7370 \mathrm{~s} / \mathrm{sat}$ & Dolomite-ord & -0.5029 \\
\hline Laumontite & $5.2398 \mathrm{~s} / \mathrm{sat}$ & Si02(am) & -0.5326 \\
\hline Celadonite & $4.8064 \mathrm{~s} / \mathrm{sat}$ & Calcite & -0.5410 \\
\hline Maximum_Microcli & $4.3348 \mathrm{~s} / \mathrm{sat}$ & Analcime-K & $-\odot .6290$ \\
\hline K-Feldspar & $4.3337 \mathrm{~s} / \mathrm{sat}$ & Aragonite & -0.6856 \\
\hline Margarite & $3.8658 \mathrm{~s} / \mathrm{sat}$ & Smectite-high-Fe & -1.1703 \\
\hline Mordenite & $3.4557 \mathrm{~s} / \mathrm{sat}$ & Barite & -1.3057 \\
\hline Lawsonite & $3.1192 \mathrm{~s} / \mathrm{sat}$ & Monohydrocalcite & -1.3618 \\
\hline Sanidine_high & $3.1017 \mathrm{~s} / \mathrm{sat}$ & Anorthite & -1.4219 \\
\hline Ferrite-Ca & $3.0874 \mathrm{~s} / \mathrm{sat}$ & Analcime & -1.5747 \\
\hline Amesite-14A & $3.0498 \mathrm{~s} / \mathrm{sat}$ & Magnesite & -1.6219 \\
\hline Ferrite-Mg & $2.9298 \mathrm{~s} / \mathrm{sat}$ & Dolomite-dis & -2.0847 \\
\hline Diaspore & $2.7252 \mathrm{~s} / \mathrm{sat}$ & Nepheline & -2.3735 \\
\hline Boehmite & $2.3128 \mathrm{~s} / \mathrm{sat}$ & Fluorite & -2.4033 \\
\hline Albite_low & $2.3076 \mathrm{~s} / \mathrm{sat}$ & $\mathrm{Fe}(\mathrm{OH}) 3$ & -2.8277 \\
\hline Albite & $2.3075 \mathrm{~s} / \mathrm{sat}$ & & \\
\hline (only minerals & with $\log Q / K>$ & -3 listed) & \\
\hline Gases & fugacity & $\log$ fug. & \\
\hline 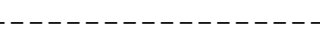 & 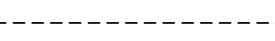 & & \\
\hline $02(g)$ & 0.1748 & -0.757 & \\
\hline $\mathrm{H} 2 \mathrm{O}(\mathrm{g})$ & 0.01903 & -1.721 & \\
\hline $\operatorname{Co2}(\mathrm{g})$ & $\odot .0008377$ & -3.077 & \\
\hline $\mathrm{HF}(\mathrm{g})$ & $2.172 \mathrm{e}-014$ & -13.663 & \\
\hline $\mathrm{HCl}(\mathrm{g})$ & $9.196 \mathrm{e}-020$ & -19.036 & \\
\hline N02 $(g)$ & $1.817 e-021$ & -20.741 & \\
\hline N2 (g) & 1. $301 \mathrm{e}-022$ & -21.886 & \\
\hline
\end{tabular}




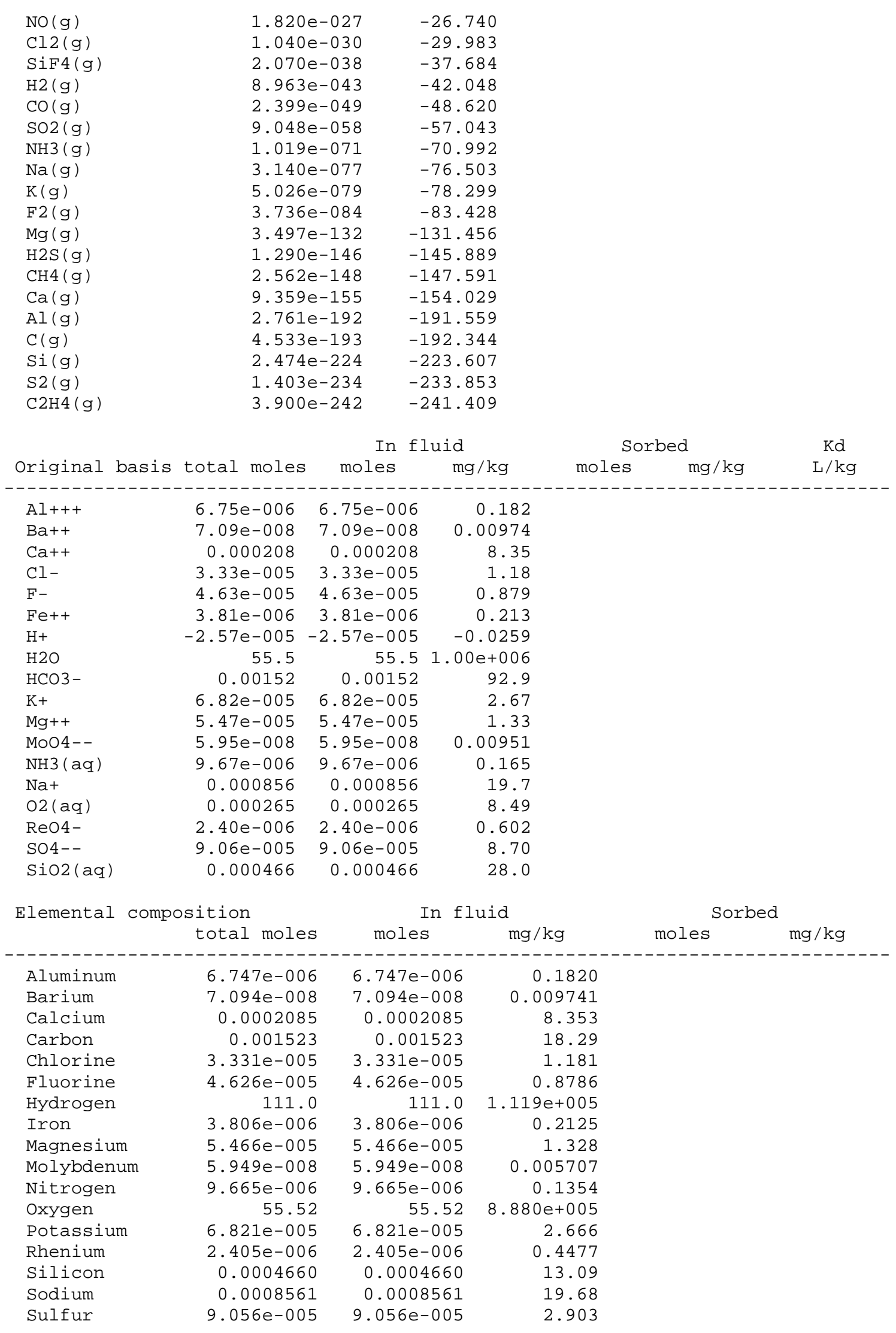




\section{Sample D10WTOP}

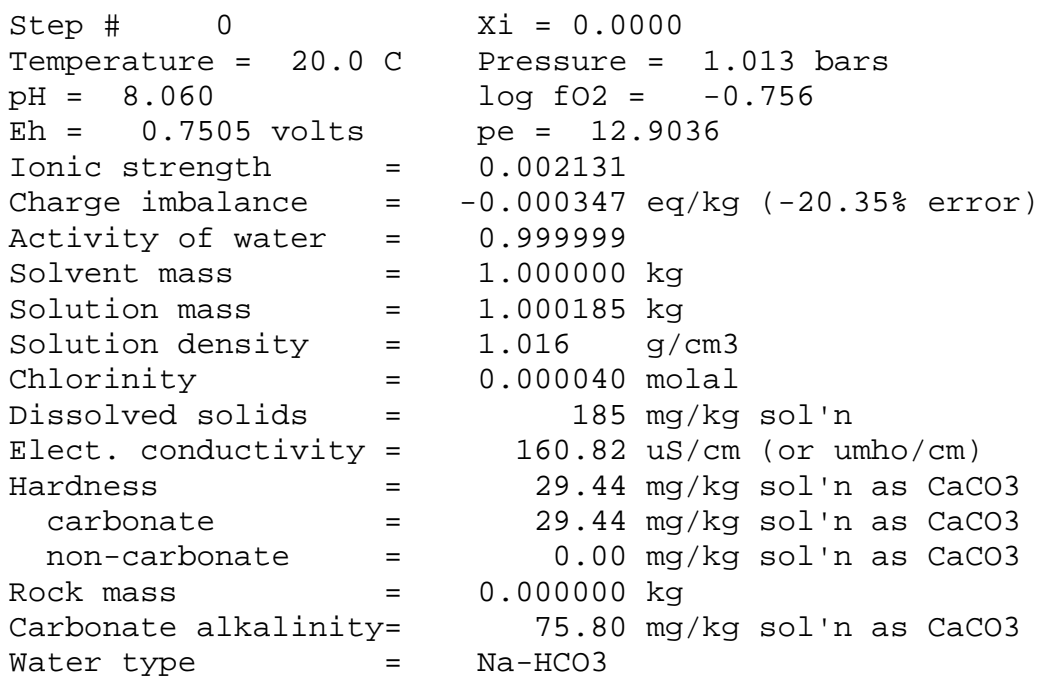

No minerals in system.

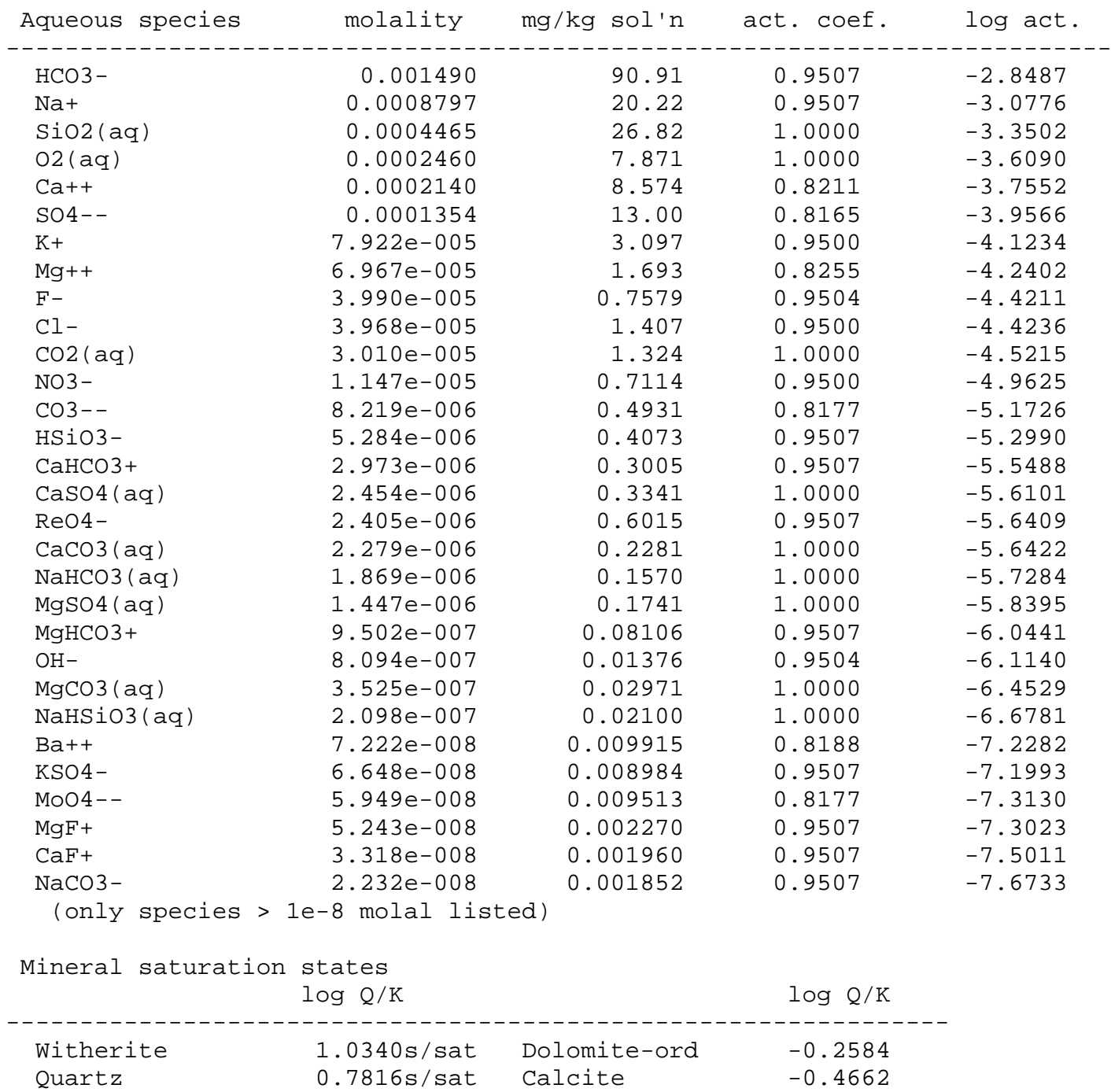




$\begin{array}{lllr}\text { Talc } & 0.7796 \mathrm{~s} / \mathrm{sat} & \text { Si02(am) } & -0.5465 \\ \text { Tridymite } & 0.5840 \mathrm{~s} / \mathrm{sat} & \text { Aragonite } & -0.6108 \\ \text { Cristobalite(alp } & 0.2196 \mathrm{~s} / \mathrm{sat} & \text { Barite } & -1.1183 \\ \text { Chalcedony } & 0.2057 \mathrm{~s} / \mathrm{sat} & \text { Monohydrocalcite } & -1.2869 \\ \text { Coesite } & -0.0416 & \text { Magnesite } & -1.4522 \\ \text { Ice } & -0.1194 & \text { Dolomite-dis } & -1.8402 \\ \text { Cristobalite(bet } & -0.2384 & \text { Fluorite } & -2.5106 \\ \text { Dolomite } & -0.2584 & \text { Chrysotile } & -2.7843\end{array}$

(only minerals with $\log Q / K>-3$ listed)

\begin{tabular}{lcc} 
Gases & fugacity & log fug \\
\hline O2 $(g)$ & 0.1755 & -0.756 \\
H2O $(\mathrm{g})$ & 0.01903 & -1.721 \\
$\mathrm{C} 2(\mathrm{~g})$ & $0.00 \odot 7527$ & -3.123 \\
$\mathrm{HF}(\mathrm{g})$ & $1.670 \mathrm{e}-014$ & -13.777 \\
$\mathrm{HCl}(\mathrm{g})$ & $9.747 \mathrm{e}-020$ & -19.011 \\
$\mathrm{NO2}(\mathrm{g})$ & $1.917 \mathrm{e}-021$ & -20.717 \\
$\mathrm{~N} 2(\mathrm{~g})$ & $1.437 \mathrm{e}-022$ & -21.843 \\
$\mathrm{NO}(\mathrm{g})$ & $1.916 \mathrm{e}-027$ & -26.717 \\
$\mathrm{C} 12(\mathrm{~g})$ & $1.170 \mathrm{e}-030$ & -29.932 \\
$\mathrm{SiF}(\mathrm{g})$ & $7.000 \mathrm{e}-039$ & -38.155 \\
$\mathrm{H} 2(\mathrm{~g})$ & $8.946 \mathrm{e}-043$ & -42.048 \\
$\mathrm{CO}(\mathrm{g})$ & $2.151 \mathrm{e}-049$ & -48.667 \\
$\mathrm{~S} 2(\mathrm{~g})$ & $1.091 \mathrm{e}-057$ & -56.962 \\
$\mathrm{NH}(\mathrm{g})$ & $1.068 \mathrm{e}-071$ & -70.972 \\
$\mathrm{Na}(\mathrm{g})$ & $3.618 \mathrm{e}-077$ & -76.442 \\
$\mathrm{~K}(\mathrm{~g})$ & $6.533 \mathrm{e}-079$ & -78.185 \\
$\mathrm{~F} 2(\mathrm{~g})$ & $2.212 \mathrm{e}-084$ & -83.655 \\
$\mathrm{Mg}(\mathrm{g})$ & $5.743 \mathrm{e}-132$ & -131.241 \\
$\mathrm{H} 2 \mathrm{~S}(\mathrm{~g})$ & $1.547 \mathrm{e}-146$ & -145.811 \\
$\mathrm{CH} 4(\mathrm{~g})$ & $2.284 \mathrm{e}-148$ & -147.641 \\
$\mathrm{Ca}(\mathrm{g})$ & $1.235 \mathrm{e}-154$ & -153.908 \\
$\mathrm{C}(\mathrm{g})$ & $4.057 \mathrm{e}-193$ & -192.392 \\
$\mathrm{Si}(\mathrm{g})$ & $2.387 \mathrm{e}-224$ & -223.622 \\
$\mathrm{~S} 2(\mathrm{~g})$ & $2.025 \mathrm{e}-234$ & -233.694 \\
$\mathrm{C} 2 \mathrm{H} 4(\mathrm{~g})$ & $3.112 \mathrm{e}-242$ & -241.507 \\
& & \\
& &
\end{tabular}

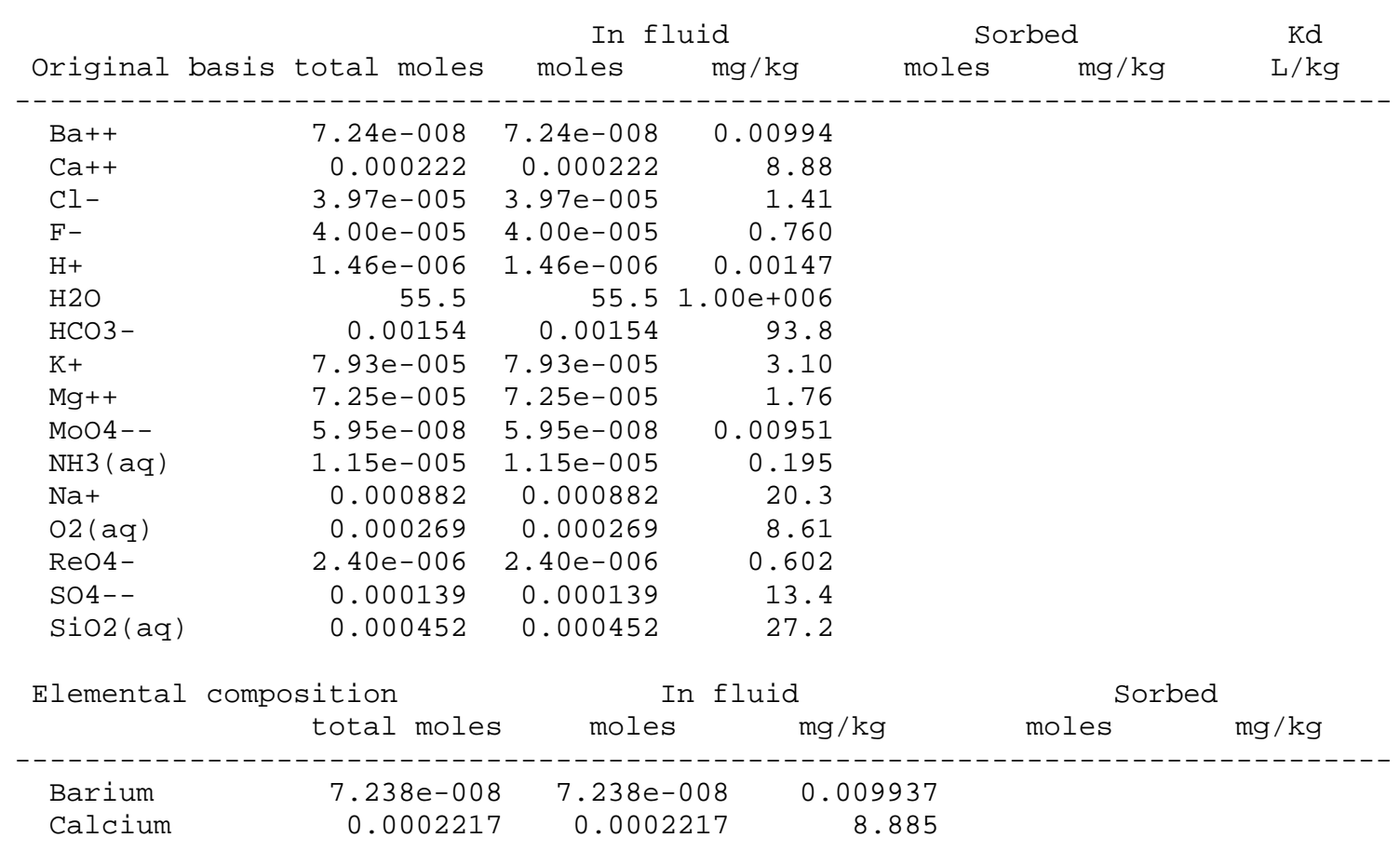




$\begin{array}{lrrr}\text { Carbon } & & & \\ \text { Chlorine } & 3.969 \mathrm{e}-005 & 3.969 \mathrm{e}-005 & 18.46 \\ \text { Fluorine } & 3.999 \mathrm{e}-005 & 3.999 \mathrm{e}-005 & 1.407 \\ \text { Hydrogen } & 111.0 & 111.0 & 1.119 \mathrm{e}+005 \\ \text { Magnesium } & 7.247 \mathrm{e}-005 & 7.247 \mathrm{e}-005 & 1.761 \\ \text { Molybdenum } & 5.949 \mathrm{e}-008 & 5.949 \mathrm{e}-008 & 0.005707 \\ \text { Nitrogen } & 1.147 \mathrm{e}-005 & 1.147 \mathrm{e}-005 & 0.1607 \\ \text { Oxygen } & 55.52 & 55.52 & 8.880 \mathrm{e}+005 \\ \text { Potassium } & 7.928 \mathrm{e}-005 & 7.928 \mathrm{e}-005 & 3.099 \\ \text { Rhenium } & 2.405 \mathrm{e}-006 & 2.405 \mathrm{e}-006 & 0.4477 \\ \text { Silicon } & 0.0004520 & 0.0004520 & 12.69 \\ \text { Sodium } & 0.0008818 & 0.0008818 & 20.27 \\ \text { Sulfur } & 0.0001393 & 0.0001393 & 4.467\end{array}$




\section{Sample D1019MMTOP}

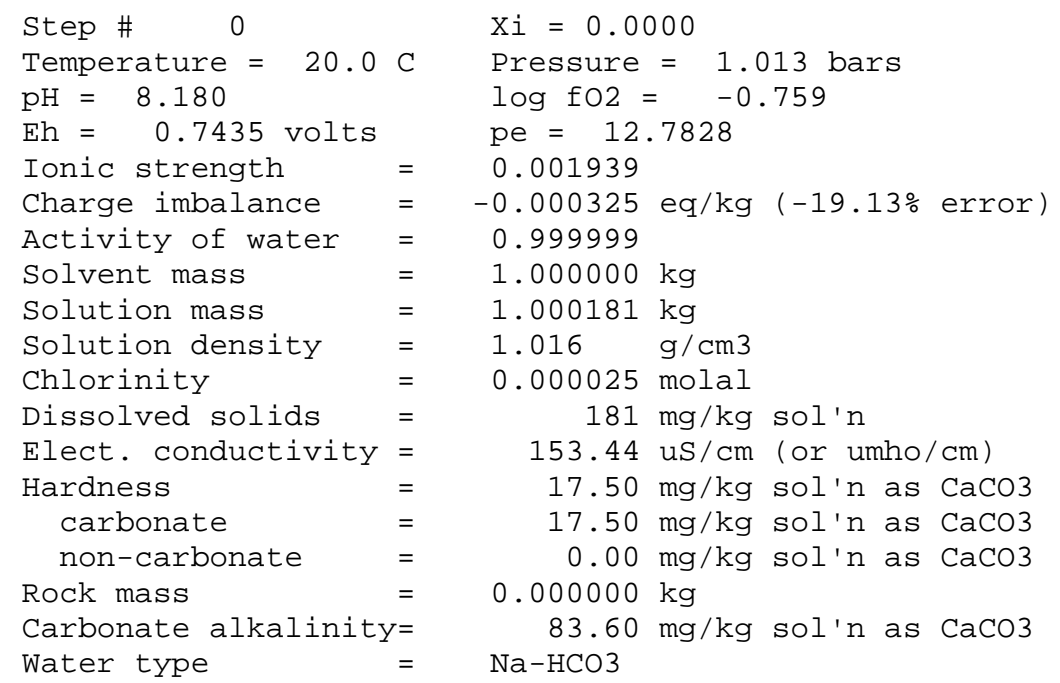

No minerals in system.

\begin{tabular}{|c|c|c|c|c|}
\hline Aqueous species & molality & $\mathrm{mg} / \mathrm{kg}$ sol'n & act. coef. & $\log$ act \\
\hline------ & & ------ & 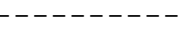 & $--\cdots$ \\
\hline HCO3 - & ๑. .001639 & 100.0 & $\odot .9528$ & -2.8063 \\
\hline $\mathrm{Na}+$ & $\odot .001127$ & 25.91 & $\odot .9528$ & -2.9690 \\
\hline $\operatorname{Si02}(\mathrm{aq})$ & $\odot .0003216$ & 19.32 & 1.0000 & -3.4926 \\
\hline $02(\mathrm{aq})$ & 0.0002442 & 7.812 & 1.0000 & -3.6123 \\
\hline $\mathrm{Ca}++$ & $\odot .0 \odot \odot 1512$ & 6.060 & $\odot .8280$ & -3.9023 \\
\hline $\mathrm{K}+$ & $6.366 e-005$ & 2.488 & 0.9522 & -4.2174 \\
\hline SO4- - & $5.381 e-005$ & 5.168 & 0.8237 & -4.3534 \\
\hline $\mathrm{F}-$ & $3.358 e-005$ & 0.6379 & 0.9525 & -4.4950 \\
\hline $\mathrm{co2}(\mathrm{aq})$ & $2.517 \mathrm{e}-005$ & 1.108 & $1.000 \odot$ & -4.5991 \\
\hline $\mathrm{Cl}-$ & $2.512 \mathrm{e}-\odot \odot 5$ & $\odot .8902$ & $\odot .9522$ & -4.6213 \\
\hline $\mathrm{Mg}++$ & $1.766 \mathrm{e}-005$ & 0.4292 & $\odot .8320$ & -4.8328 \\
\hline NO3- & $1.335 e-005$ & 0.8275 & 0.9522 & -4.8959 \\
\hline Al02- & $1.215 \mathrm{e}-\odot \odot 5$ & $\odot .7165$ & $\odot .9528$ & -4.9364 \\
\hline C03-- & $1.184 \mathrm{e}-005$ & 0.7105 & 0.8248 & -5.0102 \\
\hline $\mathrm{Fe}(\mathrm{OH}) 3(\mathrm{aq})$ & $7.383 e-006$ & 0.7889 & 1.0000 & -5.1318 \\
\hline HSiO3- & $5.007 e-006$ & 0.3859 & $\odot .9528$ & -5.3214 \\
\hline $\mathrm{Zn++}$ & $3.483 e-006$ & $\odot .2277$ & $\odot .8280$ & -5.5401 \\
\hline $\mathrm{NaHCO} 3(\mathrm{aq})$ & $2.646 \mathrm{e}-0 \odot 6$ & $\odot .2222$ & 1..$\odot \odot \odot$ & -5.5774 \\
\hline Re04 - & $2.405 \mathrm{e}-0 \odot 6$ & $\odot .6015$ & $\odot .9528$ & -5.6400 \\
\hline $\mathrm{CaC0} 3(\mathrm{aq})$ & $2.361 \mathrm{e}-006$ & 0.2363 & 1.0000 & -5.6269 \\
\hline $\mathrm{CaHCO} 3+$ & $2.331 \mathrm{e}-006$ & $\odot .2356$ & $\odot .9528$ & -5.6535 \\
\hline $\mathrm{OH}-$ & $1.065 \mathrm{e}-006$ & 0.01810 & $\odot .9525$ & -5.9940 \\
\hline CaSo4(aq) & $7.016 e-0 \odot 7$ & ๑. 09550 & 1. $00 \odot \odot$ & -6.1539 \\
\hline HAl02 (aq) & $2.835 \mathrm{e}-\odot \odot 7$ & ๑. 01701 & $1.00 \odot \odot$ & -6.5474 \\
\hline $\mathrm{MgHCO} 3+$ & $2.671 \mathrm{e}-0 \odot 7$ & 0.02279 & 0.9528 & -6.5943 \\
\hline NaHSiO3 (aq) & $2.559 \mathrm{e}-0 \odot 7$ & ๑. 02560 & $1.000 \odot$ & -6.5920 \\
\hline $\operatorname{MgSO} 4(\mathrm{aq})$ & $1.483 e-0 \odot 7$ & 0.01785 & 1.0000 & -6.8289 \\
\hline $\operatorname{MgC0} 3(\mathrm{aq})$ & 1.309e-007 & 0.01103 & 1.0000 & -6.8831 \\
\hline Mo04 - - & 1. $036 \mathrm{e}-0 \odot 7$ & ๑. 01657 & $\odot .8248$ & -7.0683 \\
\hline $\mathrm{Ba}++$ & $7.286 e-0 \odot 8$ & 0.01000 & 0.8259 & -7.2206 \\
\hline NaC03- & $4.156 e-\odot \odot 8$ & ๑. 003449 & $\odot .9528$ & -7.4023 \\
\hline Znso4(aq) & $2.366 \mathrm{e}-\odot \odot 8$ & ๑. .0०3819 & $1.00 \odot \odot$ & -7.6261 \\
\hline KSO4 - & $2.143 e-0 \odot 8$ & $\odot .0 \odot 2896$ & $\odot .9528$ & -7.6900 \\
\hline $\mathrm{CaF}+$ & 1. $990 \mathrm{e}-\odot \odot 8$ & ๑. . 001176 & $\odot .9528$ & -7.7221 \\
\hline $\mathrm{MgF}+$ & $1.128 \mathrm{e}-008$ & ๑. . $\odot \odot 4882$ & 0.9528 & -7.9688 \\
\hline
\end{tabular}




\begin{tabular}{|c|c|c|c|}
\hline & $\log Q / K$ & & $\log Q / K$ \\
\hline & & & - \\
\hline Nontronite-Ca & $17.9747 \mathrm{~s} / \mathrm{sat}$ & Magnetite & $1.6077 \mathrm{~s} / \mathrm{sat}$ \\
\hline Nontronite-Mg & $17.8499 \mathrm{~s} / \mathrm{sat}$ & Natrolite & $1.5934 \mathrm{~s} / \mathrm{sat}$ \\
\hline Nontronite-Na & $17.6029 \mathrm{~s} / \mathrm{sat}$ & Corundum & $1.5524 \mathrm{~s} / \mathrm{sat}$ \\
\hline Nontronite-K & $17.5448 \mathrm{~s} / \mathrm{sat}$ & Smectite-low-Fe- & $1.4504 \mathrm{~s} / \mathrm{sat}$ \\
\hline Nontronite-H & $16.9004 \mathrm{~s} / \mathrm{sat}$ & Prehnite & $1.2981 \mathrm{~s} / \mathrm{sat}$ \\
\hline Clinoptilolite-h & $13.8366 \mathrm{~s} / \mathrm{sat}$ & Witherite & $1.2039 \mathrm{~s} / \mathrm{sat}$ \\
\hline Clinoptilolite-C & $13.8076 \mathrm{~s} / \mathrm{sat}$ & Albite_high & $0.8773 \mathrm{~s} / \mathrm{sat}$ \\
\hline Hematite & $13.2450 \mathrm{~s} / \mathrm{sat}$ & Clinozoisite & $0.8498 \mathrm{~s} / \mathrm{sat}$ \\
\hline Ferrite-Zn & $12.1440 \mathrm{~s} / \mathrm{sat}$ & Kyanite & $0.8069 \mathrm{~s} / \mathrm{sat}$ \\
\hline Stilbite & $11.0165 \mathrm{~s} / \mathrm{sat}$ & Zoisite & $0.8038 \mathrm{~s} / \mathrm{sat}$ \\
\hline Clinoptilolite-h & $10.6409 \mathrm{~s} / \mathrm{sat}$ & Quartz & $0.6392 \mathrm{~s} / \mathrm{sat}$ \\
\hline Clinoptilolite-N & $10.6401 \mathrm{~s} / \mathrm{sat}$ & Andalusite & $0.5233 \mathrm{~s} / \mathrm{sat}$ \\
\hline Clinoptilolite-K & $10.3244 \mathrm{~s} / \mathrm{sat}$ & Wairakite & $0.4593 \mathrm{~s} / \mathrm{sat}$ \\
\hline Muscovite & $10.2220 \mathrm{~s} / \mathrm{sat}$ & Tridymite & $0.4416 \mathrm{~s} / \mathrm{sat}$ \\
\hline Clinoptilolite-h & $10.2146 \mathrm{~s} / \mathrm{sat}$ & Saponite-Ca & $0.4232 \mathrm{~s} / \mathrm{sat}$ \\
\hline Epidote-ord & $7.9458 \mathrm{~s} / \mathrm{sat}$ & Saponite-Mg & $0.2989 \mathrm{~s} / \mathrm{sat}$ \\
\hline Epidote & $7.9454 \mathrm{~s} / \mathrm{sat}$ & Sillimanite & $0.1499 \mathrm{~s} / \mathrm{sat}$ \\
\hline Mesolite & $7.6652 \mathrm{~s} / \mathrm{sat}$ & Phlogopite & $0.1036 \mathrm{~s} / \mathrm{sat}$ \\
\hline Paragonite & $7.4355 \mathrm{~s} / \mathrm{sat}$ & Cristobalite(alp & $0.0772 \mathrm{~s} / \mathrm{sat}$ \\
\hline Illite & $7.3399 \mathrm{~s} / \mathrm{sat}$ & Chalcedony & $0.0633 \mathrm{~s} / \mathrm{sat}$ \\
\hline Beidellite-Ca & $7.3198 \mathrm{~s} / \mathrm{sat}$ & Saponite-Na & $0.0514 \mathrm{~s} / \mathrm{sat}$ \\
\hline Beidellite-Mg & $7.1949 \mathrm{~s} / \mathrm{sat}$ & Saponite-K & -0.0067 \\
\hline Herschelite & $7.0957 \mathrm{~s} / \mathrm{sat}$ & Dawsonite & -0.0297 \\
\hline Beidellite-Na & $6.9480 \mathrm{~s} / \mathrm{sat}$ & Jadeite & -0.0335 \\
\hline Beidellite-K & $6.8899 \mathrm{~s} / \mathrm{sat}$ & Ice & -0.1194 \\
\hline Andradite & $6.4863 \mathrm{~s} / \mathrm{sat}$ & Coesite & -0.1840 \\
\hline Montmor-Ca & $6.4553 \mathrm{~s} / \mathrm{sat}$ & Cristobalite (bet & -0.3808 \\
\hline Kaolinite & $6.4495 \mathrm{~s} / \mathrm{sat}$ & Kalsilite & -0.4039 \\
\hline Montmor-Mg & $6.4019 \mathrm{~s} / \mathrm{sat}$ & Calcite & $-\odot .4509$ \\
\hline Beidellite-H & $6.2456 \mathrm{~s} / \mathrm{sat}$ & Aragonite & -0.5954 \\
\hline Scolecite & $6.2175 \mathrm{~s} / \mathrm{sat}$ & Saponite-H & -0.6511 \\
\hline Montmor - Na & $6.1514 \mathrm{~s} / \mathrm{sat}$ & Zincite & -0.6585 \\
\hline Goethite & $6.1479 \mathrm{~s} / \mathrm{sat}$ & Dolomite & -0.6732 \\
\hline Montmor-K & 6. $0973 \mathrm{~s} / \mathrm{sat}$ & Dolomite-ord & -0.6732 \\
\hline Pyrophyllite & $6.0517 \mathrm{~s} / \mathrm{sat}$ & $\mathrm{SiO} 2(\mathrm{am})$ & -0.6889 \\
\hline Laumontite & $5.0298 \mathrm{~s} / \mathrm{sat}$ & Analcime-K & -0.7167 \\
\hline Celadonite & $4.2856 \mathrm{~s} / \mathrm{sat}$ & Smithsonite & -0.7172 \\
\hline Margarite & $4.1692 \mathrm{~s} / \mathrm{sat}$ & Talc & -0.8480 \\
\hline Maximum_Microcli & $4.1067 \mathrm{~s} / \mathrm{sat}$ & $\mathrm{Zn}(\mathrm{OH}) 2($ gamma $)$ & -1.0633 \\
\hline K-Feldspar & $4.1055 \mathrm{~s} / \mathrm{sat}$ & $\mathrm{Zn}(\mathrm{OH}) 2$ (epsilon) & -1.0886 \\
\hline Zn2SiO4 & $3.9322 \mathrm{~s} / \mathrm{sat}$ & Clinochlore-14A & -1.2014 \\
\hline Ferrite-Ca & $3.8771 \mathrm{~s} / \mathrm{sat}$ & Monohydrocalcite & -1.2716 \\
\hline Ferrite-Mg & $3.3689 \mathrm{~s} / \mathrm{sat}$ & Anorthite & -1.3194 \\
\hline Lawsonite & $3.2217 \mathrm{~s} / \mathrm{sat}$ & $\mathrm{Zn}(\mathrm{OH}) 2($ beta $)$ & -1.3645 \\
\hline Mordenite & $2.9262 \mathrm{~s} / \mathrm{sat}$ & Smectite-high-Fe & -1.4845 \\
\hline Sanidine_high & $2.8736 \mathrm{~s} / \mathrm{sat}$ & Barite & -1.5075 \\
\hline Diaspore & $2.8257 \mathrm{~s} / \mathrm{sat}$ & Analcime & -1.5183 \\
\hline Amesite-14A & $2.5935 \mathrm{~s} / \mathrm{sat}$ & Magnesite & -1.8823 \\
\hline Boehmite & $2.4132 \mathrm{~s} / \mathrm{sat}$ & Nepheline & -2.1389 \\
\hline Gibbsite & $2.2537 \mathrm{~s} / \mathrm{sat}$ & Dolomite-dis & -2.2550 \\
\hline Albite_low & $2.2297 \mathrm{~s} / \mathrm{sat}$ & $\mathrm{Fe}(\mathrm{OH}) 3$ & -2.5399 \\
\hline Albite & $2.2296 \mathrm{~s} / \mathrm{sat}$ & Fluorite & -2.8054 \\
\hline (only minerals & vith $\log Q / K>$ & -3 listed) & \\
\hline Gases & fugacity & $\log$ fug. & \\
\hline - - - - - - - - - - - - - & $-\ldots-\ldots$ & 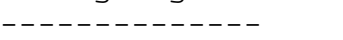 & \\
\hline $02(g)$ & 0.1742 & -0.759 & \\
\hline $\mathrm{H} 2 \mathrm{O}(\mathrm{g})$ & 0.01903 & -1.721 & \\
\hline $\operatorname{co2}(\mathrm{g})$ & 0.0006295 & -3.201 & \\
\hline $\mathrm{HF}(\mathrm{g})$ & $1.068 e-014$ & -13.971 & \\
\hline
\end{tabular}




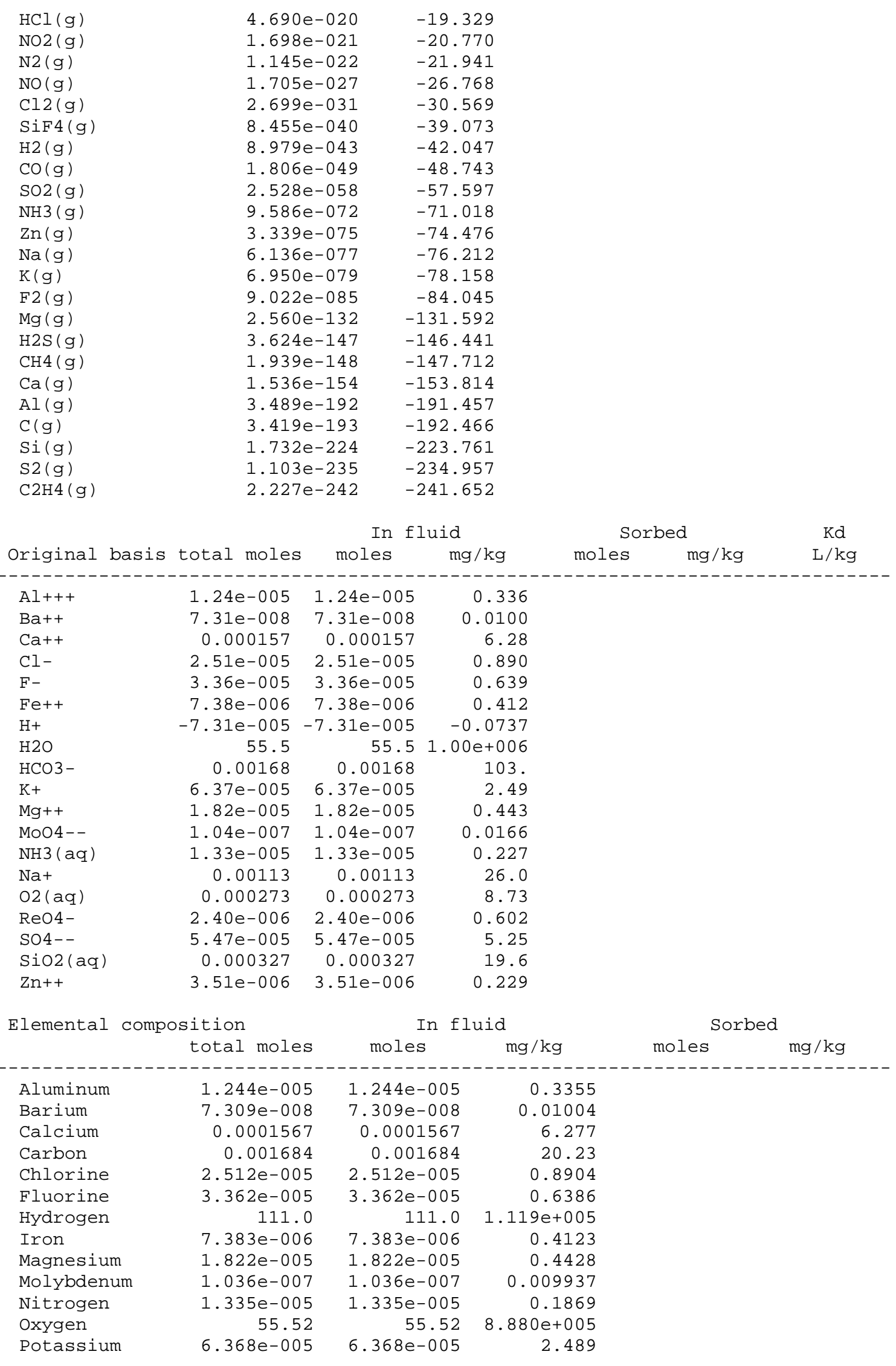




$\begin{array}{lrrr}\text { Rhenium } & 2.405 \mathrm{e}-006 & 2.405 \mathrm{e}-006 & 0.4477 \\ \text { Silicon } & 0.0003269 & 0.0003269 & 9.180 \\ \text { Sodium } & 0.001130 & 0.001130 & 25.97 \\ \text { Sulfur } & 5.470 \mathrm{e}-005 & 5.470 \mathrm{e}-005 & 1.754 \\ \text { Zinc } & 3.507 \mathrm{e}-006 & 3.507 \mathrm{e}-006 & 0.2292\end{array}$




\section{Sample D10NBTW}

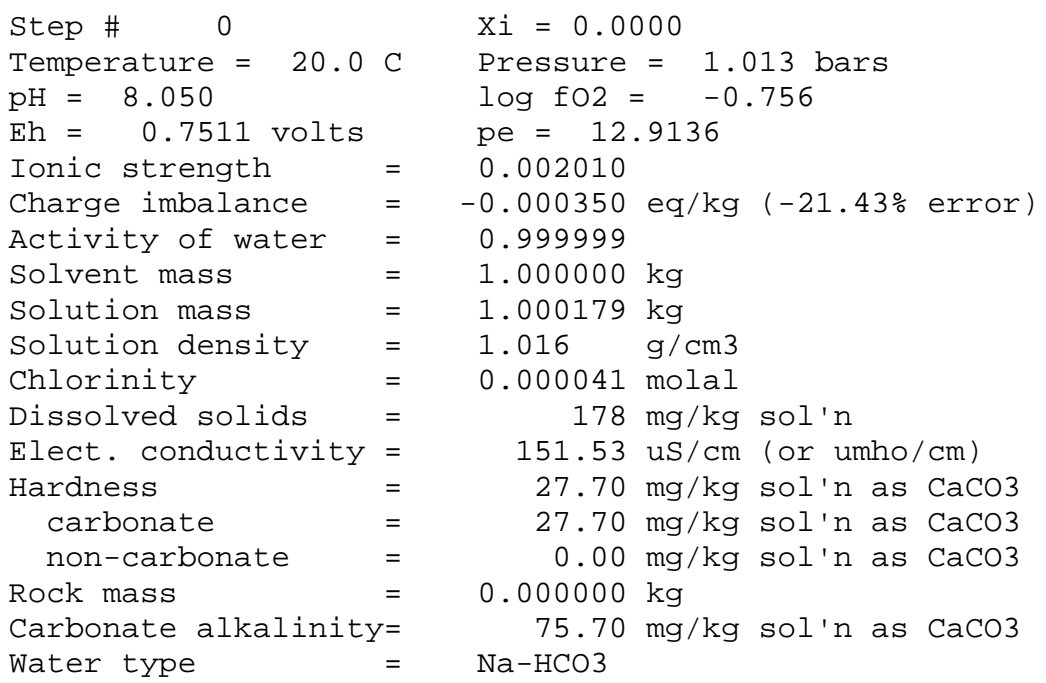

No minerals in system.

\begin{tabular}{|c|c|c|c|c|}
\hline Aqueous species & molality & $\mathrm{mg} / \mathrm{kg}$ sol'n & act. coef. & $\log$ act \\
\hline - - - - - - - - - - - - & ------ & 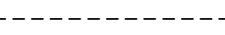 & 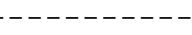 & ---- \\
\hline HCO3 - & $\odot .0 \odot 1489$ & 90.84 & $\odot .9520$ & -2.8484 \\
\hline $\mathrm{Na}+$ & ๑. . ๑९८327 & 19.14 & $\odot .9520$ & -3.1009 \\
\hline $\operatorname{Si02}(\mathrm{aq})$ & ๑. . 0004224 & 25.38 & 1.0000 & -3.3743 \\
\hline $02(\mathrm{aq})$ & 0.0002460 & 7.871 & 1.0000 & -3.6090 \\
\hline $\mathrm{Ca}++$ & $\odot .0002056$ & 8.238 & 0.8254 & -3.7704 \\
\hline SO4- - & $9.964 \mathrm{e}-\odot \odot 5$ & 9.570 & 0.8210 & -4.0872 \\
\hline $\mathrm{K}+$ & $7.923 e-\odot \odot 5$ & 3.097 & $\odot .9514$ & -4.1227 \\
\hline $\mathrm{Mg}++$ & $6.221 e-005$ & 1.512 & 0.8296 & -4.2873 \\
\hline Cl- & $4.135 e-005$ & 1.466 & $\odot .9514$ & -4.4052 \\
\hline $\mathrm{F}-$ & $3.810 \mathrm{e}-005$ & $\odot .7236$ & 0.9517 & -4.4406 \\
\hline $\mathrm{co2}(\mathrm{aq})$ & $3.082 e-005$ & 1.356 & 1.0000 & -4.5112 \\
\hline Alo2 - & 1. $481 \mathrm{e}-\odot \odot 5$ & $\odot .8733$ & $\odot .9520$ & -4.8508 \\
\hline CO3-- & $7.993 e-006$ & 0.4796 & $\odot .8221$ & -5.1824 \\
\hline HSi03- & $4.878 e-\odot \odot 6$ & 0.3760 & 0.9520 & -5.3331 \\
\hline CaHCO3+ & $2.869 \mathrm{e}-\odot \odot 6$ & $\odot .2900$ & $\odot .9520$ & -5.5636 \\
\hline $\mathrm{Zn++}$ & $2.809 \mathrm{e}-006$ & 0.1837 & $\odot .8254$ & -5.6348 \\
\hline Re04 - & $2.405 e-006$ & 0.6015 & 0.9520 & -5.6403 \\
\hline Cac03(aq) & $2.152 \mathrm{e}-006$ & 0.2154 & 1.0000 & -5.6671 \\
\hline $\operatorname{NaHCO} 3(\mathrm{aq})$ & $1.773 \mathrm{e}-006$ & 0.1489 & 1.0000 & -5.7514 \\
\hline CaSO4(aq) & 1. $755 \mathrm{e}-\odot \odot 6$ & 0.2388 & 1.0000 & -5.7558 \\
\hline $\operatorname{MgSO} 4(\mathrm{aq})$ & $9.610 \mathrm{e}-007$ & 0.1157 & 1.0000 & -6.0173 \\
\hline $\mathrm{MgHCO} 3+$ & $8.520 \mathrm{e}-0 \odot 7$ & ๑.07268 & 0.9520 & -6.0909 \\
\hline $\mathrm{OH}-$ & $7.898 \mathrm{e}-\odot \odot 7$ & 0.01343 & 0.9517 & -6.1240 \\
\hline HAl02 (aq) & $4.659 \mathrm{e}-\odot \odot 7$ & ๑. . 02794 & 1.0000 & -6.3317 \\
\hline $\operatorname{MgC0} 3(\mathrm{aq})$ & 3. $.93 e-\odot \odot 7$ & 0.02607 & $1.000 \odot$ & -6.5097 \\
\hline NaHSiO3(aq) & $1.839 \mathrm{e}-007$ & $\odot .0184 \odot$ & 1.0000 & -6.7355 \\
\hline $\mathrm{Ba}++$ & $6.721 \mathrm{e}-008$ & ๑. . 009228 & 0.8232 & -7.2570 \\
\hline Mo04-- & $5.949 \mathrm{e}-\odot \odot 8$ & ๑ . ๑๑9513 & $\odot .8221$ & -7.3106 \\
\hline KSO4 - & $4.922 \mathrm{e}-\odot \odot 8$ & ๑. .๑66652 & $\odot .9520$ & -7.3292 \\
\hline MgF+ & $4.492 \mathrm{e}-008$ & $\odot .0 \odot 1945$ & $\odot .9520$ & -7.3689 \\
\hline Znso4( aq) & $3.510 \mathrm{e}-008$ & 0.005667 & 1.0000 & -7.4546 \\
\hline $\mathrm{CaF}+$ & $3.06 \odot \mathrm{e}-0 \odot 8$ & $\odot .001807$ & 0.9520 & -7.5357 \\
\hline $\mathrm{NaCO3-}$ & $2.066 \mathrm{e}-008$ & $\odot .001714$ & $\odot .9520$ & -7.7063 \\
\hline
\end{tabular}




\begin{tabular}{|c|c|c|c|}
\hline & $\log Q / K$ & & $\log Q / K$ \\
\hline & & & -------- \\
\hline Stilbite & $12.1274 \mathrm{~s} / \mathrm{sat}$ & Saponite-K & $1.3437 \mathrm{~s} / \mathrm{sat}$ \\
\hline Muscovite & $11.1887 \mathrm{~s} / \mathrm{sat}$ & Saponite-Na & $1.3271 \mathrm{~s} / \mathrm{sat}$ \\
\hline Illite & $8.3003 \mathrm{~s} / \mathrm{sat}$ & Wairakite & $1.2359 \mathrm{~s} / \mathrm{sat}$ \\
\hline Beidellite-Ca & $8.2355 \mathrm{~s} / \mathrm{sat}$ & Albite_high & $1.1862 \mathrm{~s} / \mathrm{sat}$ \\
\hline Mesolite & $8.1894 \mathrm{~s} / \mathrm{sat}$ & Andalusite & $1.0730 \mathrm{~s} / \mathrm{sat}$ \\
\hline Beidellite-Mg & $8.1788 \mathrm{~s} / \mathrm{sat}$ & Clinochlore-14A & 1. $0125 \mathrm{~s} / \mathrm{sat}$ \\
\hline Paragonite & $8.1756 \mathrm{~s} / \mathrm{sat}$ & Witherite & $0.9954 \mathrm{~s} / \mathrm{sat}$ \\
\hline Beidellite-K & $7.8151 \mathrm{~s} / \mathrm{sat}$ & Quartz & $0.7576 \mathrm{~s} / \mathrm{sat}$ \\
\hline Beidellite-Na & $7.7983 \mathrm{~s} / \mathrm{sat}$ & Saponite-H & $0.7110 \mathrm{~s} / \mathrm{sat}$ \\
\hline Herschelite & $7.6147 \mathrm{~s} / \mathrm{sat}$ & Sillimanite & $0.6995 \mathrm{~s} / \mathrm{sat}$ \\
\hline Montmor-Mg & $7.3767 \mathrm{~s} / \mathrm{sat}$ & Tridymite & $0.5599 \mathrm{~s} / \mathrm{sat}$ \\
\hline Montmor-Ca & $7.3619 \mathrm{~s} / \mathrm{sat}$ & Talc & $0.4820 \mathrm{~s} / \mathrm{sat}$ \\
\hline Beidellite-H & $7.1823 \mathrm{~s} / \mathrm{sat}$ & Cristobalite(alp & $0.1955 \mathrm{~s} / \mathrm{sat}$ \\
\hline Kaolinite & $7.1175 \mathrm{~s} / \mathrm{sat}$ & Chalcedony & $0.1816 \mathrm{~s} / \mathrm{sat}$ \\
\hline Montmor-K & $7.0134 \mathrm{~s} / \mathrm{sat}$ & Jadeite & $0.1570 \mathrm{~s} / \mathrm{sat}$ \\
\hline Montmor-Na & $6.9927 \mathrm{~s} / \mathrm{sat}$ & Dawsonite & $0.0120 \mathrm{~s} / \mathrm{sat}$ \\
\hline Pyrophyllite & $6.9564 \mathrm{~s} / \mathrm{sat}$ & Coesite & -0.0657 \\
\hline Scolecite & $6.8758 \mathrm{~s} / \mathrm{sat}$ & Kalsilite & -0.1053 \\
\hline Laumontite & $5.8064 \mathrm{~s} / \mathrm{sat}$ & Ice & -0.1194 \\
\hline Celadonite & $5.2249 \mathrm{~s} / \mathrm{sat}$ & Cristobalite (bet & -0.2625 \\
\hline Margarite & $5.1404 \mathrm{~s} / \mathrm{sat}$ & Analcime-K & -0.3022 \\
\hline Amesite-14A & $4.8348 \mathrm{~s} / \mathrm{sat}$ & Dolomite & $-\odot .340 \odot$ \\
\hline Maximum_Microcli & $4.6421 \mathrm{~s} / \mathrm{sat}$ & Dolomite-ord & -0.3400 \\
\hline K-Feldspar & $4.6409 \mathrm{~s} / \mathrm{sat}$ & Calcite & -0.4911 \\
\hline Lawsonite & $3.7616 \mathrm{~s} / \mathrm{sat}$ & $\mathrm{Si02}(\mathrm{am})$ & -0.5706 \\
\hline Mordenite & $3.5961 \mathrm{~s} / \mathrm{sat}$ & Aragonite & -0.6356 \\
\hline Sanidine_high & $3.4090 \mathrm{~s} / \mathrm{sat}$ & Anorthite & -0.7794 \\
\hline Zn2SiO4 & $3.3411 \mathrm{~s} / \mathrm{sat}$ & Smithsonite & -0.9840 \\
\hline Diaspore & $3.0413 \mathrm{~s} / \mathrm{sat}$ & Zincite & -1.0133 \\
\hline Boehmite & $2.6289 \mathrm{~s} / \mathrm{sat}$ & Barite & -1.2778 \\
\hline Albite_low & $2.5385 \mathrm{~s} / \mathrm{sat}$ & Monohydrocalcite & -1.3118 \\
\hline Albite & $2.5384 \mathrm{~s} / \mathrm{sat}$ & Analcime & -1.3212 \\
\hline Gibbsite & $2.4693 \mathrm{~s} / \mathrm{sat}$ & $\mathrm{Zn}(\mathrm{OH}) 2($ gamma $)$ & -1.4180 \\
\hline Corundum & $1.9837 \mathrm{~s} / \mathrm{sat}$ & $\mathrm{Zn}(\mathrm{OH}) 2$ (epsilon) & -1.4433 \\
\hline Natrolite & $1.8561 \mathrm{~s} / \mathrm{sat}$ & Magnesite & -1.5090 \\
\hline Prehnite & $1.8284 \mathrm{~s} / \mathrm{sat}$ & $\mathrm{Zn}(\mathrm{OH}) 2($ beta $)$ & -1.7192 \\
\hline Saponite-Ca & $1.7641 \mathrm{~s} / \mathrm{sat}$ & Dolomite-dis & -1.9218 \\
\hline Saponite-Mg & $1.7081 \mathrm{~s} / \mathrm{sat}$ & Nepheline & -2.0667 \\
\hline Clinozoisite & $1.5956 \mathrm{~s} / \mathrm{sat}$ & Clinochlore-7A & -2.4024 \\
\hline Zoisite & $1.5497 \mathrm{~s} / \mathrm{sat}$ & Fluorite & -2.5647 \\
\hline Phlogopite & $1.4955 \mathrm{~s} / \mathrm{sat}$ & Cordierite_hydr & -2.6095 \\
\hline Kyanite & $1.3565 \mathrm{~s} / \mathrm{sat}$ & & \\
\hline (only minerals & Iith $\log Q / K>$ & 3 listed) & \\
\hline Gases & fugacity & $\log$ fug. & \\
\hline 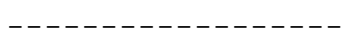 & - - - - - & - - - - - - & \\
\hline $02(g)$ & 0.1755 & $-\odot .756$ & \\
\hline $\mathrm{H} 2 \mathrm{O}(\mathrm{g})$ & 0.01903 & -1.721 & \\
\hline $\operatorname{co2}(\mathrm{g})$ & $\odot .0007707$ & -3.113 & \\
\hline$H F(g)$ & $1.634 \mathrm{e}-014$ & -13.787 & \\
\hline $\mathrm{HCl}(\mathrm{g})$ & 1. $041 e-019$ & -18.983 & \\
\hline $\operatorname{cl} 2(\mathrm{~g})$ & $1.334 \mathrm{e}-030$ & -29.875 & \\
\hline $\operatorname{SiF} 4(g)$ & $6.069 e-039$ & -38.217 & \\
\hline $\mathrm{H} 2(\mathrm{~g})$ & $8.946 e-043$ & -42.048 & \\
\hline $\operatorname{co}(\mathrm{g})$ & $2.203 e-\odot 49$ & -48.657 & \\
\hline So2(g) & $8.458 e-058$ & -57.073 & \\
\hline $\mathrm{Zn}(\mathrm{g})$ & $1.470 \mathrm{e}-075$ & -74.833 & \\
\hline $\mathrm{Na}(\mathrm{g})$ & $3.351 \mathrm{e}-077$ & -76.475 & \\
\hline $\mathrm{K}(\mathrm{g})$ & $6.395 e-079$ & -78.194 & \\
\hline $\mathrm{F} 2(\mathrm{~g})$ & $2.117 \mathrm{e}-084$ & -83.674 & \\
\hline
\end{tabular}




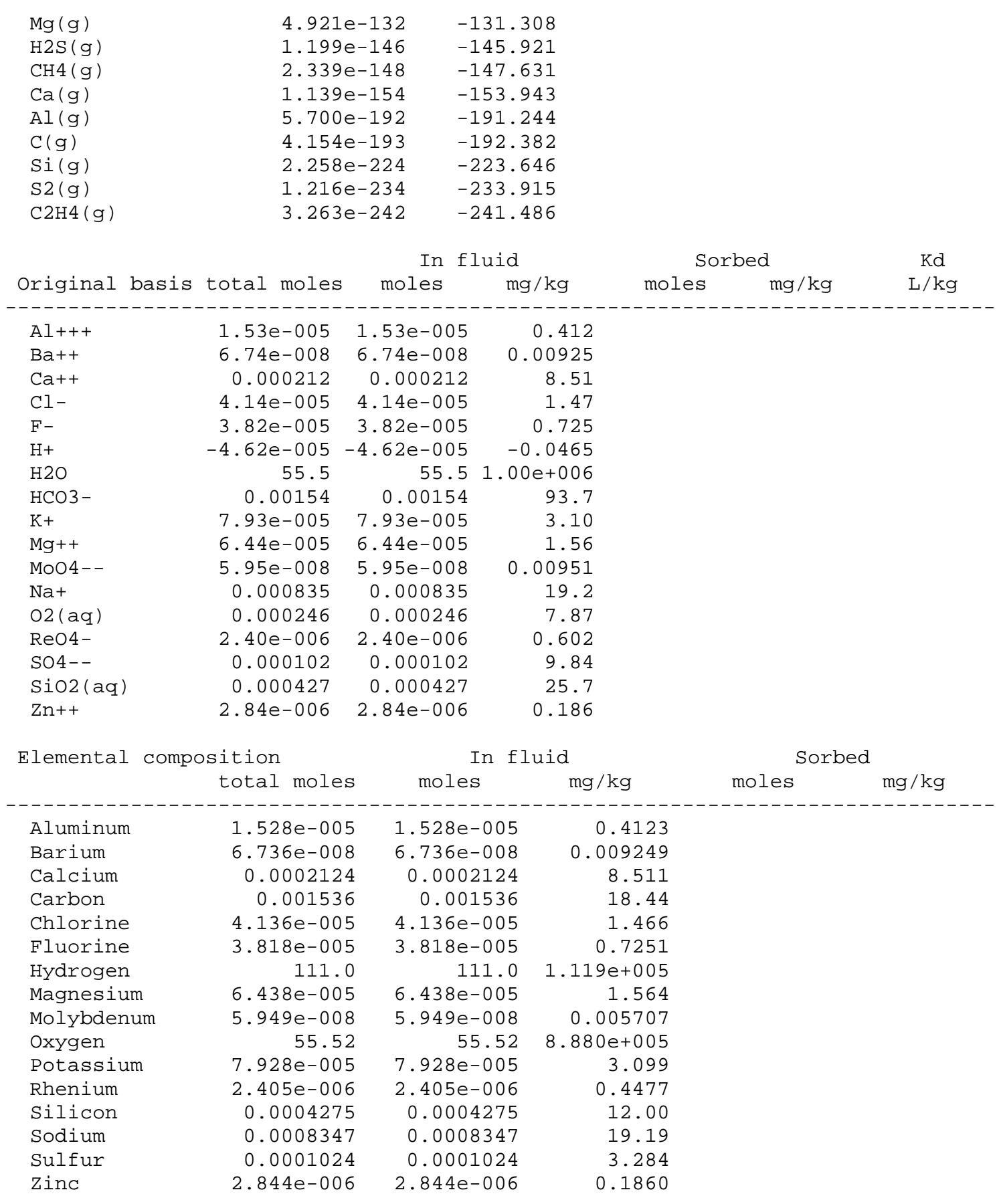




\section{Sample D10NB0T}

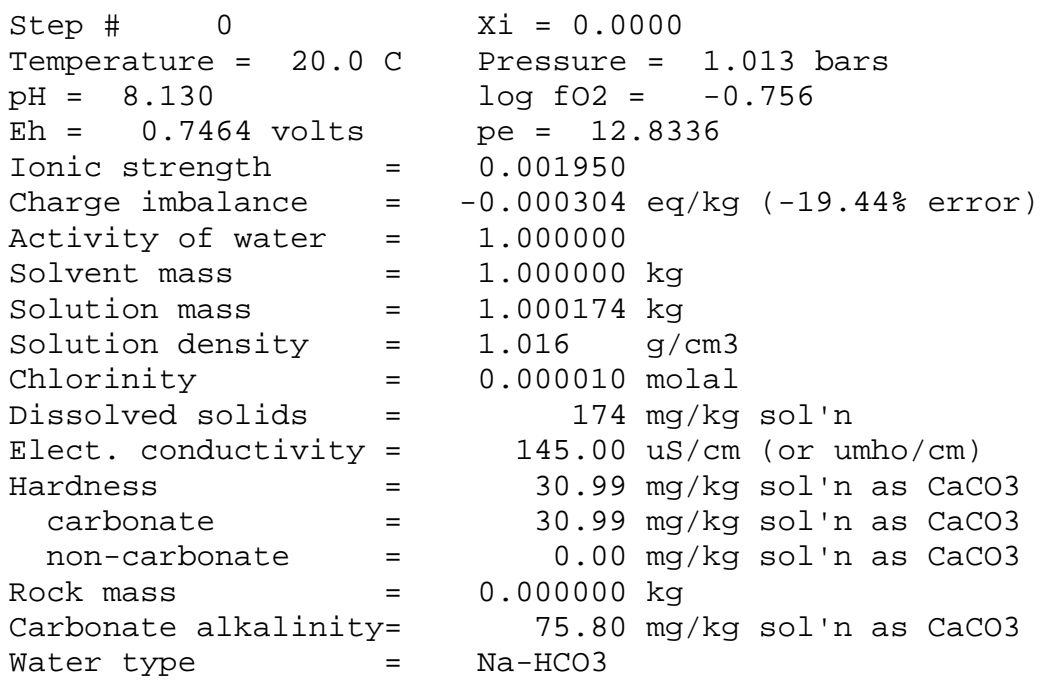

No minerals in system.

\begin{tabular}{|c|c|c|c|c|}
\hline Aqueous species & molality & $\mathrm{mg} / \mathrm{kg}$ sol'n & act. coef. & $\log$ act \\
\hline.---- & & & $-\ldots-\ldots$ & $---{ }_{-1}$ \\
\hline $\mathrm{HCO}-$ & $\odot .001487$ & $9 \odot .7 \odot$ & $\odot .9527$ & -2.8488 \\
\hline $\mathrm{Na}+$ & $\odot .0 \odot \odot 7216$ & 16.59 & $\odot .9527$ & -3.1627 \\
\hline $\operatorname{Si02}(\mathrm{aq})$ & $\odot .0004318$ & 25.94 & 1.0000 & -3.3647 \\
\hline $02(\mathrm{aq})$ & $\odot .0 \odot \odot 2460$ & 7.871 & 1.0000 & -3.6090 \\
\hline $\mathrm{Ca}++$ & $\odot .0 \odot \odot 2332$ & 9.345 & $\odot .8276$ & -3.7145 \\
\hline $\mathrm{K}+$ & $8.252 e-005$ & 3.226 & 0.9520 & -4.1048 \\
\hline SO4 - - & $7.181 \mathrm{e}-0 \odot 5$ & 6.898 & 0.8233 & -4.2282 \\
\hline $\mathrm{Mg}++$ & $6.672 e-0 \odot 5$ & 1.621 & 0.8317 & -4.2558 \\
\hline $\mathrm{F}-$ & $3.540 \mathrm{e}-005$ & 0.6725 & 0.9524 & -4.4722 \\
\hline $\mathrm{co2}(\mathrm{aq})$ & $2.561 \mathrm{e}-\odot \odot 5$ & 1.127 & $1.000 \odot$ & -4.5916 \\
\hline NO3- & $1.300 \mathrm{e}-005$ & 0.8058 & 0.9520 & -4.9075 \\
\hline Cl - & 1. $041 \mathrm{e}-005$ & 0.3689 & 0.9520 & $-5.0 \odot 4 \odot$ \\
\hline CO3-- & $9.575 e-\odot \odot 6$ & 0.5745 & 0.8244 & -5.1027 \\
\hline HSi03 - & 5. $991 \mathrm{e}-006$ & $\odot .4618$ & $\odot .9527$ & -5.2435 \\
\hline $\mathrm{CaHCO}+$ & $3.258 e-0 \odot 6$ & 0.3293 & $\odot .9527$ & -5.5081 \\
\hline $\mathrm{CaCO} 3(\mathrm{aq})$ & $2.941 \mathrm{e}-0 \odot 6$ & 0.2943 & $1.0 \odot \odot \odot$ & -5.5316 \\
\hline $\mathrm{Zn}++$ & $2.595 e-006$ & 0.1697 & 0.8276 & -5.6680 \\
\hline Re04 - & $2.405 \mathrm{e}-\odot \odot 6$ & 0.6015 & $\odot .9527$ & -5.6400 \\
\hline $\operatorname{NaHCO} 3(\mathrm{aq})$ & $1.536 \mathrm{e}-\odot \odot 6$ & $\odot .1290$ & $1.00 \odot \odot$ & -5.8136 \\
\hline CaSO4(aq) & 1. $442 \mathrm{e}-006$ & 0.1963 & 1.0000 & -5.8409 \\
\hline $\mathrm{OH}-$ & $9.489 \mathrm{e}-0 \odot 7$ & 0.01614 & $\odot .9524$ & -6.0440 \\
\hline $\mathrm{MgHCO}+$ & $9.147 \mathrm{e}-0 \odot 7$ & 0.07803 & $\odot .9527$ & -6.0598 \\
\hline $\operatorname{MgS0} 4(\mathrm{aq})$ & $7.469 \mathrm{e}-\odot \odot 7$ & ๑. . 08989 & 1.0000 & -6.1267 \\
\hline $\operatorname{MgCO} 3(\mathrm{aq})$ & $3.995 \mathrm{e}-0 \odot 7$ & 0.03368 & 1.0000 & -6.3985 \\
\hline NaHSiO3 (aq) & 1. $960 \mathrm{e}-007$ & ๑. . 01961 & 1.0000 & -6.7078 \\
\hline MoO4 - - & 1. $\odot \odot 5 e-\odot \odot 7$ & ๑. . 01607 & $\odot .8244$ & -7.0816 \\
\hline $\mathrm{Ba}++$ & $7.290 \mathrm{e}-0 \odot 8$ & ๑. . 101001 & $\odot .8255$ & -7.2206 \\
\hline $\mathrm{MgF}+$ & $4.489 e-0 \odot 8$ & 0.001944 & 0.9527 & -7.3689 \\
\hline KSO4 - & $3.705 \mathrm{e}-0 \odot 8$ & 0.005007 & $\odot .9527$ & -7.4522 \\
\hline $\mathrm{CaF}+$ & $3.234 \mathrm{e}-0 \odot 8$ & ๑. 001910 & 0.9527 & -7.5113 \\
\hline $\mathrm{ZnSO4}(\mathrm{aq})$ & 2. $350 \mathrm{e}-\odot \odot 8$ & 0.003794 & 1.0000 & -7.6289 \\
\hline $\mathrm{NaCO} 3-$ & $2.150 \mathrm{e}-\odot \odot 8$ & ๑. . 011785 & $\odot .9527$ & -7.6885 \\
\hline
\end{tabular}




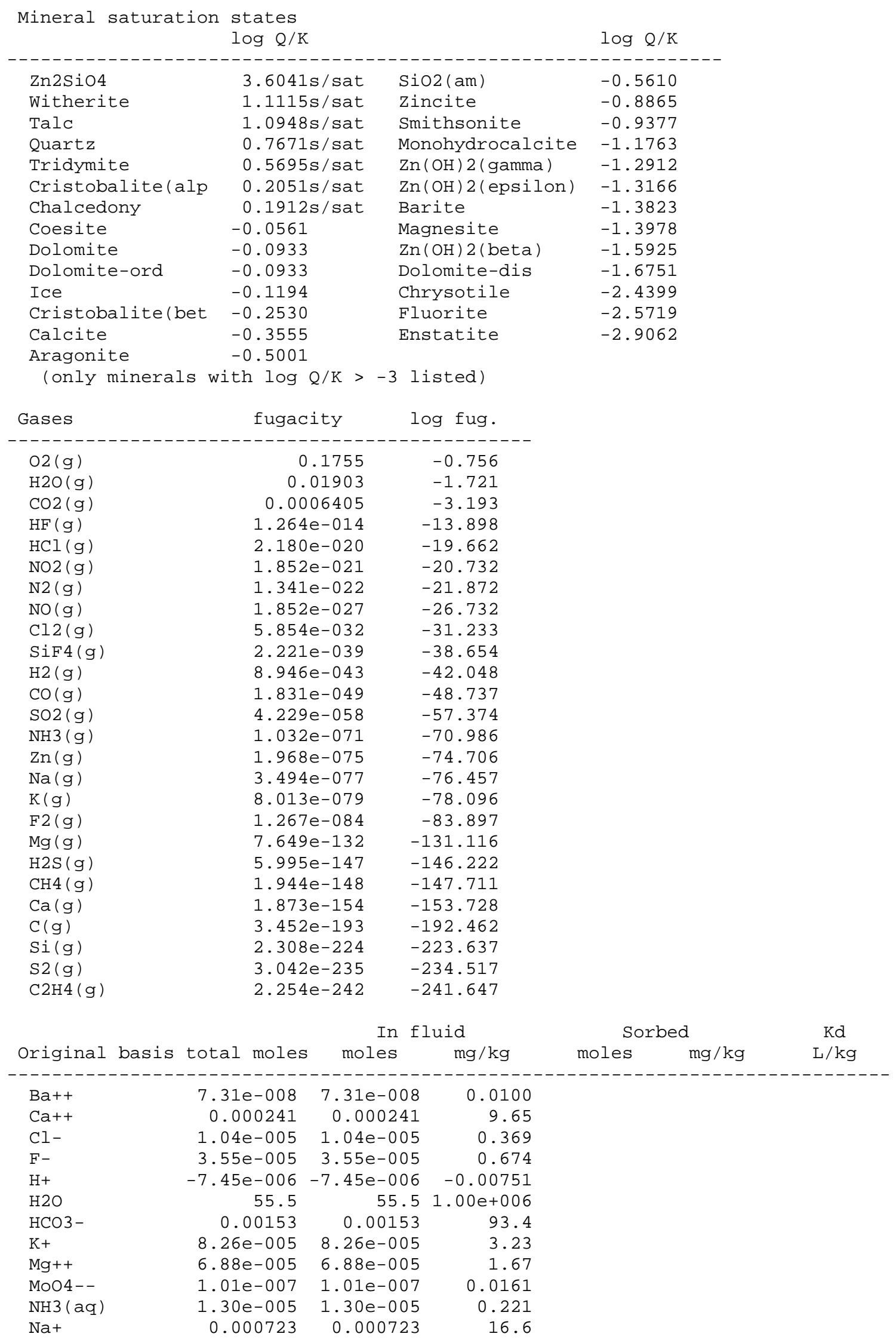




\begin{tabular}{|c|c|c|c|c|}
\hline $02(\mathrm{aq})$ & 0.000272 & 0.000272 & \multicolumn{2}{|l|}{8.70} \\
\hline Re04- & $2.40 \mathrm{e}-006$ & $2.40 e-0 \odot 6$ & \multicolumn{2}{|l|}{ 0. 602} \\
\hline SO4 - - & $7.41 \mathrm{e}-0 \odot 5$ & $7.41 \mathrm{e}-\odot \odot 5$ & \multicolumn{2}{|l|}{7.11} \\
\hline $\operatorname{Si02}(\mathrm{aq})$ & 0.000438 & 0.000438 & \multicolumn{2}{|l|}{$\begin{array}{l}7.11 \\
26.3\end{array}$} \\
\hline $\mathrm{Zn++}$ & $2.62 \mathrm{e}-006$ & $2.62 \mathrm{e}-006$ & \multicolumn{2}{|l|}{. 171} \\
\hline \multicolumn{4}{|c|}{ Elemental composition } & Sorbed \\
\hline & total moles & moles & $\mathrm{mg} / \mathrm{kg}$ & moles $\quad \mathrm{mg} / \mathrm{kg}$ \\
\hline------- & 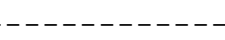 & 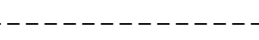 & 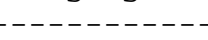 & - \\
\hline Barium & $7.309 e-\odot \odot 8$ & $7.309 e-008$ & 0.01004 & \\
\hline Calcium & $\odot . \odot \odot \odot 24 \odot 9$ & $\odot .0 \odot \odot 2409$ & 9.652 & \\
\hline Carbon & $\odot .001531$ & $\odot .001531$ & 18.39 & \\
\hline Chlorine & $1.041 e-005$ & $1.041 \mathrm{e}-005$ & 0.3690 & \\
\hline Fluorine & $3.548 \mathrm{e}-005$ & $3.548 \mathrm{e}-005$ & 0.6740 & \\
\hline Hydrogen & 111.0 & 111.0 & $1.119 \mathrm{e}+005$ & \\
\hline Magnesium & $6.883 e-0 \odot 5$ & $6.883 e-005$ & 1.673 & \\
\hline Molybdenum & 1. $\odot \odot 5 e-\odot \odot 7$ & 1. $\odot \odot 5 e-\odot \odot 7$ & $\odot .0 \odot 9642$ & \\
\hline Nitrogen & $1.300 \mathrm{e}-005$ & $1.300 \mathrm{e}-005$ & 0.1820 & \\
\hline oxygen & 55.51 & 55.51 & 8. 881e+००5 & \\
\hline Potassium & $8.256 \mathrm{e}-005$ & $8.256 \mathrm{e}-\odot \odot 5$ & 3.227 & \\
\hline Rhenium & $2.405 e-0 \odot 6$ & $2.405 e-006$ & 0.4477 & \\
\hline Silicon & $\odot .00 \odot 438 \odot$ & $\odot .00 \odot 438 \odot$ & 12.30 & \\
\hline Sodium & $\odot .0007234$ & ๑. . 0007234 & 16.63 & \\
\hline Sulfur & 7. 4०6e-๑०5 & 7. 4०6e-๑०5 & 2.375 & \\
\hline Zinc & $2.619 \mathrm{e}-\odot \odot 6$ & $2.619 \mathrm{e}-0 \odot 6$ & 0.1712 & \\
\hline
\end{tabular}




\section{Sample D10EB0T}

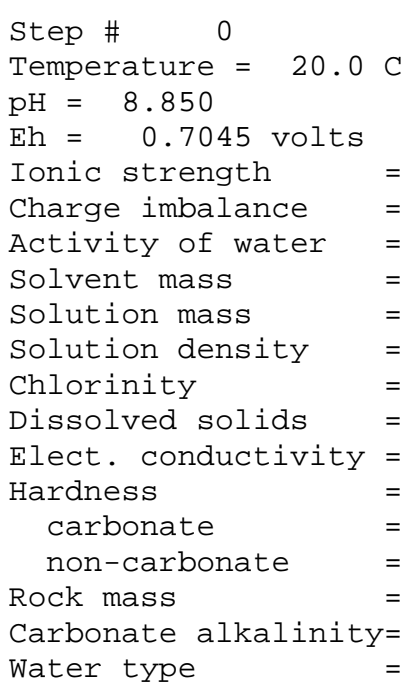

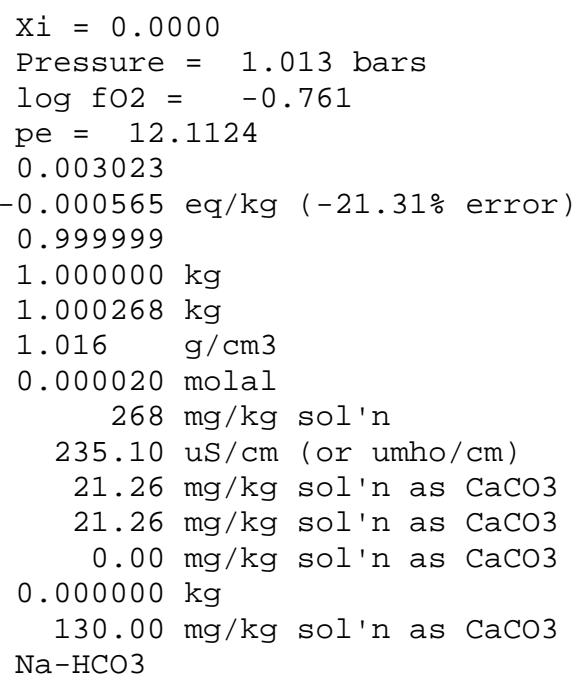

No minerals in system.

\begin{tabular}{|c|c|c|c|c|}
\hline Aqueous species & molality & $\mathrm{mg} / \mathrm{kg}$ sol'n & act. coef. & $\log$ act. \\
\hline$-x_{1}$ & & & ------ & \\
\hline HCO3 - & 0.002401 & 146.4 & 0.9422 & -2.6455 \\
\hline $\mathrm{Na}+$ & ๑ . 001929 & 44.34 & $\odot .9422$ & -2.7404 \\
\hline $\operatorname{Si02}(\mathrm{aq})$ & 0.0004543 & 27.29 & 1.0000 & -3.3427 \\
\hline $02(\mathrm{aq})$ & $\odot .0002433$ & 7.783 & 1.0000 & -3.6139 \\
\hline $\mathrm{Ca}++$ & 0.0001690 & 6.770 & 0.7937 & -3.8725 \\
\hline SO4 - - & $9.312 e-005$ & 8.943 & 0.7876 & -4.1347 \\
\hline CO3-- & $8.384 \mathrm{e}-\odot \odot 5$ & 5.030 & ๑.7891 & -4.1794 \\
\hline $\mathrm{F}-$ & $7.916 e-005$ & 1.504 & $\odot .9418$ & -4.1275 \\
\hline $\mathrm{K}+$ & $6.038 e-\odot \odot 5$ & $2.36 \odot$ & $\odot .9413$ & -4.2454 \\
\hline HSiO3 - & $3.344 \mathrm{e}-0 \odot 5$ & 2.578 & $\odot .9422$ & -4.5015 \\
\hline $\mathrm{Cl}-$ & $2.017 e-005$ & 0.7150 & 0.9413 & -4.7215 \\
\hline $\mathrm{Mg}++$ & 1. $979 \mathrm{e}-\odot \odot 5$ & $\odot .4809$ & $\odot .7995$ & -4.8007 \\
\hline Al02- & 1. $909 \mathrm{e}-005$ & 1.125 & 0.9422 & -4.7451 \\
\hline $\mathrm{CaC03}(\mathrm{aq})$ & $1.713 e-005$ & 1.714 & 1.0000 & -4.7663 \\
\hline N03- & $1.528 \mathrm{e}-0 \odot 5$ & $\odot .9475$ & $\odot .9413$ & -4.8420 \\
\hline $\mathrm{Fe}(\mathrm{OH}) 3(\mathrm{aq})$ & 1. 098 e- $\odot \odot 5$ & 1.173 & $1.00 \odot \odot$ & -4.9595 \\
\hline $\mathrm{co2}(\mathrm{aq})$ & $7.794 \mathrm{e}-006$ & $\odot .3429$ & 1.0000 & -5.1083 \\
\hline $\operatorname{NaHCO3}(\mathrm{aq})$ & $6.486 \mathrm{e}-0 \odot 6$ & $\odot .5447$ & 1.0000 & -5.1880 \\
\hline $\mathrm{OH}-$ & $5.036 e-006$ & ๑. . 08563 & 0.9418 & -5.3240 \\
\hline Re04 - & $4.788 e-006$ & 1.198 & 0.9422 & -5.3457 \\
\hline CaHCO3+ & $3.655 \mathrm{e}-006$ & ๑. 3695 & 0.9422 & -5.4629 \\
\hline NaHSiO3 (aq) & $2.861 \mathrm{e}-\odot \odot 6$ & 0.2863 & $1.000 \odot$ & -5.5435 \\
\hline MoO4- - & $2.544 \mathrm{e}-006$ & 0.4068 & 0.7891 & -5.6973 \\
\hline CaSo4(aq) & 1. $243 e-006$ & 0.1692 & 1.0000 & -5.9055 \\
\hline $\operatorname{MgC0} 3(\mathrm{aq})$ & $9.547 e-007$ & ๑. .08047 & 1.0000 & -6.0201 \\
\hline $\mathrm{NaCO} 3-$ & $4.818 e-0 \odot 7$ & ๑.03998 & 0.9422 & -6.3429 \\
\hline MgHCO3+ & $4.212 e-007$ & ๑. .03593 & 0.9422 & -6.4014 \\
\hline $\operatorname{MgSO} 4(\mathrm{aq})$ & $2.642 \mathrm{e}-0 \odot 7$ & ๑. . 03179 & $1.000 \odot$ & -6.5781 \\
\hline $\mathrm{Ba}++$ & $9.898 e-0 \odot 8$ & ๑. 01359 & 0.7907 & -7.1065 \\
\hline HAl02 (aq) & $9.417 \mathrm{e}-\odot \odot 8$ & ๑. 005648 & $1.00 \odot \odot$ & -7.0261 \\
\hline $\mathrm{CaF}+$ & $5.024 \mathrm{e}-\odot \odot 8$ & ๑. $\odot \odot 2967$ & 0.9422 & -7.3248 \\
\hline KSO4 - & $3.361 \mathrm{e}-008$ & $\odot .004542$ & 0.9422 & -7.4993 \\
\hline $\mathrm{MgF}+$ & $2.862 e-008$ & 0.001239 & 0.9422 & -7.5692 \\
\hline $\operatorname{NaF}(\mathrm{aq})$ & $1.313 e-008$ & ๑. 0005512 & 1.0000 & -7.8817 \\
\hline
\end{tabular}




\begin{tabular}{|c|c|c|c|}
\hline Mineral saturation & \multicolumn{2}{|c|}{$\log Q / K$} & $\log Q / K$ \\
\hline \multicolumn{4}{|c|}{ 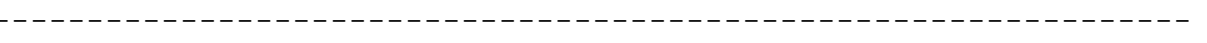 } \\
\hline Antigorite & $19.1995 \mathrm{~s} / \mathrm{sat}$ & Saponite-H & $3.8575 \mathrm{~s} / \mathrm{sat}$ \\
\hline Nontronite-Ca & $18.9376 \mathrm{~s} / \mathrm{sat}$ & Prehnite & $3.5301 \mathrm{~s} / \mathrm{sat}$ \\
\hline Nontronite-Mg & $18.8131 \mathrm{~s} / \mathrm{sat}$ & Sanidine_high & $3.4867 \mathrm{~s} / \mathrm{sat}$ \\
\hline Nontronite-Na & $18.6363 \mathrm{~s} / \mathrm{sat}$ & Tremolite & $3.1981 \mathrm{~s} / \mathrm{sat}$ \\
\hline Nontronite-K & $18.4935 \mathrm{~s} / \mathrm{sat}$ & Albite_low & $3.0993 \mathrm{~s} / \mathrm{sat}$ \\
\hline Nontronite-H & $17.6372 \mathrm{~s} / \mathrm{sat}$ & Albite & 3.0992s/sat \\
\hline Clinoptilolite-h & $16.7414 \mathrm{~s} / \mathrm{sat}$ & Smectite-low-Fe- & $3.0174 \mathrm{~s} / \mathrm{sat}$ \\
\hline Clinoptilolite-C & $16.7123 \mathrm{~s} / \mathrm{sat}$ & Natrolite & $2.8830 \mathrm{~s} / \mathrm{sat}$ \\
\hline Clinoptilolite-h & $14.2866 \mathrm{~s} / \mathrm{sat}$ & Clinozoisite & $2.6030 \mathrm{~s} / \mathrm{sat}$ \\
\hline Clinoptilolite-N & $14.2858 \mathrm{~s} / \mathrm{sat}$ & Zoisite & $2.5571 \mathrm{~s} / \mathrm{sat}$ \\
\hline Hematite & $13.5895 \mathrm{~s} / \mathrm{sat}$ & Diaspore & $2.3470 \mathrm{~s} / \mathrm{sat}$ \\
\hline Clinoptilolite-K & $13.0806 \mathrm{~s} / \mathrm{sat}$ & Witherite & $2.1489 \mathrm{~s} / \mathrm{sat}$ \\
\hline Clinoptilolite-h & $12.9708 \mathrm{~s} / \mathrm{sat}$ & Magnetite & $2.1250 \mathrm{~s} / \mathrm{sat}$ \\
\hline Stilbite & $12.5173 \mathrm{~s} / \mathrm{sat}$ & Boehmite & $1.9345 \mathrm{~s} / \mathrm{sat}$ \\
\hline Andradite & $11.3900 \mathrm{~s} / \mathrm{sat}$ & Gibbsite & $1.7750 \mathrm{~s} / \mathrm{sat}$ \\
\hline Epidote-ord & $10.3501 \mathrm{~s} / \mathrm{sat}$ & Albite_high & $1.7470 \mathrm{~s} / \mathrm{sat}$ \\
\hline Epidote & $10.3496 \mathrm{~s} / \mathrm{sat}$ & Clinochlore-7A & $1.7366 \mathrm{~s} / \mathrm{sat}$ \\
\hline Muscovite & $9.8777 \mathrm{~s} / \mathrm{sat}$ & Wairakite & $1.4713 \mathrm{~s} / \mathrm{sat}$ \\
\hline Mesolite & $8.6712 \mathrm{~s} / \mathrm{sat}$ & Dolomite & 1.0503s/sat \\
\hline Herschelite & $8.3903 \mathrm{~s} / \mathrm{sat}$ & Dolomite-ord & $1.0503 \mathrm{~s} / \mathrm{sat}$ \\
\hline Illite & $7.4919 \mathrm{~s} / \mathrm{sat}$ & Quartz & $\odot .7891 \mathrm{~s} / \mathrm{sat}$ \\
\hline Paragonite & $7.3478 \mathrm{~s} / \mathrm{sat}$ & Jadeite & $0.6863 \mathrm{~s} / \mathrm{sat}$ \\
\hline Scolecite & $7.0796 \mathrm{~s} / \mathrm{sat}$ & Corundum & $0.5950 \mathrm{~s} / \mathrm{sat}$ \\
\hline Beidellite-Ca & $6.9807 \mathrm{~s} / \mathrm{sat}$ & Tridymite & $\odot .5915 \mathrm{~s} / \mathrm{sat}$ \\
\hline Montmor-Ca & $6.9344 \mathrm{~s} / \mathrm{sat}$ & Calcite & $0.4097 \mathrm{~s} / \mathrm{sat}$ \\
\hline Montmor-Mg & $6.8813 \mathrm{~s} / \mathrm{sat}$ & Smectite-high-Fe & $0.3931 \mathrm{~s} / \mathrm{sat}$ \\
\hline Beidellite-Mg & $6.8561 \mathrm{~s} / \mathrm{sat}$ & Chrysotile & $0.2893 \mathrm{~s} / \mathrm{sat}$ \\
\hline Montmor-Na & $6.7010 \mathrm{~s} / \mathrm{sat}$ & Aragonite & $0.2651 \mathrm{~s} / \mathrm{sat}$ \\
\hline Beidellite-Na & $6.6793 \mathrm{~s} / \mathrm{sat}$ & Cristobalite(alp & $0.2271 \mathrm{~s} / \mathrm{sat}$ \\
\hline Montmor-K & $6.5622 \mathrm{~s} / \mathrm{sat}$ & Chalcedony & $0.2132 \mathrm{~s} / \mathrm{sat}$ \\
\hline Beidellite-K & $6.5366 \mathrm{~s} / \mathrm{sat}$ & Kyanite & $-\odot . \odot \odot \odot 6$ \\
\hline Amesite-14A & $6.4670 \mathrm{~s} / \mathrm{sat}$ & Coesite & -0.0341 \\
\hline Celadonite & $6.4208 \mathrm{~s} / \mathrm{sat}$ & Kalsilite & -0.0907 \\
\hline Goethite & $6.3202 \mathrm{~s} / \mathrm{sat}$ & Dawsonite & -0.1190 \\
\hline Laumontite & $6.0418 \mathrm{~s} / \mathrm{sat}$ & Ice & -0.1194 \\
\hline Kaolinite & $5.7920 \mathrm{~s} / \mathrm{sat}$ & Cristobalite(bet & -0.2309 \\
\hline Pyrophyllite & $5.6940 \mathrm{~s} / \mathrm{sat}$ & Analcime-K & -0.2541 \\
\hline Beidellite-H & $5.6804 \mathrm{~s} / \mathrm{sat}$ & Andalusite & -0.2841 \\
\hline Ferrite-Ca & $5.5915 \mathrm{~s} / \mathrm{sat}$ & Monohydrocalcite & -0.4110 \\
\hline Saponite-Ca & $5.1578 \mathrm{~s} / \mathrm{sat}$ & Dolomite-dis & -0.5315 \\
\hline Clinochlore-14A & $5.1516 \mathrm{~s} / \mathrm{sat}$ & $\mathrm{SiO} 2(\mathrm{am})$ & $-\odot .5390$ \\
\hline Ferrite-Mg & $5.0856 \mathrm{~s} / \mathrm{sat}$ & Anorthite & -0.6071 \\
\hline Saponite-Mg & $5.0339 \mathrm{~s} / \mathrm{sat}$ & Sillimanite & -0.6576 \\
\hline Saponite-Na & $4.8566 \mathrm{~s} / \mathrm{sat}$ & Analcime & -0.8093 \\
\hline Phlogopite & $4.8330 \mathrm{~s} / \mathrm{sat}$ & Magnesite & -1.0194 \\
\hline Maximum_Microcli & $4.7198 \mathrm{~s} / \mathrm{sat}$ & Barite & -1.1747 \\
\hline K-Feldspar & $4.7186 \mathrm{~s} / \mathrm{sat}$ & Diopside & -1.2652 \\
\hline Saponite-K & $4.7138 \mathrm{~s} / \mathrm{sat}$ & Nepheline & -1.5690 \\
\hline Mordenite & $3.9558 \mathrm{~s} / \mathrm{sat}$ & Enstatite & -1.9891 \\
\hline Lawsonite & $3.9339 \mathrm{~s} / \mathrm{sat}$ & Grossular & $-2.00 \odot 4$ \\
\hline Margarite & $3.9240 \mathrm{~s} / \mathrm{sat}$ & Fluorite & -2.0407 \\
\hline Talc & $3.8681 \mathrm{~s} / \mathrm{sat}$ & $\mathrm{Fe}(\mathrm{OH}) 3$ & -2.3676 \\
\hline & h & 3 listed) & \\
\hline
\end{tabular}




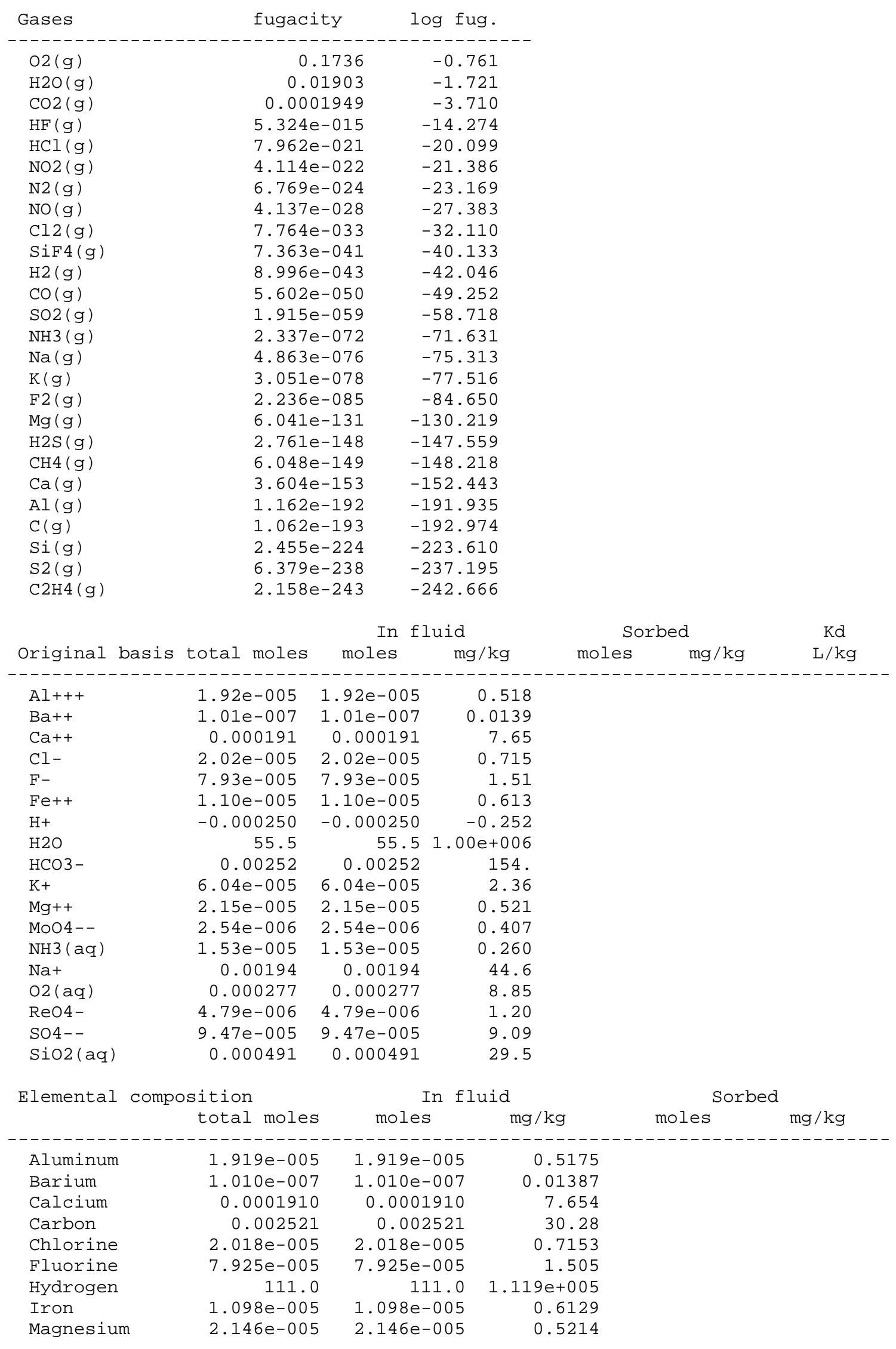




$\begin{array}{lrrr}\text { Molybdenum } & 2.544 \mathrm{e}-\odot \odot 6 & 2.544 \mathrm{e}-006 & 0.244 \odot \\ \text { Nitrogen } & 1.528 \mathrm{e}-005 & 1.528 \mathrm{e}-005 & 0.2140 \\ \text { Oxygen } & 55.52 & 55.52 & 8.880 \mathrm{e}+005 \\ \text { Potassium } & 6.041 \mathrm{e}-005 & 6.041 \mathrm{e}-005 & 2.361 \\ \text { Rhenium } & 4.788 \mathrm{e}-006 & 4.788 \mathrm{e}-006 & 0.8914 \\ \text { Silicon } & 0.0004906 & 0.0004906 & 13.77 \\ \text { Sodium } & 0.001939 & 0.001939 & 44.57 \\ \text { Sulfur } & 9.466 \mathrm{e}-005 & 9.466 \mathrm{e}-005 & 3.035\end{array}$




\section{Sample D1019MMB_ts}

$\begin{array}{ll}\text { Step \# } \odot & \\ \text { Temperature }=20 . \odot \mathrm{C} \\ \text { pH }=9.840 \\ \text { Eh = } 0.6468 \text { volts } \\ \text { Ionic strength } \\ \text { Charge imbalance }= \\ \text { Activity of water }= \\ \text { Solvent mass } & = \\ \text { Solution mass } & = \\ \text { Solution density } & = \\ \text { Chlorinity } & = \\ \text { Dissolved solids } & = \\ \text { Elect. conductivity } & = \\ \text { Hardness } & = \\ \text { carbonate } & = \\ \text { non-carbonate } & = \\ \text { Rock mass } & = \\ \text { Carbonate alkalinity } & = \\ \text { Water type } & =\end{array}$

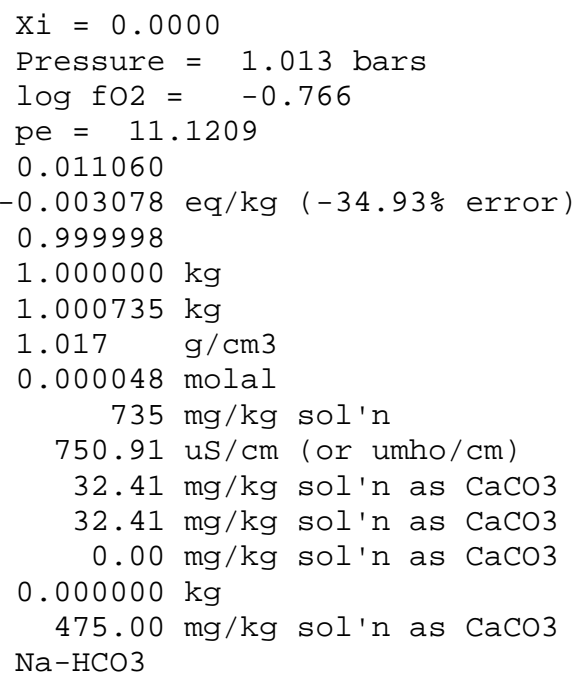

No minerals in system.

\begin{tabular}{|c|c|c|c|c|}
\hline Aqueous species & molality & $\mathrm{mg} / \mathrm{kg}$ sol'n & act. coef. & $\log$ act \\
\hline - - - - - - - - - - - & ----- & $\ldots-\ldots$ & - & \\
\hline $\mathrm{Na}+$ & $\odot .006952$ & 159.7 & ๑. 8988 & -2.2042 \\
\hline $\mathrm{HCO} 3-$ & 0.005121 & 312.3 & $\odot .8988$ & -2.3369 \\
\hline CO3 - - & $\odot .0 \odot 2009$ & 120.5 & 0.6548 & -2.8809 \\
\hline $\operatorname{Si02}(\mathrm{aq})$ & 0.0003566 & 21.41 & 1.0000 & -3.4478 \\
\hline BO2- & 0.0002876 & 12.30 & 0.8988 & -3.5876 \\
\hline HSi03- & $\odot . \odot \odot \odot 269 \odot$ & 20.72 & $\odot .8988$ & -3.6167 \\
\hline $02(a q)$ & $\odot .0002400$ & 7.675 & 1.0000 & -3.6197 \\
\hline $\mathrm{F}-$ & 0.0002117 & 4.019 & 0.8973 & -3.7214 \\
\hline CaC03(aq) & ๑ . 0001823 & 18.23 & 1.0000 & -3.7392 \\
\hline $\mathrm{Ca}++$ & ๑. . 0001077 & 4.312 & 0.6668 & -4.1439 \\
\hline SO4 - - & $8.884 e-005$ & 8.528 & 0.6506 & -4.2381 \\
\hline NaHSiO3 $(\mathrm{aq})$ & $7.544 \mathrm{e}-\odot \odot 5$ & 7.545 & $1.0 \odot \odot \odot$ & -4.1224 \\
\hline $\mathrm{B}(\mathrm{OH}) 3(\mathrm{aq})$ & $7.481 e-005$ & 4.622 & 1.0000 & -4.1260 \\
\hline $\mathrm{K}+\mathrm{C}$ & $6.845 e-005$ & 2.674 & $\odot .8958$ & -4.2124 \\
\hline N03 - & $5.350 \mathrm{e}-\odot \odot 5$ & 3.315 & $\odot .8958$ & -4.3194 \\
\hline $\mathrm{OH}-$ & $5.165 e-005$ & 0.8778 & $\odot .8973$ & -4.3340 \\
\hline $\mathrm{Cl}-$ & $4.771 e-005$ & 1.690 & 0.8958 & -4.3692 \\
\hline $\mathrm{NaHCO} 3(\mathrm{aq})$ & $4.537 e-005$ & 3.808 & 1.0000 & -4.3433 \\
\hline Al02 - & $4.044 \mathrm{e}-005$ & 2.383 & 0.8988 & -4.4395 \\
\hline $\mathrm{NaCO} 3-$ & $3.453 e-005$ & 2.864 & $\odot .8988$ & -4.5081 \\
\hline $\mathrm{Fe}(\mathrm{OH}) 3(\mathrm{aq})$ & $2.203 e-005$ & 2.353 & 1.0000 & -4.6569 \\
\hline $\mathrm{Mg}++$ & $1.569 e-005$ & 0.3811 & 0.6816 & -4.9708 \\
\hline $\operatorname{MgC03}(\mathrm{aq})$ & $1.283 e-005$ & 1.081 & 1.0000 & -4.8917 \\
\hline Re04 - & $1.269 \mathrm{e}-\odot \odot 5$ & 3.172 & 0.8988 & -4.9430 \\
\hline HPO4 - - & $8.226 e-0 \odot 6$ & 0.7889 & 0.6506 & -5.2715 \\
\hline Mo04 - - & $7.028 e-0 \odot 6$ & 1.123 & 0.6548 & -5.3370 \\
\hline CaHCO3+ & $4.174 \mathrm{e}-006$ & 0.4217 & ๑. 8988 & -5.4257 \\
\hline $\mathrm{Zn++}$ & $3.273 e-006$ & 0.2139 & 0.6668 & -5.6610 \\
\hline $\mathrm{co2}(\mathrm{aq})$ & $1.623 e-006$ & ๑. 07137 & $1.000 \odot$ & -5.7897 \\
\hline Se04-- & $1.111 \mathrm{e}-006$ & $\odot .1587$ & 0.6506 & -6.1411 \\
\hline $\mathrm{MgHCO} 3+$ & $6.073 e-0 \odot 7$ & ๑. 05178 & 0.8988 & -6.2629 \\
\hline CaSo4(aq) & $5.245 e-0 \odot 7$ & ๑. 07135 & 1.0000 & -6.2803 \\
\hline $\operatorname{MgSO} 4(\mathrm{aq})$ & $1.407 e-007$ & ๑. 01693 & 1.0000 & -6.8516 \\
\hline $\mathrm{Ba}++$ & $1.337 \mathrm{e}-0 \odot 7$ & 0.01835 & $\odot .6589$ & -7.0551 \\
\hline $\operatorname{NaF}(\mathrm{aq})$ & $1.150 \mathrm{e}-007$ & ๑. .0०4825 & 1.0000 & -6.9393 \\
\hline $\mathrm{CaF}+$ & $7.183 e-008$ & $\odot .004240$ & 0.8988 & -7.1900 \\
\hline $\mathrm{MgF}+$ & $5.166 e-0 \odot 8$ & $\odot .002236$ & 0.8988 & -7.3331 \\
\hline
\end{tabular}




\begin{tabular}{|c|c|c|c|c|}
\hline $\mathrm{NaOH}(\mathrm{aq})$ & $4.779 e-\odot \odot 8$ & ๑. .001910 & $1.00 \odot \odot$ & -7.3207 \\
\hline $\operatorname{BaC03}(\mathrm{aq})$ & $4.622 e-008$ & ๑.009113 & 1.0000 & -7.3352 \\
\hline $\operatorname{NaCl}(\mathrm{aq})$ & $4.354 e-008$ & 0.002543 & 1.0000 & -7.3611 \\
\hline P04-- - & $4.114 \mathrm{e}-\odot \odot 8$ & 0.003904 & 0.3797 & -7.8063 \\
\hline $\operatorname{NaAl02}(\mathrm{aq})$ & $3.914 \mathrm{e}-008$ & 0.003206 & 1.0000 & -7.4073 \\
\hline KSO4 - & $2.997 e-\odot \odot 8$ & 0.004047 & ๑. 8988 & -7.5697 \\
\hline ZnS04(aq) & $2.335 e-008$ & 0.003767 & 1.0000 & -7.6317 \\
\hline HAlO2 ( $\mathrm{aq})$ & $1.948 \mathrm{e}-0 \odot 8$ & ๑ . 001168 & 1.0000 & -7.7105 \\
\hline $\mathrm{H} 2 \mathrm{PO} 4-$ & 1. $45 \odot \mathrm{e}-\odot \odot 8$ & ๑. .001406 & ๑. 8988 & -7.8848 \\
\hline
\end{tabular}

$\begin{aligned} \text { Mineral saturation } & \text { states } \\ & \log Q / K\end{aligned}$

$\log \mathrm{Q} / \mathrm{K}$

\begin{tabular}{|c|c|c|c|}
\hline Antigorite & $102.4991 \mathrm{~s} / \mathrm{sat}$ & Clinozoisite & $3.6515 \mathrm{~s} / \mathrm{sa}$ \\
\hline Nontronite-Ca & $19.2128 \mathrm{~s} / \mathrm{sat}$ & Albite_low & $3.6257 \mathrm{~s} / \mathrm{sa}$ \\
\hline Nontronite-Na & $19.1333 \mathrm{~s} / \mathrm{sat}$ & Albite & $3.6256 \mathrm{~s} / \mathrm{sc}$ \\
\hline Nontronite-Mg & 19.1051s/sat & Zoisite & $3.6056 \mathrm{~s} / \mathrm{s}$ \\
\hline Nontronite-K & $18.8245 \mathrm{~s} / \mathrm{sat}$ & Sanidine_high & $3.5099 \mathrm{~s} / \mathrm{s}$ \\
\hline Nontronite-H & $17.6306 \mathrm{~s} / \mathrm{sat}$ & Witherite & $3.4988 \mathrm{~s} / \mathrm{s}$ \\
\hline Andradite & $16.8055 \mathrm{~s} / \mathrm{sat}$ & Dolomite & $3.2059 \mathrm{~s} /$ \\
\hline Ferrite-Zn & $16.2927 \mathrm{~s} / \mathrm{sat}$ & Dolomite-ord & $3.2059 \mathrm{~s} / \mathrm{s}$ \\
\hline Clinoptilolite-h & $15.8192 \mathrm{~s} / \mathrm{sat}$ & Magnetite & $3.0341 \mathrm{~s} / \mathrm{s}$ \\
\hline Clinoptilolite-C & $15.7902 \mathrm{~s} / \mathrm{sat}$ & Margarite & $2.6847 \mathrm{~s} / \mathrm{s}$ \\
\hline Clinoptilolite-h & $15.6940 \mathrm{~s} / \mathrm{sat}$ & Zincite & $2.5405 \mathrm{~s} / \mathrm{s}$ \\
\hline Clinoptilolite-N & $15.6932 \mathrm{~s} / \mathrm{sat}$ & Albite_high & $2.2734 \mathrm{~s} / \mathrm{s}$ \\
\hline Tremolite & $14.8236 \mathrm{~s} / \mathrm{sat}$ & $\mathrm{Zn}(\mathrm{OH}) \overline{2}$ (gamma $)$ & $2.1358 \mathrm{~s} /$ \\
\hline Hematite & $14.1946 \mathrm{~s} / \mathrm{sat}$ & $\mathrm{Zn}(\mathrm{OH}) 2$ (epsilon) & $2.1105 \mathrm{~s} /$ \\
\hline Fluorapatite & $14.0303 \mathrm{~s} / \mathrm{sat}$ & Diopside & $2.0430 \mathrm{~s} /$ \\
\hline Clinoptilolite-K & $12.7433 \mathrm{~s} / \mathrm{sat}$ & Smectite-high-Fe & $1.8634 \mathrm{~s} / \mathrm{s}$ \\
\hline Clinoptilolite-h & $12.6335 \mathrm{~s} / \mathrm{sat}$ & $\mathrm{Zn}(\mathrm{OH}) 2($ beta $)$ & $1.8346 \mathrm{~s} / \mathrm{s}$ \\
\hline Clinochlore-14A & $12.5168 \mathrm{~s} / \mathrm{sat}$ & Diaspore & $1.6626 \mathrm{~s} /$ \\
\hline Epidote-ord & $12.3855 \mathrm{~s} / \mathrm{sat}$ & Dolomite-dis & $1.6241 \mathrm{~s} /$ \\
\hline Epidote & $12.3851 \mathrm{~s} / \mathrm{sat}$ & Grossular & $1.4411 \mathrm{~s} /$ \\
\hline Stilbite & $12.2630 \mathrm{~s} / \mathrm{sat}$ & Calcite & $1.4368 \mathrm{~s} /$ \\
\hline Amesite-14A & $10.7586 \mathrm{~s} / \mathrm{sat}$ & Wairakite & $1.3906 \mathrm{~s} /$ \\
\hline Zn2Si04 & $10.3751 \mathrm{~s} / \mathrm{sat}$ & Jadeite & $1.3178 \mathrm{~s} /$ \\
\hline Phlogopite & $10.2858 \mathrm{~s} / \mathrm{sat}$ & Aragonite & $1.2923 \mathrm{~s} /$ \\
\hline Saponite-Ca & $10.2577 \mathrm{~s} / \mathrm{sat}$ & Smithsonite & $1.2912 \mathrm{~s} /$ \\
\hline Saponite-Na & $10.1781 \mathrm{~s} / \mathrm{sat}$ & Boehmite & $1.2501 \mathrm{~s}$ \\
\hline Saponite-Mg & $10.1505 \mathrm{~s} / \mathrm{sat}$ & Gibbsite & $1.0906 \mathrm{~s} /$ \\
\hline Saponite-K & $9.8693 \mathrm{~s} / \mathrm{sat}$ & Quartz & $0.6840 \mathrm{~s} /$ \\
\hline Herschelite & $9.4070 \mathrm{~s} / \mathrm{sat}$ & Monohydrocalcite & $0.6161 \mathrm{~s} / \mathrm{s}$ \\
\hline Mesolite & $9.1470 \mathrm{~s} / \mathrm{sat}$ & Whitlockite & $0.6030 \mathrm{~s} / \mathrm{s}$ \\
\hline Clinochlore-7A & $9.1018 \mathrm{~s} / \mathrm{sat}$ & Tridymite & $0.4864 \mathrm{~s} /$ \\
\hline Talc & $8.8772 \mathrm{~s} / \mathrm{sat}$ & Huntite & $\odot .1560 \mathrm{~s} /$ \\
\hline Saponite-H & $8.6755 \mathrm{~s} / \mathrm{sat}$ & Kalsilite & $0.1428 \mathrm{~s} /$ \\
\hline Muscovite & $8.5321 \mathrm{~s} / \mathrm{sat}$ & Cristobalite(alp & $0.1220 \mathrm{~s} /$ \\
\hline Celadonite & $8.1487 \mathrm{~s} / \mathrm{sat}$ & Magnesite & $0.1090 \mathrm{~s} /$ \\
\hline Ferrite-Ca & 7.9051s/sat & Chalcedony & $0.1081 \mathrm{~s} /$ \\
\hline Ferrite-Mg & $7.5006 \mathrm{~s} / \mathrm{sat}$ & Dawsonite & $0.0414 \mathrm{~s} /$ \\
\hline Anthophyllite & 7.3778s/sat & Ice & -0.1194 \\
\hline Scolecite & $7.1040 \mathrm{~s} / \mathrm{sat}$ & Coesite & -0.1393 \\
\hline Goethite & $6.6228 \mathrm{~s} / \mathrm{sat}$ & Pargasite & -0.1427 \\
\hline Illite & $6.6160 \mathrm{~s} / \mathrm{sat}$ & Analcime-K & -0.1435 \\
\hline Paragonite & $6.5054 \mathrm{~s} / \mathrm{sat}$ & Analcime & -0.2156 \\
\hline Montmor-Ca & $6.2500 \mathrm{~s} / \mathrm{sat}$ & Enstatite & -0.2843 \\
\hline Montmor-Na & $6.2384 \mathrm{~s} / \mathrm{sat}$ & Cristobalite (bet & -0.3361 \\
\hline Montmor-Mg & $6.2137 \mathrm{~s} / \mathrm{sat}$ & Anorthite & -0.4776 \\
\hline Laumontite & $5.9611 \mathrm{~s} / \mathrm{sat}$ & Si02(am) & -0.6441 \\
\hline Montmor-K & $5.9335 \mathrm{~s} / \mathrm{sat}$ & Corundum & -0.7738 \\
\hline Chrysotile & $5.5087 \mathrm{~s} / \mathrm{sat}$ & Nepheline & -0.8324 \\
\hline Hydroxylapatite & $5.3567 \mathrm{~s} / \mathrm{sat}$ & Barite & -1.2267 \\
\hline
\end{tabular}




$\begin{array}{lllr}\text { Beidellite-Ca } & 5.2821 \mathrm{~s} / \mathrm{sat} & \text { Kyanite } & -1.4746 \\ \text { Prehnite } & 5.2630 \mathrm{~s} / \mathrm{sat} & \text { Fluorite } & -1.4997 \\ \text { Beidellite-Na } & 5.2025 \mathrm{~s} / \mathrm{sat} & \text { Okenite } & -1.7416 \\ \text { Beidellite-Mg } & 5.1743 \mathrm{~s} / \mathrm{sat} & \text { Andalusite } & -1.7581 \\ \text { Beidellite-K } & 4.8937 \mathrm{~s} / \mathrm{sat} & \text { Wollastonite } & -1.8735 \\ \text { Maximum_Microcli } & 4.7430 \mathrm{~s} / \mathrm{sat} & \text { Brucite } & -1.9277 \\ \text { K-Feldspar } & 4.7418 \mathrm{~s} / \mathrm{sat} & \text { Fe(OH)3 } & -2.0651 \\ \text { Natrolite } & 4.2512 \mathrm{~s} / \mathrm{sat} & \text { Pseudowollastoni } & -2.1216 \\ \text { Kaolinite } & 4.2129 \mathrm{~s} / \mathrm{sat} & \text { Sillimanite } & -2.1315 \\ \text { Lawsonite } & 4.0634 \mathrm{~s} / \mathrm{sat} & \text { Gyrolite } & -2.3409 \\ \text { Smectite-low-Fe- } & 4.0008 \mathrm{~s} / \mathrm{sat} & \text { Forsterite } & -2.4839 \\ \text { Pyrophyllite } & 3.9046 \mathrm{~s} / \mathrm{sat} & \text { Cordierite_hydr } & -2.6993 \\ \text { Mordenite } & 3.8260 \mathrm{~s} / \mathrm{sat} & \text { Artinite } & -2.8073 \\ \text { Beidellite-H } & 3.6999 \mathrm{~s} / \mathrm{sat} & \text { Nesquehonite } & -2.9096 \\ \quad \text { (only minerals with } \mathrm{log} \text { Q/K > } & -3 \text { listed) } & \end{array}$

\begin{tabular}{|c|c|c|}
\hline Gases & fugacity & $\log$ fug. \\
\hline $02(a)$ & 0.1712 & -0.766 \\
\hline $\mathrm{H} 2 \mathrm{O}(\mathrm{g})$ & ๑. . 01903 & -1.721 \\
\hline $\operatorname{co2}(\mathrm{g})$ & $4.059 \mathrm{e}-\odot \odot 5$ & -4.392 \\
\hline$H F(g)$ & $1.388 e-015$ & -14.858 \\
\hline $\mathrm{HCl}(\mathrm{g})$ & $1.834 \mathrm{e}-021$ & -20.737 \\
\hline N02 (g) & $1.407 \mathrm{e}-022$ & -21.852 \\
\hline $\mathrm{N} 2(\mathrm{~g})$ & $8.135 e-025$ & -24.090 \\
\hline NO $(g)$ & $1.424 \mathrm{e}-028$ & -27.846 \\
\hline $\mathrm{Cl} 2(\mathrm{~g})$ & $4.091 \mathrm{e}-034$ & -33.388 \\
\hline $\mathrm{H} 2(\mathrm{~g})$ & $9.056 e-043$ & -42.043 \\
\hline $\mathrm{BF} 3(\mathrm{~g})$ & $8.031 e-043$ & -42.095 \\
\hline SiF4(g) & $2.670 \mathrm{e}-\odot 43$ & -42.573 \\
\hline $\mathrm{CO}(\mathrm{g})$ & $1.174 \mathrm{e}-050$ & -49.930 \\
\hline $\mathrm{S} 02(\mathrm{~g})$ & $1.591 \mathrm{e}-061$ & -60.798 \\
\hline $\mathrm{Zn}(\mathrm{g})$ & $5.327 e-072$ & -71.274 \\
\hline $\mathrm{NH3}(\mathrm{g})$ & $8.184 \mathrm{e}-073$ & -72.087 \\
\hline $\mathrm{Na}(\mathrm{g})$ & $1.639 e-074$ & -73.785 \\
\hline$K(g)$ & $3.227 e-077$ & -76.491 \\
\hline $\mathrm{F} 2(\mathrm{~g})$ & $1.510 \mathrm{e}-086$ & -85.821 \\
\hline $\operatorname{Mg}(g)$ & $3.925 e-129$ & -128.406 \\
\hline $\mathrm{CH} 4(\mathrm{~g})$ & $1.294 \mathrm{e}-149$ & -148.888 \\
\hline $\mathrm{H} 2 \mathrm{~S}(\mathrm{~g})$ & $2.341 \mathrm{e}-150$ & -149.631 \\
\hline $\mathrm{Ca}(\mathrm{g})$ & $1.855 e-151$ & -150.732 \\
\hline$A l(g)$ & $2.428 e-193$ & -192.615 \\
\hline$C(g)$ & $2.242 \mathrm{e}-194$ & -193.649 \\
\hline$B(g)$ & $1.396 \mathrm{e}-206$ & -205.855 \\
\hline Si(g) & $1.954 \mathrm{e}-224$ & -223.709 \\
\hline $\mathrm{S} 2(\mathrm{~g})$ & $4.524 \mathrm{e}-242$ & -241.344 \\
\hline $\mathrm{C} 2 \mathrm{H} 4(\mathrm{~g})$ & $9.743 e-245$ & -244.011 \\
\hline
\end{tabular}

\begin{tabular}{|c|c|c|c|c|c|}
\hline \multirow[b]{2}{*}{ Original basis } & \multirow[b]{2}{*}{ total moles } & \multicolumn{2}{|c|}{ In fluid } & Sorbed & \multirow{2}{*}{$\begin{array}{c}\mathrm{Kd} \\
\mathrm{L} / \mathrm{kg}\end{array}$} \\
\hline & & moles & $\mathrm{mg} / \mathrm{kg}$ & moles $\quad \mathrm{mg} / \mathrm{kg}$ & \\
\hline - & $2-5-2$ & $---1----1$ & $-\ldots$ & & \\
\hline $\mathrm{Al}+++$ & $4.05 e-005$ & $4.05 e-005$ & 1.09 & & \\
\hline $\mathrm{B}(\mathrm{OH}) 3(\mathrm{aq})$ & 0.000362 & 0.000362 & 22.4 & & \\
\hline $\mathrm{Ba}++$ & $1.80 \mathrm{e}-0 \odot 7$ & $1.80 \mathrm{e}-\odot \odot 7$ & 0.0247 & & \\
\hline $\mathrm{Ca}++$ & ๑. 000295 & 0.000295 & 11.8 & & \\
\hline $\mathrm{Cl}-$ & $4.78 e-0 \odot 5$ & $4.78 \mathrm{e}-\odot \odot 5$ & 1.69 & & \\
\hline $\mathrm{F}-$ & $\odot .0 \odot \odot 212$ & $\odot .000212$ & 4.02 & & \\
\hline $\mathrm{Fe}++$ & $2.20 \mathrm{e}-\odot \odot 5$ & $2.20 \mathrm{e}-0 \odot 5$ & 1.23 & & \\
\hline $\mathrm{H}+$ & -0.00318 & -0.00318 & -3.20 & & \\
\hline $\mathrm{H} 2 \mathrm{O}$ & 55.5 & 55.5 & $9.99 e+005$ & & \\
\hline HCO3 - & 0.00741 & 0.00741 & 452 & & \\
\hline HPO4 - - & $8.28 e-006$ & $8.28 e-0 \odot 6$ & 0.794 & & \\
\hline $\mathrm{K}+$ & $6.85 e-005$ & $6.85 e-005$ & 2.68 & & \\
\hline
\end{tabular}




\begin{tabular}{|c|c|c|c|c|}
\hline $\mathrm{Mg}++$ & $2.93 e-0 \odot 5$ & $2.93 e-\odot \odot 5$ & \multicolumn{2}{|l|}{0.712} \\
\hline Mo04-- & $7.03 e-0 \odot 6$ & $7.03 e-\odot \odot 6$ & \multicolumn{2}{|l|}{1.12} \\
\hline NH3 $(a q)$ & $5.35 e-005$ & $5.35 e-005$ & \multicolumn{2}{|l|}{0.910} \\
\hline $\mathrm{Na}+$ & 0.00711 & 0.00711 & \multicolumn{2}{|l|}{$\begin{array}{c}0.910 \\
163 .\end{array}$} \\
\hline $02(\mathrm{aq})$ & $\odot .00 \odot 353$ & $\odot .000353$ & \multicolumn{2}{|l|}{11.3} \\
\hline Re04- & $1.27 \mathrm{e}-005$ & & \multicolumn{2}{|l|}{3.17} \\
\hline SO4 - - & $8.96 e-005$ & $\begin{array}{l}1.27 \mathrm{e}-005 \\
8.96 \mathrm{e}-005\end{array}$ & \multicolumn{2}{|l|}{8.60} \\
\hline Se03-- & $1.11 \mathrm{e}-006$ & $\begin{array}{l}8.96 \mathrm{e}-005 \\
1.11 \mathrm{e}-006\end{array}$ & \multicolumn{2}{|l|}{0.141} \\
\hline $\operatorname{Si02}(\mathrm{aq})$ & 0.000701 & $\odot .000701$ & \multicolumn{2}{|l|}{42.1} \\
\hline $\mathrm{Zn++}$ & $3.30 \mathrm{e}-\odot \odot 6$ & $3.30 \mathrm{e}-006$ & \multicolumn{2}{|l|}{0.215} \\
\hline \multicolumn{2}{|c|}{ Elemental composition } & \multicolumn{2}{|c|}{ In fluid } & Sorbed \\
\hline & total moles & moles & $\mathrm{mg} / \mathrm{kg}$ & moles $\quad \mathrm{mg} / \mathrm{kg}$ \\
\hline Aluminum & $4.050 \mathrm{e}-005$ & $4.050 \mathrm{e}-005$ & 1.092 & \\
\hline Barium & $1.799 \mathrm{e}-\odot \odot 7$ & $1.799 \mathrm{e}-007$ & $\odot .02469$ & \\
\hline Boron & $\odot .0003624$ & $\odot .0003624$ & 3.915 & \\
\hline Calcium & $\odot .0 \odot \odot 2947$ & $\odot .00 \odot 2947$ & 11.80 & \\
\hline Carbon & $\odot .0 \odot 7412$ & 0.007412 & 88.96 & \\
\hline Chlorine & $4.776 e-\odot \odot 5$ & $4.776 e-005$ & 1.692 & \\
\hline Fluorine & $\odot .0 \odot \odot 2119$ & $\odot .0 \odot \odot 2119$ & 4.023 & \\
\hline Hydrogen & 111.0 & 111.0 & $1.118 \mathrm{e}+\odot \odot 5$ & \\
\hline Iron & $2.203 e-005$ & $2.203 e-005$ & 1.230 & \\
\hline Magnesium & $2.932 \mathrm{e}-\odot \odot 5$ & $2.932 \mathrm{e}-\odot \odot 5$ & 0.7122 & \\
\hline Molybdenum & $7 . \odot 28 e-\odot \odot 6$ & $7 . \odot 28 e-\odot \odot 6$ & 0.6738 & \\
\hline Nitrogen & $5.350 \mathrm{e}-005$ & $5.350 \mathrm{e}-005$ & 0.7488 & \\
\hline oxygen & 55.53 & 55.53 & $8.879 \mathrm{e}+\odot \odot 5$ & \\
\hline Phosphorus & $8.282 \mathrm{e}-006$ & $8.282 \mathrm{e}-006$ & 0.2563 & \\
\hline Potassium & $6.848 e-005$ & $6.848 e-005$ & 2.676 & \\
\hline Rhenium & 1. $269 \mathrm{e}-\odot \odot 5$ & $1.269 \mathrm{e}-005$ & 2.361 & \\
\hline Selenium & $1.111 \mathrm{e}-0 \odot 6$ & $1.111 \mathrm{e}-006$ & $\odot .08764$ & \\
\hline Silicon & $\odot .0 \odot \odot 7 \odot 1 \odot$ & $\odot .0 \odot \odot 7010$ & 19.67 & \\
\hline Sodium & ๑. 007108 & ๑. 007108 & 163.3 & \\
\hline Sulfur & ৪. 956e-๑๑5 & $8.956 \mathrm{e}-005$ & 2.870 & \\
\hline Zinc & $3.297 e-\odot \odot 6$ & $3.297 e-006$ & $\odot .2154$ & \\
\hline
\end{tabular}




\section{Sample D1019MMB_bs}

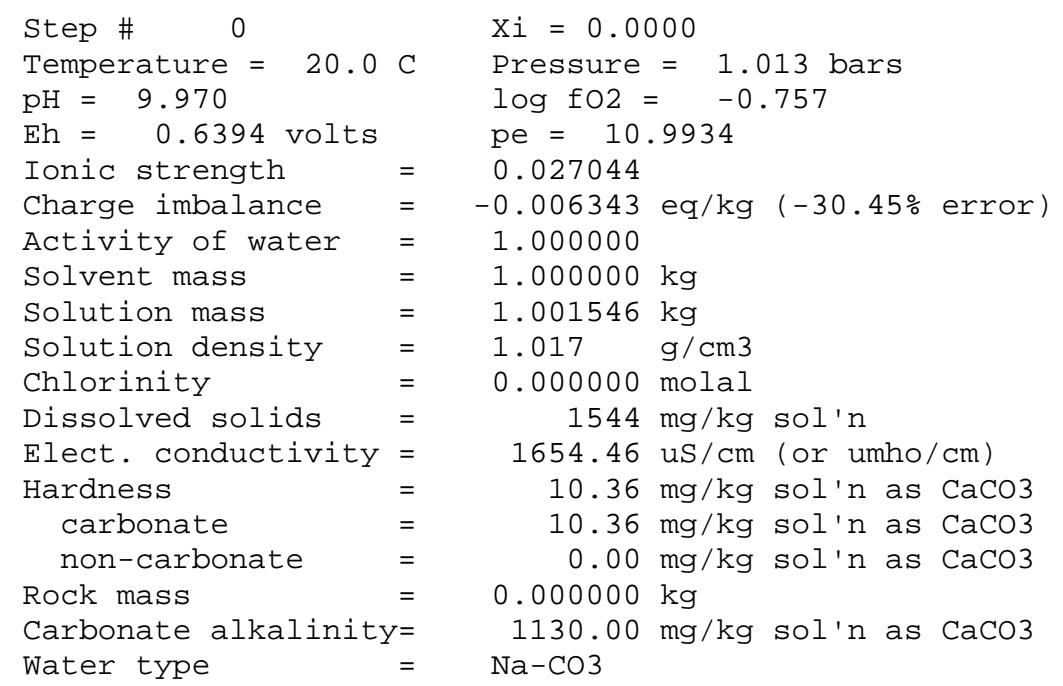

No minerals in system.

\begin{tabular}{|c|c|c|c|c|}
\hline Aqueous species & molality & $\mathrm{mg} / \mathrm{kg}$ sol'n & act. coef. & $\log$ act \\
\hline - - - - - - - - - - - & 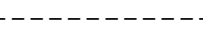 & $\ldots$ & - & \\
\hline $\mathrm{Na}+$ & 0.01749 & 401.5 & $\odot .8563$ & -1.8245 \\
\hline $\mathrm{HCO} 3-$ & $\odot .009877$ & 601.8 & $\odot .8563$ & -2.0727 \\
\hline CO3-- & 0.006029 & 361.2 & 0.5409 & -2.4867 \\
\hline B02- & 0.0009465 & 40.46 & 0.8563 & -3.0912 \\
\hline $\mathrm{F}-$ & 0.0003737 & 7.090 & 0.8532 & -3.4964 \\
\hline $02(\mathrm{aq})$ & $\odot .0002455$ & 7.844 & 1.0000 & -3.6099 \\
\hline $\mathrm{NaCO} 3-$ & $\odot .0002153$ & 17.85 & $\odot .8563$ & -3.7342 \\
\hline $\operatorname{NaHCO} 3(\mathrm{aq})$ & ๑. . 0001998 & 16.76 & 1.0000 & -3.6993 \\
\hline HSiO3- & ๑ . 0001959 & 15.08 & 0.8563 & -3.7753 \\
\hline Si02(aq) & ๑. . 0001835 & 11.01 & 1.0000 & -3.7364 \\
\hline $\mathrm{B}(\mathrm{OH}) 3(\mathrm{aq})$ & ๑. . 0001739 & 10.74 & 1.0000 & -3.7597 \\
\hline $\mathrm{K}+$ & $\odot . \odot \odot \odot 1387$ & 5.416 & $\odot .8500$ & -3.9284 \\
\hline NaHSiO3(aq) & ๑. . 0001255 & 12.54 & 1.0000 & -3.9013 \\
\hline SO4-- & $9.762 e-0 \odot 5$ & 9.363 & 0.5336 & -4.2833 \\
\hline $\mathrm{OH}-$ & $7.328 e-0 \odot 5$ & 1.244 & 0.8532 & -4.2040 \\
\hline Cac03(aq) & $6.398 e-005$ & 6.394 & 1.0000 & -4.1940 \\
\hline N03 - & 3. $891 e-005$ & 2.409 & 0.8500 & -4.4805 \\
\hline $\mathrm{Ca}++$ & $1.810 \mathrm{e}-005$ & 0.7241 & 0.5617 & -4.9929 \\
\hline Re04 - & $1.386 e-005$ & 3.462 & 0.8563 & -4.9257 \\
\hline HPO4 - - & $1.263 e-005$ & 1.210 & $\odot .5336$ & -5.1714 \\
\hline $\operatorname{MgC03}(\mathrm{aq})$ & $1.258 \mathrm{e}-005$ & 1.059 & 1.0000 & -4.9003 \\
\hline Mo04 - - & $9.865 e-006$ & 1.575 & 0.5409 & -5.2728 \\
\hline $\mathrm{Mg}++$ & $7.210 \mathrm{e}-006$ & 0.1750 & ๑. 5868 & -5.3736 \\
\hline Al02 - & $6.590 \mathrm{e}-006$ & 0.3881 & 0.8563 & -5.2485 \\
\hline $\mathrm{Zn++}$ & $2.547 \mathrm{e}-0 \odot 6$ & 0.1663 & $\odot .5617$ & -5.8445 \\
\hline $\operatorname{co2}(\mathrm{aq})$ & $2.211 \mathrm{e}-006$ & ๑. 09714 & 1.0000 & -5.6555 \\
\hline Se04-- & $1.409 \mathrm{e}-006$ & ๑. 2012 & 0.5336 & -6.1238 \\
\hline CaHCO3+ & $1.140 \mathrm{e}-006$ & 0.1151 & $\odot .8563$ & -6.0105 \\
\hline $\mathrm{MgHCO} 3+$ & $4.633 e-0 \odot 7$ & ๑. 03947 & $\odot .8563$ & -6.4015 \\
\hline $\operatorname{NaF}(\mathrm{aq})$ & $4.628 e-007$ & ๑. 01940 & 1.0000 & -6.3346 \\
\hline $\mathrm{NaOH}(\mathrm{aq})$ & $1.545 \mathrm{e}-007$ & $\odot .006172$ & 1.0000 & -6.8110 \\
\hline PO4 - - - & $1.094 \mathrm{e}-007$ & ๑. 01037 & 0.2426 & -7.5762 \\
\hline CaSo4(aq) & $6.692 e-008$ & ๑. 009096 & 1.0000 & -7.1744 \\
\hline $\mathrm{Ba}++$ & $6.322 \mathrm{e}-\odot \odot 8$ & ๑. 008668 & 0.5481 & -7.4603 \\
\hline KSO4 - & $5.451 e-0 \odot 8$ & $\odot .007357$ & 0.8563 & -7.3309 \\
\hline $\operatorname{MgS0} 4(\mathrm{aq})$ & $5.016 e-008$ & ๑. . 006029 & 1.0000 & -7.2996 \\
\hline $\operatorname{BaC0} 3(\mathrm{aq})$ & $4.506 e-008$ & $\odot .0 \odot 8878$ & 1.0000 & -7.3462 \\
\hline
\end{tabular}




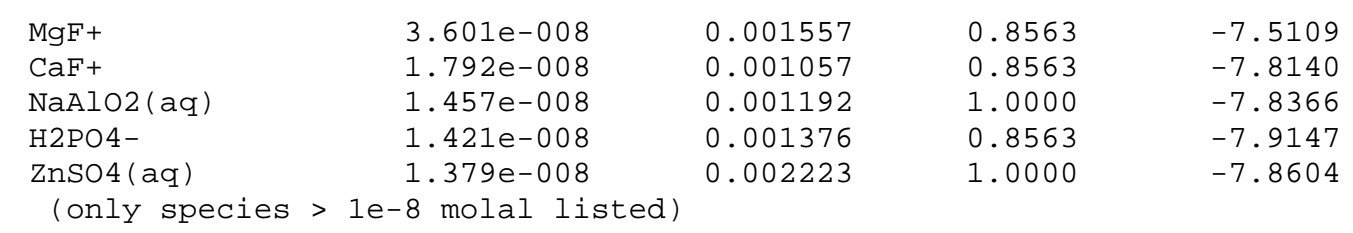

Mineral saturation states

$\log \mathrm{Q} / \mathrm{K} \quad \log \mathrm{Q} / \mathrm{K}$

\begin{tabular}{|c|c|c|c|}
\hline Antigorite & $85.8330 \mathrm{~s} / \mathrm{sat}$ & Kaolinite & $1.7577 \mathrm{~s} / \mathrm{sat}$ \\
\hline Fluorapatite & $10.7008 \mathrm{~s} / \mathrm{sat}$ & Smithsonite & $1.5019 \mathrm{~s} / \mathrm{sat}$ \\
\hline Tremolite & $10.6229 \mathrm{~s} / \mathrm{sat}$ & Mordenite & $1.4966 \mathrm{~s} / \mathrm{sat}$ \\
\hline Zn2SiO4 & $10.2395 \mathrm{~s} / \mathrm{sat}$ & Prehnite & $1.3413 \mathrm{~s} / \mathrm{sat}$ \\
\hline Clinochlore-14A & 9.0591s/sat & Dolomite-dis & $1.1607 \mathrm{~s} / \mathrm{sat}$ \\
\hline Saponite-Na & $8.5490 \mathrm{~s} / \mathrm{sat}$ & Lawsonite & $1.0193 \mathrm{~s} / \mathrm{sat}$ \\
\hline Phlogopite & $8.4667 \mathrm{~s} / \mathrm{sat}$ & Calcite & $\odot .9821 \mathrm{~s} / \mathrm{sat}$ \\
\hline Saponite-Ca & $8.3631 \mathrm{~s} / \mathrm{sat}$ & Albite_high & $0.9783 \mathrm{~s} / \mathrm{sat}$ \\
\hline Saponite-Mg & $8.3295 \mathrm{~s} / \mathrm{sat}$ & Pyrophyllite & $0.8723 \mathrm{~s} / \mathrm{sat}$ \\
\hline Saponite-K & $8.2085 \mathrm{~s} / \mathrm{sat}$ & Aragonite & $0.8375 \mathrm{~s} / \mathrm{sat}$ \\
\hline Stilbite & $7.7194 \mathrm{~s} / \mathrm{sat}$ & Diopside & $0.7340 \mathrm{~s} / \mathrm{sat}$ \\
\hline Talc & $7.2944 \mathrm{~s} / \mathrm{sat}$ & Diaspore & $\odot .7236 \mathrm{~s} / \mathrm{sat}$ \\
\hline Herschelite & $7.1633 \mathrm{~s} / \mathrm{sat}$ & Beidellite-H & $\odot .4529 \mathrm{~s} / \mathrm{sat}$ \\
\hline Saponite-H & $6.8781 \mathrm{~s} / \mathrm{sat}$ & Quartz & $\odot .3954 \mathrm{~s} / \mathrm{sat}$ \\
\hline Mesolite & $6.3674 \mathrm{~s} / \mathrm{sat}$ & Jadeite & $0.3113 \mathrm{~s} / \mathrm{sat}$ \\
\hline Celadonite & $6.3266 \mathrm{~s} / \mathrm{sat}$ & Boehmite & $0.3112 \mathrm{~s} / \mathrm{sat}$ \\
\hline Amesite-14A & $5.8544 \mathrm{~s} / \mathrm{sat}$ & Tridymite & $0.1978 \mathrm{~s} / \mathrm{sat}$ \\
\hline Clinochlore-7A & $5.6441 \mathrm{~s} / \mathrm{sat}$ & Monohydrocalcite & $\odot .1613 \mathrm{~s} / \mathrm{sat}$ \\
\hline Muscovite & $5.2634 \mathrm{~s} / \mathrm{sat}$ & Gibbsite & $0.1516 \mathrm{~s} / \mathrm{sat}$ \\
\hline Chrysotile & $4.5031 \mathrm{~s} / \mathrm{sat}$ & Magnesite & $0.1004 \mathrm{~s} / \mathrm{sat}$ \\
\hline Anthophyllite & 4.0695s/sat & Ice & -0.1194 \\
\hline Scolecite & $3.7713 \mathrm{~s} / \mathrm{sat}$ & Cristobalite(alp & -0.1666 \\
\hline Illite & $3.6590 \mathrm{~s} / \mathrm{sat}$ & Chalcedony & -0.1805 \\
\hline Montmor-Na & $3.6370 \mathrm{~s} / \mathrm{sat}$ & Dawsonite & -0.2537 \\
\hline Witherite & $3.4878 \mathrm{~s} / \mathrm{sat}$ & Huntite & -0.3245 \\
\hline Montmor-Mg & $3.4205 \mathrm{~s} / \mathrm{sat}$ & Coesite & -0.4278 \\
\hline Montmor-Ca & $3.3833 \mathrm{~s} / \mathrm{sat}$ & Cristobalite(bet & -0.6247 \\
\hline Maximum_Microcli & $3.3522 \mathrm{~s} / \mathrm{sat}$ & Kalsilite & -0.6708 \\
\hline K-Feldspar & 3.3511s/sat & Enstatite & -0.7157 \\
\hline Paragonite & $3.3323 \mathrm{~s} / \mathrm{sat}$ & Si02(am) & -0.9327 \\
\hline Montmor-K & $3.3006 \mathrm{~s} / \mathrm{sat}$ & Clinozoisite & -1.2091 \\
\hline Dolomite & $2.7425 \mathrm{~s} / \mathrm{sat}$ & Analcime & -1.2165 \\
\hline Dolomite-ord & $2.7425 \mathrm{~s} / \mathrm{sat}$ & Analcime-K & -1.2362 \\
\hline Zincite & $2.6170 \mathrm{~s} / \mathrm{sat}$ & Zoisite & -1.2550 \\
\hline Natrolite & $2.5269 \mathrm{~s} / \mathrm{sat}$ & Whitlockite & -1.4836 \\
\hline Laumontite & $2.3397 \mathrm{~s} / \mathrm{sat}$ & Nepheline & -1.5502 \\
\hline Albite_low & $2.3307 \mathrm{~s} / \mathrm{sat}$ & Barite & -1.6772 \\
\hline Albite & $2.3306 \mathrm{~s} / \mathrm{sat}$ & Fluorite & -1.8987 \\
\hline $\mathrm{Zn}(\mathrm{OH}) 2($ gamma $)$ & $2.2123 \mathrm{~s} / \mathrm{sat}$ & Brucite & -2.0705 \\
\hline $\mathrm{Zn}(\mathrm{OH}) 2$ (epsilon) & $2.1869 \mathrm{~s} / \mathrm{sat}$ & Wairakite & -2.2307 \\
\hline Beidellite-Na & $2.1237 \mathrm{~s} / \mathrm{sat}$ & Margarite & -2.2374 \\
\hline Sanidine_high & $2.1192 \mathrm{~s} / \mathrm{sat}$ & Corundum & -2.6518 \\
\hline Beidellite-Ca & 1.9379s/sat & Wollastonite & -2.7511 \\
\hline Hydroxylapatite & $1.9322 \mathrm{~s} / \mathrm{sat}$ & Okenite & -2.9078 \\
\hline $\mathrm{Zn}(\mathrm{OH}) 2($ beta $)$ & $1.9110 \mathrm{~s} / \mathrm{sat}$ & Nesquehonite & -2.9182 \\
\hline Beidellite-Mg & $1.9037 \mathrm{~s} / \mathrm{sat}$ & Artinite & -2.9587 \\
\hline $\begin{array}{l}\text { Beidellite-K } \\
\text { (only minerals }\end{array}$ & $\begin{array}{r}1.7834 \mathrm{~s} / \mathrm{sat} \\
\text { ith } \log \mathrm{Q} / \mathrm{K}>\end{array}$ & $\begin{array}{l}\text { Pseudowollastoni } \\
-3 \text { listed) }\end{array}$ & -2.9992 \\
\hline Gases & fugacity & $\log$ fug. & \\
\hline \multicolumn{4}{|c|}{ - - - - - - - - - - - - - - - - - - - } \\
\hline $02(g)$ & 0.1751 & $-\odot .757$ & \\
\hline $\mathrm{H} 20(\mathrm{~g})$ & $\odot .01903$ & -1.721 & \\
\hline
\end{tabular}




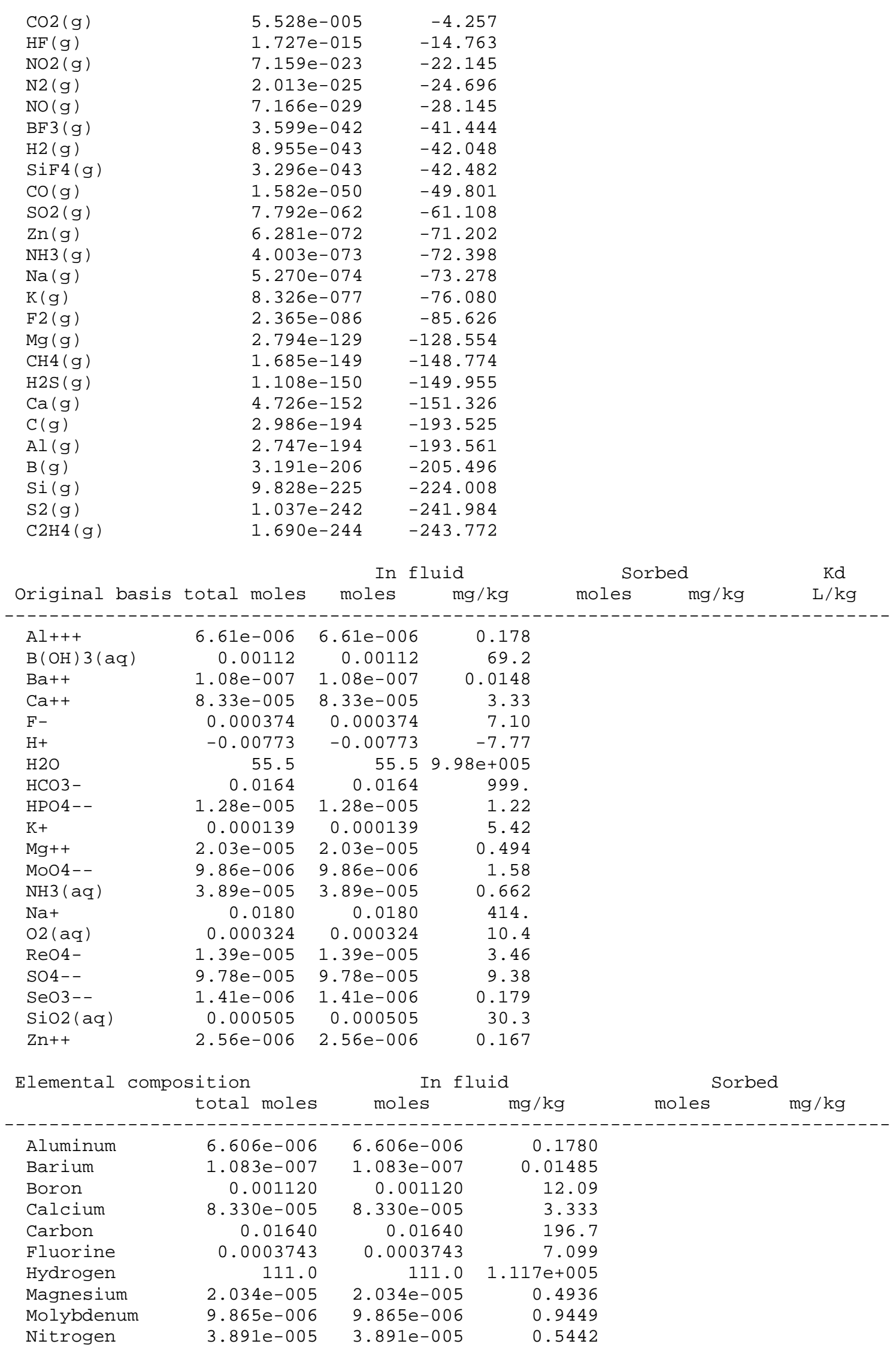




Oxygen
Phosphorus
Potassium
Rhenium
Selenium
Silicon
Sodium
Sulfur
Zinc

oxygen

Potassium

Selenium

Zinc

$\begin{array}{rr}55.56 & 8.876 \mathrm{e}+005 \\ 1.275 \mathrm{e}-005 & 0.3944 \\ 0.0001388 & 5.418 \\ 1.386 \mathrm{e}-005 & 2.576 \\ 1.409 \mathrm{e}-006 & 0.1111 \\ 0.0005049 & 14.16 \\ 0.01803 & 414.0 \\ 9.780 \mathrm{e}-005 & 3.131 \\ 2.560 \mathrm{e}-006 & 0.1672\end{array}$

55.56

$\odot .0001388$

$1.386 \mathrm{e}-005$

1. $409 \mathrm{e}-006$

.0005049

0.01803

$9.780 \mathrm{e}-005$

2. 560e-006

2. $560 \mathrm{e}-006$

$\odot .1672$ 


\section{Sample D10END}

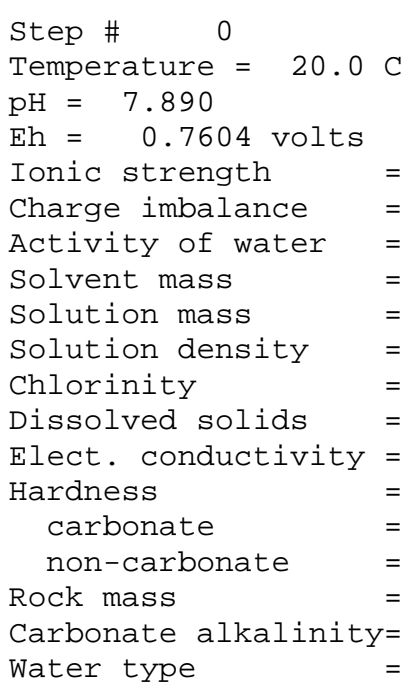

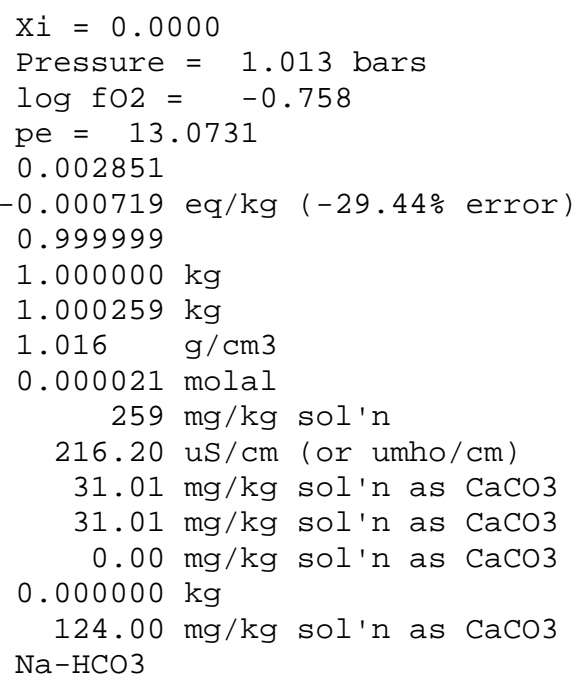

No minerals in system.

\begin{tabular}{|c|c|c|c|c|}
\hline Aqueous species & molality & $\mathrm{mg} / \mathrm{kg}$ sol'n & act. coef. & $\log$ act \\
\hline$\cdots$ & 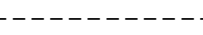 & - - - - - - - - - & 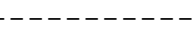 & - \\
\hline HCO3 - & $\odot .0 \odot 2446$ & 149.2 & $\odot .9437$ & -2.6368 \\
\hline $\mathrm{Na}+$ & $\odot .001416$ & 32.55 & 0.9437 & -2.8741 \\
\hline SiO2(aq) & $\odot .0 \odot \odot 4725$ & 28.38 & 1.0000 & -3.3256 \\
\hline $\mathrm{Ca}++$ & ๑. 0002593 & 10.39 & 0.7985 & -3.6839 \\
\hline $02(\mathrm{aq})$ & $\odot .0 \odot \odot 2449$ & 7.835 & 1.0000 & -3.6110 \\
\hline SO4- - & $9.606 e-005$ & 9.226 & 0.7926 & -4.1184 \\
\hline F- & $8.118 \mathrm{e}-\odot \odot 5$ & 1.542 & $\odot .9433$ & -4.1159 \\
\hline $\mathrm{co2}(\mathrm{aq})$ & $7.252 \mathrm{e}-\odot \odot 5$ & 3.191 & $1.000 \odot$ & -4.1395 \\
\hline $\mathrm{K}+$ & $5.962 \mathrm{e}-0 \odot 5$ & 2.330 & 0.9428 & -4.2502 \\
\hline $\mathrm{Mg}++$ & $3.813 e-005$ & 0.9266 & 0.8040 & -4.5134 \\
\hline Cl- & $2.142 \mathrm{e}-0 \odot 5$ & 0.7593 & 0.9428 & -4.6947 \\
\hline N03 - & 1. $455 \mathrm{e}-\odot \odot 5$ & $\odot .9022$ & $\odot .9428$ & -4.8626 \\
\hline CO3-- & $9.320 \mathrm{e}-\odot \odot 6$ & $\odot .5591$ & $\odot .7941$ & -5.1307 \\
\hline Re04 - & $9.302 e-006$ & 2.327 & 0.9437 & -5.0566 \\
\hline Al02- & $8.126 e-0 \odot 6$ & 0.4791 & $\odot .9437$ & -5.1153 \\
\hline CaHCO3+ & $5.750 \mathrm{e}-006$ & 0.5811 & $\odot .9437$ & -5.2655 \\
\hline $\operatorname{NaHCO} 3(\mathrm{aq})$ & $4.865 e-006$ & 0.4086 & 1.0000 & -5.3129 \\
\hline $\mathrm{Fe}(\mathrm{OH}) 3(\mathrm{aq})$ & 4. $494 \mathrm{e}-\odot \odot 6$ & $\odot .4801$ & $1.00 \odot \odot$ & -5.3474 \\
\hline HSi03- & $3.808 e-006$ & $\odot .2935$ & 0.9437 & -5.4444 \\
\hline Mo04-- & $3.293 e-0 \odot 6$ & 0.5265 & 0.7941 & -5.5826 \\
\hline CaC03( aq) & $2.958 \mathrm{e}-\odot \odot 6$ & $\odot .2960$ & $1.00 \odot \odot$ & -5.5290 \\
\hline $\mathrm{Zn}++$ & $2.723 \mathrm{e}-\odot \odot 6$ & 0.1780 & 0.7985 & -5.6626 \\
\hline CaS04(aq) & $1.993 e-006$ & 0.2713 & 1.0000 & -5.7005 \\
\hline $\mathrm{MgHCO} 3+$ & $8.314 \mathrm{e}-0 \odot 7$ & ๑.07091 & 0.9437 & -6.1054 \\
\hline $\mathrm{OH}-$ & $5.513 e-0 \odot 7$ & 0.009374 & 0.9433 & -6.2840 \\
\hline $\operatorname{MgS0} 4(\mathrm{aq})$ & $5.315 e-\odot \odot 7$ & $\odot .06396$ & $1.00 \odot \odot$ & -6.2745 \\
\hline HAl02 ( aq) & $3.662 \mathrm{e}-0 \odot 7$ & ๑. . 02196 & 1.0000 & -6.4362 \\
\hline NaHSiO3(aq) & $2.399 \mathrm{e}-0 \odot 7$ & $\odot .0240 \odot$ & 1.0000 & -6.6200 \\
\hline $\operatorname{MgC03}(\mathrm{aq})$ & $2.070 \mathrm{e}-0 \odot 7$ & 0.01745 & 1.0000 & -6.6841 \\
\hline $\mathrm{Ba}++$ & $8.793 e-\odot \odot 8$ & ๑. .01207 & $\odot .7956$ & -7.1551 \\
\hline $\mathrm{CaF}+$ & $7.955 \mathrm{e}-\odot \odot 8$ & $\odot .004698$ & $\odot .9437$ & -7.1245 \\
\hline $\mathrm{MgF}+$ & $5.687 e-0 \odot 8$ & 0.002462 & $\odot .9437$ & -7.2703 \\
\hline $\mathrm{NaCO} 3-$ & 3. 956e-๑०8 & 0.003283 & 0.9437 & -7.4278 \\
\hline KSO4 - & $3.447 \mathrm{e}-\odot \odot 8$ & $\odot .0 \odot 4657$ & $\odot .9437$ & -7.4878 \\
\hline ZnSO4(aq) & 3. $065 e-008$ & ๑. .0०4947 & 1.0000 & -7.5136 \\
\hline $\mathrm{H}+$ & $1.359 e-008$ & $1.369 e-005$ & 0.9479 & -7.8900 \\
\hline
\end{tabular}




\begin{tabular}{|c|c|c|c|}
\hline Mineral saturation & \multicolumn{2}{|l|}{$\log Q / K$} & $\log \mathrm{Q} / \mathrm{K}$ \\
\hline \\
\hline Nontronite-Ca & $18.1334 \mathrm{~s} / \mathrm{sat}$ & Natrolite & $1.9267 \mathrm{~s} / \mathrm{sat}$ \\
\hline Nontronite-Mg & $18.0252 \mathrm{~s} / \mathrm{sat}$ & Smectite-low-Fe- & $1.7830 \mathrm{~s} / \mathrm{sat}$ \\
\hline Nontronite-Na & $17.7569 \mathrm{~s} / \mathrm{sat}$ & Corundum & $1.7747 \mathrm{~s} / \mathrm{sat}$ \\
\hline Nontronite-K & $17.6566 \mathrm{~s} / \mathrm{sat}$ & Prehnite & $1.2983 \mathrm{~s} / \mathrm{sat}$ \\
\hline Nontronite-H & $17.1187 \mathrm{~s} / \mathrm{sat}$ & Albite_high & $1.2945 \mathrm{~s} / \mathrm{sat}$ \\
\hline Clinoptilolite-h & 16.0169s/sat & Kyanite & $1.1962 \mathrm{~s} / \mathrm{sat}$ \\
\hline Clinoptilolite-C & $15.9879 \mathrm{~s} / \mathrm{sat}$ & Witherite & $1.1489 \mathrm{~s} / \mathrm{sat}$ \\
\hline Hematite & $12.8136 \mathrm{~s} / \mathrm{sat}$ & Wairakite & ๑.9881s/sat \\
\hline Clinoptilolite-h & $12.7718 \mathrm{~s} / \mathrm{sat}$ & Clinozoisite & $0.9611 \mathrm{~s} / \mathrm{sat}$ \\
\hline Clinoptilolite-N & $12.7709 \mathrm{~s} / \mathrm{sat}$ & Magnetite & $0.9605 \mathrm{~s} / \mathrm{sat}$ \\
\hline Clinoptilolite-K & $12.0126 \mathrm{~s} / \mathrm{sat}$ & Zoisite & $0.9152 \mathrm{~s} / \mathrm{sat}$ \\
\hline Stilbite & 12.0010s/sat & Andalusite & ๑.9127s/sat \\
\hline Clinoptilolite-h & $11.9028 \mathrm{~s} / \mathrm{sat}$ & Quartz & $0.8062 \mathrm{~s} / \mathrm{sat}$ \\
\hline Ferrite-Zn & $11.0101 \mathrm{~s} / \mathrm{sat}$ & Tridymite & $\odot .6086 \mathrm{~s} / \mathrm{sat}$ \\
\hline Muscovite & $10.7338 \mathrm{~s} / \mathrm{sat}$ & Sillimanite & $0.5392 \mathrm{~s} / \mathrm{sat}$ \\
\hline Beidellite-Ca & $8.1321 \mathrm{~s} / \mathrm{sat}$ & Dawsonite & $0.3459 \mathrm{~s} / \mathrm{sat}$ \\
\hline Paragonite & $8.0750 \mathrm{~s} / \mathrm{sat}$ & Cristobalite(alp & $\odot .2442 \mathrm{~s} / \mathrm{sat}$ \\
\hline Beidellite-Mg & $8.0238 \mathrm{~s} / \mathrm{sat}$ & Saponite-Ca & $0.2314 \mathrm{~s} / \mathrm{sat}$ \\
\hline Mesolite & $8.0197 \mathrm{~s} / \mathrm{sat}$ & Chalcedony & $0.2303 \mathrm{~s} / \mathrm{sat}$ \\
\hline Illite & $7.9213 \mathrm{~s} / \mathrm{sat}$ & Jadeite & $\odot .2167 \mathrm{~s} / \mathrm{sat}$ \\
\hline Beidellite-Na & $7.7555 \mathrm{~s} / \mathrm{sat}$ & Saponite-Mg & $0.1238 \mathrm{~s} / \mathrm{sat}$ \\
\hline Epidote-ord & $7.7304 \mathrm{~s} / \mathrm{sat}$ & Coesite & -0.0170 \\
\hline Epidote & $7.7299 \mathrm{~s} / \mathrm{sat}$ & Ice & -0.1194 \\
\hline Beidellite-K & $7.6554 \mathrm{~s} / \mathrm{sat}$ & Saponite-Na & -0.1451 \\
\hline Herschelite & $7.5377 \mathrm{~s} / \mathrm{sat}$ & Cristobalite (bet & -0.2138 \\
\hline Montmor-Ca & $7.1633 \mathrm{~s} / \mathrm{sat}$ & Saponite-K & -0.2453 \\
\hline Montmor-Mg & $7.1266 \mathrm{~s} / \mathrm{sat}$ & Calcite & -0.3529 \\
\hline Beidellite-H & $7.1175 \mathrm{~s} / \mathrm{sat}$ & Dolomite & -0.3763 \\
\hline Kaolinite & 7.0059s/sat & Dolomite-ord & -0.3763 \\
\hline Pyrophyllite & $6.9420 \mathrm{~s} / \mathrm{sat}$ & Phlogopite & -0.3888 \\
\hline Montmor-Na & $6.8547 \mathrm{~s} / \mathrm{sat}$ & Kalsilite & -0.4485 \\
\hline Montmor-K & $6.7585 \mathrm{~s} / \mathrm{sat}$ & Aragonite & -0.4975 \\
\hline Scolecite & $6.5793 \mathrm{~s} / \mathrm{sat}$ & Si02(am) & -0.5219 \\
\hline Goethite & $5.9323 \mathrm{~s} / \mathrm{sat}$ & Analcime-K & -0.5791 \\
\hline Laumontite & $5.5586 \mathrm{~s} / \mathrm{sat}$ & Saponite-H & -0.7832 \\
\hline Andradite & $5.4714 \mathrm{~s} / \mathrm{sat}$ & Smithsonite & -0.9602 \\
\hline Margarite & $4.5863 \mathrm{~s} / \mathrm{sat}$ & Talc & -0.9617 \\
\hline Celadonite & $4.4815 \mathrm{~s} / \mathrm{sat}$ & Anorthite & -1.1246 \\
\hline Maximum_Microcli & 4.3962s/sat & Monohydrocalcite & -1.1737 \\
\hline K-Feldspar & 4.3950s/sat & Barite & -1.2071 \\
\hline Mordenite & $3.7007 \mathrm{~s} / \mathrm{sat}$ & Analcime & -1.2581 \\
\hline Lawsonite & 3.4164s/sat & Smectite-high-Fe & -1.3049 \\
\hline Sanidine_high & $3.1631 \mathrm{~s} / \mathrm{sat}$ & Zincite & -1.3611 \\
\hline Ferrite-Ca & $3.0842 \mathrm{~s} / \mathrm{sat}$ & Magnesite & -1.6834 \\
\hline Diaspore & $2.9368 \mathrm{~s} / \mathrm{sat}$ & $\mathrm{Zn}(\mathrm{OH}) 2($ gamma $)$ & -1.7658 \\
\hline Zn2SiO4 & $2.6941 \mathrm{~s} / \mathrm{sat}$ & Clinochlore-14A & -1.7810 \\
\hline Ferrite-Mg & $2.6770 \mathrm{~s} / \mathrm{sat}$ & $\mathrm{Zn}(\mathrm{OH}) 2$ (epsilon) & -1.7911 \\
\hline Albite_low & $2.6468 \mathrm{~s} / \mathrm{sat}$ & Fluorite & -1.8288 \\
\hline Albite & $2.6467 \mathrm{~s} / \mathrm{sat}$ & Dolomite-dis & -1.9581 \\
\hline Boehmite & $2.5244 \mathrm{~s} / \mathrm{sat}$ & Nepheline & -2.0557 \\
\hline Gibbsite & $2.3648 \mathrm{~s} / \mathrm{sat}$ & $\mathrm{Zn}(\mathrm{OH}) 2($ beta $)$ & -2.0670 \\
\hline Amesite-14A & $2.3298 \mathrm{~s} / \mathrm{sat}$ & $\mathrm{Fe}(\mathrm{OH}) 3$ & -2.7556 \\
\hline (only minerals & ith $\log Q / K>$ & 3 listed) & \\
\hline Gases & fugacity & $\log$ fug. & \\
\hline - - - - - - - - - - - - & 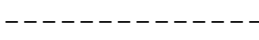 & 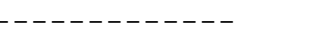 & \\
\hline $02(g)$ & 0.1747 & $-\odot .758$ & \\
\hline $\mathrm{H} 2 \mathrm{O}(\mathrm{g})$ & ๑. 01903 & -1.721 & \\
\hline $\operatorname{co2}(\mathrm{g})$ & $\odot .001814$ & -2.741 & \\
\hline
\end{tabular}




\begin{tabular}{|c|c|c|}
\hline $\mathrm{HF}(\mathrm{g})$ & $4.987 e-014$ & $-13 \cdot 302$ \\
\hline $\mathrm{HCl}(\mathrm{g})$ & $7.724 \mathrm{e}-020$ & -19.112 \\
\hline NO2 (g) & $3.573 e-021$ & -20.447 \\
\hline N2 $(g)$ & $5.038 e-022$ & -21.298 \\
\hline NO $(g)$ & $3.580 \mathrm{e}-027$ & -26.446 \\
\hline Cl2(g) & $7.331 e-031$ & -30.135 \\
\hline $\operatorname{SiF} 4(g)$ & $5.897 e-037$ & -36.229 \\
\hline $\mathrm{H} 2(\mathrm{~g})$ & $8.966 e-043$ & -42.047 \\
\hline $\operatorname{co}(g)$ & $5.195 \mathrm{e}-\odot 49$ & -48.284 \\
\hline So2(g) & $1.649 \mathrm{e}-057$ & -56.783 \\
\hline $\mathrm{NH3}(\mathrm{g})$ & $2.006 e-071$ & -70.698 \\
\hline $\mathrm{Zn}(\mathrm{g})$ & $6.614 \mathrm{e}-076$ & -75.180 \\
\hline $\mathrm{Na}(\mathrm{g})$ & $3.913 e-077$ & -76.407 \\
\hline $\mathrm{K}(\mathrm{g})$ & $3.303 e-079$ & -78.481 \\
\hline $\mathrm{F} 2(\mathrm{~g})$ & $1.969 \mathrm{e}-\odot 83$ & -82.706 \\
\hline$M g(g)$ & 1. $403 e-132$ & -131.853 \\
\hline $\mathrm{H} 2 \mathrm{~S}(\mathrm{~g})$ & $2.353 e-146$ & -145.628 \\
\hline $\mathrm{CH} 4(\mathrm{~g})$ & $5.553 e-148$ & -147.255 \\
\hline $\mathrm{Ca}(\mathrm{g})$ & $6.669 e-155$ & -154.176 \\
\hline $\mathrm{Al}(\mathrm{g})$ & $4.496 e-192$ & -191.347 \\
\hline$C(g)$ & $9.820 \mathrm{e}-193$ & -192.008 \\
\hline $\operatorname{si}(g)$ & $2.537 e-224$ & -223.596 \\
\hline$S 2(g)$ & $4.663 e-234$ & -233.331 \\
\hline $\mathrm{C} 2 \mathrm{H} 4(\mathrm{~g})$ & $1.832 e-241$ & -240.737 \\
\hline
\end{tabular}

\begin{tabular}{|c|c|c|c|c|c|}
\hline \multirow{2}{*}{\multicolumn{2}{|c|}{ Original basis total moles }} & \multicolumn{2}{|c|}{ In fluid } & Sorbed & \multirow{2}{*}{$\begin{array}{c}\mathrm{Kd} \\
\mathrm{L} / \mathrm{kg}\end{array}$} \\
\hline & & moles & $\mathrm{mg} / \mathrm{kg}$ & moles $\quad \mathrm{mg} / \mathrm{kg}$ & \\
\hline & 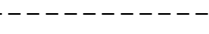 & 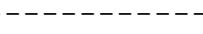 & $\cdots-\cdots$ & & \\
\hline $\mathrm{Al}+++$ & $8.50 \mathrm{e}-006$ & $8.50 \mathrm{e}-006$ & 0.229 & & \\
\hline $\mathrm{Ba}++$ & $8.81 e-008$ & $8.81 e-008$ & 0.0121 & & \\
\hline $\mathrm{Ca}++$ & 0.000270 & 0.000270 & 10.8 & & \\
\hline $\mathrm{Cl}-$ & $2.14 \mathrm{e}-\odot \odot 5$ & $2.14 \mathrm{e}-0 \odot 5$ & $\odot .760$ & & \\
\hline$F-$ & $8.13 e-005$ & $8.13 e-005$ & 1.54 & & \\
\hline $\mathrm{Fe}++$ & $4.49 e-006$ & $4.49 e-006$ & 0.251 & & \\
\hline $\mathrm{H}+$ & $-1.75 e-006$ & $-1.75 e-0 \odot 6$ & -0.00176 & & \\
\hline $\mathrm{H} 2 \mathrm{O}$ & 55.5 & 55.5 & $1.00 \mathrm{e}+006$ & & \\
\hline HCO3- & $\odot . \odot \odot 254$ & $\odot .0 \odot 254$ & 155 & & \\
\hline $\mathrm{K}+$ & $5.97 e-005$ & $5.97 e-005$ & 2.33 & & \\
\hline $\mathrm{Mg}++$ & $3.98 e-005$ & $3.98 e-005$ & $\odot .966$ & & \\
\hline Mo04 - - & $3.29 e-006$ & $3.29 e-0 \odot 6$ & 0.526 & & \\
\hline $\mathrm{NH3}(\mathrm{aq})$ & $1.46 e-005$ & $1.46 e-005$ & $\odot .248$ & & \\
\hline $\mathrm{Na}+$ & 0.00142 & ๑. 00142 & 32.7 & & \\
\hline $02(\mathrm{aq})$ & ๑. 000275 & $\odot .000275$ & 8.80 & & \\
\hline Re04 - & $9.30 \mathrm{e}-0 \odot 6$ & $9.30 \mathrm{e}-006$ & 2.33 & & \\
\hline S04 - - & $9.87 e-005$ & $9.87 e-0 \odot 5$ & 9.47 & & \\
\hline Si02(aq) & $\odot .000477$ & $\odot .000477$ & 28.6 & & \\
\hline $\mathrm{Zn++}$ & $2.75 e-006$ & $2.75 e-006$ & 0.180 & & \\
\hline
\end{tabular}

Elemental composition

In fluid

Sorbed total moles moles mg/kg moles $\mathrm{mg} / \mathrm{kg}$

\begin{tabular}{|c|c|c|c|}
\hline Aluminum & $8.498 \mathrm{e}-\odot \odot 6$ & 8. 498e-๑०6 & 0.2292 \\
\hline Barium & $8.814 \mathrm{e}-\odot \odot 8$ & 8. 814e-๑๑৪ & ๑ . 01210 \\
\hline Calcium & ๑. . $\odot \odot 2701$ & ๑. $00 \odot 2701$ & 10.82 \\
\hline Carbon & $\odot .0 \odot 2542$ & ๑..$\odot 2542$ & 30.52 \\
\hline Chlorine & $2.143 e-0 \odot 5$ & $2.143 e-0 \odot 5$ & $\odot .7595$ \\
\hline Fluorine & $8.133 e-005$ & $8.133 e-0 \odot 5$ & 1.545 \\
\hline Hydrogen & 111.0 & 111.0 & $1.119 \mathrm{e}+\odot \odot 5$ \\
\hline Iron & $4.494 \mathrm{e}-006$ & $4.494 \mathrm{e}-0 \odot 6$ & ๑. 2509 \\
\hline Magnesium & $3.976 \mathrm{e}-005$ & $3.976 \mathrm{e}-005$ & 0.9662 \\
\hline Molybdenum & $3.293 e-0 \odot 6$ & $3.293 e-\odot \odot 6$ & ๑. 3158 \\
\hline Nitrogen & 1. $455 \mathrm{e}-005$ & 1. $455 \mathrm{e}-0 \odot 5$ & 0.2038 \\
\hline oxygen & 55.52 & 55.52 & $8.880 e+005$ \\
\hline
\end{tabular}




$\begin{array}{lrrr}\text { Potassium } & 5.965 \mathrm{e}-\odot \odot 5 & 5.965 \mathrm{e}-005 & 2.332 \\ \text { Rhenium } & 9.302 \mathrm{e}-006 & 9.302 \mathrm{e}-006 & 1.732 \\ \text { Silicon } & 0.0004765 & 0.0004765 & 13.38 \\ \text { Sodium } & 0.001421 & 0.001421 & 32.66 \\ \text { Sulfur } & 9.865 \mathrm{e}-005 & 9.865 \mathrm{e}-005 & 3.163 \\ \text { Zinc } & 2.754 \mathrm{e}-006 & 2.754 \mathrm{e}-006 & 0.1800\end{array}$




\section{Sample D10ETOP}

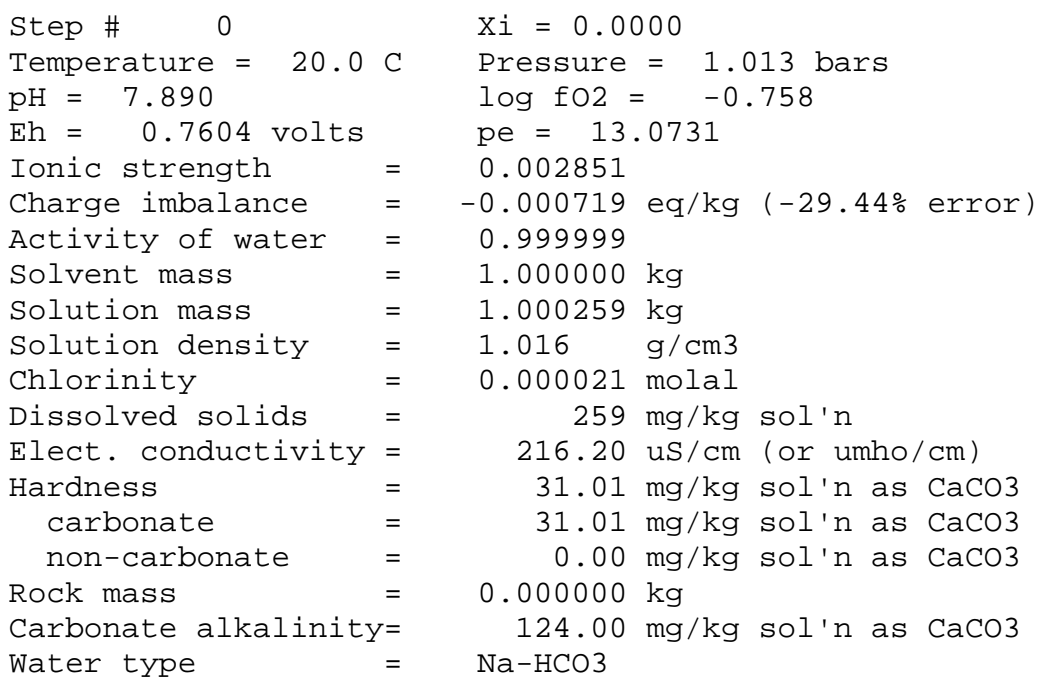

No minerals in system.

\begin{tabular}{|c|c|c|c|c|}
\hline Aqueous species & molality & $\mathrm{mg} / \mathrm{kg}$ sol'n & act. coef. & $\log a c t$ \\
\hline$-n_{-1}$ & & & $-\ldots$ & 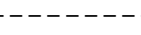 \\
\hline HCO3 - & $\odot .0 \odot 2446$ & 149.2 & $\odot .9437$ & -2.6368 \\
\hline $\mathrm{Na}+$ & $\odot .001416$ & 32.55 & $\odot .9437$ & -2.8741 \\
\hline $\operatorname{Si02}(\mathrm{aq})$ & 0.0004725 & 28.38 & 1.0000 & -3.3256 \\
\hline $\mathrm{Ca}++$ & ๑. . $\odot \odot 2593$ & 10.39 & $\odot .7985$ & -3.6839 \\
\hline $02(\mathrm{aq})$ & $\odot .0 \odot \odot 2449$ & 7.835 & $1.00 \odot \odot$ & -3.6110 \\
\hline SO4 - - & $9.606 e-005$ & 9.226 & 0.7926 & -4.1184 \\
\hline F- & $8.118 e-0 \odot 5$ & 1.542 & 0.9433 & -4.1159 \\
\hline $\mathrm{co2}(\mathrm{aq})$ & $7.252 e-005$ & 3.191 & $1.000 \odot$ & -4.1395 \\
\hline $\mathrm{K}+$ & $5.962 \mathrm{e}-005$ & 2.330 & 0.9428 & -4.2502 \\
\hline $\mathrm{Mg}++$ & $3.813 e-005$ & $\odot .9266$ & 0.8040 & -4.5134 \\
\hline Cl- & $2.142 \mathrm{e}-0 \odot 5$ & 0.7593 & 0.9428 & -4.6947 \\
\hline N03 - & 1. $455 \mathrm{e}-0 \odot 5$ & $\odot .9022$ & $\odot .9428$ & -4.8626 \\
\hline CO3-- & $9.320 \mathrm{e}-\odot \odot 6$ & $\odot .5591$ & 0.7941 & -5.1307 \\
\hline Re04 - & $9.302 \mathrm{e}-006$ & 2.327 & $\odot .9437$ & -5.0566 \\
\hline Al02- & $8.126 \mathrm{e}-0 \odot 6$ & 0.4791 & $\odot .9437$ & -5.1153 \\
\hline $\mathrm{CaHCO}+$ & $5.750 \mathrm{e}-0 \odot 6$ & 0.5811 & $\odot .9437$ & -5.2655 \\
\hline $\operatorname{NaHCO} 3(\mathrm{aq})$ & $4.865 e-006$ & 0.4086 & 1.0000 & -5.3129 \\
\hline $\mathrm{Fe}(\mathrm{OH}) 3(\mathrm{aq})$ & 4. 494e- -๑६ & ๑. 4801 & $1.0 \odot \odot \odot$ & -5.3474 \\
\hline HSiO3- & $3.808 \mathrm{e}-\odot \odot 6$ & $\odot .2935$ & 0.9437 & -5.4444 \\
\hline Mo04 - - & $3.293 e-0 \odot 6$ & 0.5265 & 0.7941 & -5.5826 \\
\hline $\mathrm{CaC0} 3(\mathrm{aq})$ & $2.958 \mathrm{e}-006$ & $\odot .2960$ & 1.0000 & -5.5290 \\
\hline $\mathrm{Zn++}$ & $2.723 \mathrm{e}-0 \odot 6$ & 0.1780 & 0.7985 & -5.6626 \\
\hline CaSo4(aq) & 1. $993 \mathrm{e}-\odot \odot 6$ & $\odot .2713$ & $1.00 \odot \odot$ & -5.7005 \\
\hline $\mathrm{MgHCO} 3+$ & 8. 314e- -๑७ & ๑. 07091 & $\odot .9437$ & -6.1054 \\
\hline $\mathrm{OH}-$ & $5.513 e-0 \odot 7$ & 0.009374 & 0.9433 & -6.2840 \\
\hline $\operatorname{MgS0} 4(\mathrm{aq})$ & $5.315 e-\odot \odot 7$ & ๑. 06396 & $1.0 \odot \odot \odot$ & -6.2745 \\
\hline $\mathrm{HAlO} 2(\mathrm{aq})$ & $3.662 \mathrm{e}-0 \odot 7$ & ๑. .02196 & 1.0000 & -6.4362 \\
\hline NaHSiO3(aq) & 2. 399e- $-0 \odot 7$ & $\odot .02400$ & 1. 0000 & -6.6200 \\
\hline $\operatorname{MgC03}(a q)$ & $2.070 \mathrm{e}-\odot \odot 7$ & 0.01745 & $1.0 \odot \odot \odot$ & -6.6841 \\
\hline $\mathrm{Ba}++$ & $8.793 e-0 \odot 8$ & 0.01207 & 0.7956 & -7.1551 \\
\hline $\mathrm{CaF}+$ & $7.955 e-\odot \odot 8$ & $\odot .004698$ & 0.9437 & -7.1245 \\
\hline MgF+ & $5.687 e-\odot \odot 8$ & ๑ . $0 \odot 2462$ & $\odot .9437$ & -7.2703 \\
\hline $\mathrm{NaCO} 3-$ & 3. $956 \mathrm{e}-0 \odot 8$ & $\odot .003283$ & 0.9437 & -7.4278 \\
\hline KSO4 - & $3.447 e-\odot \odot 8$ & ๑. .๑4657 & $\odot .9437$ & -7.4878 \\
\hline $\mathrm{ZnSO} 4(\mathrm{aq})$ & $3.065 e-008$ & $\odot .004947$ & 1.0000 & -7.5136 \\
\hline $\mathrm{H}+$ & 1. $359 \mathrm{e}-\odot \odot 8$ & $1.369 e-0 \odot 5$ & $\odot .9479$ & -7.8900 \\
\hline
\end{tabular}




\begin{tabular}{|c|c|c|c|}
\hline Mineral saturation & \multicolumn{2}{|l|}{$\log Q / K$} & $\log \mathrm{Q} / \mathrm{K}$ \\
\hline \\
\hline Nontronite-Ca & $18.1334 \mathrm{~s} / \mathrm{sat}$ & Natrolite & $1.9267 \mathrm{~s} / \mathrm{sat}$ \\
\hline Nontronite-Mg & $18.0252 \mathrm{~s} / \mathrm{sat}$ & Smectite-low-Fe- & $1.7830 \mathrm{~s} / \mathrm{sat}$ \\
\hline Nontronite-Na & $17.7569 \mathrm{~s} / \mathrm{sat}$ & Corundum & $1.7747 \mathrm{~s} / \mathrm{sat}$ \\
\hline Nontronite-K & $17.6566 \mathrm{~s} / \mathrm{sat}$ & Prehnite & $1.2983 \mathrm{~s} / \mathrm{sat}$ \\
\hline Nontronite-H & $17.1187 \mathrm{~s} / \mathrm{sat}$ & Albite_high & $1.2945 \mathrm{~s} / \mathrm{sat}$ \\
\hline Clinoptilolite-h & 16.0169s/sat & Kyanite & $1.1962 \mathrm{~s} / \mathrm{sat}$ \\
\hline Clinoptilolite-C & $15.9879 \mathrm{~s} / \mathrm{sat}$ & Witherite & $1.1489 \mathrm{~s} / \mathrm{sat}$ \\
\hline Hematite & $12.8136 \mathrm{~s} / \mathrm{sat}$ & Wairakite & ๑.9881s/sat \\
\hline Clinoptilolite-h & $12.7718 \mathrm{~s} / \mathrm{sat}$ & Clinozoisite & $0.9611 \mathrm{~s} / \mathrm{sat}$ \\
\hline Clinoptilolite-N & $12.7709 \mathrm{~s} / \mathrm{sat}$ & Magnetite & $0.9605 \mathrm{~s} / \mathrm{sat}$ \\
\hline Clinoptilolite-K & $12.0126 \mathrm{~s} / \mathrm{sat}$ & Zoisite & $0.9152 \mathrm{~s} / \mathrm{sat}$ \\
\hline Stilbite & 12.0010s/sat & Andalusite & ๑.9127s/sat \\
\hline Clinoptilolite-h & $11.9028 \mathrm{~s} / \mathrm{sat}$ & Quartz & $0.8062 \mathrm{~s} / \mathrm{sat}$ \\
\hline Ferrite-Zn & $11.0101 \mathrm{~s} / \mathrm{sat}$ & Tridymite & $\odot .6086 \mathrm{~s} / \mathrm{sat}$ \\
\hline Muscovite & $10.7338 \mathrm{~s} / \mathrm{sat}$ & Sillimanite & $0.5392 \mathrm{~s} / \mathrm{sat}$ \\
\hline Beidellite-Ca & $8.1321 \mathrm{~s} / \mathrm{sat}$ & Dawsonite & $0.3459 \mathrm{~s} / \mathrm{sat}$ \\
\hline Paragonite & $8.0750 \mathrm{~s} / \mathrm{sat}$ & Cristobalite(alp & $\odot .2442 \mathrm{~s} / \mathrm{sat}$ \\
\hline Beidellite-Mg & $8.0238 \mathrm{~s} / \mathrm{sat}$ & Saponite-Ca & $0.2314 \mathrm{~s} / \mathrm{sat}$ \\
\hline Mesolite & $8.0197 \mathrm{~s} / \mathrm{sat}$ & Chalcedony & $0.2303 \mathrm{~s} / \mathrm{sat}$ \\
\hline Illite & $7.9213 \mathrm{~s} / \mathrm{sat}$ & Jadeite & $\odot .2167 \mathrm{~s} / \mathrm{sat}$ \\
\hline Beidellite-Na & $7.7555 \mathrm{~s} / \mathrm{sat}$ & Saponite-Mg & $0.1238 \mathrm{~s} / \mathrm{sat}$ \\
\hline Epidote-ord & $7.7304 \mathrm{~s} / \mathrm{sat}$ & Coesite & -0.0170 \\
\hline Epidote & $7.7299 \mathrm{~s} / \mathrm{sat}$ & Ice & -0.1194 \\
\hline Beidellite-K & $7.6554 \mathrm{~s} / \mathrm{sat}$ & Saponite-Na & -0.1451 \\
\hline Herschelite & $7.5377 \mathrm{~s} / \mathrm{sat}$ & Cristobalite (bet & -0.2138 \\
\hline Montmor-Ca & $7.1633 \mathrm{~s} / \mathrm{sat}$ & Saponite-K & -0.2453 \\
\hline Montmor-Mg & $7.1266 \mathrm{~s} / \mathrm{sat}$ & Calcite & -0.3529 \\
\hline Beidellite-H & $7.1175 \mathrm{~s} / \mathrm{sat}$ & Dolomite & -0.3763 \\
\hline Kaolinite & 7.0059s/sat & Dolomite-ord & -0.3763 \\
\hline Pyrophyllite & $6.9420 \mathrm{~s} / \mathrm{sat}$ & Phlogopite & -0.3888 \\
\hline Montmor-Na & $6.8547 \mathrm{~s} / \mathrm{sat}$ & Kalsilite & -0.4485 \\
\hline Montmor-K & $6.7585 \mathrm{~s} / \mathrm{sat}$ & Aragonite & -0.4975 \\
\hline Scolecite & $6.5793 \mathrm{~s} / \mathrm{sat}$ & Si02(am) & -0.5219 \\
\hline Goethite & $5.9323 \mathrm{~s} / \mathrm{sat}$ & Analcime-K & -0.5791 \\
\hline Laumontite & $5.5586 \mathrm{~s} / \mathrm{sat}$ & Saponite-H & -0.7832 \\
\hline Andradite & $5.4714 \mathrm{~s} / \mathrm{sat}$ & Smithsonite & -0.9602 \\
\hline Margarite & $4.5863 \mathrm{~s} / \mathrm{sat}$ & Talc & -0.9617 \\
\hline Celadonite & $4.4815 \mathrm{~s} / \mathrm{sat}$ & Anorthite & -1.1246 \\
\hline Maximum_Microcli & 4.3962s/sat & Monohydrocalcite & -1.1737 \\
\hline K-Feldspar & 4.3950s/sat & Barite & -1.2071 \\
\hline Mordenite & $3.7007 \mathrm{~s} / \mathrm{sat}$ & Analcime & -1.2581 \\
\hline Lawsonite & 3.4164s/sat & Smectite-high-Fe & -1.3049 \\
\hline Sanidine_high & $3.1631 \mathrm{~s} / \mathrm{sat}$ & Zincite & -1.3611 \\
\hline Ferrite-Ca & $3.0842 \mathrm{~s} / \mathrm{sat}$ & Magnesite & -1.6834 \\
\hline Diaspore & $2.9368 \mathrm{~s} / \mathrm{sat}$ & $\mathrm{Zn}(\mathrm{OH}) 2($ gamma $)$ & -1.7658 \\
\hline Zn2SiO4 & $2.6941 \mathrm{~s} / \mathrm{sat}$ & Clinochlore-14A & -1.7810 \\
\hline Ferrite-Mg & $2.6770 \mathrm{~s} / \mathrm{sat}$ & $\mathrm{Zn}(\mathrm{OH}) 2$ (epsilon) & -1.7911 \\
\hline Albite_low & $2.6468 \mathrm{~s} / \mathrm{sat}$ & Fluorite & -1.8288 \\
\hline Albite & $2.6467 \mathrm{~s} / \mathrm{sat}$ & Dolomite-dis & -1.9581 \\
\hline Boehmite & $2.5244 \mathrm{~s} / \mathrm{sat}$ & Nepheline & -2.0557 \\
\hline Gibbsite & $2.3648 \mathrm{~s} / \mathrm{sat}$ & $\mathrm{Zn}(\mathrm{OH}) 2($ beta $)$ & -2.0670 \\
\hline Amesite-14A & $2.3298 \mathrm{~s} / \mathrm{sat}$ & $\mathrm{Fe}(\mathrm{OH}) 3$ & -2.7556 \\
\hline (only minerals & ith $\log Q / K>$ & 3 listed) & \\
\hline Gases & fugacity & $\log$ fug. & \\
\hline - - - - - - - - - - - - & 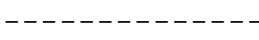 & 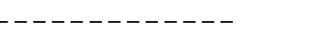 & \\
\hline $02(g)$ & 0.1747 & $-\odot .758$ & \\
\hline $\mathrm{H} 2 \mathrm{O}(\mathrm{g})$ & ๑. 01903 & -1.721 & \\
\hline $\operatorname{co2}(\mathrm{g})$ & $\odot .001814$ & -2.741 & \\
\hline
\end{tabular}




\begin{tabular}{|c|c|c|}
\hline $\mathrm{HF}(\mathrm{g})$ & $4.987 e-014$ & $-13 \cdot 302$ \\
\hline $\mathrm{HCl}(\mathrm{g})$ & $7.724 \mathrm{e}-020$ & -19.112 \\
\hline NO2 (g) & $3.573 e-021$ & -20.447 \\
\hline N2 $(g)$ & $5.038 e-022$ & -21.298 \\
\hline NO $(g)$ & $3.580 \mathrm{e}-027$ & -26.446 \\
\hline Cl2(g) & $7.331 e-031$ & -30.135 \\
\hline $\operatorname{SiF} 4(g)$ & $5.897 e-037$ & -36.229 \\
\hline $\mathrm{H} 2(\mathrm{~g})$ & $8.966 e-043$ & -42.047 \\
\hline $\operatorname{co}(g)$ & $5.195 \mathrm{e}-\odot 49$ & -48.284 \\
\hline So2(g) & $1.649 \mathrm{e}-057$ & -56.783 \\
\hline $\mathrm{NH3}(\mathrm{g})$ & $2.006 e-071$ & -70.698 \\
\hline $\mathrm{Zn}(\mathrm{g})$ & $6.614 \mathrm{e}-076$ & -75.180 \\
\hline $\mathrm{Na}(\mathrm{g})$ & $3.913 e-077$ & -76.407 \\
\hline $\mathrm{K}(\mathrm{g})$ & $3.303 e-079$ & -78.481 \\
\hline $\mathrm{F} 2(\mathrm{~g})$ & $1.969 \mathrm{e}-\odot 83$ & -82.706 \\
\hline$M g(g)$ & 1. $403 e-132$ & -131.853 \\
\hline $\mathrm{H} 2 \mathrm{~S}(\mathrm{~g})$ & $2.353 e-146$ & -145.628 \\
\hline $\mathrm{CH} 4(\mathrm{~g})$ & $5.553 e-148$ & -147.255 \\
\hline $\mathrm{Ca}(\mathrm{g})$ & $6.669 e-155$ & -154.176 \\
\hline $\mathrm{Al}(\mathrm{g})$ & $4.496 e-192$ & -191.347 \\
\hline$C(g)$ & $9.820 \mathrm{e}-193$ & -192.008 \\
\hline $\operatorname{si}(g)$ & $2.537 e-224$ & -223.596 \\
\hline$S 2(g)$ & $4.663 e-234$ & -233.331 \\
\hline $\mathrm{C} 2 \mathrm{H} 4(\mathrm{~g})$ & $1.832 e-241$ & -240.737 \\
\hline
\end{tabular}

\begin{tabular}{|c|c|c|c|c|c|}
\hline \multirow{2}{*}{\multicolumn{2}{|c|}{ Original basis total moles }} & \multicolumn{2}{|c|}{ In fluid } & Sorbed & \multirow{2}{*}{$\begin{array}{c}\mathrm{Kd} \\
\mathrm{L} / \mathrm{kg}\end{array}$} \\
\hline & & moles & $\mathrm{mg} / \mathrm{kg}$ & moles $\quad \mathrm{mg} / \mathrm{kg}$ & \\
\hline & 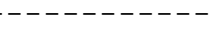 & 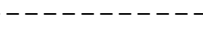 & $\cdots-\cdots$ & & \\
\hline $\mathrm{Al}+++$ & $8.50 \mathrm{e}-006$ & $8.50 \mathrm{e}-006$ & 0.229 & & \\
\hline $\mathrm{Ba}++$ & $8.81 e-008$ & $8.81 e-008$ & 0.0121 & & \\
\hline $\mathrm{Ca}++$ & 0.000270 & 0.000270 & 10.8 & & \\
\hline $\mathrm{Cl}-$ & $2.14 \mathrm{e}-\odot \odot 5$ & $2.14 \mathrm{e}-0 \odot 5$ & $\odot .760$ & & \\
\hline$F-$ & $8.13 e-005$ & $8.13 e-005$ & 1.54 & & \\
\hline $\mathrm{Fe}++$ & $4.49 e-006$ & $4.49 e-006$ & 0.251 & & \\
\hline $\mathrm{H}+$ & $-1.75 e-006$ & $-1.75 e-0 \odot 6$ & -0.00176 & & \\
\hline $\mathrm{H} 2 \mathrm{O}$ & 55.5 & 55.5 & $1.00 \mathrm{e}+006$ & & \\
\hline HCO3- & $\odot . \odot \odot 254$ & $\odot .0 \odot 254$ & 155 & & \\
\hline $\mathrm{K}+$ & $5.97 e-005$ & $5.97 e-005$ & 2.33 & & \\
\hline $\mathrm{Mg}++$ & $3.98 e-005$ & $3.98 e-005$ & $\odot .966$ & & \\
\hline Mo04 - - & $3.29 e-006$ & $3.29 e-0 \odot 6$ & 0.526 & & \\
\hline $\mathrm{NH3}(\mathrm{aq})$ & $1.46 e-005$ & $1.46 e-005$ & $\odot .248$ & & \\
\hline $\mathrm{Na}+$ & 0.00142 & ๑. 00142 & 32.7 & & \\
\hline $02(\mathrm{aq})$ & ๑. 000275 & $\odot .000275$ & 8.80 & & \\
\hline Re04 - & $9.30 \mathrm{e}-0 \odot 6$ & $9.30 \mathrm{e}-006$ & 2.33 & & \\
\hline S04 - - & $9.87 e-005$ & $9.87 e-0 \odot 5$ & 9.47 & & \\
\hline Si02(aq) & $\odot .000477$ & $\odot .000477$ & 28.6 & & \\
\hline $\mathrm{Zn++}$ & $2.75 e-006$ & $2.75 e-006$ & 0.180 & & \\
\hline
\end{tabular}

Elemental composition

In fluid

Sorbed total moles moles mg/kg moles $\mathrm{mg} / \mathrm{kg}$

\begin{tabular}{|c|c|c|c|}
\hline Aluminum & $8.498 \mathrm{e}-\odot \odot 6$ & 8. 498e-๑०6 & 0.2292 \\
\hline Barium & $8.814 \mathrm{e}-\odot \odot 8$ & 8. 814e-๑๑৪ & ๑ . 01210 \\
\hline Calcium & ๑. . $\odot \odot 2701$ & ๑. $00 \odot 2701$ & 10.82 \\
\hline Carbon & $\odot .0 \odot 2542$ & ๑..$\odot 2542$ & 30.52 \\
\hline Chlorine & $2.143 e-0 \odot 5$ & $2.143 e-0 \odot 5$ & $\odot .7595$ \\
\hline Fluorine & $8.133 e-005$ & $8.133 e-0 \odot 5$ & 1.545 \\
\hline Hydrogen & 111.0 & 111.0 & $1.119 \mathrm{e}+\odot \odot 5$ \\
\hline Iron & $4.494 \mathrm{e}-006$ & $4.494 \mathrm{e}-0 \odot 6$ & ๑. 2509 \\
\hline Magnesium & $3.976 \mathrm{e}-005$ & $3.976 \mathrm{e}-005$ & 0.9662 \\
\hline Molybdenum & $3.293 e-0 \odot 6$ & $3.293 e-\odot \odot 6$ & ๑. 3158 \\
\hline Nitrogen & 1. $455 \mathrm{e}-005$ & 1. $455 \mathrm{e}-0 \odot 5$ & 0.2038 \\
\hline oxygen & 55.52 & 55.52 & $8.880 e+005$ \\
\hline
\end{tabular}




$\begin{array}{lrrr}\text { Potassium } & 5.965 \mathrm{e}-\odot \odot 5 & 5.965 \mathrm{e}-005 & 2.332 \\ \text { Rhenium } & 9.302 \mathrm{e}-006 & 9.302 \mathrm{e}-006 & 1.732 \\ \text { Silicon } & 0.0004765 & 0.0004765 & 13.38 \\ \text { Sodium } & 0.001421 & 0.001421 & 32.66 \\ \text { Sulfur } & 9.865 \mathrm{e}-005 & 9.865 \mathrm{e}-005 & 3.163 \\ \text { Zinc } & 2.754 \mathrm{e}-006 & 2.754 \mathrm{e}-006 & 0.1800\end{array}$




\section{Sample D10NBOTg}

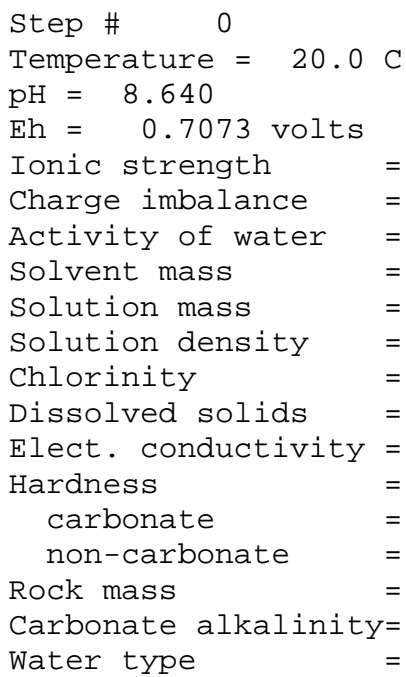

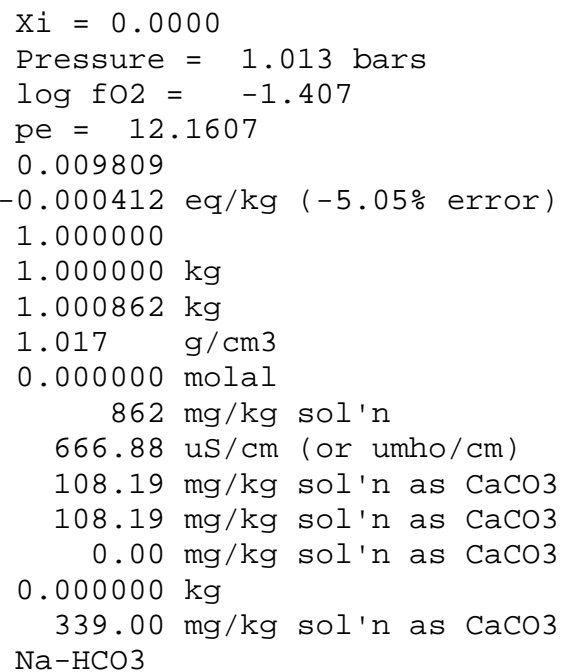

No minerals in system.

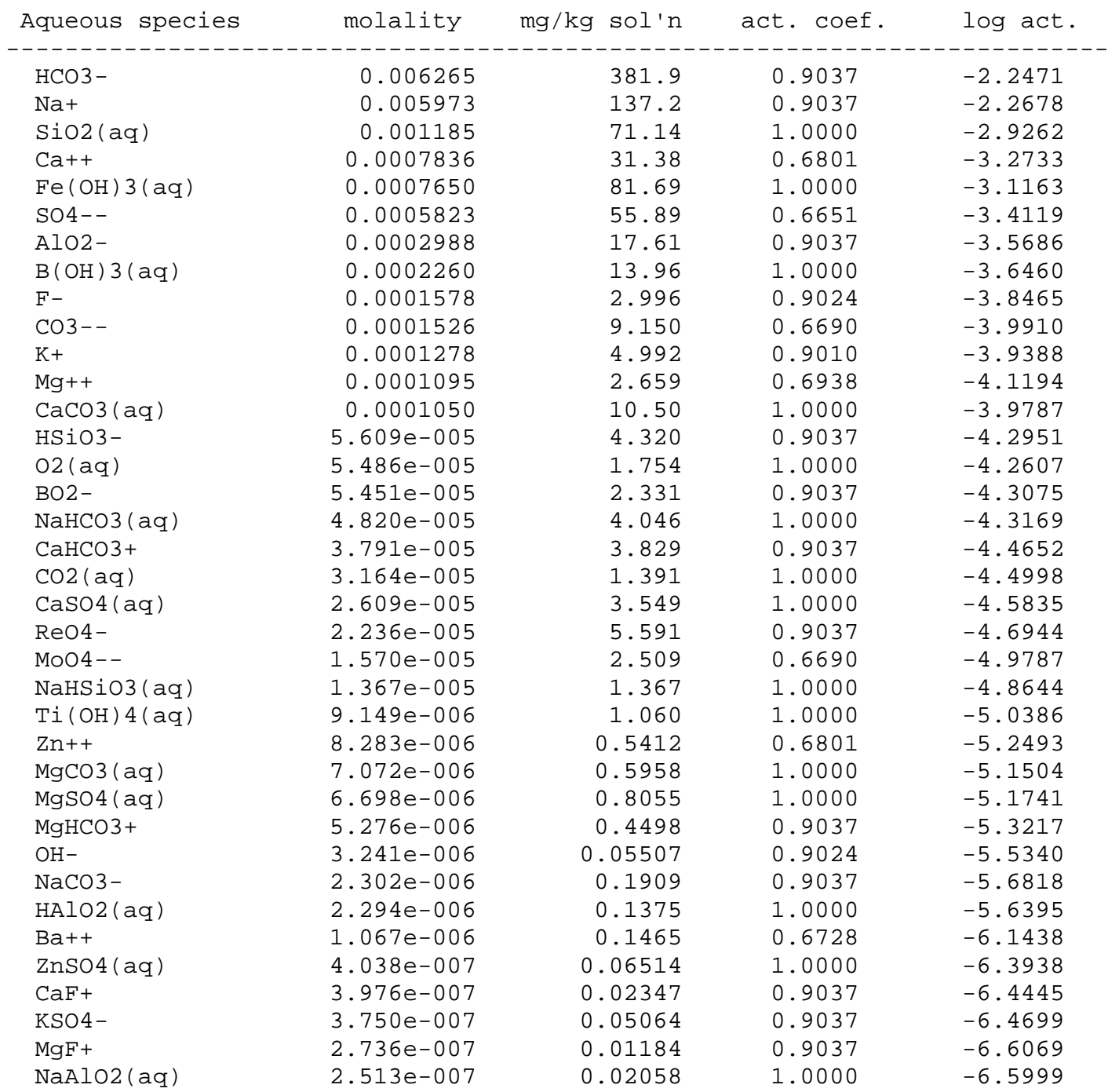




$\begin{array}{lcccc}\operatorname{NaF}(\mathrm{aq}) & 7.448 \mathrm{e}-0 \odot 8 & 0.0 \odot 3125 & 1.00 \odot \odot & -7.1279 \\ \mathrm{BaC03}(\mathrm{aq}) & 2.924 \mathrm{e}-0 \odot 8 & 0.005765 & 1.00 \odot \odot & -7.5340 \\ (\text { only species }>\text { 1e-8 molal listed) } & & \end{array}$

Mineral saturation states $\log \mathrm{Q} / \mathrm{K}$

$\log \mathrm{Q} / \mathrm{K}$

\begin{tabular}{|c|c|c|c|}
\hline Antigorite & $45.8993 \mathrm{~s} / \mathrm{sat}$ & Sanidine_high & $6.2192 \mathrm{~s} / \mathrm{sat}$ \\
\hline Clinoptilolite-h & $27.9193 \mathrm{~s} / \mathrm{sat}$ & Wairakite & $6.0895 \mathrm{~s} / \mathrm{sat}$ \\
\hline Clinoptilolite-C & $27.8903 \mathrm{~s} / \mathrm{sat}$ & Albite_low & $5.9979 \mathrm{~s} / \mathrm{sat}$ \\
\hline Clinoptilolite-h & $26.0645 \mathrm{~s} / \mathrm{sat}$ & Albite & $5.9978 \mathrm{~s} / \mathrm{sat}$ \\
\hline Clinoptilolite-N & $26.0637 \mathrm{~s} / \mathrm{sat}$ & Smectite-high-Fe & $5.3054 \mathrm{~s} / \mathrm{sat}$ \\
\hline Nontronite-Ca & $24.6393 \mathrm{~s} / \mathrm{sat}$ & Cordierite_hydr & $5.0953 \mathrm{~s} / \mathrm{sat}$ \\
\hline Nontronite-Mg & $24.5285 \mathrm{~s} / \mathrm{sat}$ & Albite_high & $4.6456 \mathrm{~s} / \mathrm{sat}$ \\
\hline Nontronite-Na & $24.3952 \mathrm{~s} / \mathrm{sat}$ & Diaspore & $3.7336 \mathrm{~s} / \mathrm{sat}$ \\
\hline Clinoptilolite-K & $24.2828 s /$ sat & Corundum & $3.3682 \mathrm{~s} / \mathrm{sat}$ \\
\hline Nontronite-K & $24.1976 \mathrm{~s} / \mathrm{sat}$ & Boehmite & $3.3211 \mathrm{~s} / \mathrm{sat}$ \\
\hline Clinoptilolite-h & $24.1730 \mathrm{~s} / \mathrm{sat}$ & Witherite & $3.3000 \mathrm{~s} / \mathrm{sat}$ \\
\hline Nontronite-H & $23.3094 \mathrm{~s} / \mathrm{sat}$ & Kyanite & $3.1890 \mathrm{~s} / \mathrm{sat}$ \\
\hline Stilbite & $18.5991 \mathrm{~s} / \mathrm{sat}$ & Anorthite & $3.1781 \mathrm{~s} / \mathrm{sat}$ \\
\hline Ferrite-Zn & $17.3857 \mathrm{~s} / \mathrm{sat}$ & Jadeite & $3.1684 \mathrm{~s} / \mathrm{sat}$ \\
\hline Hematite & $17.2758 \mathrm{~s} / \mathrm{sat}$ & Gibbsite & $3.1616 \mathrm{~s} / \mathrm{sat}$ \\
\hline Andradite & $16.8633 \mathrm{~s} / \mathrm{sat}$ & Andalusite & $2.9055 \mathrm{~s} / \mathrm{sat}$ \\
\hline Epidote-ord & $16.5741 \mathrm{~s} / \mathrm{sat}$ & Dolomite & $2.7076 \mathrm{~s} / \mathrm{sat}$ \\
\hline Epidote & $16.5737 \mathrm{~s} / \mathrm{sat}$ & Dolomite-ord & $2.7076 \mathrm{~s} / \mathrm{sat}$ \\
\hline Muscovite & $15.3834 \mathrm{~s} / \mathrm{sat}$ & Cordierite_anhyd & $2.5628 \mathrm{~s} / \mathrm{sat}$ \\
\hline Amesite-14A & $13.8913 \mathrm{~s} / \mathrm{sat}$ & Grossular & $2.5597 \mathrm{~s} / \mathrm{sat}$ \\
\hline Herschelite & $13.6636 \mathrm{~s} / \mathrm{sat}$ & Sillimanite & $2.5320 \mathrm{~s} / \mathrm{sat}$ \\
\hline Paragonite & $13.0195 \mathrm{~s} / \mathrm{sat}$ & Dawsonite & $2.1387 \mathrm{~s} / \mathrm{sat}$ \\
\hline Mesolite & $12.9793 \mathrm{~s} / \mathrm{sat}$ & Analcime-K & $2.0193 \mathrm{~s} / \mathrm{sat}$ \\
\hline Illite & $12.2619 \mathrm{~s} / \mathrm{sat}$ & Chrysotile & $1.9060 \mathrm{~s} / \mathrm{sat}$ \\
\hline Beidellite-Ca & $11.7693 \mathrm{~s} / \mathrm{sat}$ & Kalsilite & $1.8089 \mathrm{~s} / \mathrm{sat}$ \\
\hline Beidellite-Mg & $11.6583 \mathrm{~s} / \mathrm{sat}$ & Analcime & $1.6235 \mathrm{~s} / \mathrm{sat}$ \\
\hline Beidellite-Na & $11.5251 \mathrm{~s} / \mathrm{sat}$ & Anatase & $1.5200 \mathrm{~s} / \mathrm{sat}$ \\
\hline Beidellite-K & $11.3276 \mathrm{~s} / \mathrm{sat}$ & Quartz & $1.2056 \mathrm{~s} / \mathrm{sat}$ \\
\hline Scolecite & $11.2814 \mathrm{~s} / \mathrm{sat}$ & Calcite & $1.1973 \mathrm{~s} / \mathrm{sat}$ \\
\hline Montmor-Ca & $11.0315 \mathrm{~s} / \mathrm{sat}$ & Dolomite-dis & $1.1258 \mathrm{~s} / \mathrm{sat}$ \\
\hline Montmor-Mg & $10.9920 \mathrm{~s} / \mathrm{sat}$ & Aragonite & $1.0528 \mathrm{~s} / \mathrm{sat}$ \\
\hline Montmor-Na & $10.8552 \mathrm{~s} / \mathrm{sat}$ & Tridymite & 1.0079s/sat \\
\hline Montmor-K & $10.6617 \mathrm{~s} / \mathrm{sat}$ & Anthophyllite & $0.7102 \mathrm{~s} / \mathrm{sat}$ \\
\hline Laumontite & $10.6600 \mathrm{~s} / \mathrm{sat}$ & Cristobalite(alp & $0.6436 \mathrm{~s} / \mathrm{sat}$ \\
\hline Margarite & $10.4824 \mathrm{~s} / \mathrm{sat}$ & Chalcedony & $0.6297 \mathrm{~s} / \mathrm{sat}$ \\
\hline Clinochlore-14A & $10.4804 \mathrm{~s} / \mathrm{sat}$ & Smithsonite & $0.5929 \mathrm{~s} / \mathrm{sat}$ \\
\hline Beidellite-H & $10.4395 \mathrm{~s} / \mathrm{sat}$ & Zincite & $0.5523 \mathrm{~s} / \mathrm{sat}$ \\
\hline Pyrophyllite & $10.1329 \mathrm{~s} / \mathrm{sat}$ & Barite & $0.5108 \mathrm{~s} / \mathrm{sat}$ \\
\hline Celadonite & $9.8310 \mathrm{~s} / \mathrm{sat}$ & Nepheline & $0.4967 \mathrm{~s} / \mathrm{sat}$ \\
\hline Ferrite-Ca & $9.4570 \mathrm{~s} / \mathrm{sat}$ & Coesite & $0.3823 \mathrm{~s} / \mathrm{sat}$ \\
\hline Kaolinite & $9.3980 \mathrm{~s} / \mathrm{sat}$ & Monohydrocalcite & $0.3766 \mathrm{~s} / \mathrm{sat}$ \\
\hline Ferrite-Mg & $9.0332 \mathrm{~s} / \mathrm{sat}$ & Cronstedtite-7A & $0.2178 \mathrm{~s} / \mathrm{sat}$ \\
\hline Clinozoisite & $8.3705 \mathrm{~s} / \mathrm{sat}$ & Cristobalite(bet & $0.1855 \mathrm{~s} / \mathrm{sat}$ \\
\hline Phlogopite & $8.3493 \mathrm{~s} / \mathrm{sat}$ & $\mathrm{Zn}(\mathrm{OH}) 2($ gamma $)$ & $0.1475 \mathrm{~s} / \mathrm{sat}$ \\
\hline Zoisite & $8.3246 \mathrm{~s} / \mathrm{sat}$ & $\mathrm{Zn}(\mathrm{OH}) 2$ (epsilon) & $0.1222 \mathrm{~s} / \mathrm{sat}$ \\
\hline Tremolite & $8.1944 \mathrm{~s} / \mathrm{sat}$ & Diopside & $0.0081 \mathrm{~s} / \mathrm{sat}$ \\
\hline Goethite & $8.1634 \mathrm{~s} / \mathrm{sat}$ & Ice & -0.1194 \\
\hline Saponite-Ca & $7.9571 \mathrm{~s} / \mathrm{sat}$ & Si02(am) & -0.1225 \\
\hline Prehnite & $7.9110 \mathrm{~s} / \mathrm{sat}$ & Magnesite & -0.1497 \\
\hline Saponite-Mg & $7.8467 \mathrm{~s} / \mathrm{sat}$ & $\mathrm{Zn}(\mathrm{OH}) 2$ (beta) & -0.1537 \\
\hline Magnetite & $7.8162 \mathrm{~s} / \mathrm{sat}$ & $\mathrm{Fe}(\mathrm{OH}) 3$ & -0.5245 \\
\hline Lawsonite & $7.7191 \mathrm{~s} / \mathrm{sat}$ & Huntite & -0.8597 \\
\hline Saponite-Na & $7.7130 \mathrm{~s} / \mathrm{sat}$ & Fluorite & -0.8793 \\
\hline Saponite-K & $7.5154 \mathrm{~s} / \mathrm{sat}$ & Jarosite & -1.2232 \\
\hline Mordenite & $7.5131 \mathrm{~s} / \mathrm{sat}$ & Enstatite & -1.3114 \\
\hline Smectite-low-Fe- & $7.4860 \mathrm{~s} / \mathrm{sat}$ & Pargasite & -1.3171 \\
\hline
\end{tabular}




$\begin{array}{llll}\text { Maximum_Microcli } & 7.4523 \mathrm{~s} / \mathrm{sat} & \text { Analcime-dehy } & -1.8634 \\ \text { K-Feldspar } & 7.4512 \mathrm{~s} / \mathrm{sat} & \text { Gypsum } & -2.1948 \\ \text { Natrolite } & 7.4308 \mathrm{~s} / \mathrm{sat} & \text { Okenite } & -2.2279 \\ \text { Clinochlore-7A } & 7.0654 \mathrm{~s} / \mathrm{sat} & \text { Anhydrite } & -2.4197 \\ \text { Zn2Si04 } & 6.9202 \mathrm{~s} / \mathrm{sat} & \text { Sellaite } & -2.4524 \\ \text { Saponite-H } & 6.6272 \mathrm{~s} / \mathrm{sat} & \text { Wollastonite } & -2.8813 \\ \text { Talc } & 6.3177 \mathrm{~s} / \mathrm{sat} & & \\ \quad \text { (only minerals with } \operatorname{log~Q/K~}>-3 \text { listed) } & \end{array}$

\begin{tabular}{|c|c|c|}
\hline Gases & fugacity & $\log$ fug. \\
\hline & & \\
\hline $02(\mathrm{~g})$ & ○. . 03913 & -1.407 \\
\hline $\mathrm{H} 20$ (g) & ๑. 01903 & -1.721 \\
\hline $\operatorname{co2}(\mathrm{g})$ & $\odot .0 \odot \odot 7912$ & -3.102 \\
\hline$H F(g)$ & $1.649 e-014$ & -13.783 \\
\hline $\operatorname{SiF} 4(g)$ & $1.769 \mathrm{e}-038$ & -37.752 \\
\hline $\mathrm{BF} 3(\mathrm{~g})$ & $4.069 e-039$ & -38.390 \\
\hline H2(g) & $1.894 \mathrm{e}-042$ & -41.723 \\
\hline $\operatorname{co}(g)$ & $4.788 e-049$ & -48.320 \\
\hline So2(g) & $5.603 e-058$ & -57.252 \\
\hline $\mathrm{Zn}(\mathrm{g})$ & $1.145 \mathrm{e}-073$ & -72.941 \\
\hline $\mathrm{Na}(\mathrm{g})$ & $1.292 \mathrm{e}-075$ & -74.889 \\
\hline $\mathrm{K}(\mathrm{g})$ & $5.530 \mathrm{e}-078$ & -77.257 \\
\hline $\mathrm{F} 2(\mathrm{~g})$ & 1. $019 \mathrm{e}-084$ & -83.992 \\
\hline $\operatorname{Mg}(g)$ & $2.322 \mathrm{e}-130$ & -129.634 \\
\hline $\mathrm{H} 2 \mathrm{~S}(\mathrm{~g})$ & $7.542 e-146$ & -145.122 \\
\hline $\mathrm{CH} 4(\mathrm{~g})$ & $4.828 \mathrm{e}-147$ & -146.316 \\
\hline $\mathrm{Ca}(\mathrm{g})$ & $1.147 \mathrm{e}-152$ & -151.941 \\
\hline $\mathrm{Al}(\mathrm{g})$ & 8. $648 e-191$ & -190.063 \\
\hline$C(g)$ & 1. $912 \mathrm{e}-192$ & -191.718 \\
\hline$B(g)$ & $1.276 \mathrm{e}-205$ & -204.894 \\
\hline $\operatorname{Si}(g)$ & $2.841 e-223$ & -222.547 \\
\hline $\mathrm{s} 2(\mathrm{~g})$ & $1.074 e-233$ & -232.969 \\
\hline $\mathrm{C} 2 \mathrm{H} 4(\mathrm{~g})$ & $3.101 \mathrm{e}-240$ & -239.508 \\
\hline
\end{tabular}

\begin{tabular}{|c|c|c|c|c|c|}
\hline \multirow[b]{2}{*}{ Original basis } & \multirow[b]{2}{*}{ total moles } & \multicolumn{2}{|c|}{ In fluid } & Sorbed & \multirow{2}{*}{$\begin{array}{c}\mathrm{Kd} \\
\mathrm{L} / \mathrm{Kg}\end{array}$} \\
\hline & & moles & $\mathrm{mg} / \mathrm{kg}$ & moles $\quad \mathrm{mg} / \mathrm{kg}$ & \\
\hline & - - - - - - - - - & ------- & ------ & & \\
\hline $\mathrm{Al}+++$ & $\odot .000301$ & $\odot .000301$ & 8.12 & & \\
\hline $\mathrm{B}(\mathrm{OH}) 3(\mathrm{aq})$ & 0.000280 & 0.000280 & 17.3 & & \\
\hline $\mathrm{Ba}++$ & $1.10 \mathrm{e}-006$ & $1.10 \mathrm{e}-006$ & 0.150 & & \\
\hline $\mathrm{Ca}++$ & 0.000953 & 0.000953 & 38.2 & & \\
\hline $\mathrm{F}-$ & $\odot .000159$ & $\odot .000159$ & 3.01 & & \\
\hline $\mathrm{Fe}++$ & $\odot .00 \odot 765$ & $\odot .000765$ & 42.7 & & \\
\hline $\mathrm{H}+$ & -0.00310 & $-\odot .00310$ & -3.12 & & \\
\hline $\mathrm{H} 2 \mathrm{O}$ & 55.5 & 55.5 & $9.99 \mathrm{e}+\odot \odot 5$ & & \\
\hline $\mathrm{HCO}-$ & 0.00665 & 0.00665 & 406 & & \\
\hline $\mathrm{K}+$ & $\odot .000128$ & $\odot .000128$ & 5.01 & & \\
\hline $\mathrm{Mg}++$ & ๑. . 000129 & $\odot .000129$ & 3.13 & & \\
\hline Mo04 - - & $1.57 e-005$ & $1.57 \mathrm{e}-005$ & 2.51 & & \\
\hline $\mathrm{Na}+$ & 0.00604 & 0.00604 & 139. & & \\
\hline $02(\mathrm{aq})$ & $\odot .000246$ & $\odot .000246$ & 7.87 & & \\
\hline Re04 - & $2.24 e-005$ & $2.24 e-005$ & 5.59 & & \\
\hline SO4 - - & ๑. . 000616 & $\odot .000616$ & 59.1 & & \\
\hline $\operatorname{Si02}(\mathrm{aq})$ & 0.00125 & 0.00125 & 75.3 & & \\
\hline $\mathrm{Ti}(\mathrm{OH}) 4(\mathrm{aq})$ & $9.15 e-006$ & $9.15 e-006$ & 1.06 & & \\
\hline $\mathrm{Zn++}$ & $8.69 e-006$ & $8.69 e-006$ & 0.568 & & \\
\hline
\end{tabular}

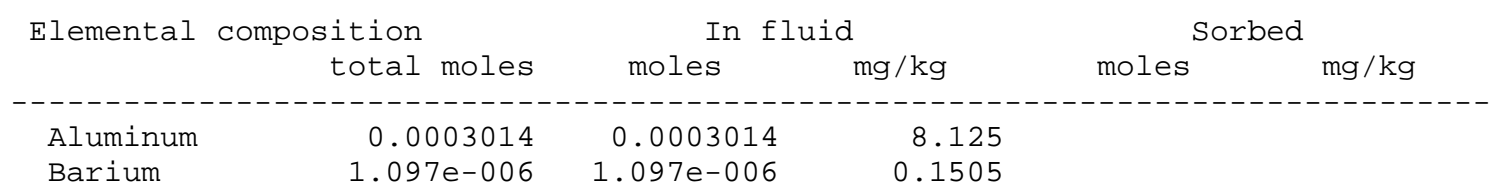




\begin{tabular}{|c|c|c|c|}
\hline Boron & 0.0002805 & $\odot .0002805$ & 3.029 \\
\hline Calcium & $\odot .0 \odot \odot 9531$ & ๑.๑०९9531 & 38.16 \\
\hline Carbon & ๑.๑๑6655 & 0.006655 & 79.86 \\
\hline Fluorine & $\odot .0001586$ & ๑. 0001586 & 3.010 \\
\hline Hydrogen & 111.0 & 111.0 & $1.118 \mathrm{e}+005$ \\
\hline Iron & 0.0007650 & 0.0007650 & 42.69 \\
\hline Magnesium & $\odot .0 \odot \odot 1288$ & 0.0001288 & 3.128 \\
\hline Molybdenum & $1.570 \mathrm{e}-005$ & $1.570 \mathrm{e}-005$ & 1.505 \\
\hline Oxygen & 55.54 & 55.54 & $8.878 \mathrm{e}+005$ \\
\hline Potassium & 0.0001282 & 0.00001282 & 5.007 \\
\hline Rhenium & $2.236 \mathrm{e}-005$ & $2.236 \mathrm{e}-005$ & 4.161 \\
\hline Silicon & 0.001255 & 0.001255 & 35.21 \\
\hline Sodium & ๑. $006 \odot 38$ & $\odot .0 \odot 6 \odot 38$ & 138.7 \\
\hline Sulfur & $\odot .0006159$ & $\odot .0006159$ & 19.73 \\
\hline Titanium & $9.149 \mathrm{e}-006$ & $9.149 \mathrm{e}-006$ & $\odot .4377$ \\
\hline Zinc & $8.687 \mathrm{e}-006$ & $8.687 \mathrm{e}-006$ & 0.5675 \\
\hline
\end{tabular}




\section{Sample D11BG}

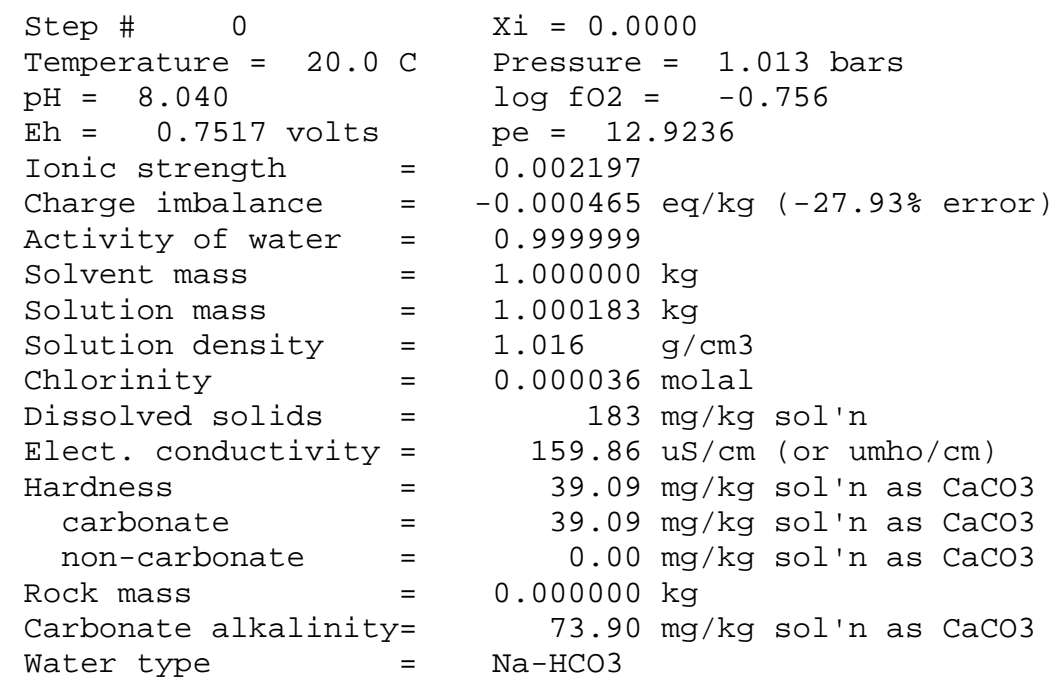

No minerals in system.

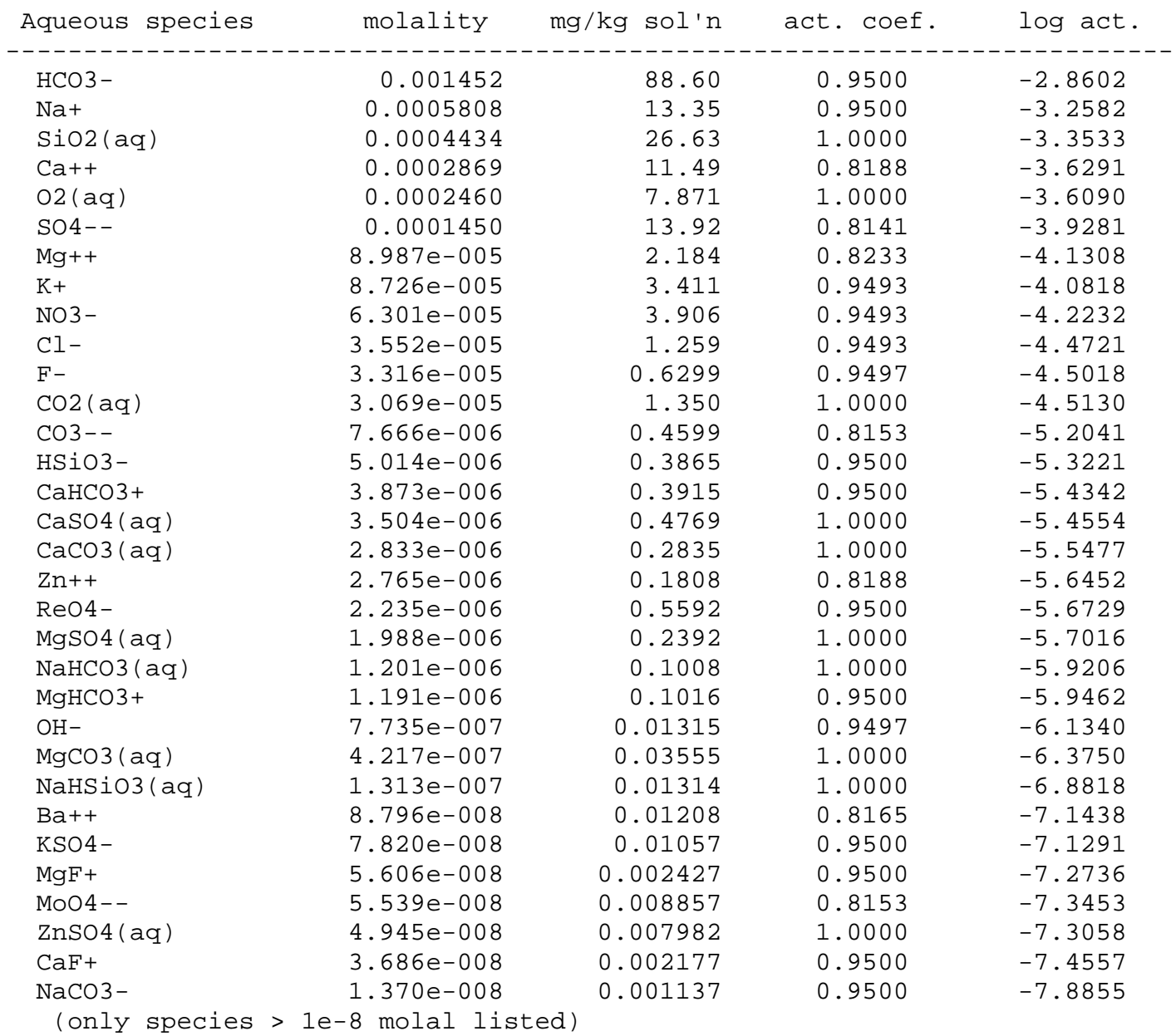




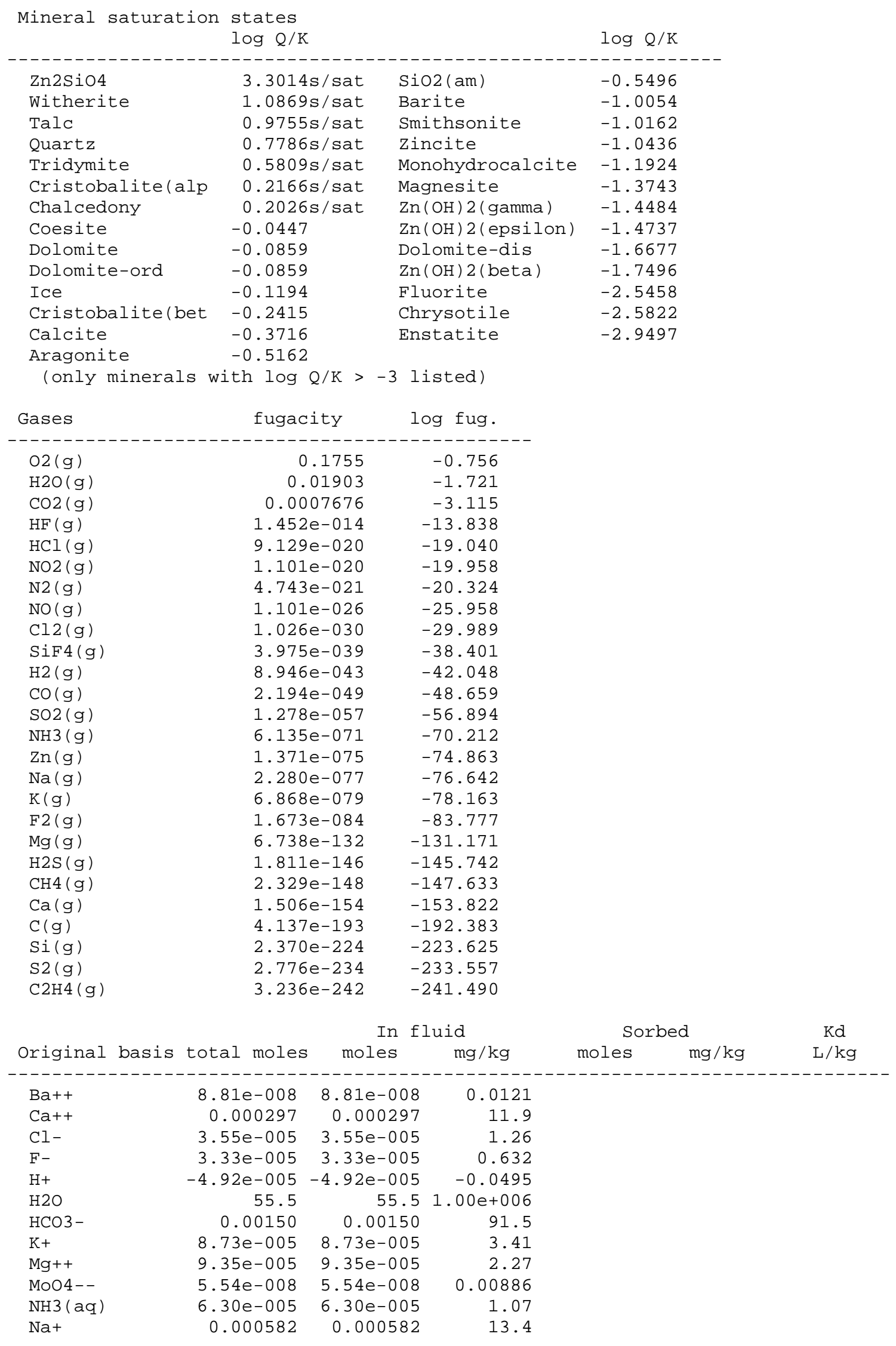




\begin{tabular}{|c|c|c|c|c|}
\hline $02(\mathrm{aq})$ & $\odot .0 \odot \odot 372$ & 0.000372 & 11.9 & \\
\hline ReO4- & $2.24 \mathrm{e}-006$ & $2.24 \mathrm{e}-\odot \odot 6$ & $\odot .559$ & \\
\hline SO4 - - & $\odot .0 \odot \odot 151$ & $\odot .0 \odot \odot 151$ & 14.5 & \\
\hline $\operatorname{Si02}(\mathrm{aq})$ & 0.000448 & 0.000448 & 26.9 & \\
\hline $\mathrm{Zn++}$ & $2.81 e-0 \odot 6$ & $2.81 \mathrm{e}-006$ & $\odot .184$ & \\
\hline \multicolumn{4}{|c|}{ Elemental composition } & Sorbed \\
\hline & total moles & moles & $\mathrm{mg} / \mathrm{kg}$ & moles $\quad \mathrm{mg} / \mathrm{kg}$ \\
\hline Barium & $8.814 \mathrm{e}-\odot \odot 8$ & $8.814 \mathrm{e}-\odot \odot 8$ & 0.01210 & \\
\hline Calcium & $\odot .0002971$ & $\odot .0002971$ & 11.91 & \\
\hline Carbon & $\odot .00150 \odot$ & $\odot .00150 \odot$ & 18.01 & \\
\hline Chlorine & $3.553 e-005$ & $3.553 e-0 \odot 5$ & 1.259 & \\
\hline Fluorine & $3.325 e-\odot \odot 5$ & $3.325 e-\odot \odot 5$ & 0.6317 & \\
\hline Hydrogen & 111.0 & 111.0 & $1.119 \mathrm{e}+0 \odot 5$ & \\
\hline Magnesium & $9.353 e-\odot \odot 5$ & $9.353 e-\odot \odot 5$ & 2.273 & \\
\hline Molybdenum & $5.539 \mathrm{e}-\odot \odot 8$ & $5.539 \mathrm{e}-\odot \odot 8$ & 0.005313 & \\
\hline Nitrogen & $6.301 \mathrm{e}-\odot \odot 5$ & $6.301 \mathrm{e}-005$ & ๑. 8824 & \\
\hline oxygen & 55.52 & 55.52 & $8.880 \mathrm{e}+\odot \odot 5$ & \\
\hline Potassium & $8.734 \mathrm{e}-\odot \odot 5$ & $8.734 \mathrm{e}-\odot \odot 5$ & 3.414 & \\
\hline Rhenium & $2.235 \mathrm{e}-\odot \odot 6$ & $2.235 \mathrm{e}-\odot \odot 6$ & 0.4162 & \\
\hline Silicon & $\odot .00 \odot 4485$ & $\odot .00 \odot 4485$ & 12.59 & \\
\hline Sodium & 0.0005821 & 0.0005821 & 13.38 & \\
\hline Sulfur & $\odot .0001506$ & $\odot .0001506$ & 4.828 & \\
\hline Zinc & $2.814 \mathrm{e}-\odot \odot 6$ & $2.814 \mathrm{e}-\odot \odot 6$ & $\odot .1840$ & \\
\hline
\end{tabular}




\section{Sample D11WTOP}

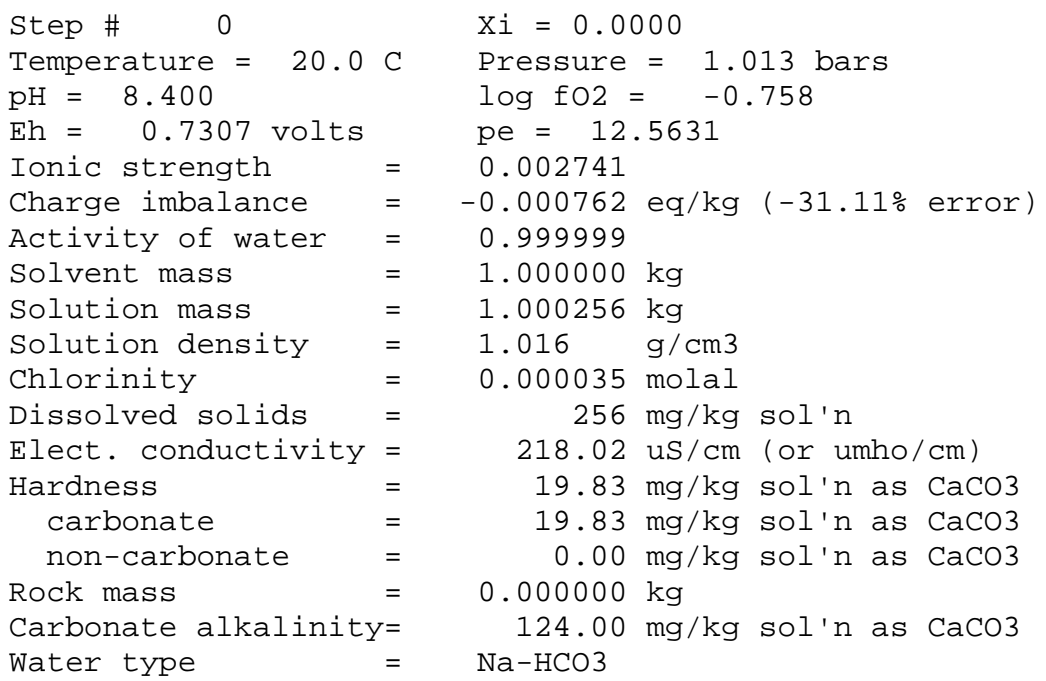

No minerals in system.

\begin{tabular}{|c|c|c|c|c|}
\hline Aqueous species & molality & $\mathrm{mg} / \mathrm{kg}$ sol'n & act. coef. & $\log$ act. \\
\hline 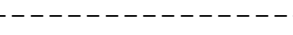 & 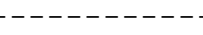 & 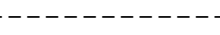 & - - - - - - - & - - - - - - \\
\hline HCO3 - & $\odot .0 \odot 24 \odot 3$ & 146.6 & $\odot .9447$ & -2.6439 \\
\hline $\mathrm{Na}+$ & $\odot .001624$ & 37.34 & $\odot .9447$ & -2.8140 \\
\hline $\operatorname{Si02}(\mathrm{aq})$ & $\odot .00 \odot 4397$ & 26.41 & 1.0000 & -3.3568 \\
\hline $02(\mathrm{aq})$ & 0.0002449 & 7.833 & 1.0000 & -3.6111 \\
\hline $\mathrm{Ca}++$ & ๑ . $0 \odot \odot 1644$ & 6.589 & 0.8017 & -3.8800 \\
\hline N03- & $8.682 e-005$ & 5.382 & 0.9439 & -4.0865 \\
\hline SO4- - & $7.505 e-005$ & 7.208 & 0.7960 & -4.2237 \\
\hline $\mathrm{F}-$ & $7.140 \mathrm{e}-005$ & 1.356 & 0.9443 & -4.1712 \\
\hline $\mathrm{K}+$ & $6.692 \mathrm{e}-005$ & 2.616 & 0.9439 & -4.1995 \\
\hline $\mathrm{Cl}-$ & $3.469 \mathrm{e}-0 \odot 5$ & 1.229 & $\odot .9439$ & -4.4849 \\
\hline C03-- & $2.954 \mathrm{e}-005$ & 1.773 & $\odot .7974$ & -4.6278 \\
\hline $\operatorname{co2}(\mathrm{aq})$ & $2.205 e-005$ & 0.9700 & 1.0000 & -4.6567 \\
\hline $\mathrm{Mg}++$ & $2.199 \mathrm{e}-\odot \odot 5$ & $\odot .5344$ & 0.8071 & -4.7508 \\
\hline HSiO3 - & $1.146 \mathrm{e}-005$ & 0.8830 & $\odot .9447$ & -4.9656 \\
\hline Al02- & $8.163 e-006$ & 0.4813 & 0.9447 & -5.1128 \\
\hline Cac03(aq) & $5.995 e-0 \odot 6$ & $\odot .5999$ & $1.00 \odot \odot$ & -5.2222 \\
\hline $\operatorname{NaHCO} 3(\mathrm{aq})$ & $5.496 e-0 \odot 6$ & 0.4616 & 1.0000 & -5.2600 \\
\hline $\mathrm{Fe}(\mathrm{OH}) 3(\mathrm{aq})$ & $4.687 e-0 \odot 6$ & $\odot .5008$ & 1..$\odot \odot \odot$ & -5.3291 \\
\hline $\mathrm{CaHCO} 3+$ & $3.597 \mathrm{e}-0 \odot 6$ & $\odot .3636$ & $\odot .9447$ & -5.4687 \\
\hline Re04 - & $2.965 \mathrm{e}-006$ & 0.7416 & 0.9447 & -5.5527 \\
\hline $\mathrm{OH}-$ & $1.782 \mathrm{e}-0 \odot 6$ & ๑. .03030 & $\odot .9443$ & -5.7740 \\
\hline CaSO4(aq) & $9.955 \mathrm{e}-0 \odot 7$ & 0.1355 & 1.0000 & -6.0019 \\
\hline NaHSiO3(aq) & $8.296 \mathrm{e}-\odot \odot 7$ & ๑. 08300 & $1.0 \odot \odot \odot$ & -6.0811 \\
\hline $\mathrm{MgHCO} 3+$ & $4.730 \mathrm{e}-\odot \odot 7$ & ๑. .04034 & $\odot .9447$ & -6.3499 \\
\hline Mo04 - - & $4.083 e-0 \odot 7$ & 0.06528 & 0.7974 & -6.4874 \\
\hline $\operatorname{MgC0} 3(\mathrm{aq})$ & $3.814 \mathrm{e}-0 \odot 7$ & ๑.03215 & $1.000 \odot$ & -6.4186 \\
\hline $\operatorname{MgSO} 4(\mathrm{aq})$ & $2.414 \mathrm{e}-0 \odot 7$ & ๑. 02905 & 1.0000 & -6.6172 \\
\hline $\mathrm{NaCO} 3-$ & 1. $445 \mathrm{e}-007$ & 0.01199 & 0.9447 & -6.8649 \\
\hline HAl02 (aq) & $1.138 \mathrm{e}-0 \odot 7$ & ๑. 006826 & $1.0 \odot \odot \odot$ & -6.9438 \\
\hline $\mathrm{Ba}++$ & $6.828 \mathrm{e}-0 \odot 8$ & 0.009374 & 0.7989 & -7.2632 \\
\hline CaF+ & $4.454 \mathrm{e}-\odot \odot 8$ & ๑. 002631 & 0.9447 & -7.3759 \\
\hline KSO4 - & $3.036 e-0 \odot 8$ & ๑. .००4102 & 0.9447 & -7.5425 \\
\hline $\mathrm{MgF}+$ & $2.896 e-0 \odot 8$ & 0.001254 & $\odot .9447$ & -7.5629 \\
\hline $\operatorname{NaF}(\mathrm{aq})$ & 1. $.03 e-\odot \odot 8$ & $\odot .0 \odot \odot 42 \odot 9$ & 1.0000 & -7.9989 \\
\hline
\end{tabular}




\begin{tabular}{|c|c|c|c|}
\hline & $\log Q / K$ & & $\log Q / K$ \\
\hline & & & \\
\hline Nontronite-Ma & 18.0240s/sat & Boenmite & 2.01095/sal \\
\hline Nontronite-Mg & $17.9090 \mathrm{~s} / \mathrm{sat}$ & Saponite-K & $2.0055 s / s a t$ \\
\hline Nontronite-Na & $17.6997 \mathrm{~s} / \mathrm{sat}$ & Smectite-low-Fe- & $1.9582 \mathrm{~s} / \mathrm{sat}$ \\
\hline Nontronite-K & $17.5963 \mathrm{~s} / \mathrm{sat}$ & Natrolite & $1.9582 \mathrm{~s} / \mathrm{sat}$ \\
\hline Nontronite-H & $16.8733 \mathrm{~s} / \mathrm{sat}$ & Phlogopite & $1.9186 \mathrm{~s} / \mathrm{sat}$ \\
\hline Clinoptilolite-h & $15.2408 \mathrm{~s} / \mathrm{sat}$ & Gibbsite & $1.8573 \mathrm{~s} / \mathrm{sat}$ \\
\hline Clinoptilolite-C & $15.2118 \mathrm{~s} / \mathrm{sat}$ & Prehnite & $1.8374 \mathrm{~s} / \mathrm{sat}$ \\
\hline Hematite & $12.8503 \mathrm{~s} / \mathrm{sat}$ & Witherite & $1.5437 \mathrm{~s} / \mathrm{sat}$ \\
\hline Clinoptilolite-h & $12.5440 \mathrm{~s} / \mathrm{sat}$ & Saponite-H & $1.2826 \mathrm{~s} / \mathrm{sat}$ \\
\hline Clinoptilolite-N & $12.5431 \mathrm{~s} / \mathrm{sat}$ & Albite_high & $1.2634 \mathrm{~s} / \mathrm{sat}$ \\
\hline Clinoptilolite-K & $11.7521 \mathrm{~s} / \mathrm{sat}$ & Talc & $1.2614 \mathrm{~s} / \mathrm{sat}$ \\
\hline Clinoptilolite-h & $11.6423 \mathrm{~s} / \mathrm{sat}$ & Clinochlore-14A & $1.0234 \mathrm{~s} / \mathrm{sat}$ \\
\hline Stilbite & $11.6021 \mathrm{~s} / \mathrm{sat}$ & Magnetite & $1.0155 \mathrm{~s} / \mathrm{sat}$ \\
\hline Muscovite & $9.6782 \mathrm{~s} / \mathrm{sat}$ & Clinozoisite & $0.9927 \mathrm{~s} / \mathrm{sat}$ \\
\hline Epidote-ord & 8.2878 s/sat & Zoisite & $0.9468 \mathrm{~s} / \mathrm{sat}$ \\
\hline Epidote & $8.2874 \mathrm{~s} / \mathrm{sat}$ & Quartz & $0.7750 \mathrm{~s} / \mathrm{sat}$ \\
\hline Andradite & $7.8861 \mathrm{~s} / \mathrm{sat}$ & Corundum & $0.7596 \mathrm{~s} / \mathrm{sat}$ \\
\hline Mesolite & $7.8424 \mathrm{~s} / \mathrm{sat}$ & Wairakite & $0.6721 \mathrm{~s} / \mathrm{sat}$ \\
\hline Herschelite & 7.4697s/sat & Tridymite & $0.5774 \mathrm{~s} / \mathrm{sat}$ \\
\hline Illite & $7.1768 \mathrm{~s} / \mathrm{sat}$ & Jadeite & $0.2168 \mathrm{~s} / \mathrm{sat}$ \\
\hline Paragonite & $7.0288 \mathrm{~s} / \mathrm{sat}$ & Cristobalite(alp & $0.2130 \mathrm{~s} / \mathrm{sat}$ \\
\hline Beidellite-Ca & $6.9709 \mathrm{~s} / \mathrm{sat}$ & Chalcedony & $0.1991 \mathrm{~s} / \mathrm{sat}$ \\
\hline Beidellite-Mg & $6.8559 \mathrm{~s} / \mathrm{sat}$ & Dolomite & $0.1960 \mathrm{~s} / \mathrm{sat}$ \\
\hline Beidellite-Na & $6.6465 \mathrm{~s} / \mathrm{sat}$ & Dolomite-ord & $0.1960 \mathrm{~s} / \mathrm{sat}$ \\
\hline Montmor-Ca & $6.5851 \mathrm{~s} / \mathrm{sat}$ & Kyanite & $0.1499 \mathrm{~s} / \mathrm{sat}$ \\
\hline Beidellite-K & $6.5433 \mathrm{~s} / \mathrm{sat}$ & Calcite & -0.0461 \\
\hline Montmor-Mg & $6.5415 \mathrm{~s} / \mathrm{sat}$ & Coesite & -0.0482 \\
\hline Montmor-Na & $6.3286 \mathrm{~s} / \mathrm{sat}$ & Dawsonite & -0.1086 \\
\hline Scolecite & $6.2945 \mathrm{~s} / \mathrm{sat}$ & Ice & -0.1194 \\
\hline Montmor - K & $6.2293 \mathrm{~s} / \mathrm{sat}$ & Andalusite & -0.1336 \\
\hline Goethite & $5.9506 \mathrm{~s} / \mathrm{sat}$ & Aragonite & -0.1907 \\
\hline Kaolinite & $5.9284 \mathrm{~s} / \mathrm{sat}$ & Cristobalite(bet & -0.2450 \\
\hline Beidellite-H & $5.8204 \mathrm{~s} / \mathrm{sat}$ & Kalsilite & -0.4266 \\
\hline Pyrophyllite & $5.8021 \mathrm{~s} / \mathrm{sat}$ & Sillimanite & -0.5071 \\
\hline Laumontite & $5.2425 \mathrm{~s} / \mathrm{sat}$ & Si02(am) & -0.5531 \\
\hline Celadonite & $5.1924 \mathrm{~s} / \mathrm{sat}$ & Analcime-K & -0.5918 \\
\hline Maximum_Microcli & $4.3556 \mathrm{~s} / \mathrm{sat}$ & Monohydrocalcite & -0.8669 \\
\hline K-Feldspar & $4.3545 \mathrm{~s} / \mathrm{sat}$ & Smectite-high-Fe & -0.9460 \\
\hline Ferrite-Ca & $3.9448 \mathrm{~s} / \mathrm{sat}$ & Analcime & -1.2617 \\
\hline Mordenite & $3.5100 \mathrm{~s} / \mathrm{sat}$ & Anorthite & -1.3782 \\
\hline Ferrite-Mg & 3. 4963s/sat & Dolomite-dis & -1.3858 \\
\hline Amesite-14A & $3.3677 \mathrm{~s} / \mathrm{sat}$ & Magnesite & -1.4179 \\
\hline Margarite & $3.3176 \mathrm{~s} / \mathrm{sat}$ & Barite & -1.4205 \\
\hline Lawsonite & $3.1628 \mathrm{~s} / \mathrm{sat}$ & Nepheline & -2.0244 \\
\hline Sanidine_high & $3.1226 \mathrm{~s} / \mathrm{sat}$ & Fluorite & -2.1354 \\
\hline Albite_low & $2.6157 \mathrm{~s} / \mathrm{sat}$ & Chrysotile & -2.2892 \\
\hline Albite & $2.6156 \mathrm{~s} / \mathrm{sat}$ & Clinochlore-7A & -2.3915 \\
\hline Saponite-Ca & $2.4332 \mathrm{~s} / \mathrm{sat}$ & $\mathrm{Fe}(\mathrm{OH}) 3$ & -2.7372 \\
\hline Diaspore & $2.4293 \mathrm{~s} / \mathrm{sat}$ & Enstatite & -2.8533 \\
\hline Saponite-Mg & $2.3187 \mathrm{~s} / \mathrm{sat}$ & Tremolite & -2.9802 \\
\hline Saponite-Na & $2.1089 \mathrm{~s} / \mathrm{sat}$ & & \\
\hline (only minerals & th $\log Q / K>$ & listed) & \\
\hline Gases & fugacity & $\log$ fug. & \\
\hline-------1 & $\ldots-\cdots$ & ------- & \\
\hline $02(g)$ & 0.1747 & -0.758 & \\
\hline $\mathrm{H} 20$ (g) & ๑. 01903 & -1.721 & \\
\hline $\mathrm{CO2}(\mathrm{g})$ & $\odot .00 \odot 5514$ & -3.259 & \\
\hline $\mathrm{HF}(\mathrm{g})$ & $1.357 \mathrm{e}-014$ & -13.867 & \\
\hline $\mathrm{HCl}(\mathrm{g})$ & $3.869 e-020$ & $-19 \cdot 412$ & \\
\hline
\end{tabular}




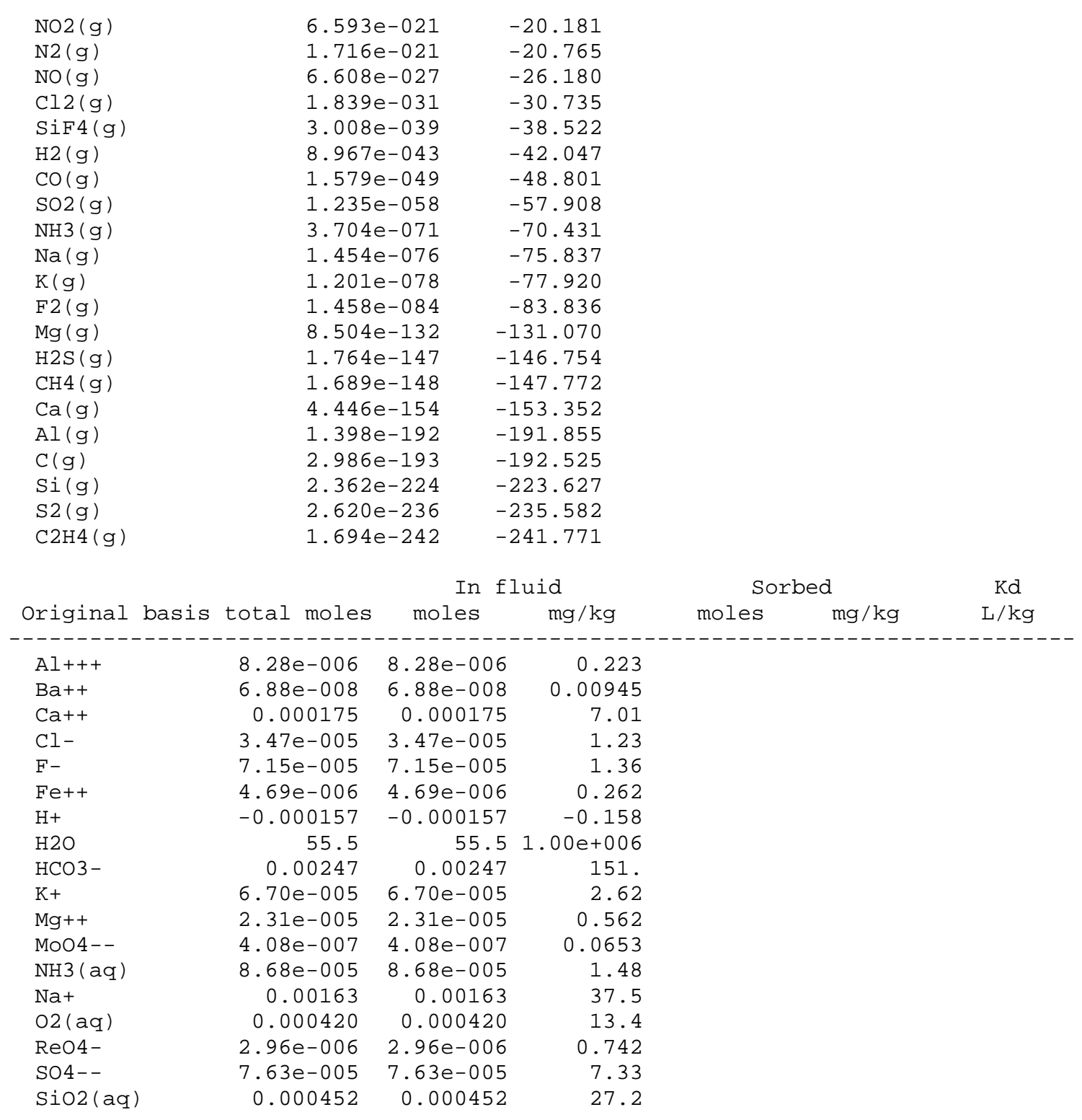




\begin{tabular}{|c|c|c|c|c|c|}
\hline \multicolumn{2}{|c|}{ Elemental composition } & \multicolumn{2}{|c|}{ In fluid } & \multicolumn{2}{|c|}{ Sorbed } \\
\hline & total moles & moles & $\mathrm{mg} / \mathrm{kg}$ & moles & $\mathrm{mg} / \mathrm{kg}$ \\
\hline & & & & & \\
\hline Aluminum & $8.280 \mathrm{e}-\odot \odot 6$ & $8.280 \mathrm{e}-006$ & 0.2233 & & \\
\hline Barium & $6.880 e-008$ & $6.880 e-008$ & $\odot .009445$ & & \\
\hline Calcium & $\odot .0001751$ & $\odot .0001751$ & 7.015 & & \\
\hline Carbon & $\odot .0 \odot 2471$ & ๑. 002471 & 29.67 & & \\
\hline Chlorine & $3.470 \mathrm{e}-\odot \odot 5$ & $3.470 \mathrm{e}-0 \odot 5$ & 1.230 & & \\
\hline Fluorine & $7.148 e-005$ & $7.148 e-005$ & 1.358 & & \\
\hline Hydrogen & 111.0 & 111.0 & $1.119 \mathrm{e}+005$ & & \\
\hline Iron & $4.687 e-006$ & $4.687 e-006$ & 0.2617 & & \\
\hline Magnesium & $2.312 \mathrm{e}-005$ & $2.312 \mathrm{e}-005$ & 0.5618 & & \\
\hline Molybdenum & $4.083 e-007$ & $4.083 e-007$ & ๑. . 03916 & & \\
\hline Nitrogen & $8.682 \mathrm{e}-005$ & $8.682 \mathrm{e}-005$ & 1.216 & & \\
\hline oxygen & 55.52 & 55.52 & $8.880 e+005$ & & \\
\hline Potassium & $6.695 e-005$ & $6.695 e-005$ & 2.617 & & \\
\hline Rhenium & $2.965 e-006$ & $2.965 e-006$ & 0.5519 & & \\
\hline Silicon & $\odot .0 \odot \odot 452 \odot$ & ๑. .0००4520 & 12.69 & & \\
\hline Sodium & ๑. . 001631 & 0.001631 & 37.49 & & \\
\hline Sulfur & $7.632 \mathrm{e}-005$ & $7.632 \mathrm{e}-005$ & 2.447 & & \\
\hline
\end{tabular}




\section{Sample D11NETOP}

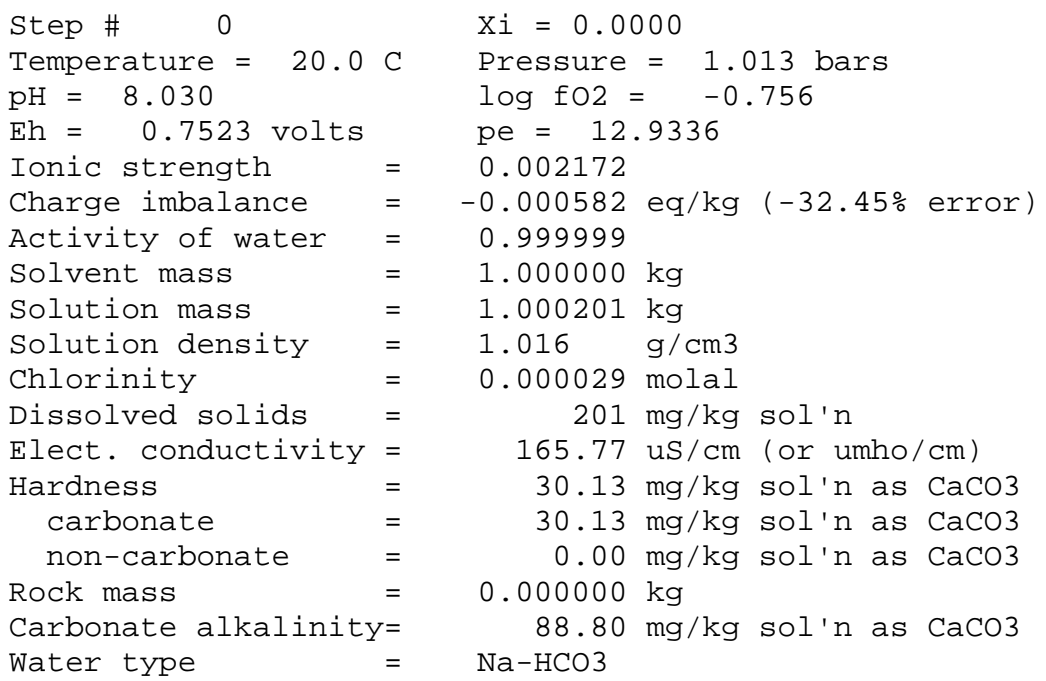

No minerals in system.

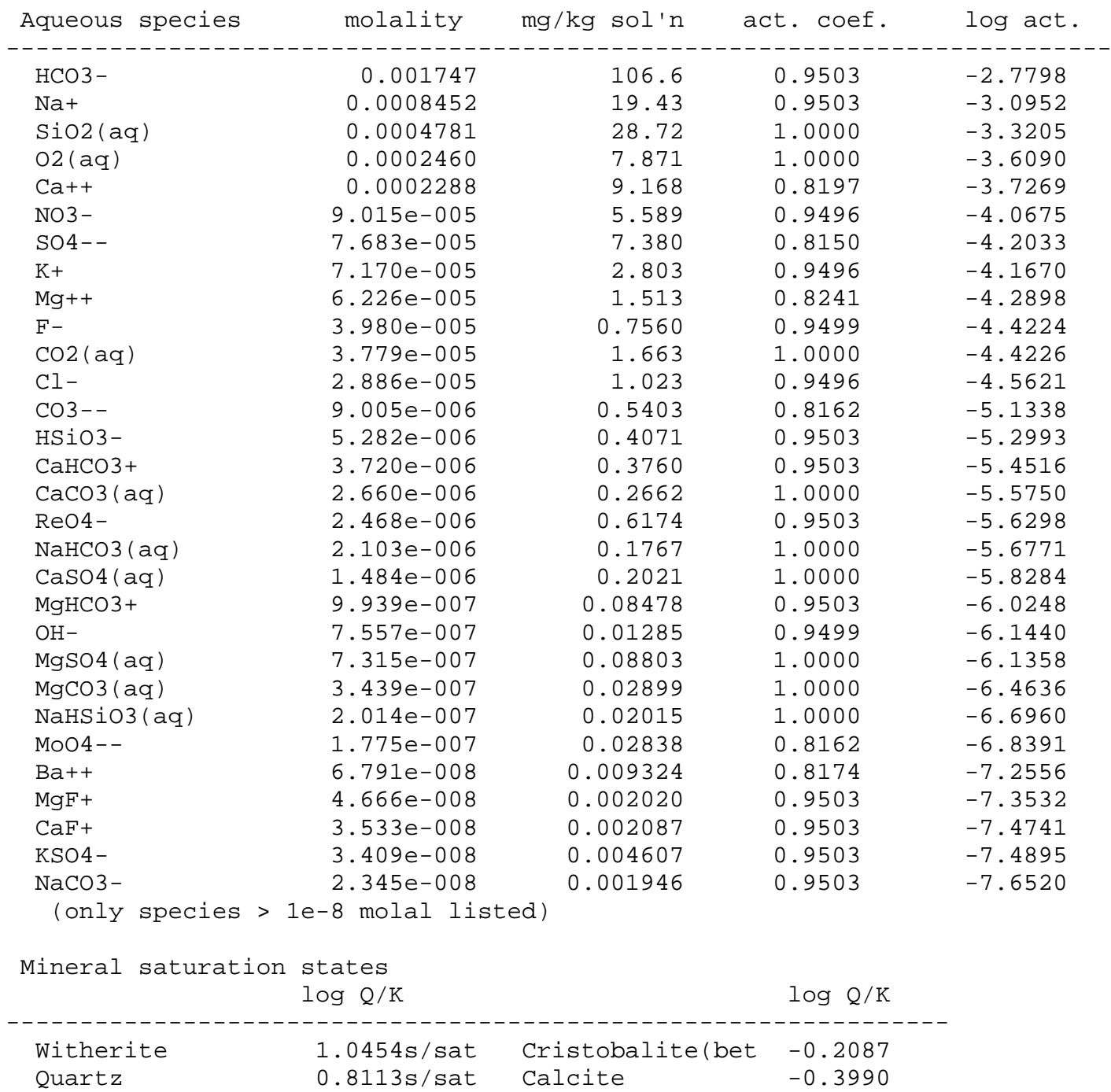




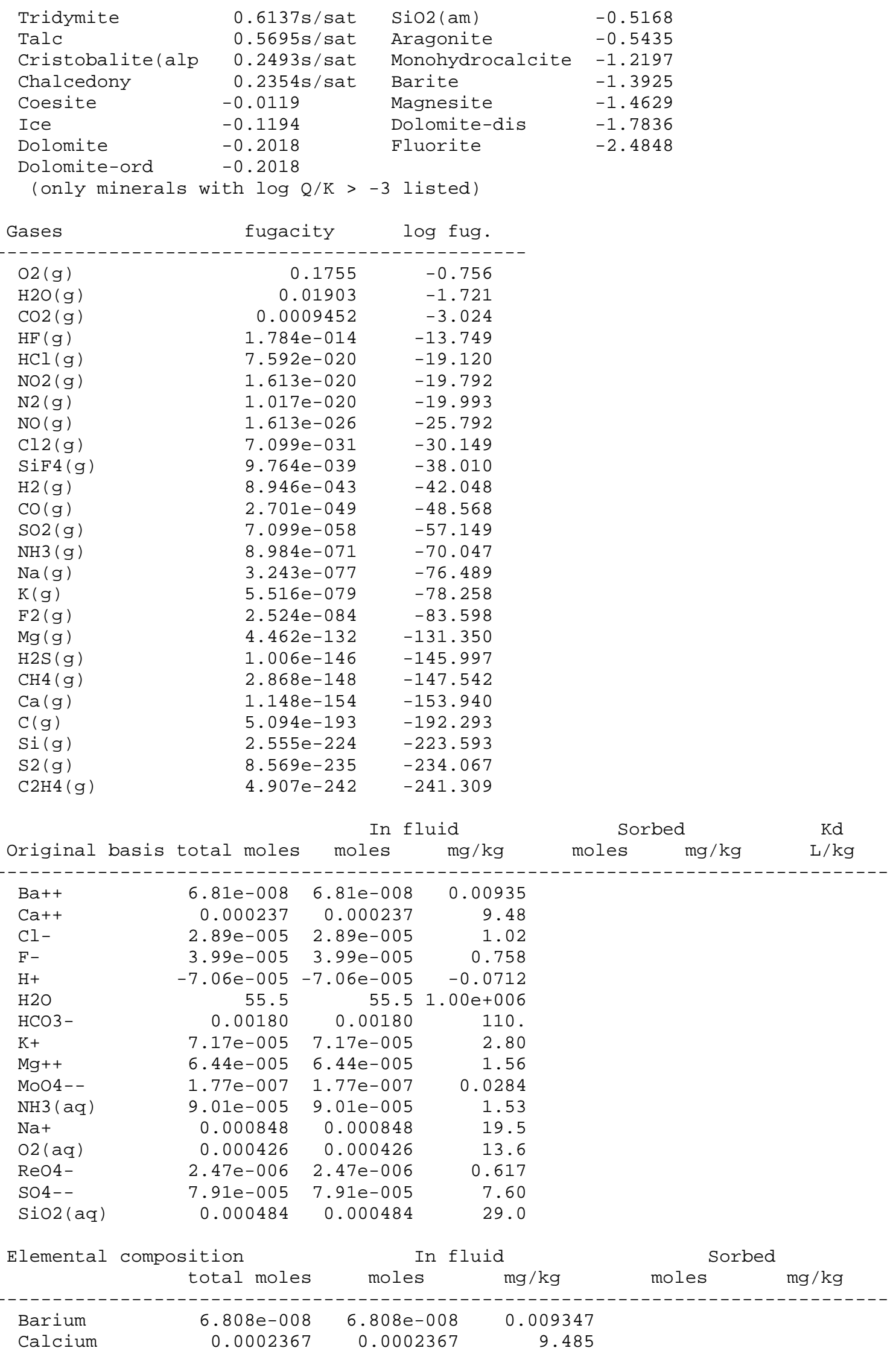




$\begin{array}{lrrr}\text { Carbon } & 0.001804 & 0.001804 & 21.66 \\ \text { Chlorine } & 2.887 \mathrm{e}-005 & 2.887 \mathrm{e}-005 & 1.023 \\ \text { Fluorine } & 3.988 \mathrm{e}-005 & 3.988 \mathrm{e}-005 & 0.7576 \\ \text { Hydrogen } & 111.0 & 111.0 & 1.119 \mathrm{e}+005 \\ \text { Magnesium } & 6.438 \mathrm{e}-005 & 6.438 \mathrm{e}-005 & 1.564 \\ \text { Molybdenum } & 1.775 \mathrm{e}-007 & 1.775 \mathrm{e}-007 & 0.01702 \\ \text { Nitrogen } & 9.015 \mathrm{e}-005 & 9.015 \mathrm{e}-005 & 1.262 \\ \text { Oxygen } & 55.52 & 55.52 & 8.880 \mathrm{e}+005 \\ \text { Potassium } & 7.173 \mathrm{e}-005 & 7.173 \mathrm{e}-005 & 2.804 \\ \text { Rhenium } & 2.468 \mathrm{e}-006 & 2.468 \mathrm{e}-006 & 0.4595 \\ \text { Silicon } & 0.0004835 & 0.00 \odot 4835 & 13.58 \\ \text { Sodium } & 0.0008475 & 0.0008475 & 19.48 \\ \text { Sulfur } & 7.908 \mathrm{e}-005 & 7.908 \mathrm{e}-005 & 2.535\end{array}$




\section{Sample D1119MMT_ts}

$\begin{array}{ll}\text { Step \# } \odot & \\ \text { Temperature }=20 . \odot \mathrm{C} \\ \text { pH }=9.010 \\ \text { Eh = } 0.6951 \text { volts } \\ \text { Ionic strength } \\ \text { Charge imbalance }= \\ \text { Activity of water }= \\ \text { Solvent mass } & = \\ \text { Solution mass } & = \\ \text { Solution density } & = \\ \text { Chlorinity } & = \\ \text { Dissolved solids } & = \\ \text { Elect. conductivity } & = \\ \text { Hardness } & = \\ \text { carbonate } & = \\ \text { non-carbonate } & = \\ \text { Rock mass } & = \\ \text { Carbonate alkalinity } & = \\ \text { Water type } & =\end{array}$

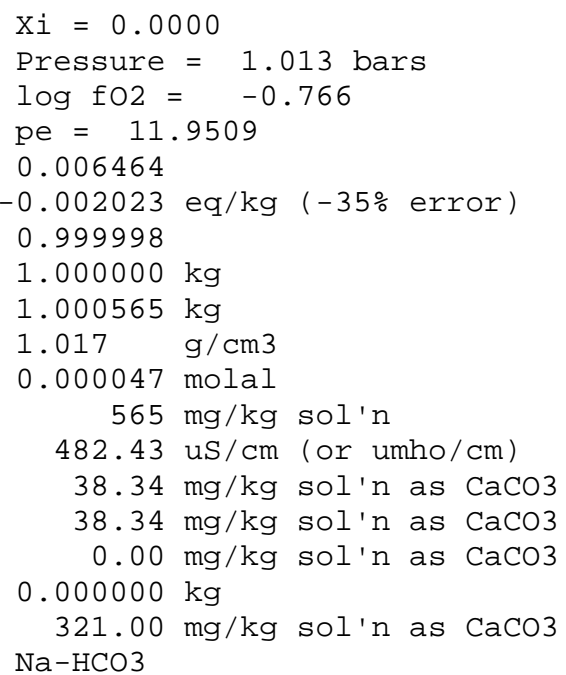

No minerals in system.

\begin{tabular}{|c|c|c|c|c|}
\hline Aqueous species & molality & $\mathrm{mg} / \mathrm{kg}$ sol'n & act. coef. & $\log$ act \\
\hline 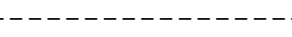 & ----- & $\ldots$ & - & \\
\hline HCO3 - & $\odot .005664$ & 345.4 & $\odot .9193$ & -2.2834 \\
\hline $\mathrm{Na}+$ & 0.004137 & 95.05 & $\odot .9193$ & -2.4199 \\
\hline Si02(aq) & $\odot .0006058$ & 36.38 & 1.0000 & -3.2177 \\
\hline CO3-- & ๑. . 0003076 & 18.45 & 0.7157 & -3.6573 \\
\hline $\mathrm{Ca}++$ & 0.0002636 & 10.56 & 0.7239 & -3.7193 \\
\hline $02(\mathrm{aq})$ & $\odot .0002400$ & 7.676 & 1.0000 & -3.6198 \\
\hline N03- & $9.572 \mathrm{e}-005$ & 5.932 & $\odot .9174$ & -4.0564 \\
\hline SO4 - - & $8.589 e-005$ & 8.247 & 0.7128 & -4.2130 \\
\hline CaC03(aq) & $8.110 \mathrm{e}-005$ & 8.112 & 1.0000 & -4.0910 \\
\hline $\mathrm{F}-$ & $8.069 e-005$ & 1.532 & $\odot .9183$ & -4.1302 \\
\hline HSi03 - & $6.608 e-005$ & 5.091 & ๑. 9193 & -4.2165 \\
\hline $\mathrm{K}+$ & $5.435 e-\odot \odot 5$ & 2.124 & $\odot .9174$ & -4.3022 \\
\hline $\mathrm{Cl}-$ & $4.717 \mathrm{e}-0 \odot 5$ & 1.671 & ๑. 9174 & -4.3638 \\
\hline Al02 - & 3. $961 \mathrm{e}-005$ & 2.335 & 0.9193 & -4.4388 \\
\hline $\mathrm{NaHCO} 3(\mathrm{aq})$ & $3.123 e-0 \odot 5$ & 2.622 & $1.00 \odot \odot$ & -4.5054 \\
\hline $\mathrm{Fe}(\mathrm{OH}) 3(\mathrm{aq})$ & $2.362 \mathrm{e}-005$ & 2.523 & 1.0000 & -4.6268 \\
\hline $\mathrm{Mg}++$ & $2.047 e-005$ & 0.4971 & 0.7342 & -4.8232 \\
\hline $\operatorname{co2}(\mathrm{aq})$ & $1.241 \mathrm{e}-005$ & 0.5459 & 1.0000 & -4.9062 \\
\hline CaHCO3+ & $1.227 \mathrm{e}-0 \odot 5$ & 1.240 & 0.9193 & -4.9475 \\
\hline NaHSiO3(aq) & $1.154 \mathrm{e}-\odot \odot 5$ & 1.154 & 1.0000 & -4.9379 \\
\hline $\mathrm{OH}-$ & $7.465 e-006$ & 0.1269 & 0.9183 & -5.1640 \\
\hline Re04 - & $4.223 e-006$ & 1.056 & $\odot .9193$ & -5.4109 \\
\hline NaC03- & $3.438 e-006$ & 0.2852 & $\odot .9193$ & -5.5003 \\
\hline $\operatorname{MgC03}(\mathrm{aq})$ & 3. $016 e-006$ & 0.2542 & 1.0000 & -5.5205 \\
\hline CaSO4 (aq) & $1.477 \mathrm{e}-\odot \odot 6$ & 0.2010 & $1 . \odot \odot \odot \odot$ & -5.8306 \\
\hline $\mathrm{MgHCO} 3+$ & $9.436 e-007$ & ๑.08047 & 0.9193 & -6.0618 \\
\hline Mo04 - - & $6.125 \mathrm{e}-0 \odot 7$ & ๑. 09790 & 0.7157 & -6.3582 \\
\hline Se04-- & $3.316 e-0 \odot 7$ & 0.04738 & 0.7128 & -6.6264 \\
\hline $\operatorname{MgS0} 4(\mathrm{aq})$ & $2.094 \mathrm{e}-007$ & ๑. 02520 & 1.0000 & -6.6789 \\
\hline $\mathrm{Ba}++$ & $1.847 e-007$ & 0.02535 & 0.7185 & -6.8770 \\
\hline $\mathrm{HAl02}(\mathrm{aq})$ & $1.319 e-007$ & $\odot .0 \odot 79 \odot 9$ & 1.0000 & -6.8797 \\
\hline $\mathrm{CaF}+$ & $7.284 \mathrm{e}-0 \odot 8$ & $\odot .0 \odot 4301$ & 0.9193 & -7.1742 \\
\hline $\mathrm{MgF}+$ & $2.769 e-008$ & ๑. .001198 & $\odot .9193$ & -7.5943 \\
\hline $\operatorname{NaF}(\mathrm{aq})$ & $2.730 \mathrm{e}-\odot \odot 8$ & $\odot .001146$ & $1.000 \odot$ & -7.5638 \\
\hline $\operatorname{NaCl}(a q)$ & $2.683 e-008$ & ๑. .0०1567 & 1.0000 & -7.5715 \\
\hline KSO4 - & $2.524 \mathrm{e}-0 \odot 8$ & 0.003409 & ๑.9193 & -7.6345 \\
\hline $\operatorname{NaAl02}(\mathrm{aq})$ & $2.386 e-0 \odot 8$ & ๑. 001955 & 1.0000 & -7.6223 \\
\hline
\end{tabular}




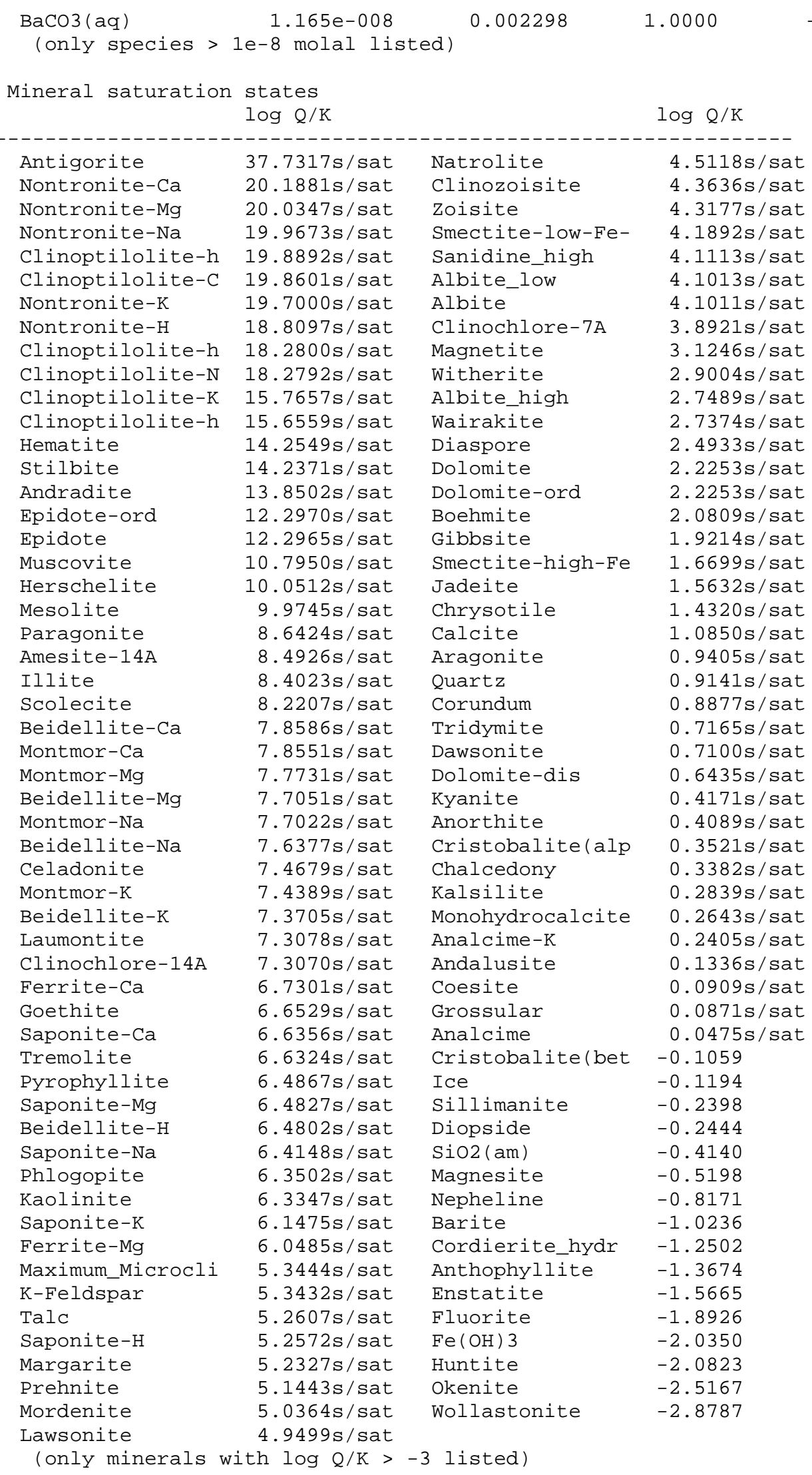




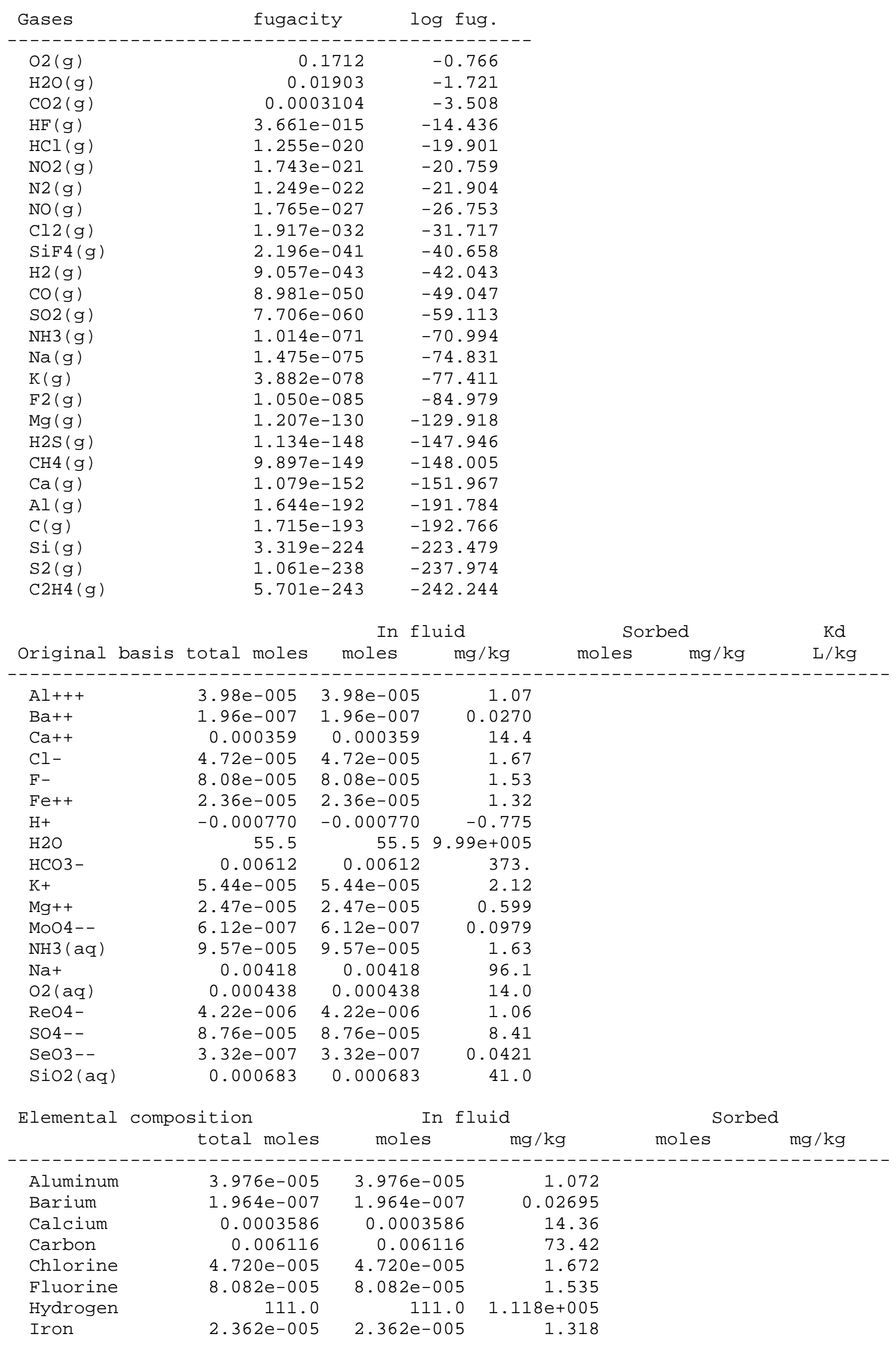




$\begin{array}{lrrr}\text { Magnesium } & 2.466 \mathrm{e}-\odot \odot 5 & 2.466 \mathrm{e}-005 & 0.5991 \\ \text { Molybdenum } & 6.125 \mathrm{e}-007 & 6.125 \mathrm{e}-007 & 0.05873 \\ \text { Nitrogen } & 9.572 \mathrm{e}-005 & 9.572 \mathrm{e}-005 & 1.340 \\ \text { Oxygen } & 55.53 & 55.53 & 8.879 \mathrm{e}+005 \\ \text { Potassium } & 5.438 \mathrm{e}-005 & 5.438 \mathrm{e}-005 & 2.125 \\ \text { Rhenium } & 4.223 \mathrm{e}-006 & 4.223 \mathrm{e}-006 & 0.7860 \\ \text { Selenium } & 3.316 \mathrm{e}-007 & 3.316 \mathrm{e}-007 & 0.02617 \\ \text { Silicon } & 0.0006834 & 0.0006834 & 19.18 \\ \text { Sodium } & 0.004183 & 0.004183 & 96.11 \\ \text { Sulfur } & 8.760 \mathrm{e}-005 & 8.760 \mathrm{e}-005 & 2.808\end{array}$




\section{Sample D1119MMT_bs}

$\begin{array}{ll}\text { Step \# } \odot & \\ \text { Temperature }=20 . \odot \mathrm{C} \\ \text { pH }=9.380 \\ \text { Eh }=0.6736 \text { volts } \\ \text { Ionic strength } & = \\ \text { Charge imbalance } & = \\ \text { Activity of water } & = \\ \text { Solvent mass } & = \\ \text { Solution mass } & = \\ \text { Solution density } & = \\ \text { Chlorinity } & = \\ \text { Dissolved solids } & = \\ \text { Elect. conductivity } & = \\ \text { Hardness } & = \\ \text { carbonate } & = \\ \text { non-carbonate } & = \\ \text { Rock mass } & = \\ \text { Carbonate alkalinity } & = \\ \text { Water type } & =\end{array}$

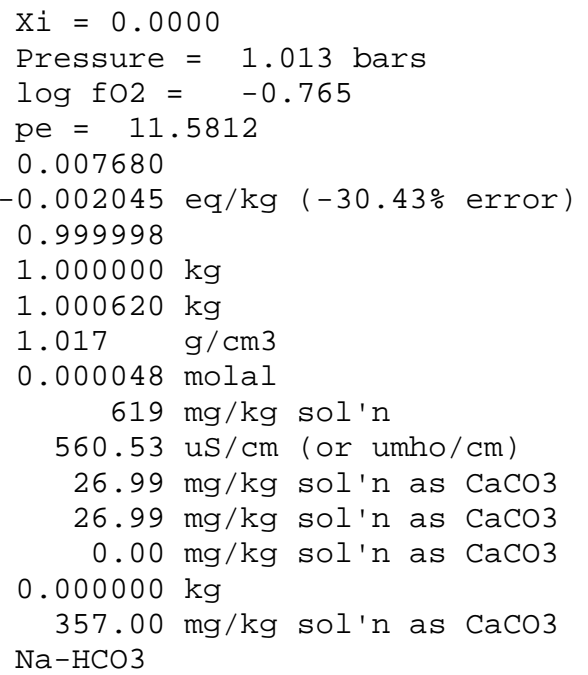

No minerals in system.

\begin{tabular}{|c|c|c|c|c|}
\hline Aqueous species & molality & $\mathrm{mg} / \mathrm{kg}$ sol'n & act. coef. & $\log$ act \\
\hline - - - - & ----- & $\ldots$ & - & \\
\hline $\mathrm{HCO} 3-$ & $\odot .005527$ & 337.0 & $\odot .9131$ & -2.2970 \\
\hline $\mathrm{Na}+$ & ๑. 005317 & 122.2 & $\odot .9131$ & -2.3138 \\
\hline CO3-- & $\odot .0007176$ & 43.04 & 0.6969 & -3.3009 \\
\hline $\operatorname{Si02}(\mathrm{aq})$ & 0.0005013 & 30.10 & 1.0000 & -3.2999 \\
\hline $02(\mathrm{aq})$ & 0.0002406 & 7.694 & 1.0000 & -3.6187 \\
\hline $\mathrm{F}-$ & $\odot .0 \odot \odot 1578$ & 2.997 & $\odot .9120$ & -3.8418 \\
\hline $\mathrm{Ca}++$ & $\odot .0001419$ & 5.684 & $\odot .7063$ & -3.9990 \\
\hline B02 - & 0.0001328 & 5.681 & 0.9131 & -3.9163 \\
\hline HSi03 - & ๑ . 0001290 & 9.942 & 0.9131 & -3.9288 \\
\hline $\mathrm{B}(\mathrm{OH}) 3(\mathrm{aq})$ & ๑. 0001012 & 6.254 & 1.0000 & -3.9948 \\
\hline $\operatorname{Cac03}(\mathrm{aq})$ & $9.675 e-005$ & 9.678 & 1.0000 & -4.0143 \\
\hline NO3- & $9.223 e-\odot \odot 5$ & 5.715 & $\odot .9109$ & -4.0756 \\
\hline SO4 - - & $6.496 e-005$ & 6.236 & 0.6937 & -4.3462 \\
\hline $\mathrm{K}+$ & $5.889 e-005$ & 2.301 & 0.9109 & -4.2705 \\
\hline $\mathrm{Cl}-$ & $4.800 \mathrm{e}-0 \odot 5$ & 1.701 & $\odot .9109$ & -4.3593 \\
\hline $\operatorname{NaHCO} 3(\mathrm{aq})$ & $3.865 e-005$ & 3.244 & 1.0000 & -4.4129 \\
\hline Al02- & $3.458 e-005$ & 2.038 & 0.9131 & -4.5006 \\
\hline NaHSiO3(aq) & $2.857 e-005$ & 2.858 & 1.0000 & -4.5441 \\
\hline $\mathrm{Fe}(\mathrm{OH}) 3(\mathrm{aq})$ & $2.045 e-005$ & 2.184 & 1.0000 & -4.6894 \\
\hline $\mathrm{OH}-$ & $1.762 \mathrm{e}-005$ & $\odot .2995$ & $\odot .9120$ & -4.7940 \\
\hline $\mathrm{Mg}++$ & $1.750 \mathrm{e}-005$ & 0.4251 & 0.7179 & -4.9009 \\
\hline Re04 - & $1.126 e-005$ & 2.815 & 0.9131 & -4.9880 \\
\hline NaC03 - & $1.004 \mathrm{e}-005$ & 0.8327 & ๑. 9131 & -5.0378 \\
\hline HPO4 - - & $9.315 e-0 \odot 6$ & 0.8935 & 0.6937 & -5.1897 \\
\hline CaHCO3+ & $6.289 e-0 \odot 6$ & 0.6354 & $\odot .9131$ & -5.2409 \\
\hline $\operatorname{MgC03}(\mathrm{aq})$ & $5.730 \mathrm{e}-006$ & 0.4829 & 1.0000 & -5.2418 \\
\hline $\mathrm{co} 2(\mathrm{aq})$ & $5.131 \mathrm{e}-006$ & 0.2257 & 1.0000 & -5.2898 \\
\hline Mo04 - - & $1.826 e-006$ & ๑. 2919 & $\odot .6969$ & -5.8953 \\
\hline $\mathrm{MgHCO}+$ & $7.699 \mathrm{e}-007$ & ๑.06565 & $\odot .9131$ & -6.1531 \\
\hline Se04-- & $7.417 e-007$ & 0.1060 & 0.6937 & -6.2886 \\
\hline CaSo4(aq) & $5.709 \mathrm{e}-\odot \odot 7$ & 0.07767 & 1.0000 & -6.2435 \\
\hline $\mathrm{Ba}++$ & $1.327 \mathrm{e}-0 \odot 7$ & 0.01821 & 0.7001 & -7.0320 \\
\hline $\operatorname{MgS0} 4(\mathrm{aq})$ & $1.289 \mathrm{e}-007$ & ๑. 01550 & 1.0000 & -6.8898 \\
\hline $\mathrm{CaF}+$ & $7.481 \mathrm{e}-0 \odot 8$ & $\odot .004417$ & 0.9131 & -7.1655 \\
\hline $\operatorname{NaF}(\mathrm{aq})$ & $6.772 \mathrm{e}-008$ & $\odot .002842$ & 1.0000 & -7.1693 \\
\hline $\mathrm{H} 2 \mathrm{PO} 4-$ & $4.972 \mathrm{e}-008$ & ๑. 004819 & ๑. 9131 & -7.3430 \\
\hline HAl02 (aq) & $4.880 \mathrm{e}-\odot \odot 8$ & $\odot .0 \odot 2926$ & 1.0000 & -7.3116 \\
\hline
\end{tabular}




\begin{tabular}{|c|c|c|c|c|}
\hline $\mathrm{MgF}+$ & $4.528 e-0 \odot 8$ & ๑.001959 & 0.9131 & -7.3836 \\
\hline $\operatorname{NaCl}(\mathrm{aq})$ & $3.461 \mathrm{e}-008$ & $\odot .002021$ & 1.0000 & -7.4609 \\
\hline $\mathrm{NaAlO2}(\mathrm{aq})$ & $2.643 e-0 \odot 8$ & 0.002165 & 1.0000 & -7.5780 \\
\hline KSO4 - & $2.012 \mathrm{e}-008$ & 0.002717 & 0.9131 & -7.7359 \\
\hline $\operatorname{BaC03}(\mathrm{aq})$ & $1.853 \mathrm{e}-\odot \odot 8$ & $\odot .003654$ & 1.0000 & -7.7322 \\
\hline P04 - - - & 1. $490 \mathrm{e}-\odot \odot 8$ & ๑. . 001415 & 0.4387 & -8.1845 \\
\hline $\mathrm{NaOH}(\mathrm{aq})$ & 1. $288 \mathrm{e}-\odot \odot 8$ & $\odot .0 \odot \odot 5147$ & $1.000 \odot$ & -7.8902 \\
\hline
\end{tabular}

Mineral saturation states $\log \mathrm{Q} / \mathrm{K}$

$\log \mathrm{Q} / \mathrm{K}$

\begin{tabular}{|c|c|c|c|}
\hline Antigorite & $66.7259 \mathrm{~s} / \mathrm{sat}$ & Natrolite & $4.3536 \mathrm{~s} / \mathrm{s}$ \\
\hline Nontronite-Ca & $19.6944 \mathrm{~s} / \mathrm{sat}$ & Smectite-low-Fe- & $4.0698 \mathrm{~s} / \mathrm{s}$ \\
\hline Nontronite-Mg & $19.5744 \mathrm{~s} / \mathrm{sat}$ & Albite_low & $3.8988 \mathrm{~s} / \mathrm{s}$ \\
\hline Nontronite-Na & $19.5548 \mathrm{~s} / \mathrm{sat}$ & Albite & $3.8987 \mathrm{~s} / \mathrm{s}$ \\
\hline Nontronite-K & $19.2630 \mathrm{~s} / \mathrm{sat}$ & Sanidine_high & $3.8344 \mathrm{~s}$ \\
\hline Nontronite-H & $18.2401 \mathrm{~s} / \mathrm{sat}$ & Margarite & $3.8011 \mathrm{~s}$ \\
\hline Clinoptilolite-h & $18.0008 \mathrm{~s} / \mathrm{sat}$ & Clinozoisite & $3.7419 \mathrm{~s} /$ \\
\hline Clinoptilolite-C & $17.9718 \mathrm{~s} / \mathrm{sat}$ & Zoisite & $3.6959 \mathrm{~s} /$ \\
\hline Clinoptilolite-h & $17.2445 \mathrm{~s} / \mathrm{sat}$ & Chrysotile & $3.2544 \mathrm{~s} / \mathrm{s}$ \\
\hline Clinoptilolite-N & $17.2437 \mathrm{~s} / \mathrm{sat}$ & Witherite & $3.1019 \mathrm{~s} / \mathrm{s}$ \\
\hline Andradite & $14.8590 \mathrm{~s} / \mathrm{sat}$ & Magnetite & $2.9364 \mathrm{~s} /$ \\
\hline Clinoptilolite-K & $14.4724 \mathrm{~s} / \mathrm{sat}$ & Anthophyllite & $2.6107 \mathrm{~s}$ \\
\hline Clinoptilolite-h & $14.3626 \mathrm{~s} / \mathrm{sat}$ & Dolomite & $2.5806 \mathrm{~s} / \mathrm{s}$ \\
\hline Hematite & 14.1297s/sat & Dolomite-ord & $2.5806 \mathrm{~s} / \mathrm{s}$ \\
\hline Fluorapatite & $13.5000 \mathrm{~s} / \mathrm{sat}$ & Albite_high & $2.5465 \mathrm{~s} /$ \\
\hline Stilbite & $13.2710 \mathrm{~s} / \mathrm{sat}$ & Diaspore & $2.0615 \mathrm{~s} /$ \\
\hline Epidote-ord & $12.0445 \mathrm{~s} / \mathrm{sat}$ & Wairakite & $2.0050 \mathrm{~s} /$ \\
\hline Epidote & $12.0440 \mathrm{~s} / \mathrm{sat}$ & Smectite-high-Fe & $1.6993 \mathrm{~s} /$ \\
\hline Tremolite & $10.2064 \mathrm{~s} / \mathrm{sat}$ & Boehmite & $1.6491 \mathrm{~s} /$ \\
\hline Herschelite & $9.7184 \mathrm{~s} / \mathrm{sat}$ & Gibbsite & $1.4895 \mathrm{~s} /$ \\
\hline Muscovite & $9.6544 \mathrm{~s} / \mathrm{sat}$ & Jadeite & $1.4430 \mathrm{~s} /$ \\
\hline Clinochlore-14A & $9.5081 \mathrm{~s} / \mathrm{sat}$ & Calcite & $1.1617 \mathrm{~s} /$ \\
\hline Mesolite & $9.4918 \mathrm{~s} / \mathrm{sat}$ & Aragonite & $1.0171 \mathrm{~s} /$ \\
\hline Amesite-14A & $9.2499 \mathrm{~s} / \mathrm{sat}$ & Dolomite-dis & $0.9988 \mathrm{~s}$ \\
\hline Saponite-Ca & 8.2541s/sat & Quartz & $0.8319 \mathrm{~s} /$ \\
\hline Saponite-Mg & $8.1345 \mathrm{~s} / \mathrm{sat}$ & Diopside & $0.7137 \mathrm{~s} /$ \\
\hline Saponite-Na & $8.1145 \mathrm{~s} / \mathrm{sat}$ & Tridymite & $0.6343 \mathrm{~s} /$ \\
\hline Phlogopite & $8.0603 \mathrm{~s} / \mathrm{sat}$ & Dawsonite & $0.3706 \mathrm{~s} /$ \\
\hline Saponite-K & $7.8226 \mathrm{~s} / \mathrm{sat}$ & Grossular & $0.3575 \mathrm{~s}$ \\
\hline Celadonite & $7.7711 \mathrm{~s} / \mathrm{sat}$ & Monohydrocalcite & $0.3410 \mathrm{~s}$ \\
\hline Paragonite & $7.5763 \mathrm{~s} / \mathrm{sat}$ & Whitlockite & $0.2815 \mathrm{~s}$ \\
\hline Scolecite & $7.5705 \mathrm{~s} / \mathrm{sat}$ & Cristobalite(alp & $0.2699 \mathrm{~s} /$ \\
\hline Illite & $7.5279 \mathrm{~s} / \mathrm{sat}$ & Chalcedony & $0.2560 \mathrm{~s} /$ \\
\hline Montmor-Ca & 7.0994s/sat & Kalsilite & $0.1715 \mathrm{~s} /$ \\
\hline Ferrite-Ca & 7.0651s/sat & Analcime-K & $0.0438 \mathrm{~s} /$ \\
\hline Montmor-Mg & $7.0507 \mathrm{~s} / \mathrm{sat}$ & Corundum & $0.0240 \mathrm{~s} /$ \\
\hline Montmor-Na & $7.0277 \mathrm{~s} / \mathrm{sat}$ & Coesite & $0.0087 \mathrm{~s} /$ \\
\hline Talc & $6.9187 \mathrm{~s} / \mathrm{sat}$ & Analcime & $-\odot .0777$ \\
\hline Saponite-H & $6.7998 \mathrm{~s} / \mathrm{sat}$ & Ice & -0.1194 \\
\hline Montmor-K & $6.7399 \mathrm{~s} / \mathrm{sat}$ & Anorthite & -0.1591 \\
\hline Beidellite-Ca & $6.6265 \mathrm{~s} / \mathrm{sat}$ & Cristobalite(bet & -0.1882 \\
\hline Goethite & $6.5903 \mathrm{~s} / \mathrm{sat}$ & Magnesite & -0.2411 \\
\hline Ferrite-Mg & $6.5856 \mathrm{~s} / \mathrm{sat}$ & Si02(am) & -0.4962 \\
\hline Laumontite & $6.5754 \mathrm{~s} / \mathrm{sat}$ & Kyanite & $-\odot .5288$ \\
\hline Beidellite-Mg & $6.5063 \mathrm{~s} / \mathrm{sat}$ & Andalusite & -0.8123 \\
\hline Beidellite-Na & $6.4868 \mathrm{~s} / \mathrm{sat}$ & Nepheline & -0.8551 \\
\hline Beidellite-K & $6.1950 \mathrm{~s} / \mathrm{sat}$ & Enstatite & -0.9865 \\
\hline Clinochlore-7A & $6.0931 \mathrm{~s} / \mathrm{sat}$ & Huntite & -1.1695 \\
\hline Kaolinite & $5.3065 \mathrm{~s} / \mathrm{sat}$ & Sillimanite & -1.1858 \\
\hline Pyrophyllite & $5.2941 \mathrm{~s} / \mathrm{sat}$ & Barite & -1.3117 \\
\hline Beidellite-H & $5.1722 \mathrm{~s} / \mathrm{sat}$ & Fluorite & -1.5957 \\
\hline
\end{tabular}




$\begin{array}{llll}\text { Maximum_Microcli } & 5.0675 \mathrm{~s} / \mathrm{sat} & \text { Cordierite_hydr } & -2.0642 \\ \text { K-Feldspar } & 5.0664 \mathrm{~s} / \mathrm{sat} & \text { Fe }(\mathrm{OH}) 3 & -2.0976 \\ \text { Prehnite } & 4.9544 \mathrm{~s} / \mathrm{sat} & \text { Okenite } & -2.2209 \\ \text { Mordenite } & 4.5194 \mathrm{~s} / \mathrm{sat} & \text { Wollastonite } & -2.5007 \\ \text { Hydroxylapatite } & 4.4868 \mathrm{~s} / \mathrm{sat} & \text { Pseudowollastoni } & -2.7488 \\ \text { Lawsonite } & 4.3820 \mathrm{~s} / \mathrm{sat} & \text { Brucite } & -2.7778 \\ \quad \text { (only minerals with log } \mathrm{Q} / \mathrm{K}> & -3 \text { listed) } & \end{array}$

\begin{tabular}{|c|c|c|}
\hline Gases & fugacity & $\log$ fug. \\
\hline$---\cdot--$ & ------- & - - - - - - - \\
\hline $02(g)$ & $\odot .1716$ & -0.765 \\
\hline $\mathrm{H} 2 \mathrm{O}(\mathrm{g})$ & ๑. 01903 & -1.721 \\
\hline $\mathrm{CO} 2(\mathrm{~g})$ & ๑ . 0001283 & -3.892 \\
\hline $\mathrm{HF}(\mathrm{g})$ & $3.034 e-015$ & -14.518 \\
\hline $\mathrm{HCl}(\mathrm{g})$ & $5.410 \mathrm{e}-021$ & -20.267 \\
\hline N02 (g) & $7.110 \mathrm{e}-022$ & -21.148 \\
\hline N2 (g) & $2.067 e-023$ & -22.685 \\
\hline NO $(g)$ & $7.189 \mathrm{e}-028$ & -27.143 \\
\hline Cl2(g) & $3.565 e-033$ & -32.448 \\
\hline BF3(g) & $1.134 \mathrm{e}-041$ & -40.945 \\
\hline SiF4(g) & $8.566 \mathrm{e}-042$ & -41.067 \\
\hline $\mathrm{H} 2(\mathrm{~g})$ & $9.046 e-\odot 43$ & -42.044 \\
\hline $\operatorname{co}(g)$ & $3.709 \mathrm{e}-050$ & -49.431 \\
\hline $\mathrm{SO2}(\mathrm{g})$ & $1.031 \mathrm{e}-060$ & -59.987 \\
\hline NH3 $(g)$ & $4.118 e-072$ & -71.385 \\
\hline $\mathrm{Na}(\mathrm{g})$ & $4.413 e-075$ & -74.355 \\
\hline K(g) & $9.784 \mathrm{e}-078$ & -77.009 \\
\hline $\mathrm{F} 2(\mathrm{~g})$ & $7.221 e-086$ & -85.141 \\
\hline $\operatorname{Mg}(g)$ & $5.538 e-130$ & -129.257 \\
\hline $\mathrm{CH} 4(\mathrm{~g})$ & $4.071 e-149$ & -148.390 \\
\hline $\mathrm{H} 2 \mathrm{~S}(\mathrm{~g})$ & $1.511 \mathrm{e}-149$ & -148.821 \\
\hline $\mathrm{Ca}(\mathrm{g})$ & $3.110 \mathrm{e}-152$ & -151.507 \\
\hline $\mathrm{Al}(\mathrm{g})$ & $6.072 e-193$ & -192.217 \\
\hline$C(g)$ & $7.073 e-194$ & -193.150 \\
\hline$B(g)$ & $1.885 e-206$ & -205.725 \\
\hline Si(g) & $2.740 \mathrm{e}-224$ & -223.562 \\
\hline $\mathrm{S} 2(\mathrm{~g})$ & $1.889 \mathrm{e}-240$ & -239.724 \\
\hline $\mathrm{C} 2 \mathrm{H} 4(\mathrm{~g})$ & $9.671 e-244$ & -243.015 \\
\hline
\end{tabular}

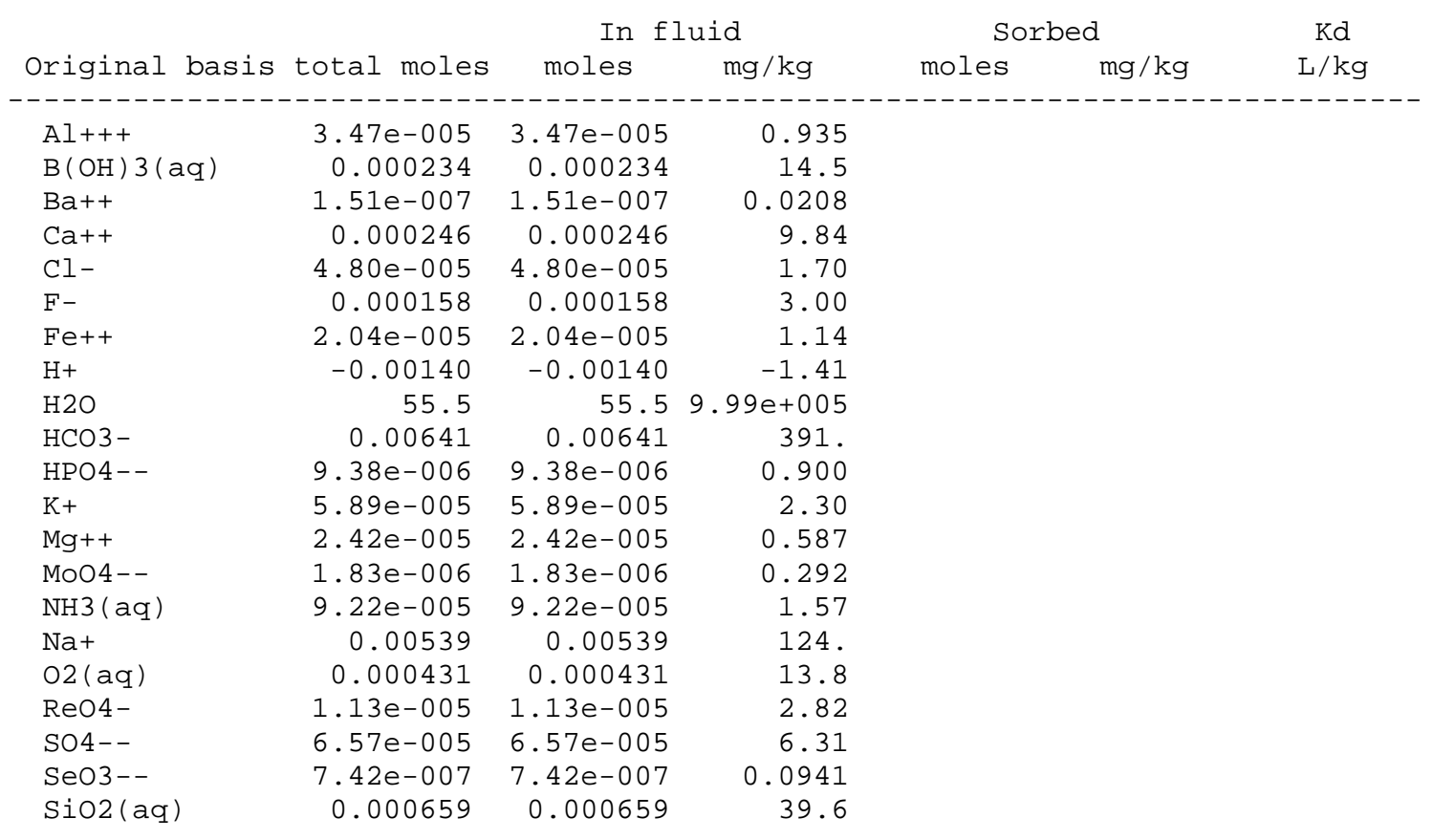




\begin{tabular}{|c|c|c|c|c|}
\hline \multirow{2}{*}{\multicolumn{2}{|c|}{$\begin{array}{l}\text { Elemental composition } \\
\text { total moles }\end{array}$}} & \multicolumn{2}{|c|}{ In fluid } & Sorbed \\
\hline & & moles & $\mathrm{mg} / \mathrm{kg}$ & moles $\quad \mathrm{mg} / \mathrm{kg}$ \\
\hline Aluminum & $3.466 \mathrm{e}-005$ & $3.466 \mathrm{e}-005$ & 0.9345 & \\
\hline Barium & $1.512 \mathrm{e}-\odot \odot 7$ & $1.512 \mathrm{e}-\odot \odot 7$ & $\odot .02076$ & \\
\hline Boron & $\odot .0 \odot \odot 234 \odot$ & $\odot .0 \odot \odot 2340$ & 2.528 & \\
\hline Calcium & 0.0002456 & $\odot .0002456$ & 9.837 & \\
\hline Carbon & $\odot .006408$ & $\odot .0 \odot 6408$ & 76.91 & \\
\hline Chlorine & $4.803 e-0 \odot 5$ & $4.803 e-005$ & 1.702 & \\
\hline Fluorine & $\odot .0 \odot \odot 1580$ & $\odot .0001580$ & 3.000 & \\
\hline Hydrogen & 111.0 & 111.0 & $1.118 \mathrm{e}+005$ & \\
\hline Iron & $2 . \odot 45 e-\odot \odot 5$ & $2.045 e-005$ & 1.141 & \\
\hline Magnesium & $2.418 \mathrm{e}-\odot \odot 5$ & $2.418 e-0 \odot 5$ & $\odot .5873$ & \\
\hline Molybdenum & $1.826 \mathrm{e}-0 \odot 6$ & $1.826 \mathrm{e}-0 \odot 6$ & 0.1751 & \\
\hline Nitrogen & $9.223 e-005$ & $9.223 e-005$ & 1.291 & \\
\hline oxygen & 55.53 & 55.53 & $8.879 \mathrm{e}+005$ & \\
\hline Phosphorus & $9.380 \mathrm{e}-\odot \odot 6$ & $9.380 \mathrm{e}-006$ & ๑. 2903 & \\
\hline Potassium & $5.891 e-005$ & $5.891 e-005$ & 2.302 & \\
\hline Rhenium & $1.126 \mathrm{e}-0 \odot 5$ & $1.126 \mathrm{e}-0 \odot 5$ & 2.095 & \\
\hline Selenium & $7.417 \mathrm{e}-0 \odot 7$ & $7.417 e-0 \odot 7$ & $\odot .05853$ & \\
\hline Silicon & $\odot .0 \odot \odot 6589$ & $\odot .0 \odot \odot 6589$ & 18.49 & \\
\hline Sodium & 0.005395 & 0.005395 & 123.9 & \\
\hline Sulfur & $6.568 \mathrm{e}-005$ & $6.568 e-0 \odot 5$ & 2.105 & \\
\hline
\end{tabular}




\section{Sample D11NBTW}

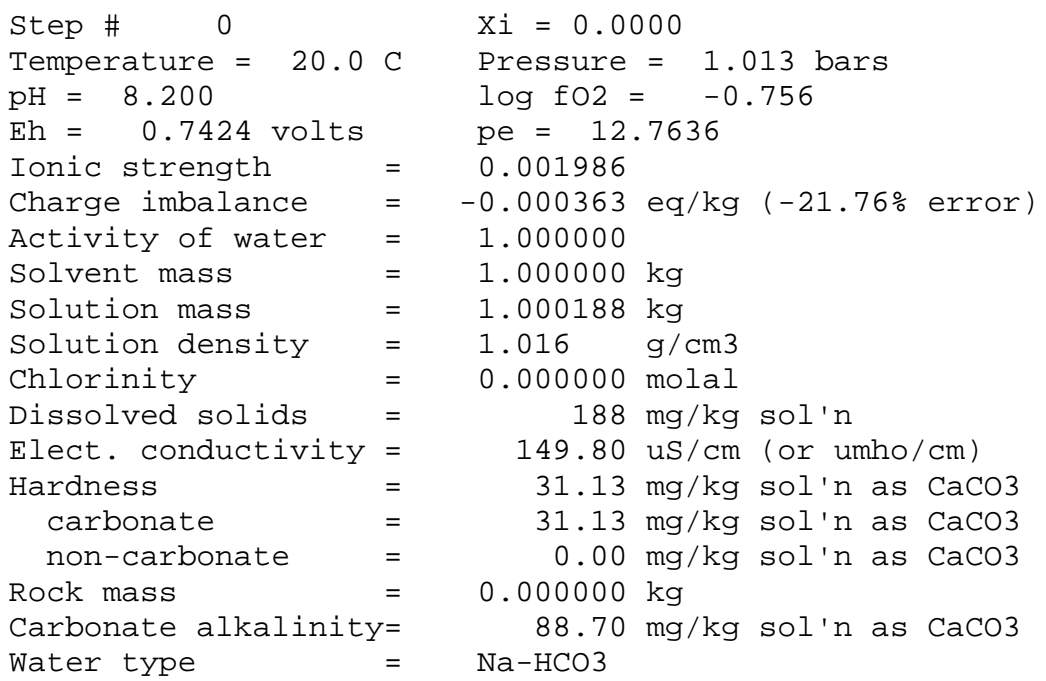

No minerals in system.

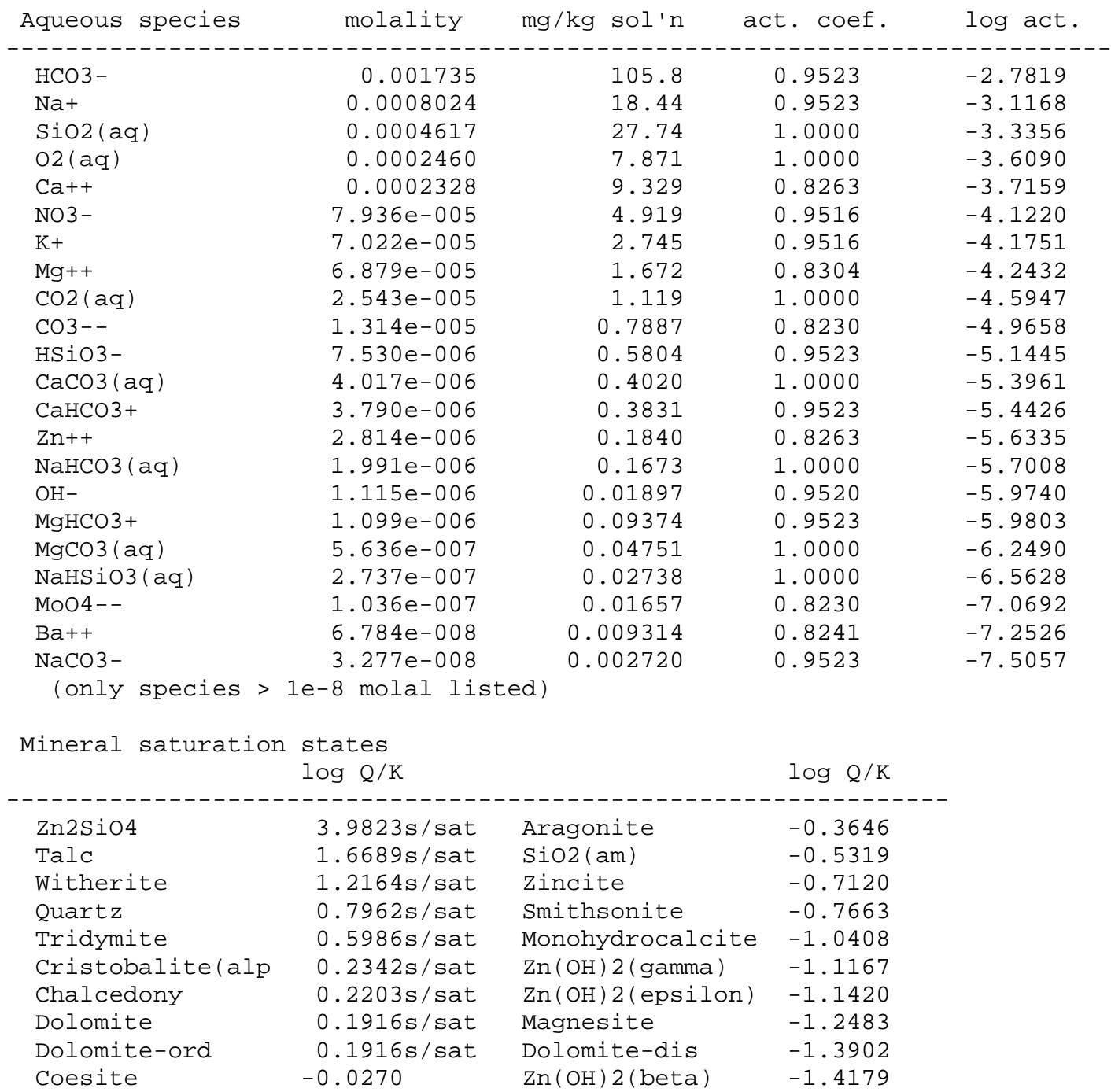




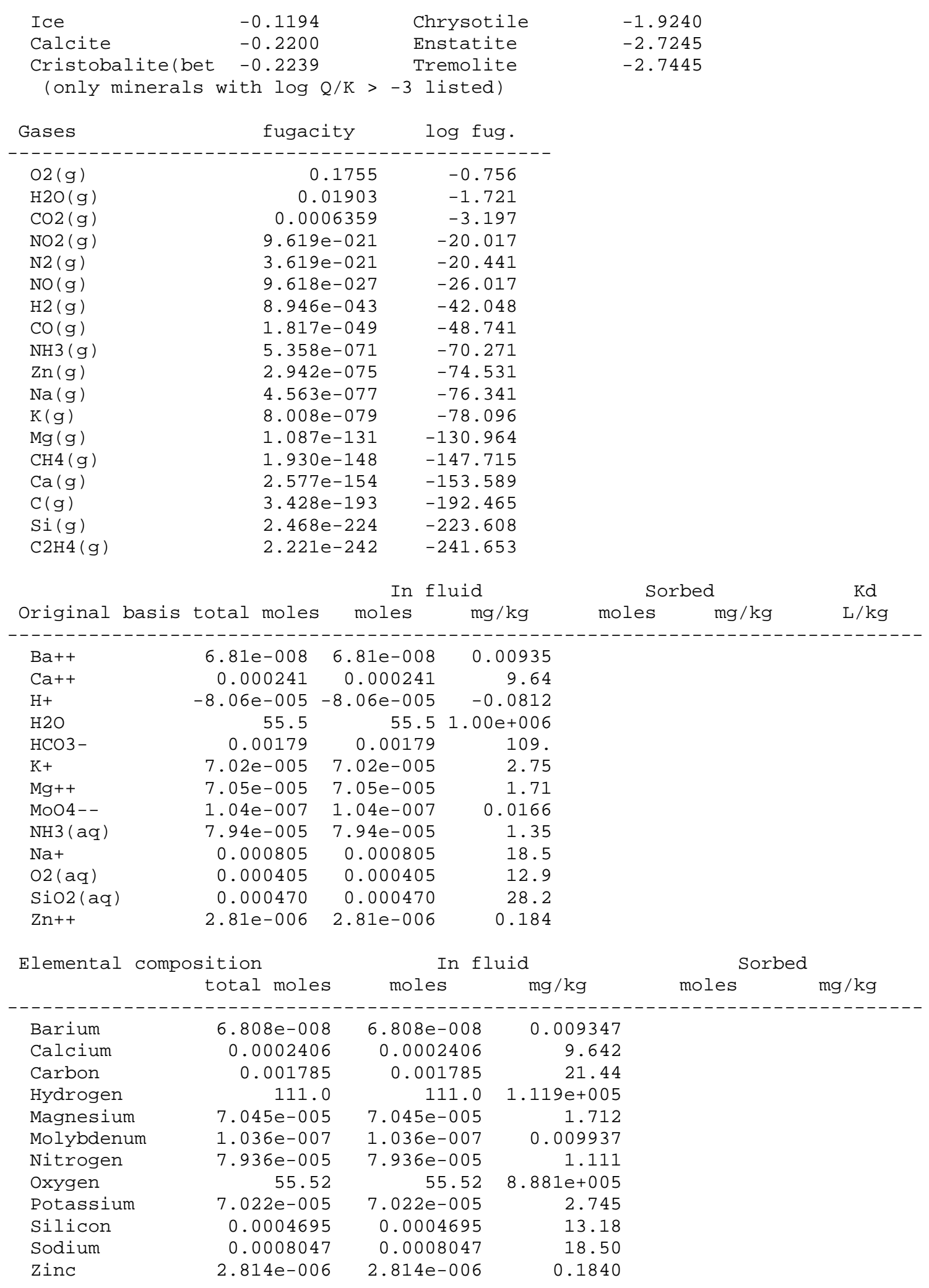




\section{Sample D11NEB0T}

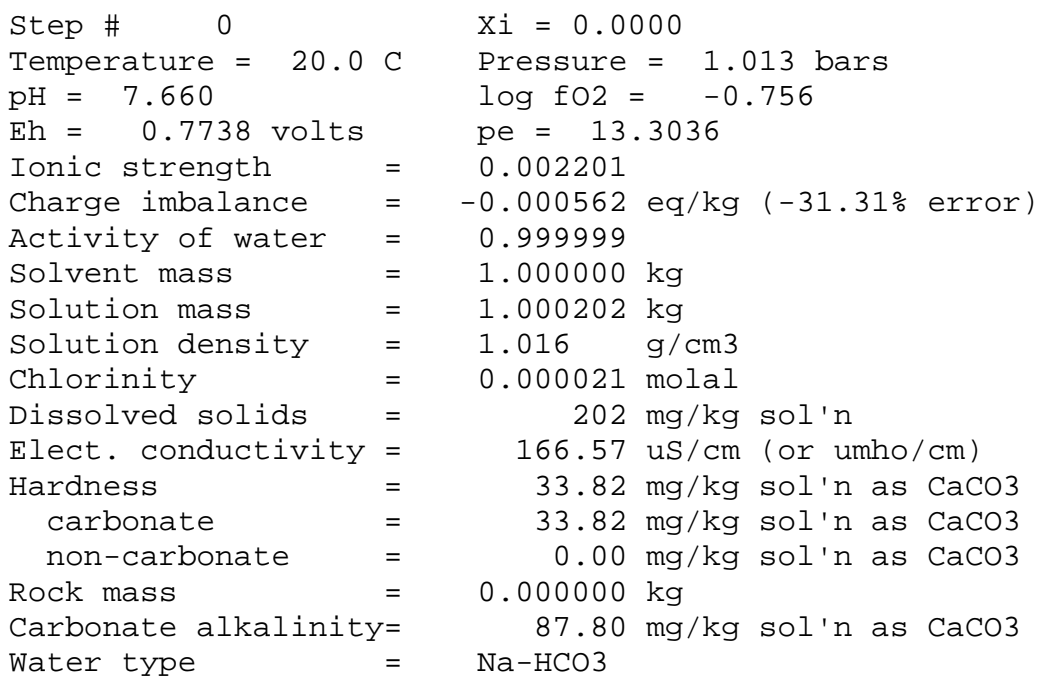

No minerals in system.

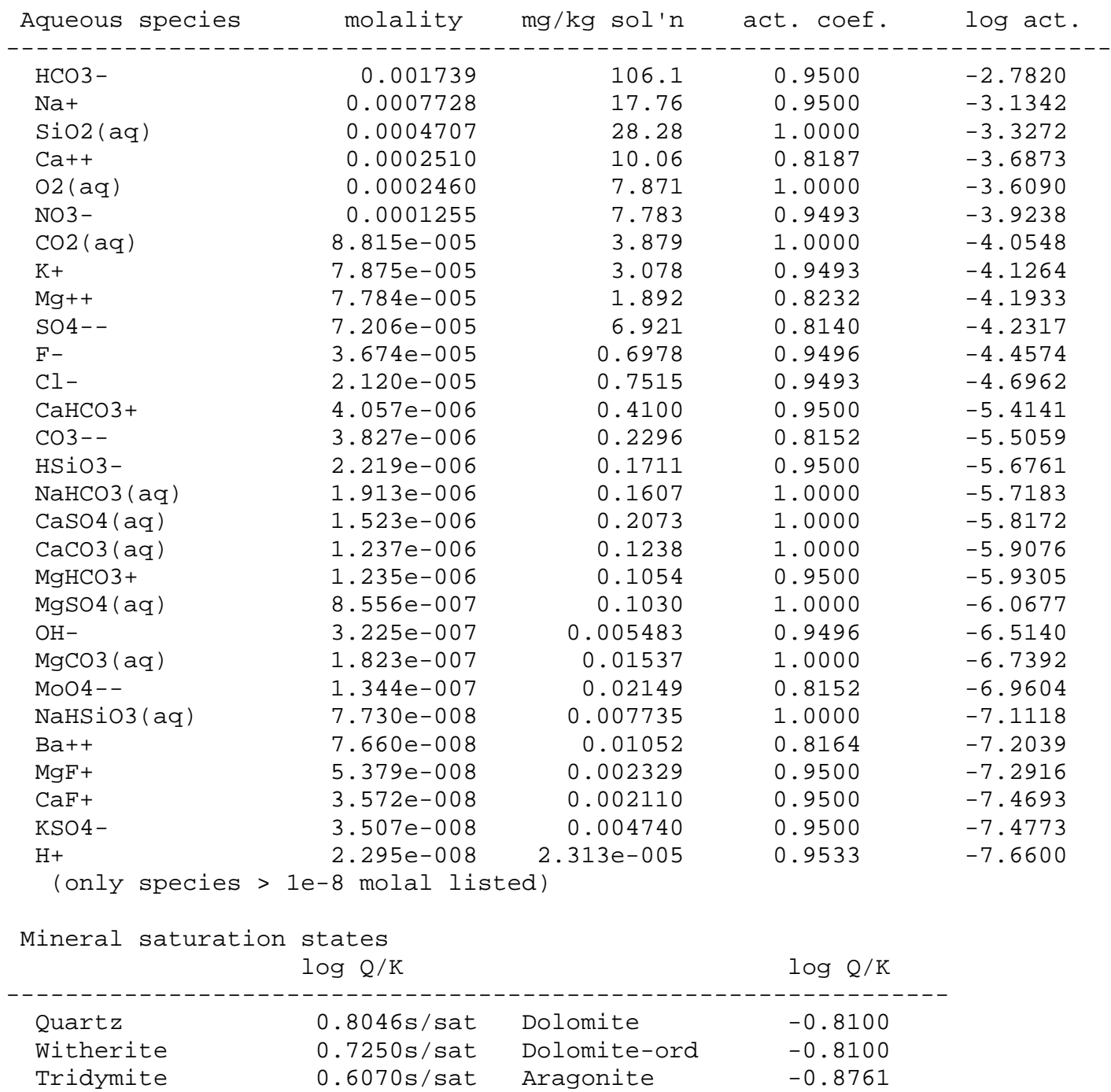




$\begin{array}{llll}\text { Cristobalite(alp } & 0.2426 \mathrm{~s} / \mathrm{sat} & \text { Barite } & -1.3692 \\ \text { Chalcedony } & 0.2287 \mathrm{~s} / \mathrm{sat} & \text { Talc } & -1.3878 \\ \text { Coesite } & -0.0186 & \text { Monohydrocalcite } & -1.5523 \\ \text { Ice } & -0.1194 & \text { Magnesite } & -1.7385 \\ \text { Cristobalite(bet } & -0.2155 & \text { Dolomite-dis } & -2.3918 \\ \text { Sio2(am) } & -0.5235 & \text { Fluorite } & -2.5150 \\ \text { Calcite } & -0.7315 & & \\ \quad \text { (only minerals with } \log \mathrm{Q} / \mathrm{K}>-3 \text { listed) } & \end{array}$

\begin{tabular}{lcc} 
Gases & fugacity & log fug \\
\hline O2 $(g)$ & 0.1755 & -0.756 \\
H2O $(\mathrm{g})$ & 0.01903 & -1.721 \\
$\mathrm{C} 2(\mathrm{~g})$ & 0.002205 & -2.657 \\
$\mathrm{HF}(\mathrm{g})$ & $3.858 \mathrm{e}-014$ & -13.414 \\
$\mathrm{HCl}(\mathrm{g})$ & $1.307 \mathrm{e}-019$ & -18.884 \\
$\mathrm{~N} 2(\mathrm{~g})$ & $1.083 \mathrm{e}-019$ & -18.965 \\
$\mathrm{NO}(\mathrm{g})$ & $5.263 \mathrm{e}-020$ & -19.279 \\
$\mathrm{NO}(\mathrm{g})$ & $5.263 \mathrm{e}-026$ & -25.279 \\
$\mathrm{C} 12(\mathrm{~g})$ & $2.104 \mathrm{e}-030$ & -29.677 \\
$\mathrm{SiF} 4(\mathrm{~g})$ & $2.105 \mathrm{e}-037$ & -36.677 \\
$\mathrm{H} 2(\mathrm{~g})$ & $8.946 \mathrm{e}-043$ & -42.048 \\
$\mathrm{CO}(\mathrm{g})$ & $6.300 \mathrm{e}-049$ & -48.201 \\
$\mathrm{~S} 02(\mathrm{~g})$ & $3.654 \mathrm{e}-057$ & -56.437 \\
$\mathrm{NH}(\mathrm{g})$ & $2.932 \mathrm{e}-070$ & -69.533 \\
$\mathrm{Na}(\mathrm{g})$ & $1.264 \mathrm{e}-077$ & -76.898 \\
$\mathrm{~K}(\mathrm{~g})$ & $2.583 \mathrm{e}-079$ & -78.588 \\
$\mathrm{~F} 2(\mathrm{~g})$ & $1.181 \mathrm{e}-083$ & -82.928 \\
$\mathrm{Mg}(\mathrm{g})$ & $1.014 \mathrm{e}-132$ & -131.994 \\
$\mathrm{H} 2 \mathrm{~g}(\mathrm{~g})$ & $5.179 \mathrm{e}-146$ & -145.286 \\
$\mathrm{CH} 4(\mathrm{~g})$ & $6.689 \mathrm{e}-148$ & -147.175 \\
$\mathrm{Ca}(\mathrm{g})$ & $2.289 \mathrm{e}-155$ & -154.640 \\
$\mathrm{C}(\mathrm{g})$ & $1.188 \mathrm{e}-192$ & -191.925 \\
$\mathrm{Si}(\mathrm{g})$ & $2.516 \mathrm{e}-224$ & -223.599 \\
$\mathrm{~S} 2(\mathrm{~g})$ & $2.271 \mathrm{e}-233$ & -232.644 \\
$\mathrm{C} 2 \mathrm{H} 4(\mathrm{~g})$ & $2.670 \mathrm{e}-241$ & -240.574 \\
& &
\end{tabular}

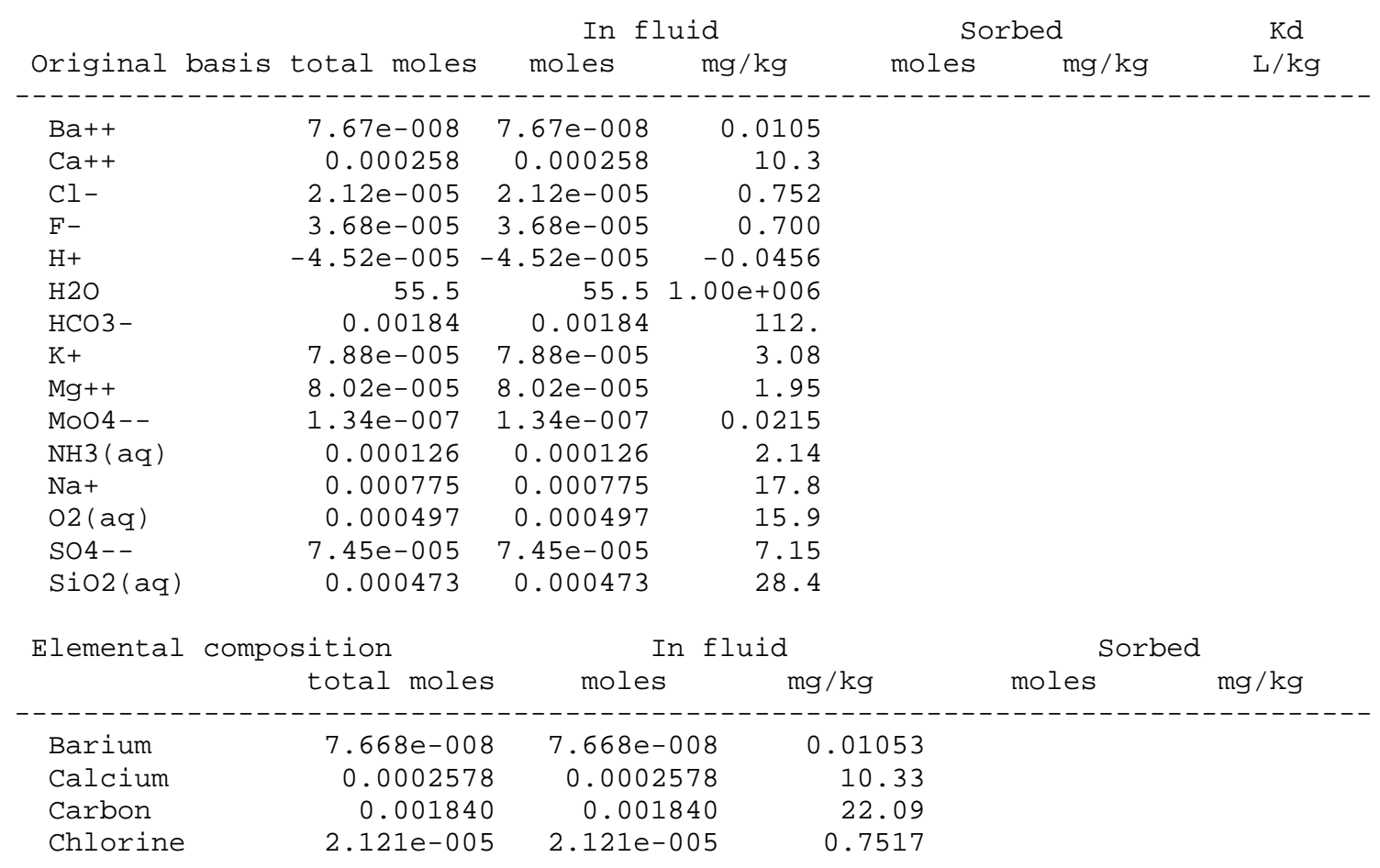




$\begin{array}{lrrr}\text { Fluorine } & 3.683 \mathrm{e}-005 & 3.683 \mathrm{e}-005 & 0.6995 \\ \text { Hydrogen } & 111.0 & 111.0 & 1.119 \mathrm{e}+005 \\ \text { Magnesium } & 8.017 \mathrm{e}-005 & 8.017 \mathrm{e}-005 & 1.948 \\ \text { Molybdenum } & 1.344 \mathrm{e}-007 & 1.344 \mathrm{e}-007 & 0.01289 \\ \text { Nitrogen } & 0.0001255 & 0.0001255 & 1.758 \\ \text { Oxygen } & 55.52 & 55.52 & 8.880 \mathrm{e}+005 \\ \text { Potassium } & 7.878 \mathrm{e}-005 & 7.878 \mathrm{e}-005 & 3.080 \\ \text { Silicon } & 0.0004730 & 0.0004730 & 13.28 \\ \text { Sodium } & 0.0007748 & 0.0007748 & 17.81 \\ \text { Sulfur } & 7.447 \mathrm{e}-005 & 7.447 \mathrm{e}-005 & 2.388\end{array}$




\section{Sample D11NWB0T}

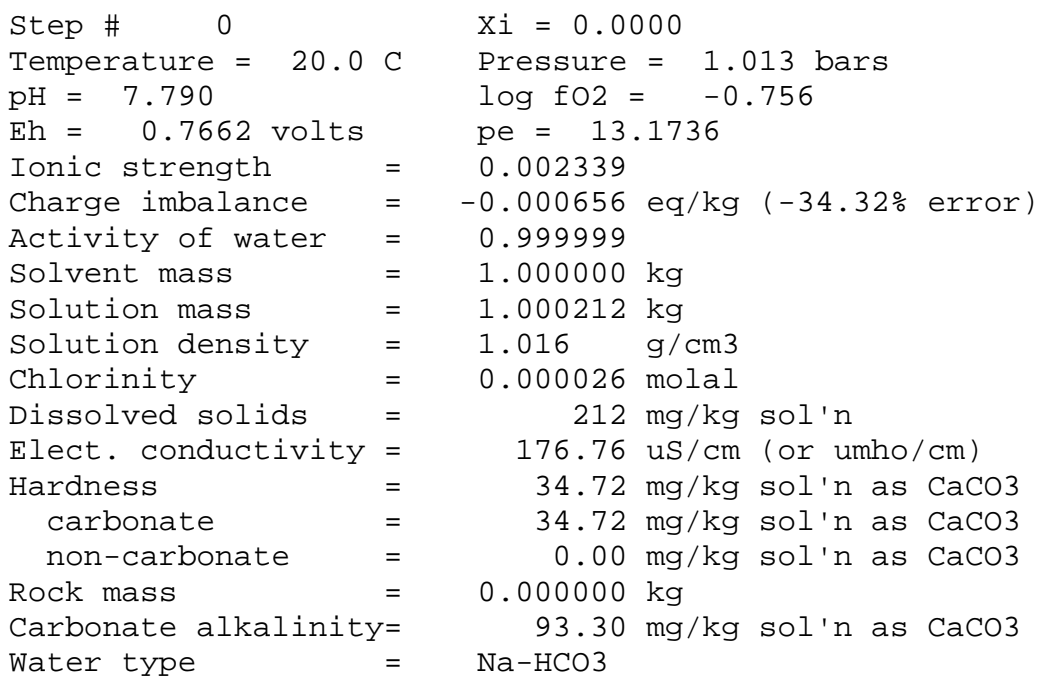

No minerals in system.

\begin{tabular}{|c|c|c|c|c|}
\hline Aqueous species & molality & $\mathrm{mg} / \mathrm{kg}$ sol'n & act. coef. & $\log$ act \\
\hline$-8--------1$ & & - - - - - - - - - & 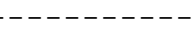 & ---- \\
\hline $\mathrm{HCO} 3-$ & $\odot .001844$ & 112.5 & $\odot .9486$ & -2.7571 \\
\hline $\mathrm{Na}+$ & $\odot .0 \odot \odot 8239$ & 18.94 & $\odot .9486$ & -3.1071 \\
\hline $\operatorname{SiO} 2(\mathrm{aq})$ & $\odot .0 \odot \odot 449 \odot$ & 26.97 & 1.0000 & -3.3477 \\
\hline $\mathrm{Ca}++$ & ๑. $00 \odot 2525$ & 10.12 & 0.8141 & -3.6871 \\
\hline $02(\mathrm{aq})$ & 0.0002460 & 7.871 & 1.0000 & -3.6090 \\
\hline N03 - & ๑. . $0 \odot 1532$ & 9.495 & $\odot .9478$ & -3.8381 \\
\hline $\mathrm{Mg}++$ & 8. 388e- -๑५ & 2.038 & $\odot .8188$ & -4.1632 \\
\hline s04 - - & $8.140 \mathrm{e}-005$ & 7.818 & 0.8091 & -4.1814 \\
\hline $\mathrm{K}+$ & $7.698 e-0 \odot 5$ & 3.009 & $\odot .9478$ & -4.1369 \\
\hline $\mathrm{co2}(\mathrm{aq})$ & $6.920 \mathrm{e}-005$ & 3.045 & 1.0000 & -4.1599 \\
\hline $\mathrm{F}-$ & $3.389 e-005$ & $\odot .6437$ & 0.9482 & -4.4930 \\
\hline $\mathrm{Cl}-$ & $2.606 \mathrm{e}-\odot \odot 5$ & $\odot .9237$ & $\odot .9478$ & -4.6073 \\
\hline CO3-- & $5.499 e-0 \odot 6$ & ๑. 3299 & $\odot .8104$ & -5.3511 \\
\hline Re04 - & $5.338 e-0 \odot 6$ & 1.335 & $\odot .9486$ & -5.2956 \\
\hline CaHCO3+ & $4.3 \odot 3 e-\odot \odot 6$ & $\odot .4350$ & $\odot .9486$ & -5.3891 \\
\hline HSiO3 - & $2.860 \mathrm{e}-006$ & 0.2204 & $\odot .9486$ & -5.5665 \\
\hline Zn++ & $2.474 \mathrm{e}-006$ & 0.1617 & 0.8141 & -5.6960 \\
\hline $\mathrm{NaHCO} 3(\mathrm{aq})$ & $2.156 \mathrm{e}-0 \odot 6$ & 0.1811 & 1.0000 & -5.6663 \\
\hline $\mathrm{CaCO} 3(\mathrm{aq})$ & $1.768 \mathrm{e}-006$ & 0.1769 & 1.0000 & -5.7526 \\
\hline CaSO4(aq) & $1.711 \mathrm{e}-006$ & $\odot .2329$ & 1.0000 & -5.7667 \\
\hline $\mathrm{MgHCO} 3+$ & $1.404 \mathrm{e}-006$ & 0.1198 & 0.9486 & -5.8755 \\
\hline $\operatorname{MgSO} 4(\mathrm{aq})$ & 1. $.30 \mathrm{e}-0 \odot 6$ & 0.1239 & 1.0000 & -5.9872 \\
\hline Mo04-- & $5.560 \mathrm{e}-\odot \odot 7$ & ๑. $\odot 8890$ & 0.8104 & -6.3463 \\
\hline $\mathrm{OH}-$ & $4.356 e-0 \odot 7$ & $\odot .007408$ & 0.9482 & -6.3840 \\
\hline $\operatorname{Mgc03}(\mathrm{aq})$ & $2.791 \mathrm{e}-0 \odot 7$ & 0.02353 & $1.000 \odot$ & -6.5542 \\
\hline NaHSiO3(aq) & $1.059 \mathrm{e}-007$ & 0.01060 & 1.0000 & -6.9751 \\
\hline $\mathrm{Ba}++$ & $7.728 e-\odot \odot 8$ & ๑. . 01061 & $\odot .8116$ & -7.2026 \\
\hline $\mathrm{MgF}+$ & $5.319 e-\odot \odot 8$ & ๑ . $0 \odot 2303$ & $\odot .9486$ & -7.2971 \\
\hline KSO4 - & $3.850 \mathrm{e}-\odot \odot 8$ & ๑. . 005203 & $\odot .9486$ & -7.4375 \\
\hline CaF+ & $3.297 e-0 \odot 8$ & $\odot .001947$ & 0.9486 & -7.5049 \\
\hline Zns04(aq) & $2.455 \mathrm{e}-0 \odot 8$ & $\odot .003963$ & 1.0000 & -7.6100 \\
\hline $\mathrm{H}+$ & 1. $703 \mathrm{e}-0 \odot 8$ & $1.717 \mathrm{e}-0 \odot 5$ & $\odot .9521$ & -7.7900 \\
\hline $\mathrm{NaCO} 3-$ & $1.386 e-008$ & ๑. 001150 & $\odot .9486$ & -7.8812 \\
\hline
\end{tabular}




\begin{tabular}{|c|c|c|c|}
\hline & $\log Q / K$ & & $\log Q / K$ \\
\hline Zn2Si04 & $2.2053 \mathrm{~s} / \mathrm{sat}$ & Talc & -0.5994 \\
\hline Witherite & $\odot .8812 \mathrm{~s} / \mathrm{sat}$ & Aragonite & -0.7211 \\
\hline Quartz & $\odot .7841 \mathrm{~s} / \mathrm{sat}$ & Smithsonite & -1.2140 \\
\hline Tridymite & $\odot .5865 \mathrm{~s} / \mathrm{sat}$ & Barite & -1.3175 \\
\hline Cristobalite(alp & $0.2221 \mathrm{~s} / \mathrm{sat}$ & Monohydrocalcite & -1.3973 \\
\hline Chalcedony & $0.2082 \mathrm{~s} / \mathrm{sat}$ & Magnesite & -1.5535 \\
\hline Coesite & -0.0391 & Zincite & -1.5945 \\
\hline Ice & -0.1194 & $\mathrm{Zn}(\mathrm{OH}) 2($ gamma $)$ & -1.9992 \\
\hline Cristobalite(bet & -0.2359 & $\mathrm{Zn}(\mathrm{OH}) 2$ (epsilon) & -2.0245 \\
\hline Dolomite & -0.4700 & Dolomite-dis & -2.0518 \\
\hline Dolomite-ord & -0.4700 & $\mathrm{Zn}(\mathrm{OH}) 2$ (beta) & -2.3004 \\
\hline $\mathrm{SiO} 2(\mathrm{am})$ & -0.5440 & Fluorite & -2.5862 \\
\hline Calcite & -0.5765 & & \\
\hline (only minerals & ith $\log Q / K>$ & 3 listed) & \\
\hline Gases & fugacity & $\log$ fug. & \\
\hline 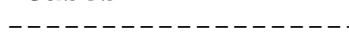 & $-\ldots-\ldots-\ldots$ & 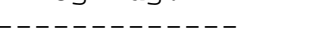 & \\
\hline $02(g)$ & 0.1755 & $-\odot .756$ & \\
\hline $\mathrm{H} 2 \mathrm{O}(\mathrm{g})$ & 0.01903 & -1.721 & \\
\hline $\mathrm{co2}(\mathrm{g})$ & 0.001731 & -2.762 & \\
\hline $\mathrm{HF}(\mathrm{g})$ & $2.635 e-014$ & -13.579 & \\
\hline $\mathrm{HCl}(\mathrm{g})$ & $1.189 \mathrm{e}-019$ & -18.925 & \\
\hline $\mathrm{N} 2(\mathrm{~g})$ & $8.834 \mathrm{e}-020$ & -19.054 & \\
\hline $\mathrm{N} 02(\mathrm{~g})$ & $4.753 \mathrm{e}-02 \odot$ & -19.323 & \\
\hline $\mathrm{NO}(\mathrm{g})$ & $4.752 \mathrm{e}-026$ & -25.323 & \\
\hline Cl2(g) & $1.741 \mathrm{e}-030$ & -29.759 & \\
\hline SiF4(g) & $4.366 e-038$ & -37.360 & \\
\hline $\mathrm{H} 2(\mathrm{~g})$ & $8.946 e-043$ & -42.048 & \\
\hline co(g) & $4.946 e-049$ & -48.306 & \\
\hline SO2(g) & $2.255 e-057$ & -56.647 & \\
\hline $\mathrm{NH3}(\mathrm{g})$ & $2.648 e-07 \odot$ & -69.577 & \\
\hline $\mathrm{Zn}(\mathrm{g})$ & $3.856 e-076$ & -75.414 & \\
\hline $\mathrm{Na}(\mathrm{g})$ & $1.816 \mathrm{e}-077$ & -76.741 & \\
\hline$K(g)$ & $3.402 \mathrm{e}-079$ & -78.468 & \\
\hline $\mathrm{F} 2(\mathrm{~g})$ & $5.508 e-084$ & -83.259 & \\
\hline $\operatorname{Mg}(g)$ & 1. $978 e-132$ & -131.704 & \\
\hline $\mathrm{H} 2 \mathrm{~S}(\mathrm{~g})$ & $3.196 \mathrm{e}-146$ & -145.495 & \\
\hline $\mathrm{CH} 4(\mathrm{~g})$ & $5.251 \mathrm{e}-148$ & -147.280 & \\
\hline $\mathrm{Ca}(\mathrm{g})$ & $4.167 e-155$ & -154.380 & \\
\hline$C(g)$ & $9.328 e-193$ & - 192.030 & \\
\hline $\mathrm{Si}(\mathrm{g})$ & $2.400 \mathrm{e}-224$ & -223.620 & \\
\hline $\mathrm{s} 2(\mathrm{~g})$ & $8.646 e-234$ & -233.063 & \\
\hline $\mathrm{C} 2 \mathrm{H} 4(\mathrm{~g})$ & $1.645 e-241$ & -240.784 & \\
\hline
\end{tabular}

\begin{tabular}{|c|c|c|c|c|c|}
\hline \multirow{3}{*}{\multicolumn{2}{|c|}{ Original basis total moles }} & \multicolumn{2}{|c|}{ In fluid } & Sorbed & \multirow{2}{*}{$\begin{array}{c}\mathrm{Kd} \\
\mathrm{L} / \mathrm{kg}\end{array}$} \\
\hline & & moles & $\mathrm{mg} / \mathrm{kg}$ & moles $\quad \mathrm{mg} / \mathrm{kg}$ & \\
\hline & & -------- & $--\ldots$ & & \\
\hline $\mathrm{Ba}++$ & $7.74 \mathrm{e}-\odot \odot 8$ & $7.74 \mathrm{e}-\odot \odot 8$ & ๑. 0106 & & \\
\hline $\mathrm{Ca}++$ & 0.000260 & 0.000260 & 10.4 & & \\
\hline $\mathrm{Cl}-$ & $2.61 \mathrm{e}-005$ & $2.61 \mathrm{e}-005$ & $\odot .924$ & & \\
\hline $\mathrm{F}-$ & $3.40 \mathrm{e}-005$ & $3.40 e-005$ & 0.645 & & \\
\hline $\mathrm{H}+$ & $-9.49 e-005$ & $-9.49 e-005$ & -0.0956 & & \\
\hline $\mathrm{H} 2 \mathrm{O}$ & 55.5 & 55.5 & $1.00 \mathrm{e}+\odot \odot 6$ & & \\
\hline HCO3 - & $\odot .00193$ & $\odot .0 \odot 193$ & 118 & & \\
\hline $\mathrm{K}+$ & $7.70 \mathrm{e}-005$ & $7.70 \mathrm{e}-005$ & 3.01 & & \\
\hline $\mathrm{Mg}++$ & $8.66 e-005$ & $8.66 e-005$ & 2.11 & & \\
\hline Mo04 - - & $5.56 e-007$ & $5.56 e-007$ & ๑. 0889 & & \\
\hline $\mathrm{NH3}(\mathrm{aq})$ & ๑. . 000153 & ๑. . 000153 & 2.61 & & \\
\hline $\mathrm{Na}+$ & $\odot .000826$ & 0.000826 & 19.0 & & \\
\hline $02(\mathrm{aq})$ & 0.000552 & $\odot .000552$ & 17.7 & & \\
\hline
\end{tabular}




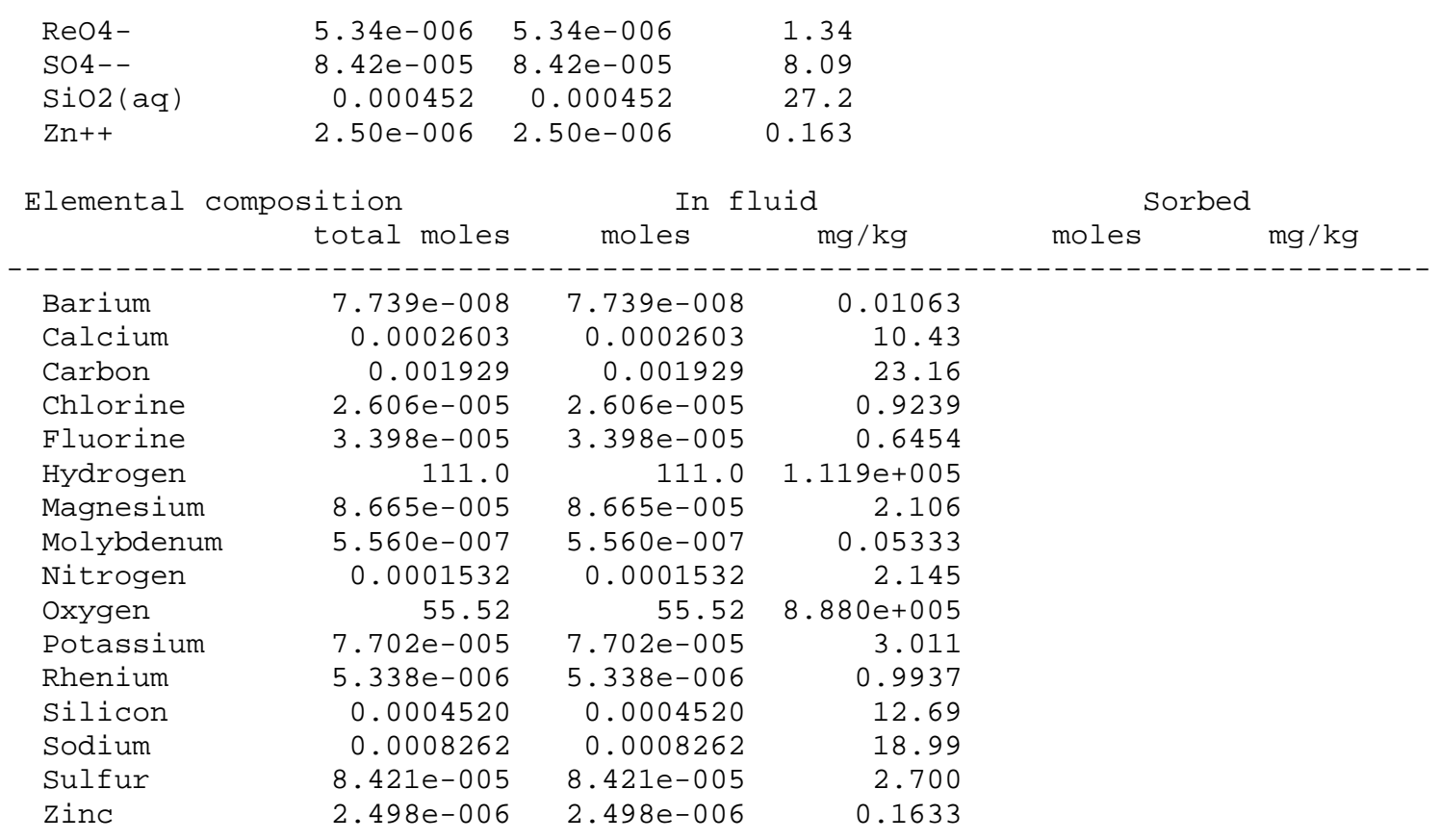




\section{Sample D11SEB0T}

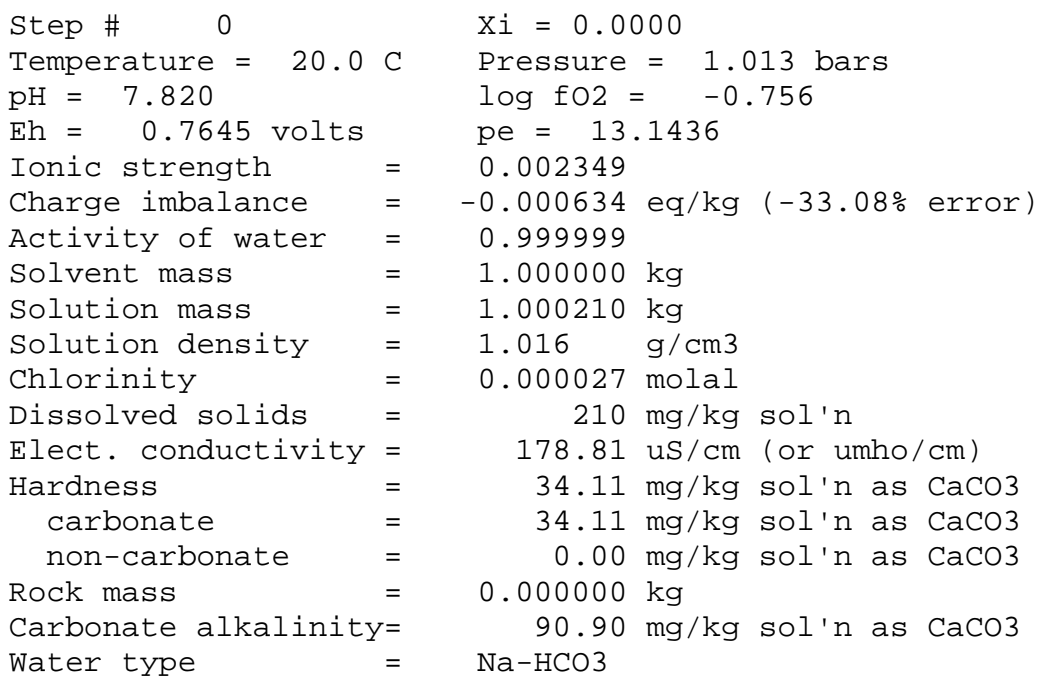

No minerals in system.

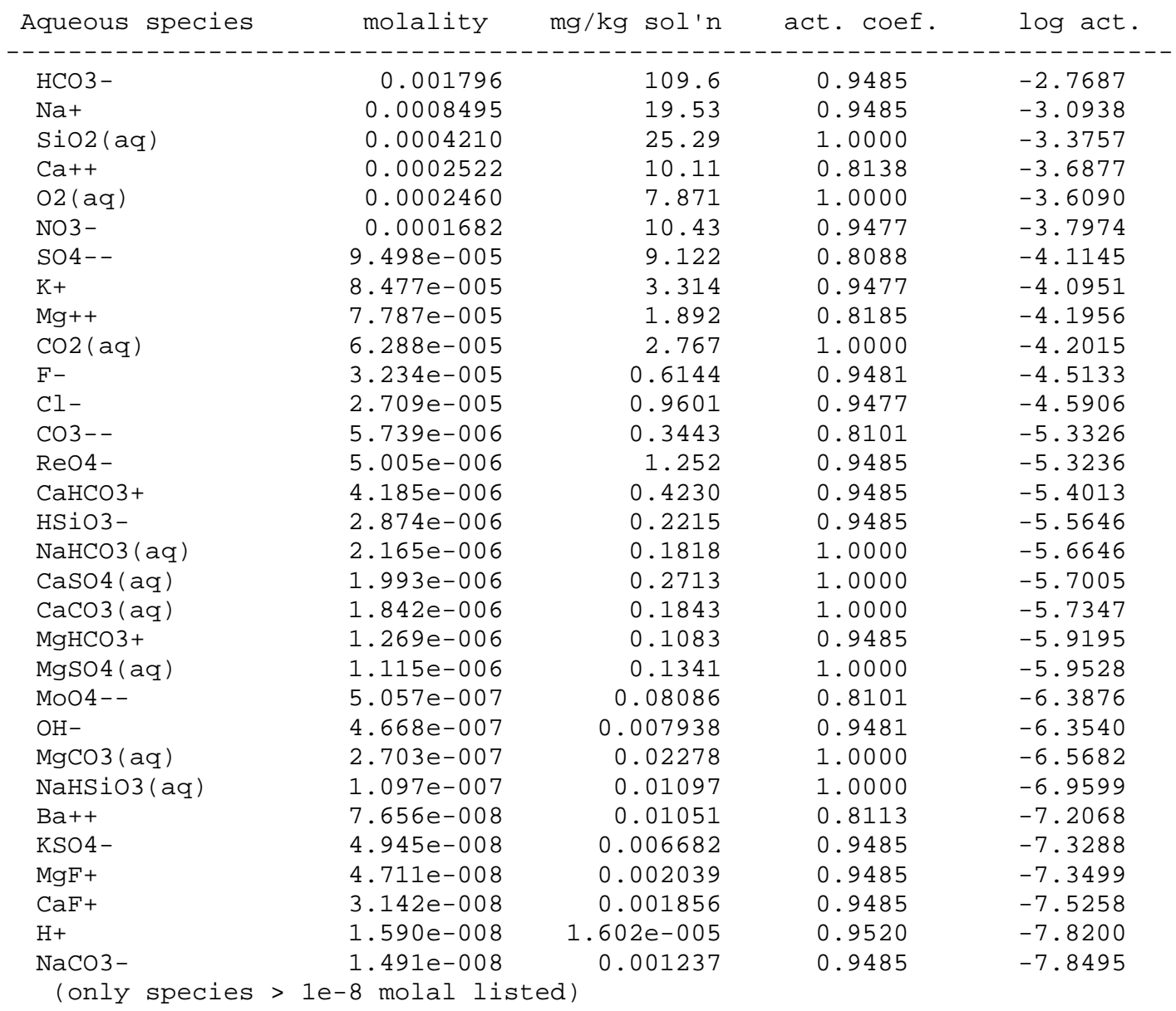




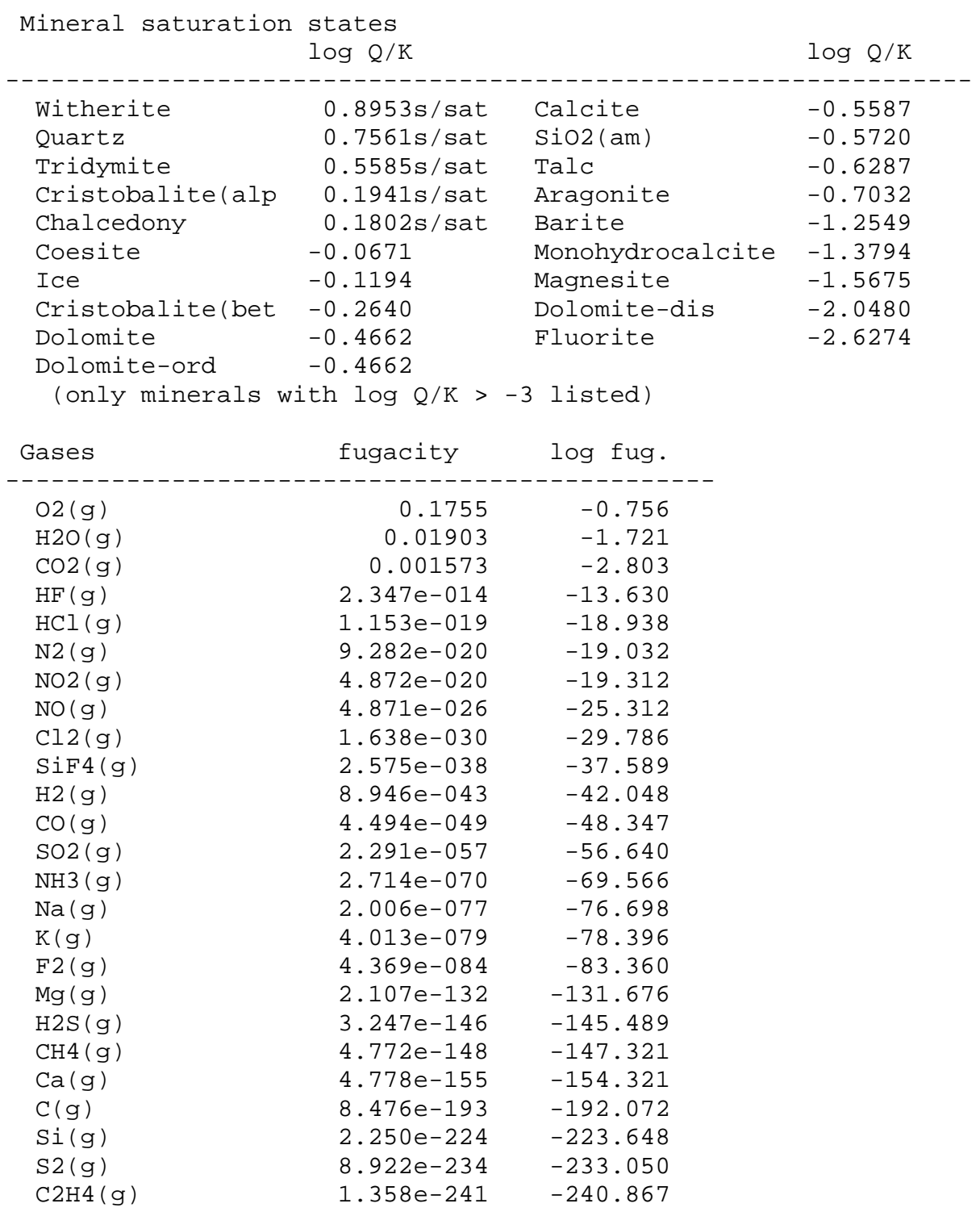

\begin{tabular}{|c|c|c|c|c|c|}
\hline \multirow[b]{2}{*}{ Original basis } & \multirow[b]{2}{*}{ total moles } & \multicolumn{2}{|c|}{ In fluid } & Sorbed & \multirow{2}{*}{$\begin{array}{c}\mathrm{Kd} \\
\mathrm{L} / \mathrm{kg}\end{array}$} \\
\hline & & moles & $\mathrm{mg} / \mathrm{kg}$ & moles $\quad \mathrm{mg} / \mathrm{kg}$ & \\
\hline - & - - - - - - - - - & $-\ldots-\ldots$ & -------1 & - - - - - - - - - - & \\
\hline $\mathrm{Ba}++$ & $7.67 \mathrm{e}-0 \odot 8$ & $7.67 e-0 \odot 8$ & $\odot .0105$ & & \\
\hline $\mathrm{Ca}++$ & 0.000260 & $\odot .000260$ & 10.4 & & \\
\hline Cl- & $2.71 e-005$ & $2.71 e-005$ & 0.960 & & \\
\hline $\mathrm{F}-$ & $3.24 \mathrm{e}-0 \odot 5$ & $3.24 \mathrm{e}-\odot \odot 5$ & $\odot .616$ & & \\
\hline $\mathrm{H}+$ & -0.000117 & -0.000117 & -0.118 & & \\
\hline $\mathrm{H} 2 \mathrm{O}$ & 55.5 & 55.5 & $1.00 \mathrm{e}+006$ & & \\
\hline $\mathrm{HCO} 3-$ & 0.00187 & 0.00187 & 114 & & \\
\hline $\mathrm{K}+$ & $8.48 e-005$ & $8.48 e-005$ & 3.32 & & \\
\hline $\mathrm{Mg}++$ & $8.06 e-005$ & $8.06 e-005$ & 1.96 & & \\
\hline Mo04 - - & $5.06 e-007$ & $5.06 e-007$ & 0.0809 & & \\
\hline $\mathrm{NH} 3(\mathrm{aq})$ & ๑. 000168 & 0.000168 & 2.86 & & \\
\hline $\mathrm{Na}+$ & $\odot .00 \odot 852$ & $\odot .000852$ & 19.6 & & \\
\hline $02(\mathrm{aq})$ & 0.000583 & 0.000583 & 18.6 & & \\
\hline $\mathrm{ReO4-}$ & $5.0 \odot \mathrm{e}-\odot \odot 6$ & 5. $\odot \odot e-\odot \odot 6$ & 1.25 & & \\
\hline SO4 - - & $9.81 e-005$ & $9.81 e-005$ & 9.43 & & \\
\hline Si02(aq) & 0.000424 & $\odot .000424$ & 25.5 & & \\
\hline
\end{tabular}




\begin{tabular}{|c|c|c|c|c|c|}
\hline \multicolumn{2}{|c|}{ Elemental composition } & \multicolumn{2}{|c|}{ In fluid } & \multicolumn{2}{|c|}{ Sorbed } \\
\hline & total moles & moles & $\mathrm{mg} / \mathrm{kg}$ & moles & $\mathrm{mg} / \mathrm{kg}$ \\
\hline 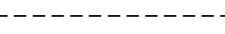 & - - - - - - - - - & - - - - - - - - - & 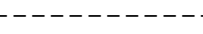 & ---- & \\
\hline Barium & $7.668 e-\odot \odot 8$ & $7.668 e-\odot \odot 8$ & ๑. 01053 & & \\
\hline Calcium & $\odot .0002603$ & 0.0002603 & 10.43 & & \\
\hline Carbon & ๑. . 001874 & ๑. . 001874 & 22.51 & & \\
\hline Chlorine & $2.709 e-005$ & $2.709 \mathrm{e}-005$ & 0.9603 & & \\
\hline Fluorine & $3.243 e-005$ & $3.243 e-005$ & ๑.6159 & & \\
\hline Hydrogen & 111.0 & 111.0 & $1.119 \mathrm{e}+005$ & & \\
\hline Magnesium & $8.057 e-005$ & $8.057 e-005$ & 1.958 & & \\
\hline Molybdenum & $5.057 e-007$ & $5.057 e-007$ & $\odot .04851$ & & \\
\hline Nitrogen & ๑. $0 \odot \odot 1682$ & $\odot .0 \odot \odot 1682$ & 2.356 & & \\
\hline oxygen & 55.52 & 55.52 & $8.880 e+005$ & & \\
\hline Potassium & $8.482 \mathrm{e}-005$ & $8.482 \mathrm{e}-005$ & 3.316 & & \\
\hline Rhenium & $5.005 e-006$ & $5.0 \odot 5 e-\odot \odot 6$ & 0.9317 & & \\
\hline Silicon & $\odot .0 \odot \odot 424 \odot$ & $\odot .0 \odot \odot 424 \odot$ & 11.91 & & \\
\hline Sodium & 0.0008518 & 0.0008518 & 19.58 & & \\
\hline Sulfur & $9.814 \mathrm{e}-005$ & $9.814 e-005$ & 3.146 & & \\
\hline
\end{tabular}




\section{Sample D11SWB0T}

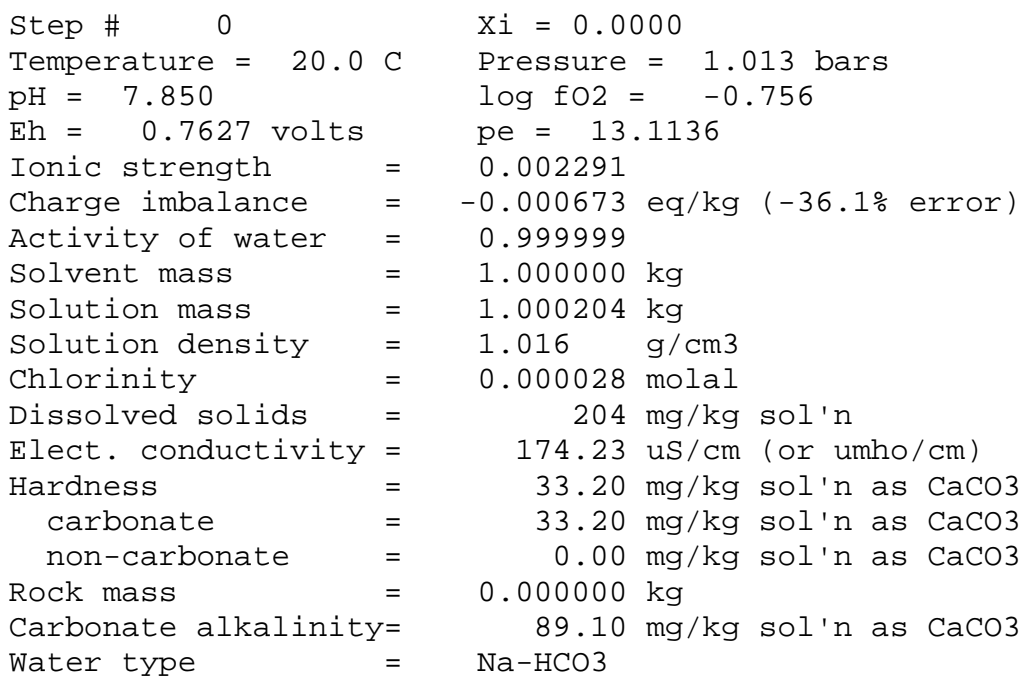

No minerals in system.

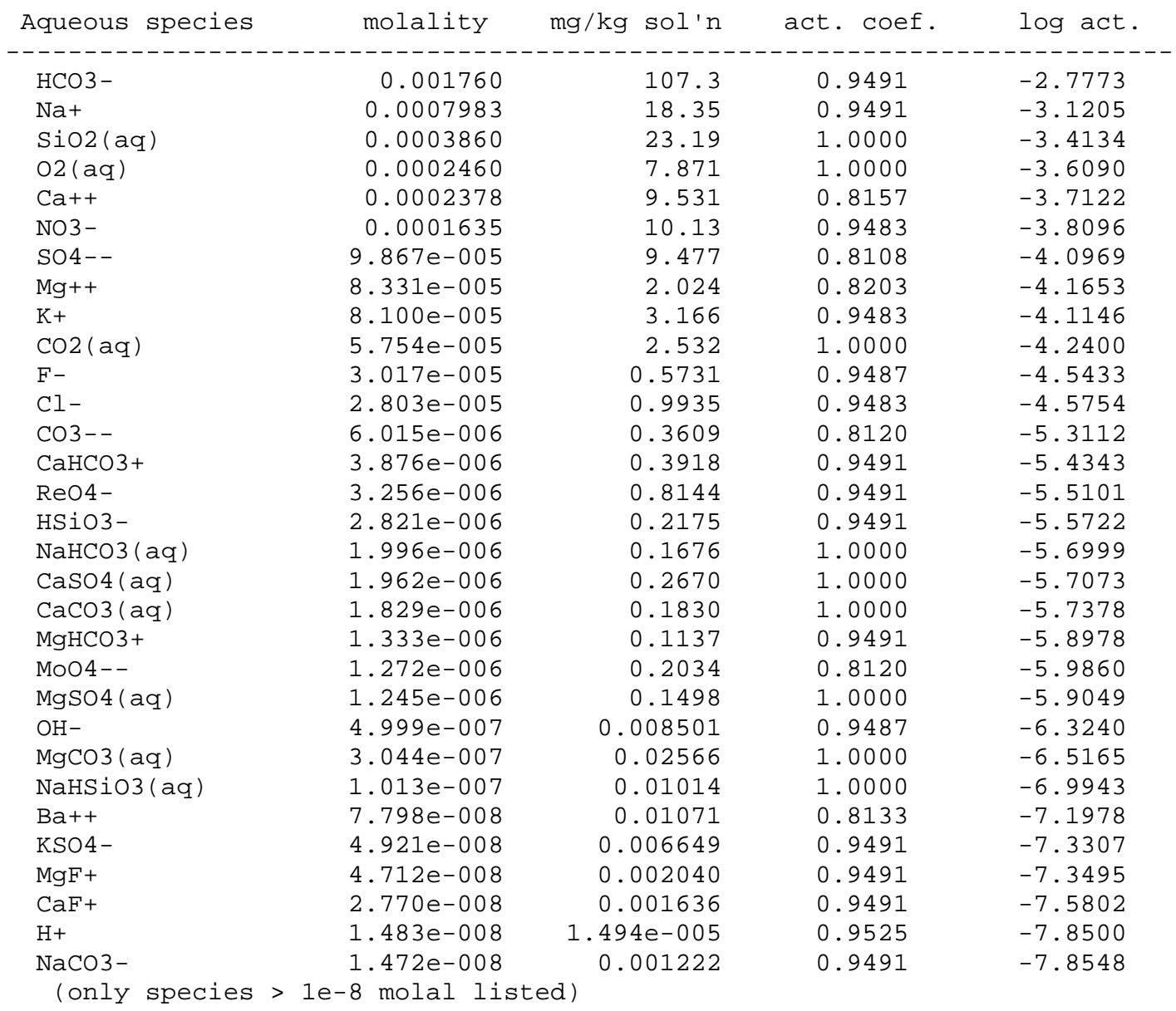




\begin{tabular}{|c|c|c|c|}
\hline Mineral saturatio & \multicolumn{2}{|c|}{$\log \mathrm{Q} / \mathrm{K}$} & $\log Q / K$ \\
\hline \multicolumn{4}{|c|}{ - - - - - - - - - - - - - - - - - - - - - - - - - - - - - - - - - - - - - - } \\
\hline Witherite & $\odot .9258 \mathrm{~s} / \mathrm{sat}$ & Talc & $-\odot .5086$ \\
\hline Quartz & $\odot .7184 \mathrm{~s} / \mathrm{sat}$ & Calcite & -0.5617 \\
\hline Tridymite & $0.5208 \mathrm{~s} / \mathrm{sat}$ & Si02( am ) & -0.6097 \\
\hline Cristobalite(alp & $0.1564 \mathrm{~s} / \mathrm{sat}$ & Aragonite & -0.7063 \\
\hline Chalcedony & $0.1425 \mathrm{~s} / \mathrm{sat}$ & Barite & -1.2282 \\
\hline Coesite & 0.1048 & Monohydrocalcite & -1.3825 \\
\hline Ice & $\odot .1194$ & Magnesite & -1.5158 \\
\hline Cristobalite(bet & 0.3016 & Dolomite-dis & -1.9993 \\
\hline Dolomite & 0.4175 & Fluorite & -2.7117 \\
\hline $\begin{array}{l}\text { Dolomite-ord } \\
\text { (only minerals }\end{array}$ & $\begin{array}{l}0.4175 \\
h \log Q / K>\end{array}$ & 3 listed) & \\
\hline Gases & fugacity & $\log$ fug. & \\
\hline \multicolumn{4}{|c|}{ 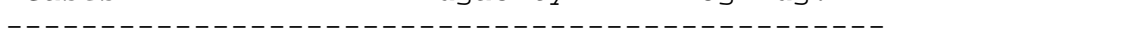 } \\
\hline $02(\mathrm{~g})$ & 0.1755 & $-\odot .756$ & \\
\hline $\mathrm{H} 2 \mathrm{O}(\mathrm{g})$ & $\odot .01903$ & -1.721 & \\
\hline $\mathrm{co2}(\mathrm{g})$ & 0.001439 & -2.842 & \\
\hline$H F(g)$ & $2.044 \mathrm{e}-014$ & -13.689 & \\
\hline $\mathrm{HCl}(\mathrm{g})$ & $1.114 \mathrm{e}-019$ & -18.953 & \\
\hline N2 $(g)$ & $7.643 e-02 \odot$ & -19.117 & \\
\hline N02(g) & $4.421 \mathrm{e}-020$ & -19.355 & \\
\hline NO $(g)$ & $4.42 \odot e-026$ & -25.355 & \\
\hline Cl2(g) & $1.530 \mathrm{e}-030$ & -29.815 & \\
\hline $\operatorname{SiF} 4(g)$ & $1.360 \mathrm{e}-038$ & -37.867 & \\
\hline $\mathrm{H} 2(\mathrm{~g})$ & $8.946 e-043$ & -42.048 & \\
\hline $\mathrm{CO}(\mathrm{g})$ & $4.112 \mathrm{e}-049$ & -48.386 & \\
\hline S02(g) & $2.078 e-057$ & -56.682 & \\
\hline NH3 $(\mathrm{g})$ & $2.463 e-070$ & -69.609 & \\
\hline $\mathrm{Na}(\mathrm{g})$ & $2.021 \mathrm{e}-077$ & -76.694 & \\
\hline$K(g)$ & $4.112 \mathrm{e}-079$ & -78.386 & \\
\hline F2(g) & $3.315 e-084$ & -83.479 & \\
\hline $\operatorname{Mg}(\mathrm{g})$ & $2.594 \mathrm{e}-132$ & -131.586 & \\
\hline $\mathrm{H} 2 \mathrm{~S}(\mathrm{~g})$ & $2.945 e-146$ & -145.531 & \\
\hline $\mathrm{CH} 4(\mathrm{~g})$ & $4.366 e-148$ & -147.360 & \\
\hline $\mathrm{Ca}(\mathrm{g})$ & $5.185 e-155$ & -154.285 & \\
\hline$C(g)$ & $7.756 \mathrm{e}-193$ & -192.110 & \\
\hline $\operatorname{si}(g)$ & $2.063 e-224$ & -223.685 & \\
\hline $\mathrm{S} 2(\mathrm{~g})$ & $7.341 e-234$ & -233.134 & \\
\hline $\mathrm{C} 2 \mathrm{H} 4(\mathrm{~g})$ & $1.137 e-241$ & -240.944 & \\
\hline
\end{tabular}

\begin{tabular}{|c|c|c|c|c|c|}
\hline \multirow[b]{2}{*}{ Original basis } & \multirow[b]{2}{*}{ total moles } & \multicolumn{2}{|c|}{ In fluid } & Sorbed & \multirow{2}{*}{$\begin{array}{c}\mathrm{Kd} \\
\mathrm{L} / \mathrm{kg}\end{array}$} \\
\hline & & moles & $\mathrm{mg} / \mathrm{kg}$ & moles $\quad \mathrm{mg} / \mathrm{kg}$ & \\
\hline $\mathrm{Ba}++$ & $7.81 e-008$ & $7.81 \mathrm{e}-008$ & 0.0107 & & \\
\hline $\mathrm{Ca}++$ & 0.000246 & 0.000246 & 9.84 & & \\
\hline $\mathrm{Cl}-$ & $2.80 e-005$ & $2.80 \mathrm{e}-005$ & $\odot .994$ & & \\
\hline $\mathrm{F}-$ & $3.03 e-005$ & $3.03 e-005$ & 0.575 & & \\
\hline $\mathrm{H}+$ & -0.000118 & -0.000118 & -0.118 & & \\
\hline $\mathrm{H} 2 \mathrm{O}$ & 55.5 & 55.5 & $1.00 \mathrm{e}+006$ & & \\
\hline $\mathrm{HCO} 3-$ & 0.00183 & 0.00183 & 112 & & \\
\hline $\mathrm{K}+$ & $8.10 \mathrm{e}-005$ & $8.10 \mathrm{e}-\odot \odot 5$ & 3.17 & & \\
\hline $\mathrm{Mg}++$ & $8.62 e-005$ & $8.62 \mathrm{e}-005$ & 2.10 & & \\
\hline Mo04 - - & $1.27 \mathrm{e}-006$ & 1. 27e- -006 & $\odot .203$ & & \\
\hline $\mathrm{NH} 3(\mathrm{aq})$ & $\odot .0 \odot \odot 163$ & $\odot .000163$ & 2.78 & & \\
\hline $\mathrm{Na}+$ & $\odot .000800$ & $\odot .000800$ & 18.4 & & \\
\hline $02(\mathrm{aq})$ & 0.000573 & 0.000573 & 18.3 & & \\
\hline Re04 - & $3.26 e-006$ & $3.26 e-006$ & 0.814 & & \\
\hline SO4 - - & ๑. .0००102 & ๑. .000102 & 9.79 & & \\
\hline Si02(aq) & ๑. . 000389 & $\odot .000389$ & 23.4 & & \\
\hline
\end{tabular}




\begin{tabular}{|c|c|c|c|c|c|}
\hline \multirow{2}{*}{\multicolumn{2}{|c|}{$\begin{array}{l}\text { Elemental composition } \\
\text { total moles }\end{array}$}} & \multicolumn{2}{|c|}{ In fluid } & \multicolumn{2}{|c|}{ Sorbed } \\
\hline & & moles & $\mathrm{mg} / \mathrm{kg}$ & moles & $\mathrm{mg} / \mathrm{kg}$ \\
\hline - - - - - - - - - & - - - - - - - - - - & - - - - - - - - - & 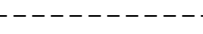 & & \\
\hline Barium & $7.811 \mathrm{e}-\odot \odot 8$ & $7.811 \mathrm{e}-\odot \odot 8$ & ๑. 01072 & & \\
\hline Calcium & 0.0002455 & $\odot .0002455$ & 9.839 & & \\
\hline Carbon & ๑. . 001833 & ๑. . 001833 & 22.01 & & \\
\hline Chlorine & $2.804 \mathrm{e}-005$ & $2.804 \mathrm{e}-005$ & 0.9937 & & \\
\hline Fluorine & $3.025 e-005$ & $3.025 e-005$ & 0.5746 & & \\
\hline Hydrogen & 111.0 & 111.0 & $1.119 \mathrm{e}+005$ & & \\
\hline Magnesium & $8.624 \mathrm{e}-005$ & $8.624 \mathrm{e}-005$ & 2.096 & & \\
\hline Molybdenum & $1.272 \mathrm{e}-006$ & $1.272 \mathrm{e}-006$ & $\odot .1220$ & & \\
\hline Nitrogen & ๑. .๑०1635 & $\odot .0 \odot \odot 1635$ & 2.289 & & \\
\hline oxygen & 55.52 & 55.52 & $8.880 e+005$ & & \\
\hline Potassium & $8.105 \mathrm{e}-005$ & $8.105 \mathrm{e}-005$ & 3.168 & & \\
\hline Rhenium & $3.256 \mathrm{e}-006$ & $3.256 \mathrm{e}-006$ & 0.6061 & & \\
\hline Silicon & ๑. $๑ \odot \odot 3889$ & ๑. $\odot \odot \odot 3889$ & 10.92 & & \\
\hline Sodium & $\odot .0008005$ & $\odot .0008005$ & 18.40 & & \\
\hline Sulfur & 0.0001019 & 0.0001019 & 3.268 & & \\
\hline
\end{tabular}




\section{Sample D1119MMB_ts}

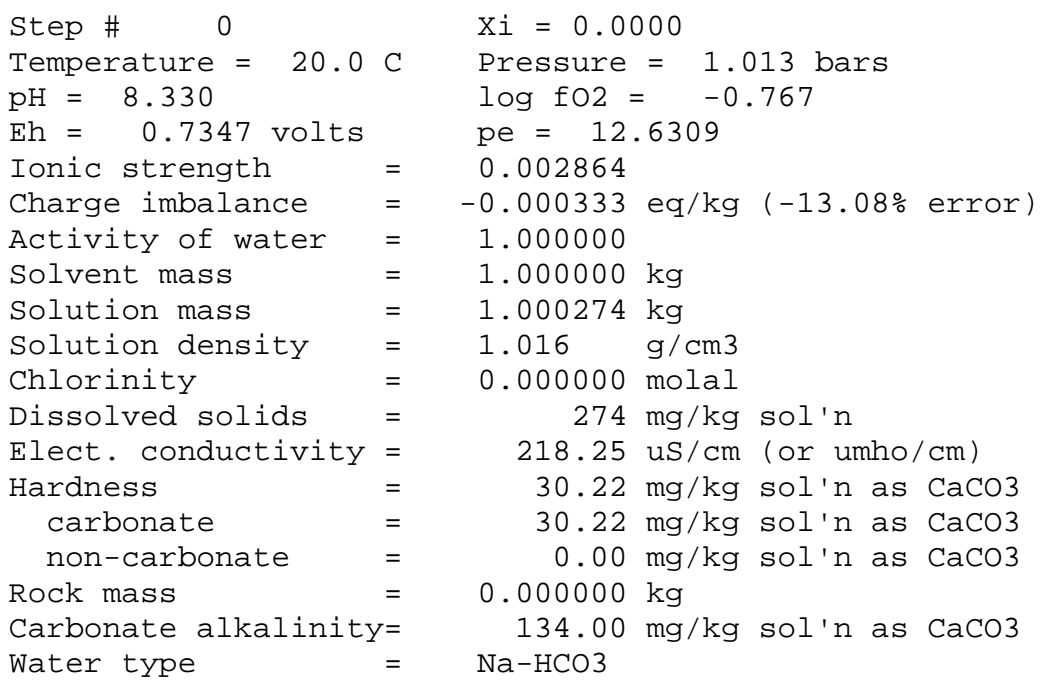

No minerals in system.

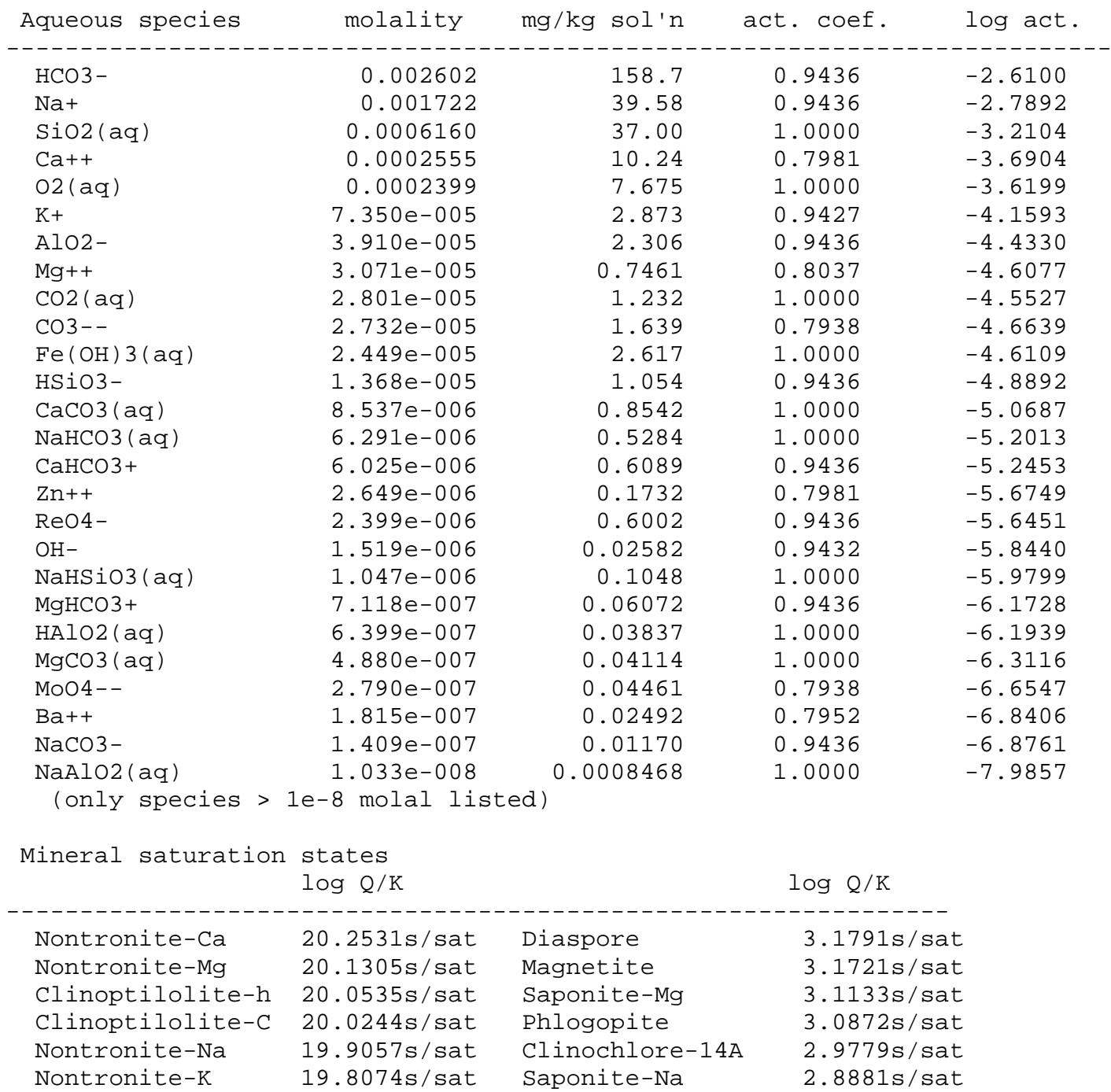




\begin{tabular}{|c|c|c|c|}
\hline Nontronite-H & $19.0943 \mathrm{~s} / \mathrm{sat}$ & Wairakite & $2.8069 \mathrm{~s} / \mathrm{sat}$ \\
\hline Clinoptilolite-h & $17.1140 \mathrm{~s} / \mathrm{sat}$ & Saponite-K & $2.7897 \mathrm{~s} / \mathrm{sat}$ \\
\hline Clinoptilolite-N & $17.1132 \mathrm{~s} / \mathrm{sat}$ & Boehmite & $2.7667 \mathrm{~s} / \mathrm{sat}$ \\
\hline Clinoptilolite-K & $16.3755 \mathrm{~s} / \mathrm{sat}$ & Gibbsite & $2.6072 \mathrm{~s} / \mathrm{sat}$ \\
\hline Clinoptilolite-h & 16.2656s/sat & Albite_high & $2.4073 \mathrm{~s} / \mathrm{sat}$ \\
\hline Hematite & $14.2866 \mathrm{~s} / \mathrm{sat}$ & Corundum & $2.2593 \mathrm{~s} / \mathrm{sat}$ \\
\hline Stilbite & $14.2794 \mathrm{~s} / \mathrm{sat}$ & Saponite-H & $2.0767 \mathrm{~s} / \mathrm{sat}$ \\
\hline Ferrite-Zn & $13.3508 \mathrm{~s} / \mathrm{sat}$ & Witherite & $1.9303 \mathrm{~s} / \mathrm{sat}$ \\
\hline Muscovite & $12.3371 \mathrm{~s} / \mathrm{sat}$ & Talc & $1.8563 \mathrm{~s} / \mathrm{sat}$ \\
\hline Epidote-ord & $11.0439 \mathrm{~s} / \mathrm{sat}$ & Kyanite & $1.7960 \mathrm{~s} / \mathrm{sat}$ \\
\hline Epidote & $11.0435 \mathrm{~s} / \mathrm{sat}$ & Andalusite & $1.5125 \mathrm{~s} / \mathrm{sat}$ \\
\hline Andradite & 9.9102s/sat & Jadeite & $1.2142 \mathrm{~s} / \mathrm{sat}$ \\
\hline Mesolite & $9.7772 \mathrm{~s} / \mathrm{sat}$ & Sillimanite & $1.1390 \mathrm{~s} / \mathrm{sat}$ \\
\hline Paragonite & $9.6723 \mathrm{~s} / \mathrm{sat}$ & Smectite-high-Fe & $1.0019 \mathrm{~s} / \mathrm{sat}$ \\
\hline Herschelite & $9.6618 \mathrm{~s} / \mathrm{sat}$ & Quartz & $0.9214 \mathrm{~s} / \mathrm{sat}$ \\
\hline Illite & $9.3967 \mathrm{~s} / \mathrm{sat}$ & Tridymite & $0.7238 \mathrm{~s} / \mathrm{sat}$ \\
\hline Beidellite-Ca & $9.2635 \mathrm{~s} / \mathrm{sat}$ & Dawsonite & $0.6999 \mathrm{~s} / \mathrm{sat}$ \\
\hline Beidellite-Mg & $9.1408 \mathrm{~s} / \mathrm{sat}$ & Anorthite & $0.4638 \mathrm{~s} / \mathrm{sat}$ \\
\hline Beidellite-Na & $8.9160 \mathrm{~s} / \mathrm{sat}$ & Dolomite & $0.4565 \mathrm{~s} / \mathrm{sat}$ \\
\hline Beidellite-K & $8.8178 \mathrm{~s} / \mathrm{sat}$ & Dolomite-ord & $0.4565 \mathrm{~s} / \mathrm{sat}$ \\
\hline Montmor-Ca & $8.4321 \mathrm{~s} / \mathrm{sat}$ & Kalsilite & $0.4398 \mathrm{~s} / \mathrm{sat}$ \\
\hline Montmor-Mg & $8.3809 \mathrm{~s} / \mathrm{sat}$ & Analcime-K & $0.3981 \mathrm{~s} / \mathrm{sat}$ \\
\hline Scolecite & $8.2829 \mathrm{~s} / \mathrm{sat}$ & Cristobalite(alp & $\odot .3594 \mathrm{~s} / \mathrm{sat}$ \\
\hline Montmor-Na & $8.1526 \mathrm{~s} / \mathrm{sat}$ & Chalcedony & $\odot .3455 \mathrm{~s} / \mathrm{sat}$ \\
\hline Beidellite-H & $8.1048 \mathrm{~s} / \mathrm{sat}$ & Calcite & $0.1073 \mathrm{~s} / \mathrm{sat}$ \\
\hline Montmor-K & $8.0583 \mathrm{~s} / \mathrm{sat}$ & Coesite & $0.0982 \mathrm{~s} / \mathrm{sat}$ \\
\hline Pyrophyllite & $7.8874 \mathrm{~s} / \mathrm{sat}$ & Aragonite & -0.0372 \\
\hline Kaolinite & $7.7209 \mathrm{~s} / \mathrm{sat}$ & Cristobalite(bet & -0.0986 \\
\hline Laumontite & $7.3774 \mathrm{~s} / \mathrm{sat}$ & Ice & -0.1194 \\
\hline Amesite-14A & $6.6724 \mathrm{~s} / \mathrm{sat}$ & Analcime & -0.2866 \\
\hline Goethite & $6.6688 \mathrm{~s} / \mathrm{sat}$ & Si02 (am) & -0.4067 \\
\hline Margarite & $6.6593 \mathrm{~s} / \mathrm{sat}$ & Clinochlore-7A & -0.4370 \\
\hline Celadonite & $6.5012 \mathrm{~s} / \mathrm{sat}$ & Zincite & -0.4933 \\
\hline Maximum_Microcli & $5.5149 \mathrm{~s} / \mathrm{sat}$ & Smithsonite & -0.5057 \\
\hline K-Feldspar & $5.5137 \mathrm{~s} / \mathrm{sat}$ & Monohydrocalcite & -0.7134 \\
\hline Ferrite-Ca & $5.4306 \mathrm{~s} / \mathrm{sat}$ & Cordierite_hydr & -0.7597 \\
\hline Lawsonite & $5.0048 \mathrm{~s} / \mathrm{sat}$ & $\mathrm{Zn}(\mathrm{OH}) 2($ gamma $)$ & -0.8981 \\
\hline Mordenite & $4.9536 \mathrm{~s} / \mathrm{sat}$ & $\mathrm{Zn}(\mathrm{OH}) 2$ (epsilon) & -0.9234 \\
\hline Ferrite-Mg & $4.9357 \mathrm{~s} / \mathrm{sat}$ & Dolomite-dis & -1.1253 \\
\hline Zn2SiO4 & $4.5448 \mathrm{~s} / \mathrm{sat}$ & Nepheline & -1.1734 \\
\hline Sanidine_high & $4.2818 \mathrm{~s} / \mathrm{sat}$ & $\mathrm{Zn}(\mathrm{OH}) 2($ beta $)$ & -1.1993 \\
\hline Prehnite & $3.8754 \mathrm{~s} / \mathrm{sat}$ & Magnesite & -1.3109 \\
\hline Natrolite & $3.8066 \mathrm{~s} / \mathrm{sat}$ & Tremolite & -1.6943 \\
\hline Clinozoisite & $3.7805 \mathrm{~s} / \mathrm{sat}$ & Chrysotile & -1.9870 \\
\hline Albite_low & $3.7596 \mathrm{~s} / \mathrm{sat}$ & $\mathrm{Fe}(\mathrm{OH}) 3$ & -2.0191 \\
\hline Albite & $3.7595 \mathrm{~s} / \mathrm{sat}$ & Grossular & -2.5130 \\
\hline Smectite-low-Fe- & $3.7594 \mathrm{~s} / \mathrm{sat}$ & Enstatite & -2.7037 \\
\hline Zoisite & $3.7346 \mathrm{~s} / \mathrm{sat}$ & Diopside & -2.7056 \\
\hline Saponite-Ca & $3.2354 \mathrm{~s} / \mathrm{sat}$ & & \\
\hline (only minerals & ith $\log Q / K>$ & 3 listed) & \\
\hline Gases & fugacity & $\log$ fug. & \\
\hline$-\ldots-\ldots$ & - - - - - - - - & ----- & \\
\hline $02(g)$ & 0.1711 & -0.767 & \\
\hline $\mathrm{H} 20$ (g) & ๑. . 01903 & -1.721 & \\
\hline $\operatorname{co2}(\mathrm{g})$ & 0.0007004 & -3.155 & \\
\hline $\mathrm{H} 2(\mathrm{~g})$ & $9.059 \mathrm{e}-043$ & -42.043 & \\
\hline $\operatorname{co}(g)$ & $2.027 e-049$ & -48.693 & \\
\hline $\mathrm{Zn}(\mathrm{g})$ & $4.928 e-075$ & -74.307 & \\
\hline $\mathrm{Na}(\mathrm{g})$ & $1.317 \mathrm{e}-076$ & -75.880 & \\
\hline K(g) & $1.127 \mathrm{e}-078$ & -77.948 & \\
\hline $\operatorname{Mg}(g)$ & $8.653 e-132$ & -131.063 & \\
\hline $\mathrm{CH} 4(\mathrm{~g})$ & $2.235 e-148$ & -147.651 & \\
\hline
\end{tabular}




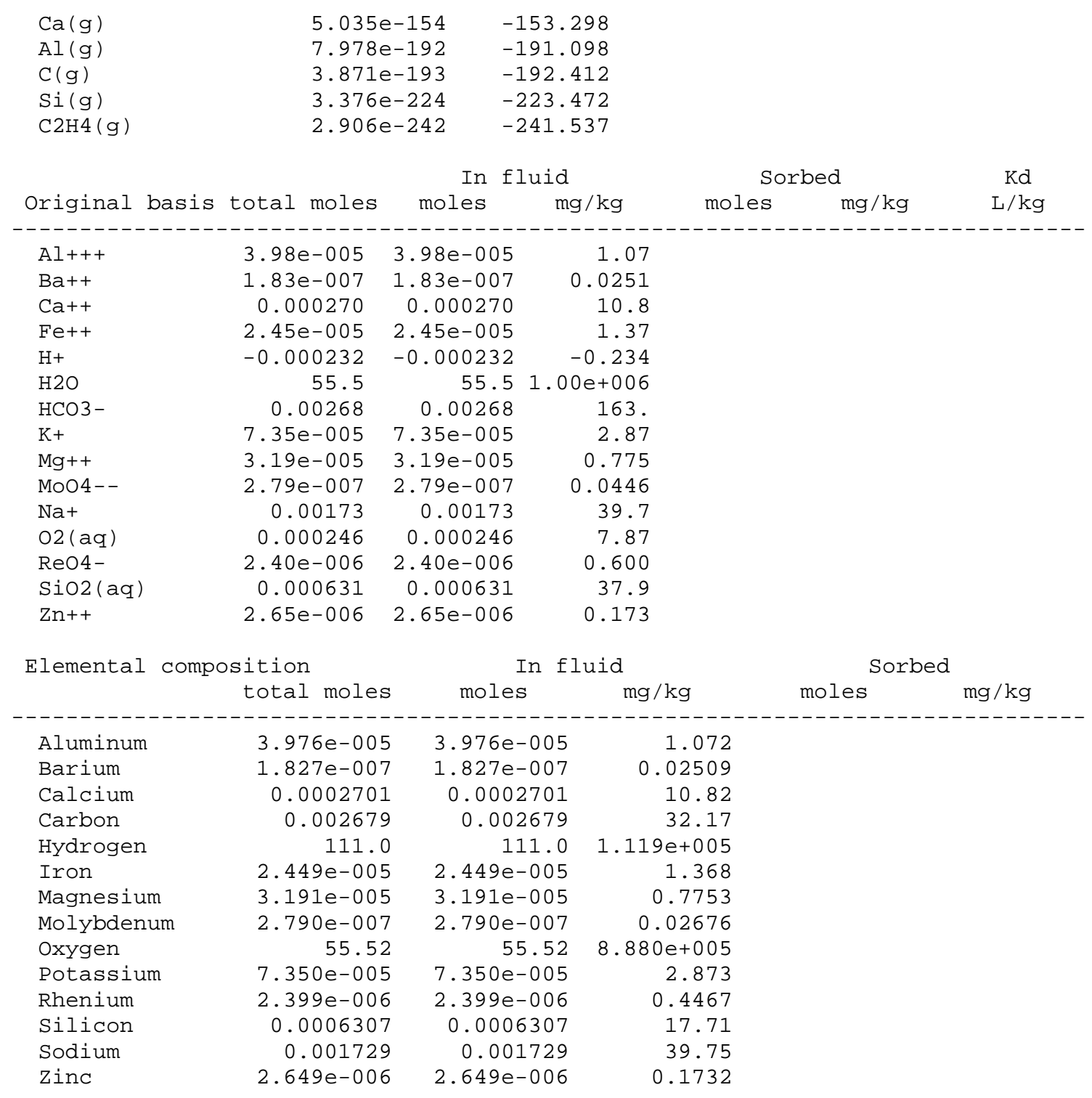




\section{Sample D1119MMB_bs}

$\begin{array}{ll}\text { Step \# } \odot & \\ \text { Temperature }=20 . \odot \mathrm{C} \\ \text { pH }=8.420 \\ \text { Eh }=0.7294 \text { volts } \\ \text { Ionic strength } & = \\ \text { Charge imbalance } & = \\ \text { Activity of water } & = \\ \text { Solvent mass } & = \\ \text { Solution mass } & = \\ \text { Solution density } & = \\ \text { Chlorinity } & = \\ \text { Dissolved solids } & = \\ \text { Elect. conductivity } & = \\ \text { Hardness } & = \\ \text { carbonate } & = \\ \text { non-carbonate } & = \\ \text { Rock mass } & = \\ \text { Carbonate alkalinity= } & = \\ \text { Water type } & =\end{array}$

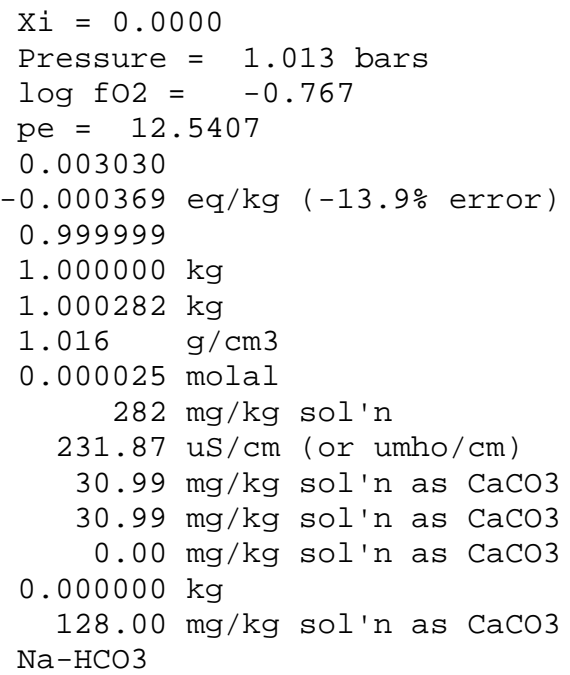

No minerals in system.

\begin{tabular}{|c|c|c|c|c|}
\hline Aqueous species & molality & $\mathrm{mg} / \mathrm{kg}$ sol'n & act. coef. & $\log$ act \\
\hline - & ----- & 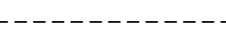 & $---{ }_{-}$ & \\
\hline $\mathrm{HCO}-$ & $\odot .002471$ & 150.7 & $\odot .9422$ & -2.6331 \\
\hline $\mathrm{Na}+$ & 0.001803 & 41.44 & $\odot .9422$ & -2.7699 \\
\hline Si02(aq) & $\odot .0006331$ & 38.03 & 1.0000 & -3.1986 \\
\hline $\mathrm{Ca}++$ & 0.0002605 & 10.44 & 0.7935 & -3.6847 \\
\hline $02(\mathrm{aq})$ & $\odot .0 \odot \odot 2397$ & 7.666 & 1.0000 & -3.6204 \\
\hline $\mathrm{K}+$ & $6.920 \mathrm{e}-005$ & 2.705 & $\odot .9412$ & -4.1862 \\
\hline NO3- & $6.269 e-005$ & 3.886 & 0.9412 & -4.2291 \\
\hline SO4-- & $5.144 \mathrm{e}-005$ & 4.940 & 0.7873 & -4.3926 \\
\hline Al02- & $4.102 \mathrm{e}-005$ & 2.419 & 0.9422 & -4.4128 \\
\hline $\mathrm{F}-$ & $4.096 \mathrm{e}-\odot \odot 5$ & 0.7779 & 0.9417 & -4.4138 \\
\hline CO3-- & $3.206 e-005$ & 1.924 & 0.7889 & -4.5970 \\
\hline $\mathrm{Mg}++$ & $3.07 \odot e-\odot \odot 5$ & 0.7460 & $\odot .7993$ & -4.6101 \\
\hline $\mathrm{Fe}(\mathrm{OH}) 3(\mathrm{aq})$ & $2.555 \mathrm{e}-005$ & 2.730 & 1.0000 & -4.5926 \\
\hline $\mathrm{Cl}-\mathrm{C}$ & $2.489 e-005$ & 0.8822 & 0.9412 & -4.6303 \\
\hline $\mathrm{co2}(\mathrm{aq})$ & $2.159 \mathrm{e}-0 \odot 5$ & 0.9498 & $1.00 \odot \odot$ & -4.6658 \\
\hline HSiO3- & $1.732 \mathrm{e}-005$ & 1.335 & $\odot .9422$ & -4.7874 \\
\hline CaC03(aq) & $1.009 e-005$ & 1.010 & 1.0000 & -4.9960 \\
\hline Re04- & $7.928 e-0 \odot 6$ & 1.983 & 0.9422 & -5.1267 \\
\hline $\mathrm{NaHCO} 3(\mathrm{aq})$ & $6.237 e-006$ & 0.5238 & 1.0000 & -5.2050 \\
\hline $\mathrm{CaHCO}+$ & $5.798 e-0 \odot 6$ & $\odot .5860$ & $\odot .9422$ & -5.2626 \\
\hline $\mathrm{Zn++}$ & $2.977 \mathrm{e}-006$ & 0.1946 & 0.7935 & -5.6266 \\
\hline $\mathrm{OH}-$ & $1.871 \mathrm{e}-006$ & ๑.03181 & 0.9417 & -5.7540 \\
\hline $\operatorname{NaHSiO3}(\mathrm{aq})$ & $1.384 \mathrm{e}-006$ & 0.1385 & 1.0000 & -5.8588 \\
\hline $\mathrm{CaSO} 4(\mathrm{aq})$ & $1.058 e-006$ & 0.1440 & 1.0000 & -5.9755 \\
\hline Mo04 - - & $7.324 \mathrm{e}-0 \odot 7$ & 0.1171 & $\odot .7889$ & -6.2382 \\
\hline MgHCO3+ & $6.723 e-0 \odot 7$ & 0.05734 & 0.9422 & -6.1983 \\
\hline $\operatorname{MgC0} 3(\mathrm{aq})$ & $5.661 \mathrm{e}-007$ & 0.04772 & 1.0000 & -6.2471 \\
\hline HAl02 ( aq) & $5.448 e-0 \odot 7$ & 0.03267 & $1.0 \odot \odot \odot$ & -6.2638 \\
\hline $\operatorname{MgSO} 4(\mathrm{aq})$ & $2.263 e-\odot \odot 7$ & $\odot .02723$ & 1.0000 & -6.6454 \\
\hline $\mathrm{Ba}++$ & $1.891 \mathrm{e}-007$ & ๑. 02596 & 0.7905 & -6.8254 \\
\hline NaC03 - & $1.722 \mathrm{e}-0 \odot 7$ & 0.01429 & 0.9422 & -6.7899 \\
\hline $\mathrm{CaF}+$ & $4.0 \odot 6 e-0 \odot 8$ & ๑. . 002366 & 0.9422 & -7.4231 \\
\hline $\mathrm{MgF}+$ & $2.296 e-008$ & $\odot .0009941$ & $\odot .9422$ & -7.6648 \\
\hline KSO4 - & $2.127 \mathrm{e}-\odot \odot 8$ & ๑. ๑๑2875 & $\odot .9422$ & -7.6980 \\
\hline ZnSO4(aq) & $1.771 \mathrm{e}-0 \odot 8$ & 0.002858 & $1.00 \odot \odot$ & -7.7518 \\
\hline $\operatorname{NaAl02}(\mathrm{aq})$ & $1.132 \mathrm{e}-008$ & ๑.0009273 & 1.0000 & -7.9463 \\
\hline
\end{tabular}




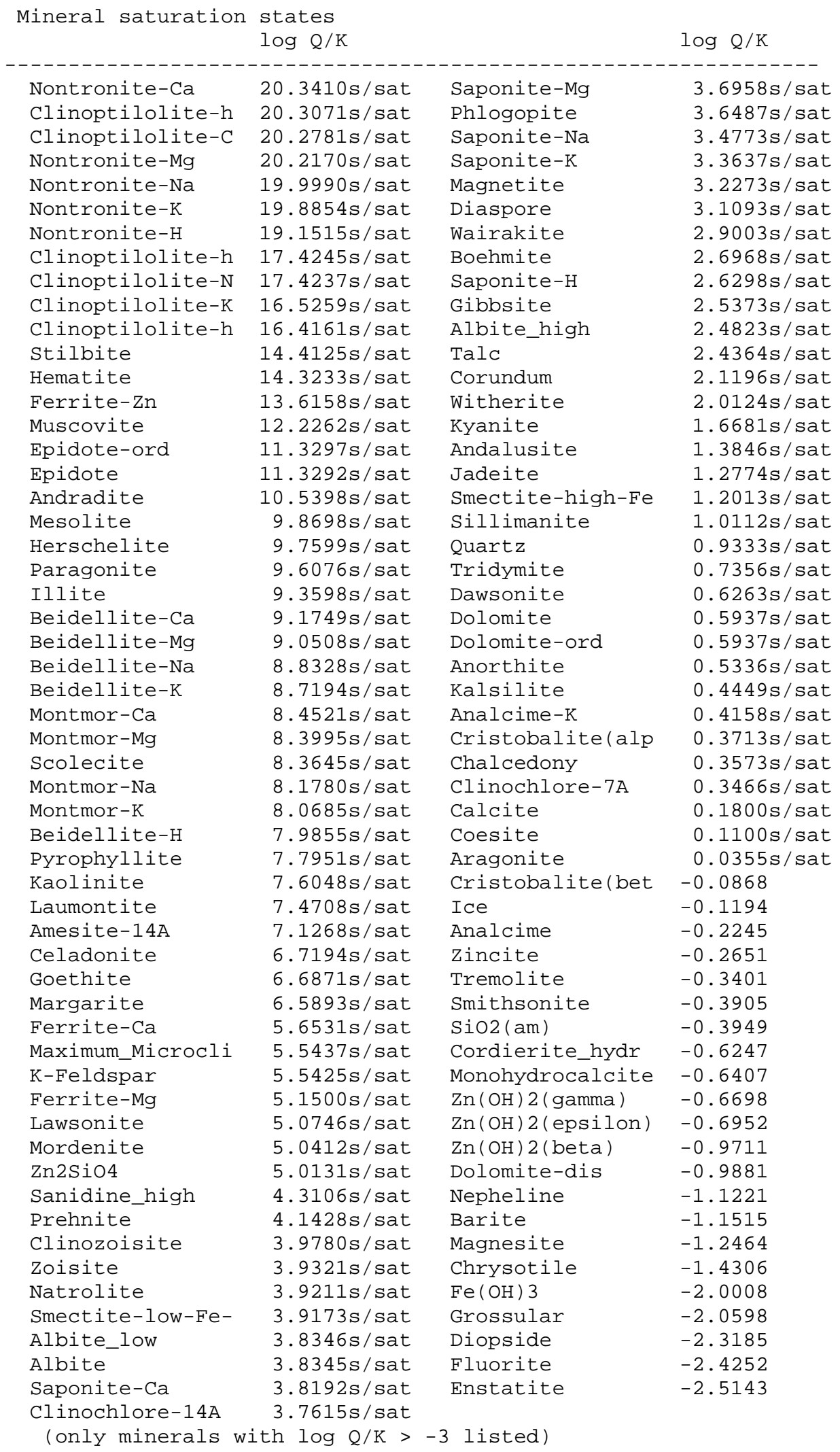




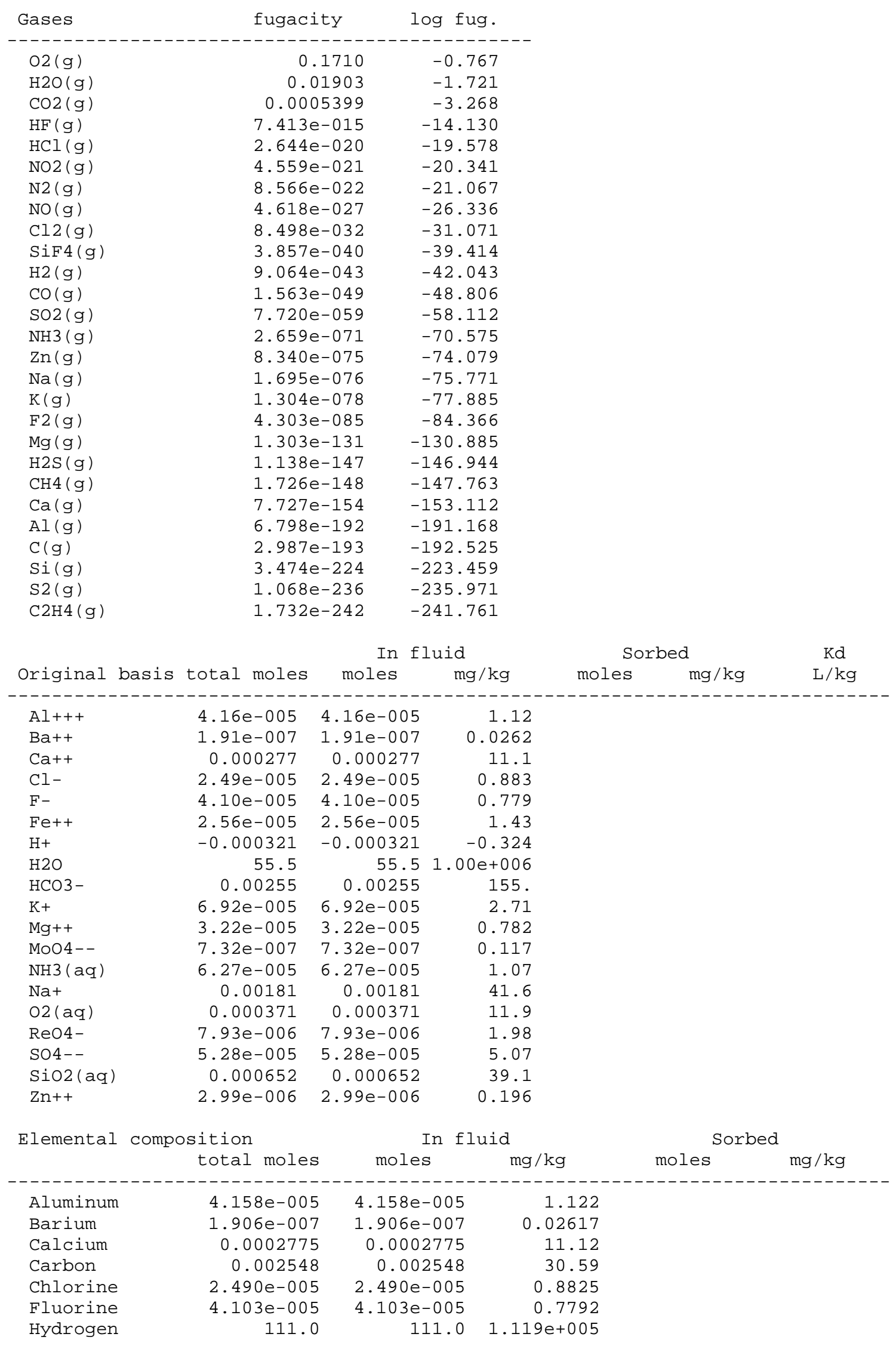




$\begin{array}{lrrr}\text { Iron } & 2.555 \mathrm{e}-005 & 2.555 \mathrm{e}-005 & 1.427 \\ \text { Magnesium } & 3.219 \mathrm{e}-005 & 3.219 \mathrm{e}-005 & 0.7822 \\ \text { Molybdenum } & 7.324 \mathrm{e}-007 & 7.324 \mathrm{e}-007 & 0.07025 \\ \text { Nitrogen } & 6.269 \mathrm{e}-005 & 6.269 \mathrm{e}-005 & 0.8779 \\ \text { Oxygen } & 55.52 & 55.52 & 8.880 \mathrm{e}+005 \\ \text { Potassium } & 6.922 \mathrm{e}-005 & 6.922 \mathrm{e}-005 & 2.706 \\ \text { Rhenium } & 7.928 \mathrm{e}-006 & 7.928 \mathrm{e}-006 & 1.476 \\ \text { Silicon } & 0.0006518 & 0.0006518 & 18.30 \\ \text { Sodium } & 0.001811 & 0.001811 & 41.62 \\ \text { Sulfur } & 5.276 \mathrm{e}-005 & 5.276 \mathrm{e}-005 & 1.691 \\ \text { Zinc } & 2.995 \mathrm{e}-006 & 2.995 \mathrm{e}-006 & 0.1958\end{array}$




\section{Sample D11NEND}

$\begin{array}{ll}\text { Step \# } \odot & \\ \text { Temperature }=20 . \odot \mathrm{C} \\ \text { pH }=8.140 \\ \text { Eh = } \odot .7459 \text { volts } \\ \text { Ionic strength } \\ \text { Charge imbalance }= \\ \text { Activity of water }= \\ \text { Solvent mass } & = \\ \text { Solution mass } & = \\ \text { Solution density } & = \\ \text { Chlorinity } & = \\ \text { Dissolved solids } & = \\ \text { Elect. conductivity } & = \\ \text { Hardness } & = \\ \text { carbonate } & = \\ \text { non-carbonate } & = \\ \text { Rock mass } & = \\ \text { Carbonate alkalinity } & = \\ \text { Water type } & =\end{array}$

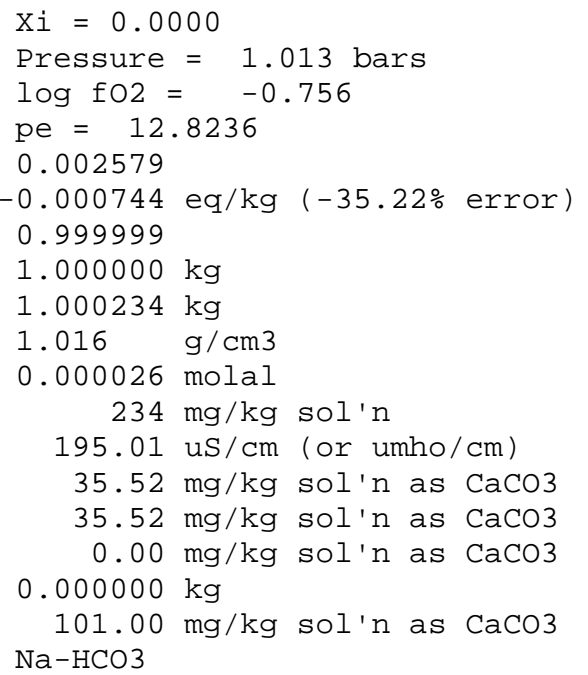

No minerals in system.

\begin{tabular}{|c|c|c|c|c|}
\hline Aqueous species & molality & /kg sol'n & act. coef. & $\log$ act. \\
\hline - - - - - - - - - - - - & ------- & ------ & ------ & \\
\hline HCO3 - & $\odot .0 \odot 1979$ & 120.7 & $\odot .9462$ & -2.7277 \\
\hline $\mathrm{Na}+$ & $\odot .0009686$ & 22.26 & $\odot .9462$ & -3.0378 \\
\hline Si02(aq) & $\odot .0 \odot \odot 5213$ & 31.32 & 1.0000 & -3.2829 \\
\hline $02(\mathrm{aq})$ & $\odot .0002460$ & 7.871 & 1.0000 & -3.6090 \\
\hline $\mathrm{Ca}++$ & $\odot .0 \odot \odot 2446$ & 9.800 & 0.8065 & -3.7050 \\
\hline N03- & $\odot .0001619$ & 10.04 & $\odot .9454$ & -3.8152 \\
\hline S04-- & $\odot .0001129$ & 10.84 & $\odot .8011$ & -4.0437 \\
\hline $\mathrm{Mg}++$ & $9.548 \mathrm{e}-005$ & 2.320 & 0.8116 & -4.1107 \\
\hline $\mathrm{K}+$ & $8.476 e-005$ & 3.313 & 0.9454 & -4.0962 \\
\hline $\mathrm{F}-$ & $4.117 e-\odot \odot 5$ & $\odot .7820$ & $\odot .9458$ & -4.4096 \\
\hline $\mathrm{co2}(\mathrm{aq})$ & $3.308 e-005$ & 1.456 & 1.0000 & -4.4804 \\
\hline $\mathrm{Cl}-$ & $2.595 \mathrm{e}-\odot \odot 5$ & $\odot .9197$ & $\odot .9454$ & -4.6103 \\
\hline CO3-- & $1.330 \mathrm{e}-005$ & $\odot .7982$ & 0.8025 & -4.9716 \\
\hline Re04 - & $1.311 \mathrm{e}-005$ & 3.279 & $\odot .9462$ & -4.9065 \\
\hline HSi03- & $7.452 \mathrm{e}-\odot \odot 6$ & 0.5744 & $\odot .9462$ & -5.1517 \\
\hline CaHCO3+ & $4.431 e-006$ & $\odot .4479$ & $\odot .9462$ & -5.3775 \\
\hline $\mathrm{CaC03}(\mathrm{aq})$ & $4.065 e-\odot \odot 6$ & 0.4068 & 1.0000 & -5.3909 \\
\hline $\mathrm{NaHCO} 3(\mathrm{aq})$ & $2.706 \mathrm{e}-\odot \odot 6$ & 0.2273 & 1.0000 & -5.5676 \\
\hline CaS04 $(\mathrm{aq})$ & $2.255 e-\odot \odot 6$ & $\odot .3069$ & 1.0000 & -5.6469 \\
\hline $\mathrm{MgHCO}+$ & $1.700 \mathrm{e}-\odot \odot 6$ & 0.1450 & $\odot .9462$ & -5.7936 \\
\hline $\operatorname{MgS0} 4(\mathrm{aq})$ & $1.595 e-006$ & 0.1920 & 1.0000 & -5.7972 \\
\hline Mo04 - - & $1.169 \mathrm{e}-\odot \odot 6$ & 0.1870 & $\odot .8025$ & -6.0276 \\
\hline $\mathrm{OH}-$ & $9.777 \mathrm{e}-\odot \odot 7$ & ๑. .01662 & $\odot .9458$ & -6.0340 \\
\hline $\operatorname{MgC03}(\mathrm{aq})$ & $7.545 e-\odot \odot 7$ & 0.06360 & 1.0000 & -6.1223 \\
\hline NaHSi03(aq) & $3.228 e-0 \odot 7$ & 0.03230 & 1.0000 & -6.4911 \\
\hline $\mathrm{Ba}++$ & $9.355 e-0 \odot 8$ & 0.01284 & 0.8038 & -7.1238 \\
\hline $\mathrm{MgF}+$ & $7.290 \mathrm{e}-\odot \odot 8$ & $\odot .003156$ & $\odot .9462$ & -7.1613 \\
\hline KSO4- & $5.820 \mathrm{e}-\odot \odot 8$ & $\odot .007864$ & $\odot .9462$ & -7.2591 \\
\hline NaCO3 - & $3.904 \mathrm{e}-\odot \odot 8$ & $\odot .003239$ & 0.9462 & -7.4325 \\
\hline $\mathrm{CaF}+$ & $3.843 e-008$ & $\odot .002270$ & $\odot .9462$ & -7.4393 \\
\hline (only species > & 1e-8 molal listed) & & & \\
\hline \multirow[t]{2}{*}{ Mineral saturation } & states & & & \\
\hline & $\log Q / K$ & & $\log Q / K$ & \\
\hline \multicolumn{5}{|c|}{ 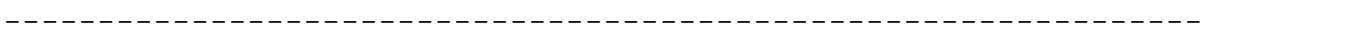 } \\
\hline Talc & $1.9172 \mathrm{~s} / \mathrm{sat} \quad \mathrm{Ca}$ & lcite & -0.2149 & \\
\hline Witherite & $1.3394 \mathrm{~s} / \mathrm{sat}$ & agonite & -0.3594 & \\
\hline
\end{tabular}




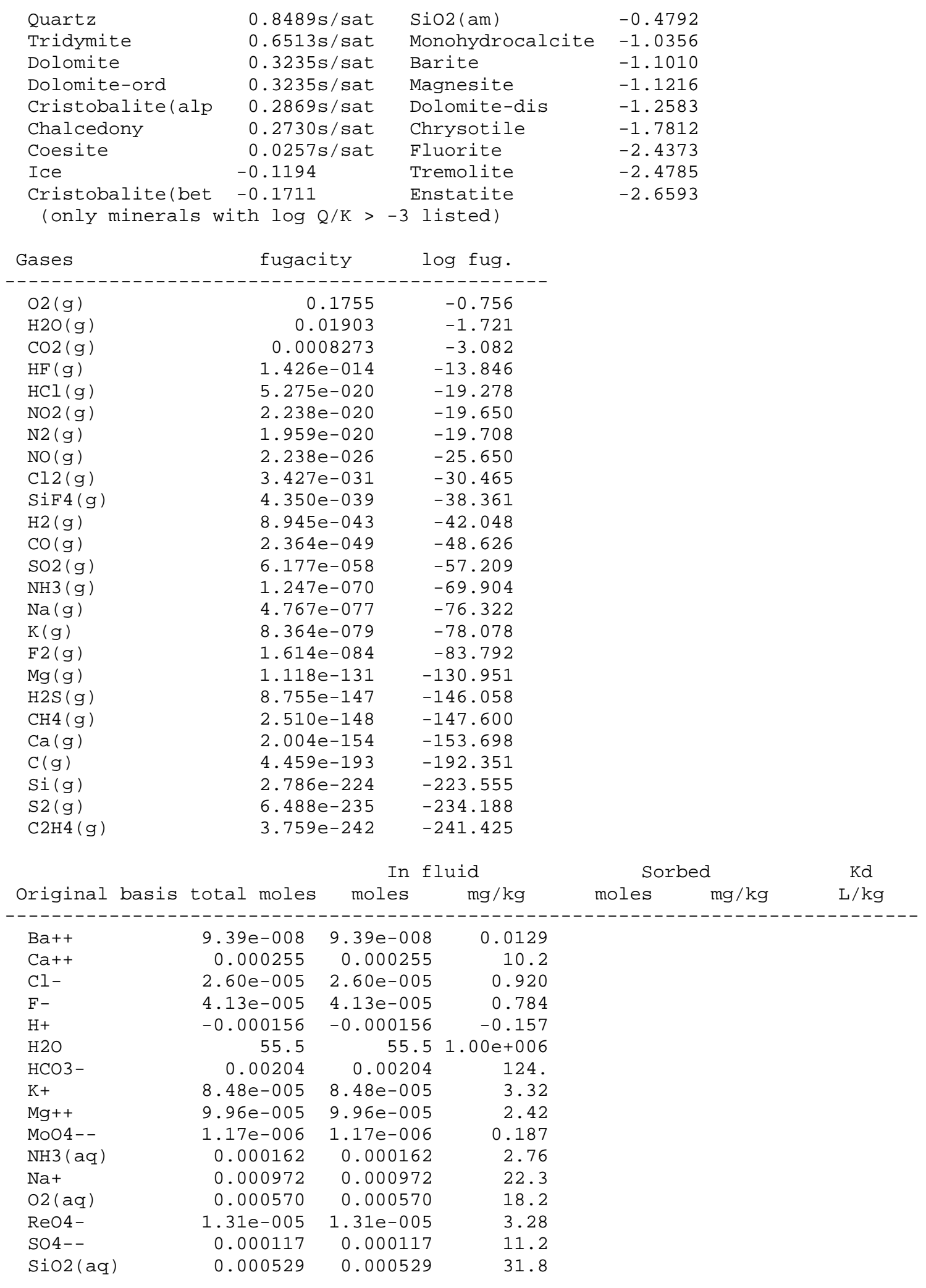




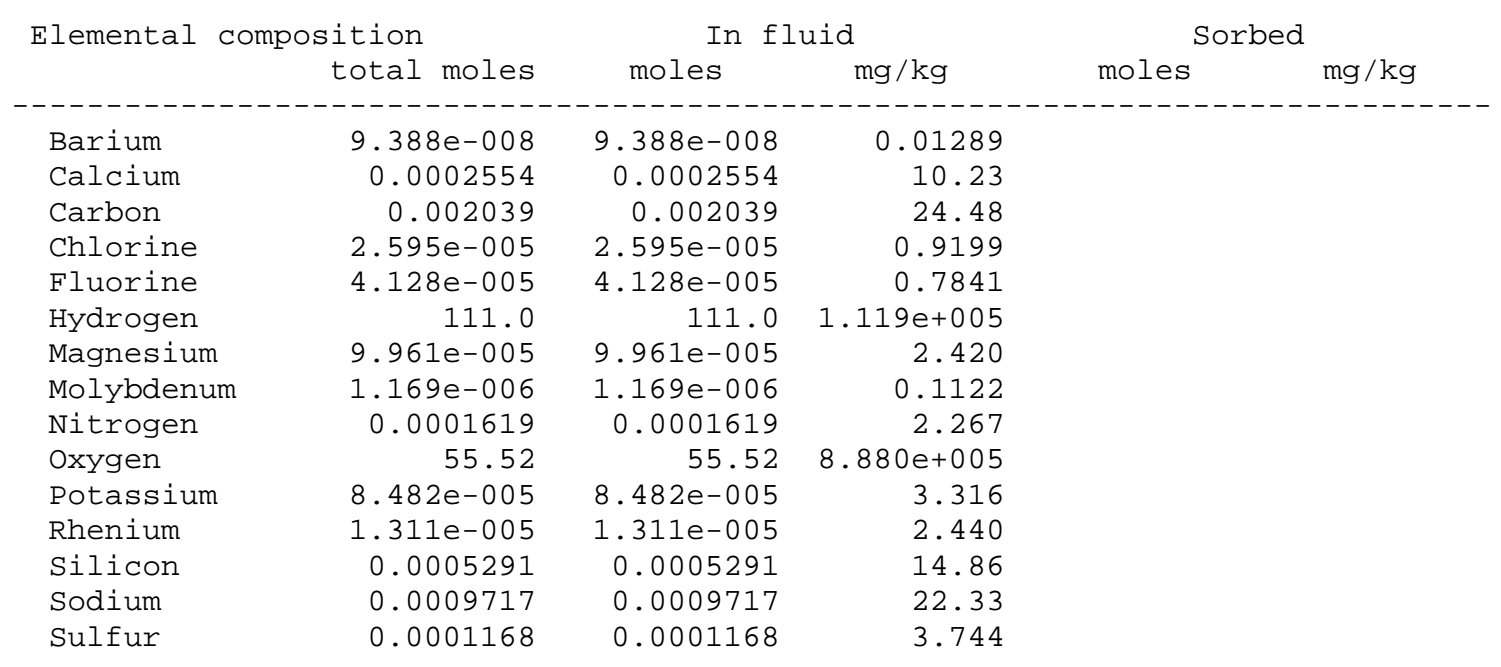




\section{Sample D14BG}

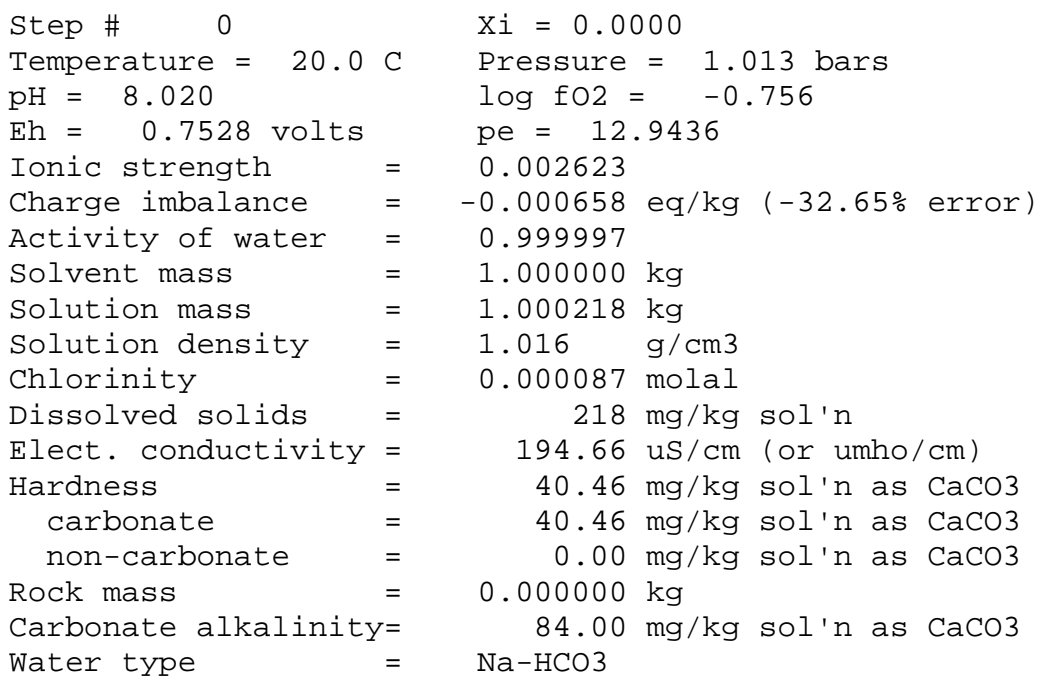

No minerals in system.

\begin{tabular}{|c|c|c|c|c|}
\hline Aqueous species & molality & $\mathrm{mg} / \mathrm{kg}$ sol'n & act. coef. & $\log$ act \\
\hline - & & & $-\ldots-\ldots$ & 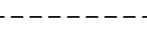 \\
\hline $\mathrm{HCO}-$ & $\odot .001651$ & 100.7 & $\odot .9458$ & -2.8065 \\
\hline $\mathrm{Na}+$ & ๑. . ๑९८411 & 19.33 & $\odot .9458$ & -3.0994 \\
\hline $\operatorname{Si02}(\mathrm{aq})$ & 0.0005163 & 31.01 & 1.0000 & -3.2871 \\
\hline $\mathrm{Ca}++$ & $\odot .0 \odot \odot 2778$ & 11.13 & 0.8052 & -3.6503 \\
\hline $02(\mathrm{aq})$ & $\odot .0 \odot \odot 2460$ & 7.871 & $1.00 \odot \odot$ & -3.6090 \\
\hline SO4 - - & ๑. . 0002110 & 20.26 & 0.7997 & -3.7728 \\
\hline N03- & $\odot .0 \odot \odot 1267$ & 7.851 & $\odot .9450$ & -3.9220 \\
\hline $\mathrm{Mg}++$ & $\odot .0 \odot \odot 1090$ & 2.648 & $\odot .8104$ & -4.0540 \\
\hline $\mathrm{Cl}-$ & $8.686 \mathrm{e}-005$ & 3.079 & 0.9450 & -4.0857 \\
\hline $\mathrm{K}+$ & $6.661 \mathrm{e}-005$ & 2.604 & $\odot .9450$ & -4.2010 \\
\hline $\mathrm{co2}(\mathrm{aq})$ & $3.637 e-005$ & 1.600 & 1.0000 & -4.4392 \\
\hline $\mathrm{F}-$ & $3.382 e-0 \odot 5$ & 0.6424 & $\odot .9454$ & -4.4952 \\
\hline CO3-- & $8.432 \mathrm{e}-\odot \odot 6$ & $\odot .5059$ & 0.8011 & -5.1704 \\
\hline HSi03 - & 5. $601 \mathrm{e}-006$ & $\odot .4317$ & $\odot .9458$ & -5.2759 \\
\hline CaSO4(aq) & $4.771 \mathrm{e}-006$ & 0.6494 & 1.0000 & -5.3214 \\
\hline $\mathrm{CaHCO} 3+$ & $4.193 e-0 \odot 6$ & 0.4238 & $\odot .9458$ & -5.4016 \\
\hline $\operatorname{MgSO} 4(\mathrm{aq})$ & $3.392 \mathrm{e}-006$ & $\odot .4081$ & 1.0000 & -5.4696 \\
\hline Cac03 (aq) & $2.917 \mathrm{e}-\odot \odot 6$ & $\odot .2919$ & $1.0 \odot \odot \odot$ & -5.5351 \\
\hline Re04 - & $2.135 \mathrm{e}-\odot \odot 6$ & $\odot .5341$ & $\odot .9458$ & -5.6948 \\
\hline $\mathrm{NaHCO} 3(\mathrm{aq})$ & 1. $959 \mathrm{e}-006$ & 0.1646 & 1.0000 & -5.7079 \\
\hline $\mathrm{MgHCO} 3+$ & 1. $616 \mathrm{e}-006$ & 0.1379 & $\odot .9458$ & -5.8157 \\
\hline $\mathrm{OH}-$ & $7.420 \mathrm{e}-0 \odot 7$ & 0.01262 & 0.9454 & -6.1540 \\
\hline $\operatorname{MgC0} 3(\mathrm{aq})$ & $5.440 \mathrm{e}-\odot \odot 7$ & ๑. 04585 & $1.00 \odot \odot$ & -6.2644 \\
\hline NaHSiO3(aq) & $2.105 \mathrm{e}-\odot \odot 7$ & 0.02106 & $1.000 \odot$ & -6.6768 \\
\hline $\mathrm{Ba}++$ & $1.037 \mathrm{e}-\odot \odot 7$ & $\odot .01424$ & 0.8025 & -7.0799 \\
\hline KSO4 - & $8.534 \mathrm{e}-\odot \odot 8$ & $\odot .01153$ & $\odot .9458$ & -7.0931 \\
\hline $\mathrm{MgF}+$ & $6.823 e-0 \odot 8$ & ๑. .००2954 & $\odot .9458$ & -7.1902 \\
\hline Mo04 - - & $5.334 e-0 \odot 8$ & ๑. . 008529 & 0.8011 & -7.3693 \\
\hline $\mathrm{CaF}+$ & $3.581 \mathrm{e}-\odot \odot 8$ & $\odot .0 \odot 2115$ & 0.9458 & -7.4702 \\
\hline NaCO3 - & $2.145 e-0 \odot 8$ & 0.001780 & 0.9458 & -7.6928 \\
\hline $\mathrm{NaCl}(\mathrm{aq})$ & 1. $065 \mathrm{e}-0 \odot 8$ & $\odot .0006220$ & 1.0000 & -7.9728 \\
\hline $\mathrm{H}+$ & 1. $. \odot 6 e-\odot \odot 8$ & 1. $013 e-\odot \odot 5$ & $\odot .9497$ & -8.0200 \\
\hline
\end{tabular}




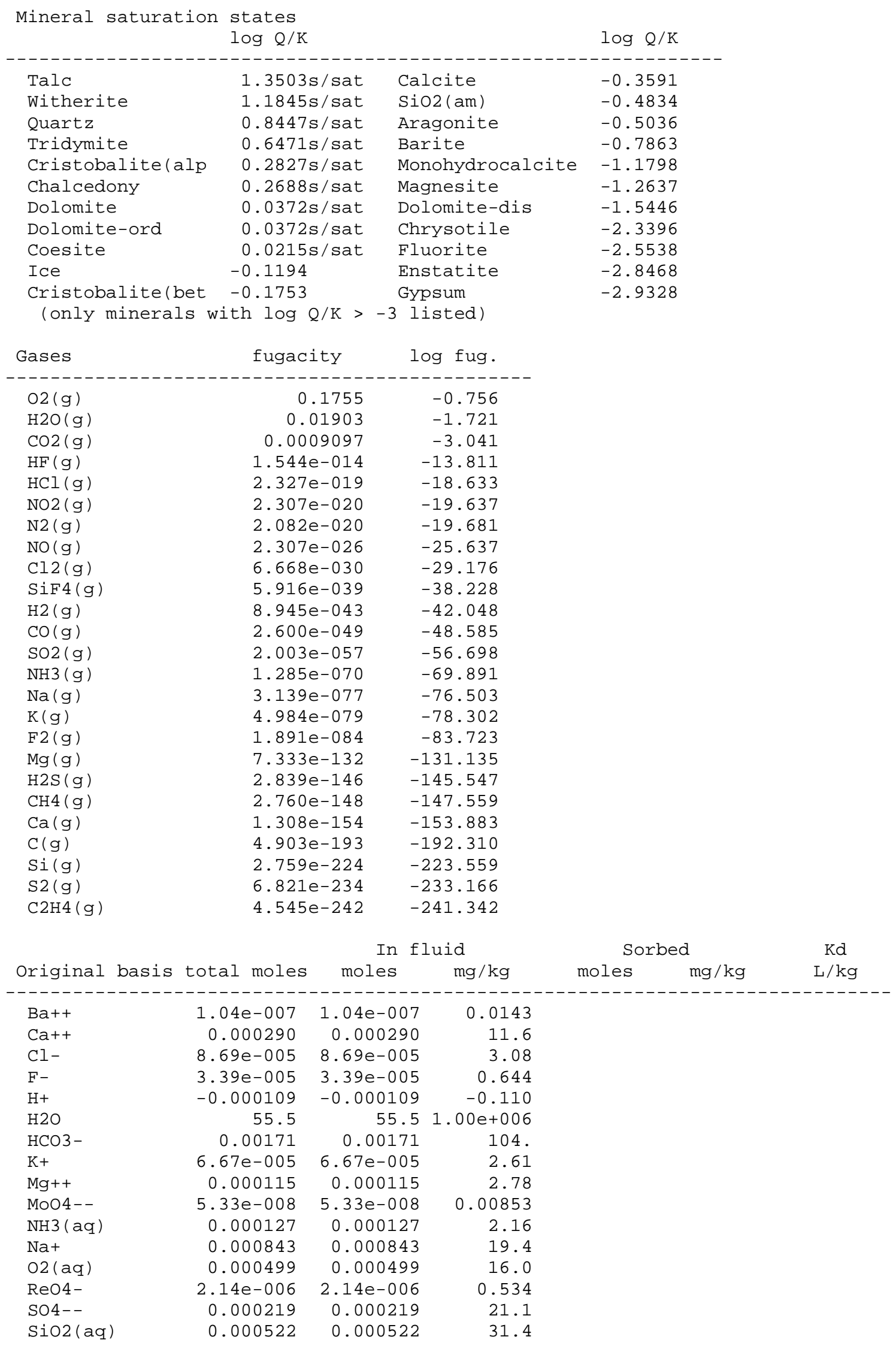




\begin{tabular}{|c|c|c|c|c|c|}
\hline \multirow{2}{*}{\multicolumn{2}{|c|}{$\begin{array}{l}\text { Elemental composition } \\
\text { total moles }\end{array}$}} & \multicolumn{2}{|c|}{ In fluid } & \multicolumn{2}{|c|}{ Sorbed } \\
\hline & & moles & $\mathrm{mg} / \mathrm{kg}$ & moles & $\mathrm{mg} / \mathrm{kg}$ \\
\hline Barium & $1.039 \mathrm{e}-0 \odot 7$ & 1. $039 \mathrm{e}-0 \odot 7$ & ๑. . 01427 & & \\
\hline Calcium & ๑. $0 \odot \odot 2897$ & ๑. $0 \odot \odot 2897$ & 11.61 & & \\
\hline Carbon & $\odot .0 \odot 17 \odot 7$ & ๑. . 001707 & $2 \odot .50$ & & \\
\hline Chlorine & $8.688 e-005$ & $8.688 e-005$ & 3.080 & & \\
\hline Fluorine & $3.393 e-0 \odot 5$ & $3.393 e-005$ & 0.6444 & & \\
\hline Hydrogen & 111.0 & 111.0 & $1.119 \mathrm{e}+005$ & & \\
\hline Magnesium & $\odot .0 \odot \odot 1146$ & $\odot .0 \odot \odot 1146$ & 2.784 & & \\
\hline Molybdenum & $5.334 \mathrm{e}-\odot \odot 8$ & $5.334 \mathrm{e}-0 \odot 8$ & $\odot .005116$ & & \\
\hline Nitrogen & $\odot .0001267$ & $\odot .0001267$ & 1.774 & & \\
\hline oxygen & 55.52 & 55.52 & $8.880 \mathrm{e}+005$ & & \\
\hline Potassium & $6.670 \mathrm{e}-0 \odot 5$ & $6.670 \mathrm{e}-\odot \odot 5$ & 2.607 & & \\
\hline Rhenium & $2.135 e-006$ & $2.135 e-006$ & 0.3975 & & \\
\hline Silicon & $\odot .0 \odot \odot 5221$ & $\odot .0 \odot \odot 5221$ & 14.66 & & \\
\hline Sodium & $\odot .0008433$ & 0.0008433 & 19.38 & & \\
\hline Sulfur & ๑. .0002192 & $\odot .0002192$ & 7.028 & & \\
\hline
\end{tabular}




\section{Sample D11SWTOP}

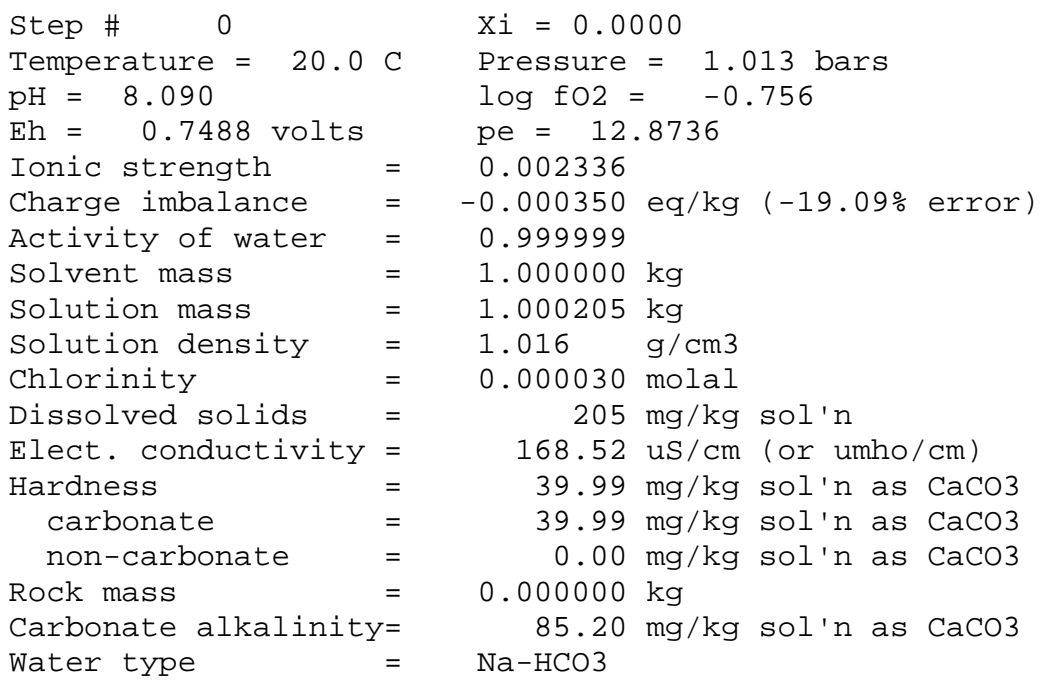

No minerals in system.

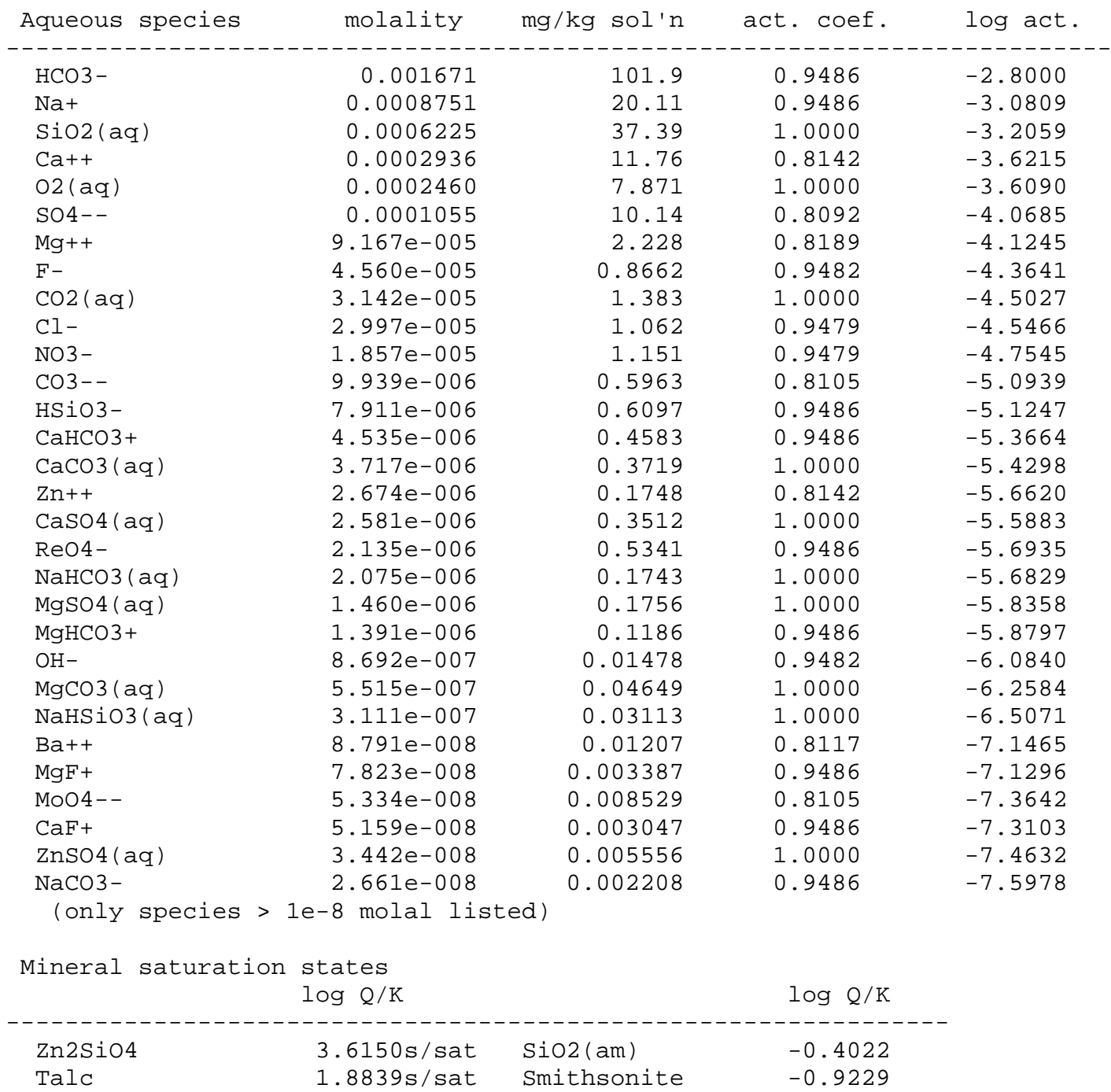




\begin{tabular}{|c|c|c|c|c|c|c|}
\hline Witherite & \multicolumn{2}{|c|}{$1.1943 \mathrm{~s} / \mathrm{sat}$} & \multicolumn{2}{|c|}{ Zincite } & \multicolumn{2}{|l|}{-0.9605} \\
\hline Quartz & \multicolumn{2}{|c|}{$0.9259 \mathrm{~s} / \mathrm{sat}$} & \multicolumn{2}{|c|}{ Monohydrocalcite } & -1.0745 & \\
\hline Tridymite & \multicolumn{2}{|c|}{$0.7283 \mathrm{~s} / \mathrm{sat}$} & \multicolumn{2}{|c|}{ Barite } & -1.1486 & \\
\hline Cristobalite(alp & \multicolumn{2}{|c|}{$0.3639 \mathrm{~s} / \mathrm{sat}$} & \multicolumn{2}{|c|}{ Magnesite } & -1.2577 & \\
\hline Chalcedony & \multicolumn{2}{|c|}{$0.3500 \mathrm{~s} / \mathrm{sat}$} & \multicolumn{2}{|c|}{$\mathrm{Zn}(\mathrm{OH}) 2($ gamma $)$} & -1.3652 & \\
\hline Dolomite & \multicolumn{2}{|c|}{$0.1485 \mathrm{~s} / \mathrm{sat}$} & $\mathrm{Zn}($ & $\mathrm{OH}) 2$ (epsilon) & -1.3906 & \\
\hline Dolomite-ord & 0.1485 & s/sat & Dol & omite-dis & -1.4333 & \\
\hline Coesite & 0.1027 & s/sat & $\mathrm{Zn}($ & $\mathrm{OH}) 2$ (beta) & -1.6665 & \\
\hline Cristobalite(bet & -0.0941 & & Chr & ysotile & -1.9685 & \\
\hline Ice & -0.1194 & & Flu & orite & -2.2627 & \\
\hline Calcite & -0.2538 & & Tre & molite & -2.4645 & \\
\hline $\begin{array}{l}\text { Aragonite } \\
\text { (only minerals }\end{array}$ & $\begin{array}{l}-0.3983 \\
\text { with } \log \end{array}$ & $Q / K>$ & $\begin{array}{l}\text { Ens } \\
3 \text { li }\end{array}$ & $\begin{array}{l}\text { tatite } \\
\text { sted) }\end{array}$ & -2.6961 & \\
\hline Gases & fugac & ity & 10 & $g$ fug. & & \\
\hline - - - - - - - - - - - - - & 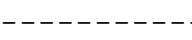 & ---- & --- & $-\ldots-\ldots$ & & \\
\hline $02(g)$ & $\odot$ & .1755 & & -0.756 & & \\
\hline $\mathrm{H} 2 \mathrm{O}(\mathrm{g})$ & $\odot$ & 01903 & & -1.721 & & \\
\hline $\mathrm{co2}(\mathrm{g})$ & $\odot . \odot \odot$ & 07859 & & -3.105 & & \\
\hline $\mathrm{HF}(\mathrm{g})$ & 1.777 & e- -014 & & 13.750 & & \\
\hline $\mathrm{HCl}(\mathrm{g})$ & 6.854 & $e-020$ & & 19.164 & & \\
\hline N02 $(g)$ & 2.888 & $e-021$ & & 20.539 & & \\
\hline $\mathrm{N} 2(\mathrm{~g})$ & 3.262 & $e-022$ & & 21.486 & & \\
\hline NO (g) & 2.888 & $e-027$ & & 26.539 & & \\
\hline $\operatorname{cl} 2(\mathrm{~g})$ & 5.786 & e- -31 & & 30.238 & & \\
\hline $\operatorname{SiF} 4(g)$ & 1.252 & $e-038$ & & 37.902 & & \\
\hline $\mathrm{H} 2(\mathrm{~g})$ & 8.946 & $e-043$ & & 42.048 & & \\
\hline $\mathrm{co}(\mathrm{g})$ & 2.246 & $e-049$ & & 48.649 & & \\
\hline $\mathrm{S} 02(\mathrm{~g})$ & 7.344 & $e-058$ & & 57.134 & & \\
\hline $\mathrm{NH3}(\mathrm{g})$ & 1.609 & e- -071 & & 70.793 & & \\
\hline $\mathrm{Zn}(\mathrm{g})$ & 1.660 & e- -075 & & 74.780 & & \\
\hline $\mathrm{Na}(\mathrm{g})$ & 3.848 & e- -077 & & 76.415 & & \\
\hline $\mathrm{F} 2(\mathrm{~g})$ & 2.505 & $e-084$ & & 83.601 & & \\
\hline$M g(g)$ & 8.606 & e-132 & & 31.065 & & \\
\hline $\mathrm{H} 2 \mathrm{~S}(\mathrm{~g})$ & 1.041 & $e-146$ & & 45.983 & & \\
\hline $\mathrm{CH} 4(\mathrm{~g})$ & 2.384 & $e-148$ & & 47.623 & & \\
\hline $\mathrm{Ca}(\mathrm{g})$ & 1.929 & $e-154$ & & 53.715 & & \\
\hline$C(g)$ & 4.236 & $e-193$ & & 92.373 & & \\
\hline $\operatorname{Si}(g)$ & 3.327 & $e-224$ & & 23.478 & & \\
\hline $\mathrm{S} 2(\mathrm{~g})$ & 9.172 & $e-235$ & & 34.038 & & \\
\hline $\mathrm{C} 2 \mathrm{H} 4(\mathrm{~g})$ & 3.392 & $e-242$ & & 41.469 & & \\
\hline & & & $\mathrm{n} f l$ & uid & Sorbed & $\mathrm{Kd}$ \\
\hline Original basis to & otal moles & mol & & $\mathrm{mg} / \mathrm{kg}$ & moles $\quad \mathrm{mg} / \mathrm{kg}$ & $\mathrm{L} / \mathrm{kg}$ \\
\hline - - - - - - - - - - - - - - & 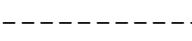 &.---- & --- & $-\ldots$ & 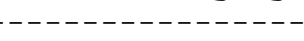 & $-\ldots$ \\
\hline $\mathrm{Ba}++$ & 8. 81e- - ๑८ & $8.81 \mathrm{e}$ & $\odot \odot 8$ & ๑. 0121 & & \\
\hline $\mathrm{Ca}++$ & 0.000304 & $0.0 \odot$ & 304 & 12.2 & & \\
\hline $\mathrm{Cl}-$ & 3. ๑०e-๑०5 & $3 . \odot \odot \mathrm{e}$ & $\odot \odot 5$ & 1.06 & & \\
\hline $\mathrm{F}-$ & $4.57 e-005$ & $4.57 \mathrm{e}$ & 005 & 0.869 & & \\
\hline $\mathrm{H}+$ & 1. $05 e-005$ & $-1.05 e$ & $0 \odot 5$ & -0.0105 & & \\
\hline $\mathrm{H} 2 \mathrm{O}$ & 55.5 & & 5.5 & $1.00 \mathrm{e}+\odot \odot 6$ & & \\
\hline $\mathrm{HCO} 3-$ & $\odot .00172$ & 0.0 & 172 & 105 & & \\
\hline $\mathrm{Mg}++$ & $9.51 \mathrm{e}-0 \odot 5$ & $9.51 \mathrm{e}$ & $\odot \odot 5$ & 2.31 & & \\
\hline Mo04 - - & $5.33 e-0 \odot 8$ & $5.33 \mathrm{e}$ & $\odot \odot 8$ & 0.00853 & & \\
\hline $\mathrm{NH3}(\mathrm{aq})$ & $1.86 e-005$ & $1.86 \mathrm{e}$ & $\odot \odot 5$ & 0.316 & & \\
\hline $\mathrm{Na}+$ & $\odot . \odot \odot \odot 878$ & $\odot . \odot \odot$ & 878 & 20.2 & & \\
\hline $02(\mathrm{aq})$ & $\odot .000283$ & 0.00 & 283 & 9.06 & & \\
\hline Re04- & $2.14 \mathrm{e}-006$ & $2.14 \mathrm{e}$ & 006 & 0.534 & & \\
\hline SO4-- & 0.000110 & 0.00 & 110 & 10.5 & & \\
\hline Si02(aq) & 0.000631 & 0.00 & 631 & 37.9 & & \\
\hline $\mathrm{Zn}++$ & $2.71 \mathrm{e}-006$ & $2.71 \mathrm{e}$ & $\odot \odot 6$ & 0.177 & & \\
\hline
\end{tabular}




\begin{tabular}{|c|c|c|c|c|c|}
\hline \multicolumn{2}{|c|}{ Elemental composition } & \multicolumn{2}{|c|}{ In fluid } & \multicolumn{2}{|c|}{ Sorbed } \\
\hline & total moles & moles & $\mathrm{mg} / \mathrm{kg}$ & moles & $\mathrm{mg} / \mathrm{kg}$ \\
\hline$-n_{-}$ & 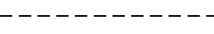 & - - - - - - - - - & 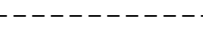 & & \\
\hline Barium & 8. 814e- -๑8 & $8.814 \mathrm{e}-\odot \odot 8$ & 0.01210 & & \\
\hline Calcium & $\odot .0003045$ & 0.0003045 & 12.20 & & \\
\hline Carbon & $\odot .001724$ & ๑. . 001724 & 20.71 & & \\
\hline Chlorine & $2.998 \mathrm{e}-005$ & $2.998 \mathrm{e}-005$ & 1.063 & & \\
\hline Fluorine & $4.574 \mathrm{e}-005$ & $4.574 \mathrm{e}-005$ & 0.8688 & & \\
\hline Hydrogen & 111.0 & 111.0 & $1.119 \mathrm{e}+005$ & & \\
\hline Magnesium & $9.515 e-005$ & $9.515 e-005$ & 2.312 & & \\
\hline Molybdenum & $5.334 \mathrm{e}-008$ & $5.334 e-008$ & $\odot .005116$ & & \\
\hline Nitrogen & $1.857 \mathrm{e}-005$ & $1.857 \mathrm{e}-005$ & $\odot .2600$ & & \\
\hline oxygen & 55.52 & 55.52 & $8.880 e+005$ & & \\
\hline Rhenium & $2.135 \mathrm{e}-0 \odot 6$ & $2.135 \mathrm{e}-006$ & 0.3975 & & \\
\hline Silicon & $\odot .0006307$ & $\odot .0006307$ & 17.71 & & \\
\hline Sodium & $\odot .0 \odot \odot 8775$ & $\odot .0 \odot \odot 8775$ & $2 \odot .17$ & & \\
\hline Sulfur & ๑. . $00 \odot 1096$ & ๑. . 0001096 & 3.514 & & \\
\hline Zinc & $2.709 \mathrm{e}-006$ & $2.709 e-006$ & 0.1771 & & \\
\hline
\end{tabular}




\section{Sample D11NWTOP}

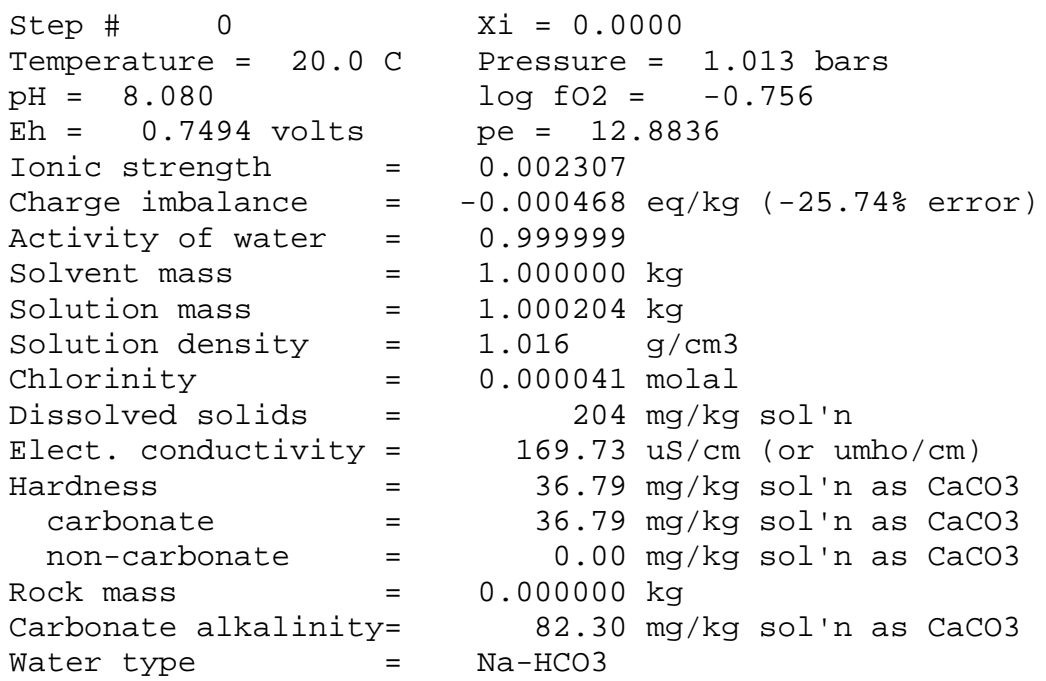

No minerals in system.

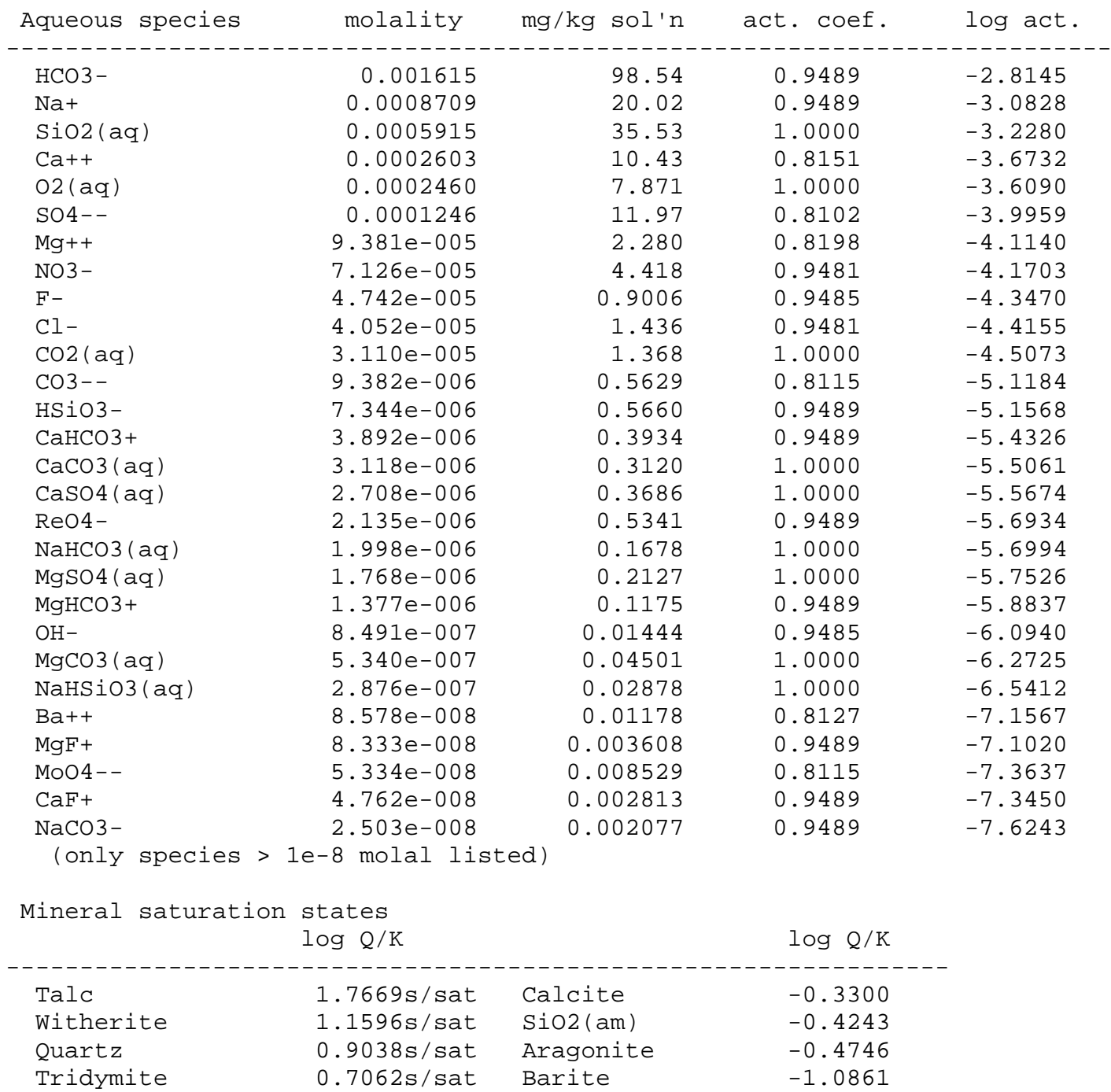




$\begin{array}{llll}\text { Cristobalite(alp } & 0.3418 \mathrm{~s} / \mathrm{sat} & \text { Monohydrocalcite } & -1.1508 \\ \text { Chalcedony } & 0.3279 \mathrm{~s} / \mathrm{sat} & \text { Magnesite } & -1.2718 \\ \text { Coesite } & 0.0806 \mathrm{~s} / \mathrm{sat} & \text { Dolomite-dis } & -1.5236 \\ \text { Dolomite } & 0.0582 \mathrm{~s} / \mathrm{sat} & \text { Chrysotile } & -2.0413 \\ \text { Dolomite-ord } & 0.0582 \mathrm{~s} / \mathrm{sat} & \text { Fluorite } & -2.2803 \\ \text { Cristobalite(bet } & -0.1162 & \text { Enstatite } & -2.7277 \\ \text { Ice } & -0.1194 & \text { Tremolite } & -2.8325\end{array}$

(only minerals with $\log \mathrm{Q} / \mathrm{K}>-3$ listed)

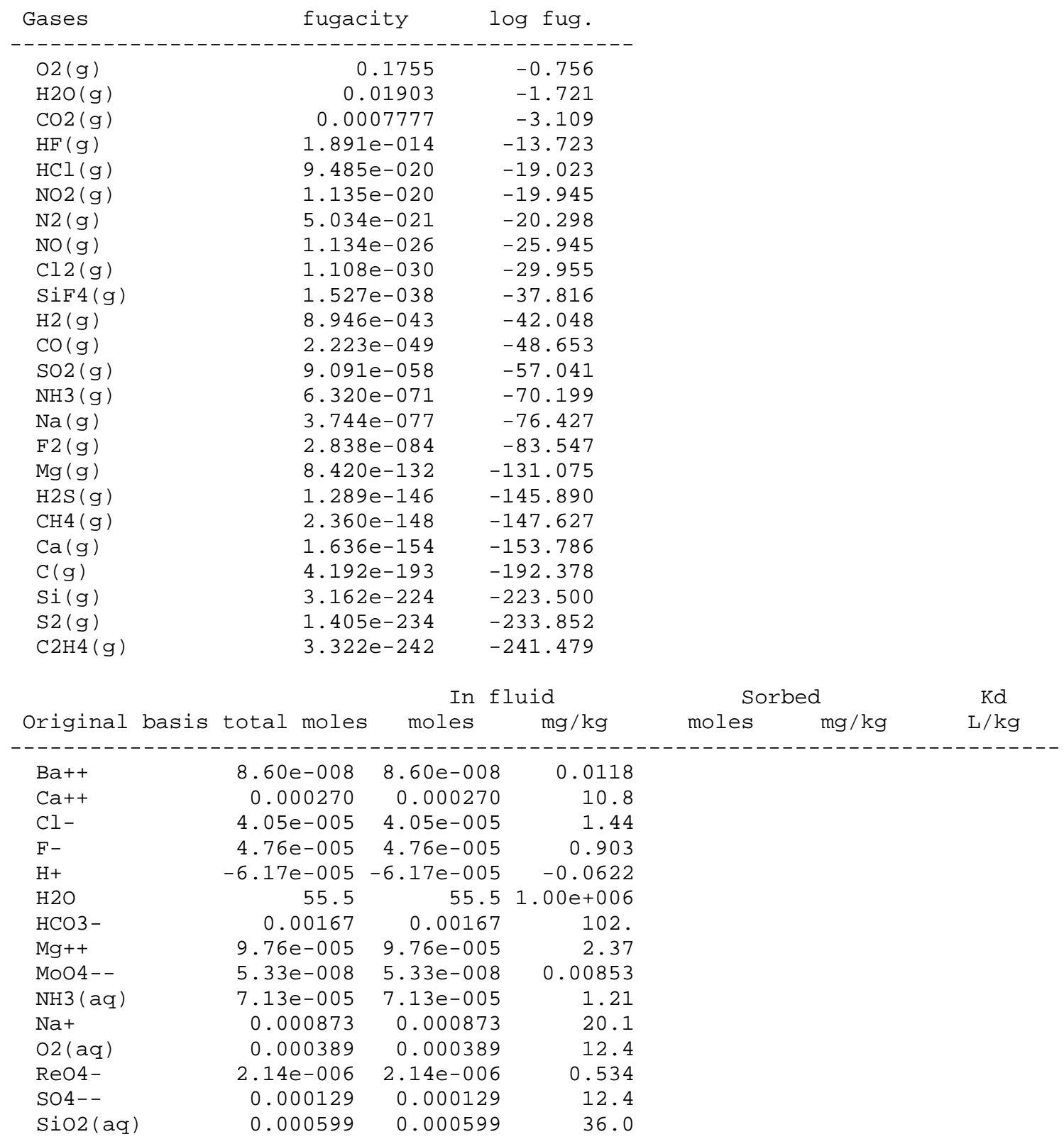

\begin{tabular}{lrccc}
\multicolumn{2}{c}{ Elemental composition } & \multicolumn{2}{c}{ In fluid } & \multicolumn{2}{c}{ Sorbed } \\
total moles & moles & $\mathrm{mg} / \mathrm{kg}$ & $\mathrm{moles}$ & $\mathrm{mg} / \mathrm{kg}$ \\
Barium & $8.599 \mathrm{e}-0 \odot 8$ & $8.599 \mathrm{e}-008$ & 0.01181 & \\
Calcium & $0.00 \odot 2701$ & $0.00 \odot 2701$ & 10.82 & \\
Carbon & 0.001667 & 0.001667 & 20.02 & \\
Chlorine & $4.053 \mathrm{e}-0 \odot 5$ & $4.053 \mathrm{e}-005$ & 1.436 & \\
Fluorine & $4.755 \mathrm{e}-0 \odot 5$ & $4.755 \mathrm{e}-005$ & 0.9032 &
\end{tabular}




$\begin{array}{lrrr}\text { Hydrogen } & 111.0 & 111 . \odot & 1.119 \mathrm{e}+005 \\ \text { Magnesium } & 9.758 \mathrm{e}-005 & 9.758 \mathrm{e}-005 & 2.371 \\ \text { Molybdenum } & 5.334 \mathrm{e}-008 & 5.334 \mathrm{e}-008 & 0.005116 \\ \text { Nitrogen } & 7.126 \mathrm{e}-005 & 7.126 \mathrm{e}-005 & 0.9979 \\ \text { Oxygen } & 55.52 & 55.52 & 8.880 \mathrm{e}+005 \\ \text { Rhenium } & 2.135 \mathrm{e}-006 & 2.135 \mathrm{e}-006 & 0.3975 \\ \text { Silicon } & 0.0005992 & 0.0005992 & 16.82 \\ \text { Sodium } & 0.0008732 & 0.0008732 & 20.07 \\ \text { Sulfur } & 0.0001291 & 0.0001291 & 4.138\end{array}$




\section{Sample D11NETOP}

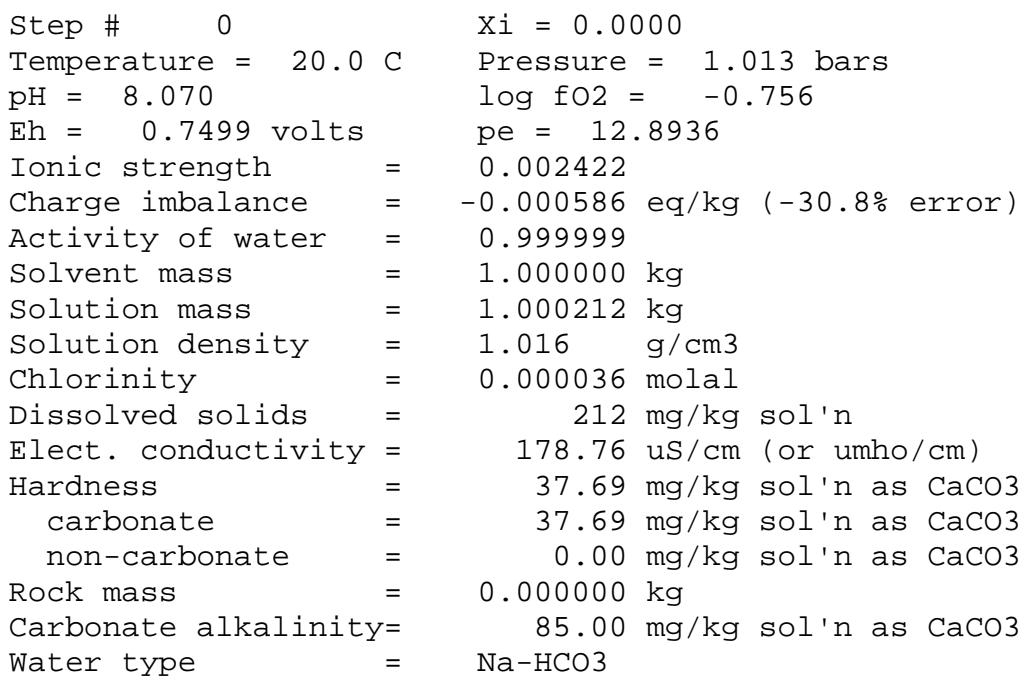

No minerals in system.

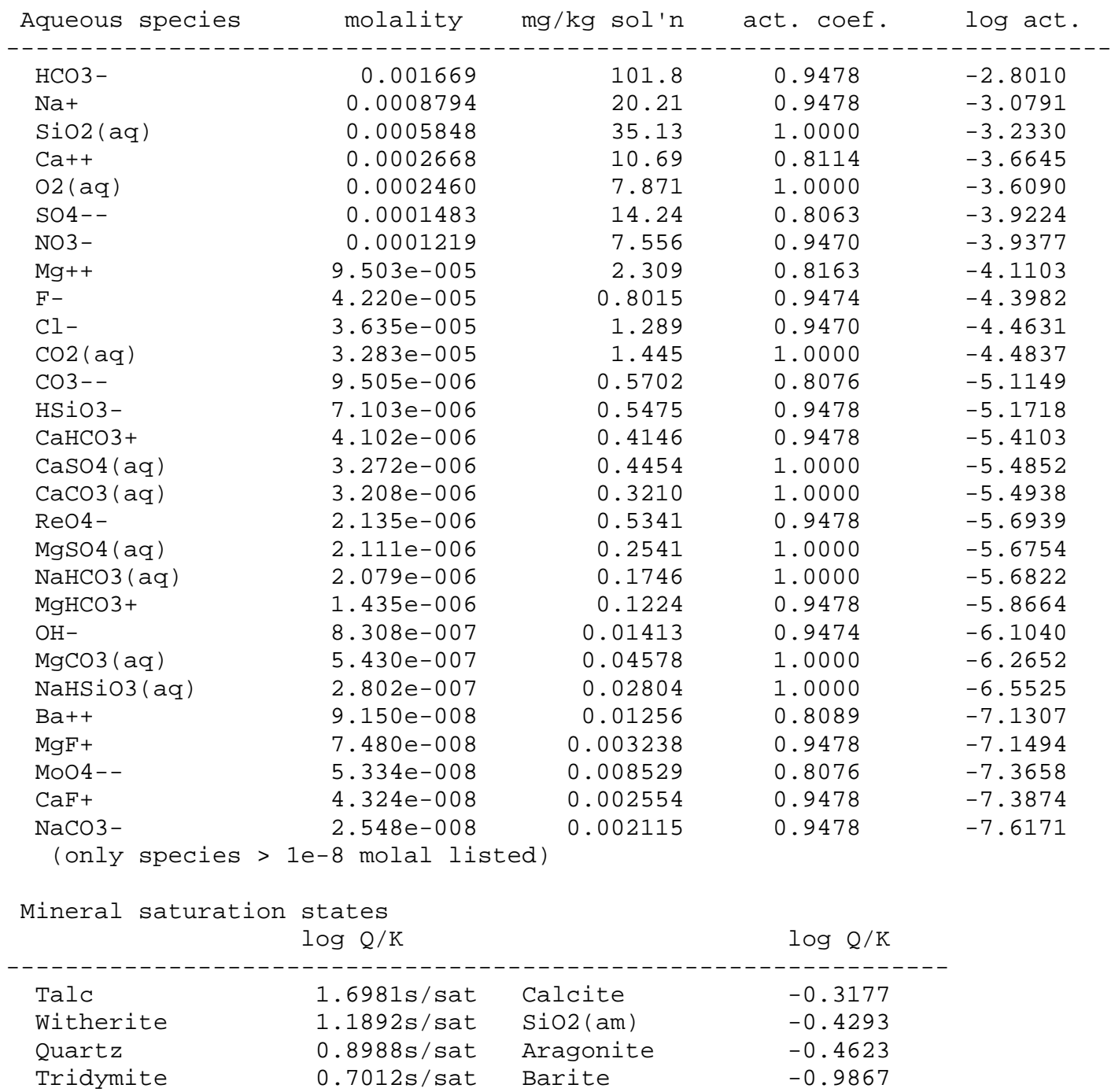




$\begin{array}{llll}\text { Cristobalite(alp } & 0.3368 \mathrm{~s} / \mathrm{sat} & \text { Monohydrocalcite } & -1.1385 \\ \text { Chalcedony } & \odot .3229 \mathrm{~s} / \mathrm{sat} & \text { Magnesite } & -1.2645 \\ \text { Dolomite } & 0.0778 \mathrm{~s} / \mathrm{sat} & \text { Dolomite-dis } & -1.5040 \\ \text { Dolomite-ord } & 0.0778 \mathrm{~s} / \mathrm{sat} & \text { Chrysotile } & -2.1001 \\ \text { Coesite } & 0.0756 \mathrm{~s} / \mathrm{sat} & \text { Fluorite } & -2.3739 \\ \text { Ice } & -0.1194 & \text { Enstatite } & -2.7490 \\ \text { Cristobalite(bet } & -\odot .1212 & \text { Tremolite } & -2.9763 \\ \quad \text { (only minerals with } \log \mathrm{Q} / \mathrm{K}>-3 \text { listed) } & \end{array}$

\begin{tabular}{lcc} 
Gases & fugacity & log fug \\
\hline O2 $(\mathrm{g})$ & 0.1755 & -0.756 \\
$\mathrm{H} 2 \mathrm{~g}(\mathrm{~g})$ & 0.01903 & -1.721 \\
$\mathrm{CO} 2(\mathrm{~g})$ & 0.0008211 & -3.086 \\
$\mathrm{HF}(\mathrm{g})$ & $1.720 \mathrm{e}-014$ & -13.764 \\
$\mathrm{HCl}(\mathrm{g})$ & $8.698 \mathrm{e}-020$ & -19.061 \\
$\mathrm{~N} 2(\mathrm{~g})$ & $1.983 \mathrm{e}-020$ & -19.703 \\
$\mathrm{~N} 2(\mathrm{~g})$ & $1.538 \mathrm{e}-020$ & -19.813 \\
$\mathrm{NO}(\mathrm{g})$ & $1.983 \mathrm{e}-026$ & -25.703 \\
$\mathrm{C} 12(\mathrm{~g})$ & $9.318 \mathrm{e}-031$ & -30.031 \\
$\mathrm{SiF}(\mathrm{g})$ & $1.033 \mathrm{e}-038$ & -37.986 \\
$\mathrm{H} 2(\mathrm{~g})$ & $8.946 \mathrm{e}-043$ & -42.048 \\
$\mathrm{CO}(\mathrm{g})$ & $2.347 \mathrm{e}-049$ & -48.630 \\
$\mathrm{~S} 2(\mathrm{~g})$ & $1.127 \mathrm{e}-057$ & -56.948 \\
$\mathrm{NH} 3(\mathrm{~g})$ & $1.105 \mathrm{e}-070$ & -69.957 \\
$\mathrm{Na}(\mathrm{g})$ & $3.690 \mathrm{e}-077$ & -76.433 \\
$\mathrm{~F} 2(\mathrm{~g})$ & $2.348 \mathrm{e}-084$ & -83.629 \\
$\mathrm{Mg}(\mathrm{g})$ & $8.111 \mathrm{e}-132$ & -131.091 \\
$\mathrm{H} 2 \mathrm{~g}(\mathrm{~g})$ & $1.598 \mathrm{e}-146$ & -145.796 \\
$\mathrm{CH} 4(\mathrm{~g})$ & $2.491 \mathrm{e}-148$ & -147.604 \\
$\mathrm{Ca}(\mathrm{g})$ & $1.594 \mathrm{e}-154$ & -153.798 \\
$\mathrm{C}(\mathrm{g})$ & $4.425 \mathrm{e}-193$ & -192.354 \\
$\mathrm{Si}(\mathrm{g})$ & $3.126 \mathrm{e}-224$ & -223.505 \\
$\mathrm{~S} 2(\mathrm{~g})$ & $2.161 \mathrm{e}-234$ & -233.665 \\
$\mathrm{C} 2 \mathrm{H} 4(\mathrm{~g})$ & $3.703 \mathrm{e}-242$ & -241.431 \\
& &
\end{tabular}

\begin{tabular}{|c|c|c|c|c|c|}
\hline \multirow[b]{2}{*}{ Original basis } & \multirow[b]{2}{*}{ total moles } & \multicolumn{2}{|c|}{ In fluid } & \multicolumn{2}{|l|}{ Sorbed } \\
\hline & & moles & $\mathrm{mg} / \mathrm{kg}$ & moles $\quad \mathrm{mg} / \mathrm{kg}$ & $\mathrm{L} / \mathrm{kg}$ \\
\hline - - - - - - - - - - & 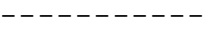 & ------- & $-\ldots-\ldots$ & - - - - - - & $-\cdots-\cdots$ \\
\hline $\mathrm{Ba}++$ & $9.17 \mathrm{e}-0 \odot 8$ & $9.17 \mathrm{e}-008$ & 0.0126 & & \\
\hline $\mathrm{Ca}++$ & 0.000277 & $\odot .000277$ & 11.1 & & \\
\hline $\mathrm{Cl}-$ & $3.64 \mathrm{e}-005$ & $3.64 \mathrm{e}-005$ & 1.29 & & \\
\hline $\mathrm{F}-$ & $4.23 e-005$ & $4.23 e-005$ & 0.804 & & \\
\hline $\mathrm{H}+$ & -0.000111 & -0.000111 & -0.111 & & \\
\hline $\mathrm{H} 2 \mathrm{O}$ & 55.5 & 55.5 & 1. $\odot \odot e+\odot \odot 6$ & & \\
\hline HCO3 - & 0.00172 & $\odot .00172$ & 105 & & \\
\hline $\mathrm{Mg}++$ & $9.92 e-005$ & $9.92 e-005$ & 2.41 & & \\
\hline Mo04 - - & $5.33 e-008$ & $5.33 e-008$ & $\odot .0 \odot 853$ & & \\
\hline NH3 (aq) & ๑. $0 \odot \odot 122$ & ๑. $0 \odot \odot 122$ & 2.08 & & \\
\hline $\mathrm{Na}+$ & ๑. .0००882 & $\odot .000882$ & 20.3 & & \\
\hline $02(\mathrm{aq})$ & $\odot . \odot \odot \odot 49 \odot$ & $\odot . \odot \odot \odot 49 \odot$ & 15.7 & & \\
\hline Re04 - & $2.14 \mathrm{e}-\odot \odot 6$ & $2.14 \mathrm{e}-006$ & 0.534 & & \\
\hline SO4 - - & $\odot .000154$ & $\odot .000154$ & 14.8 & & \\
\hline $\operatorname{si02}(\mathrm{aq})$ & $\odot .000592$ & $\odot .000592$ & 35.6 & & \\
\hline
\end{tabular}

\begin{tabular}{|c|c|c|c|c|c|}
\hline \multicolumn{2}{|c|}{ composition } & \multicolumn{2}{|c|}{ In fluid } & \multicolumn{2}{|c|}{ Sorbed } \\
\hline & total moles & moles & $\mathrm{mg} / \mathrm{kg}$ & moles & $\mathrm{mg} / \mathrm{kg}$ \\
\hline & 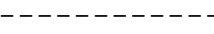 & - - - - - - - - - & - - $_{-}$ & & \\
\hline Barium & $9.173 e-008$ & $9.173 e-008$ & 0.01259 & & \\
\hline Calcium & $\odot .0002775$ & $\odot .0002775$ & 11.12 & & \\
\hline Carbon & ๑. 001722 & ๑. 001722 & 20.68 & & \\
\hline Chlorine & $3.636 e-005$ & $3.636 e-005$ & 1.289 & & \\
\hline Fluorine & $4.232 e-005$ & $4.232 e-005$ & 0.8038 & & \\
\hline
\end{tabular}




$\begin{array}{lrrr}\text { Hydrogen } & 111.0 & 111.0 & 1.119 \mathrm{e}+005 \\ \text { Magnesium } & 9.920 \mathrm{e}-005 & 9.920 \mathrm{e}-005 & 2.411 \\ \text { Molybdenum } & 5.334 \mathrm{e}-008 & 5.334 \mathrm{e}-008 & 0.005116 \\ \text { Nitrogen } & 0.0001219 & 0.0001219 & 1.707 \\ \text { Oxygen } & 55.52 & 55.52 & 8.880 \mathrm{e}+005 \\ \text { Rhenium } & 2.135 \mathrm{e}-006 & 2.135 \mathrm{e}-006 & 0.3975 \\ \text { Silicon } & 0.0005922 & 0.0005922 & 16.63 \\ \text { Sodium } & 0.0008818 & 0.0008818 & 20.27 \\ \text { Sulfur } & 0.0001537 & 0.0001537 & 4.926\end{array}$




\section{Sample D11SETOP}

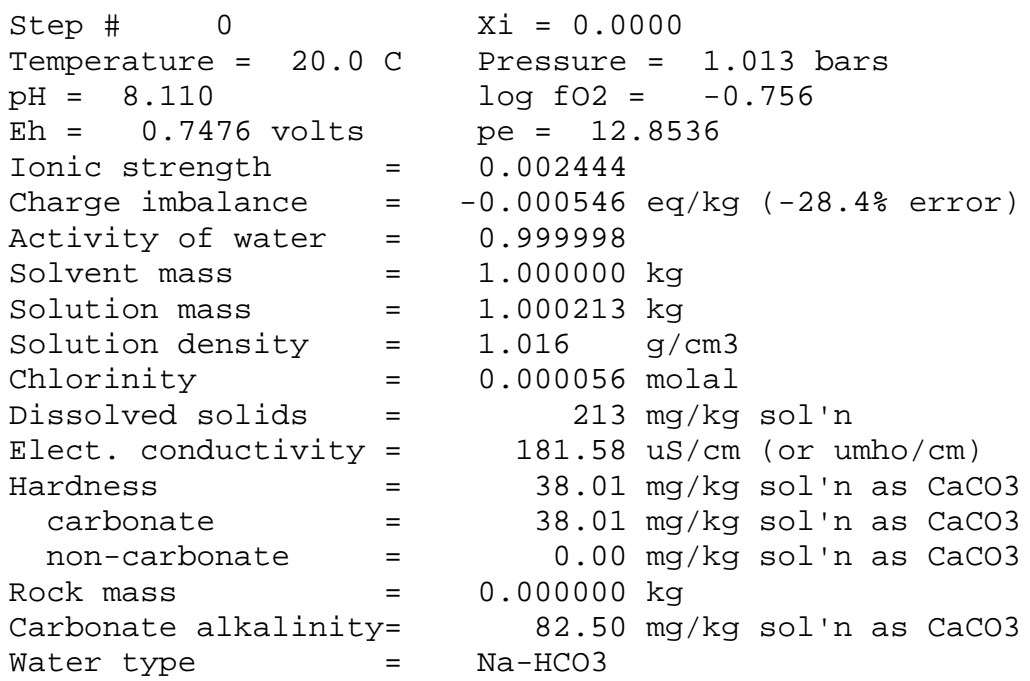

No minerals in system.

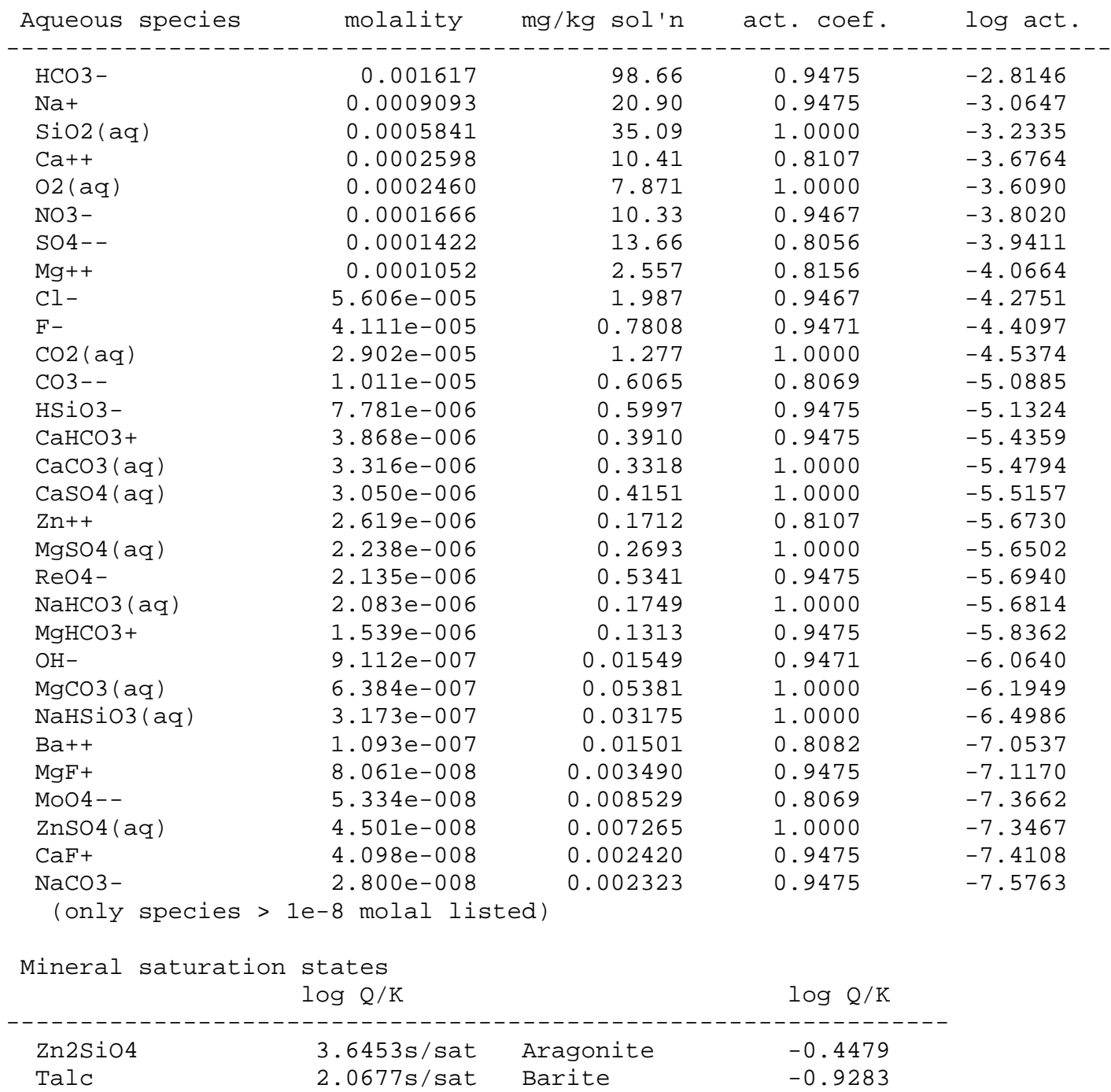




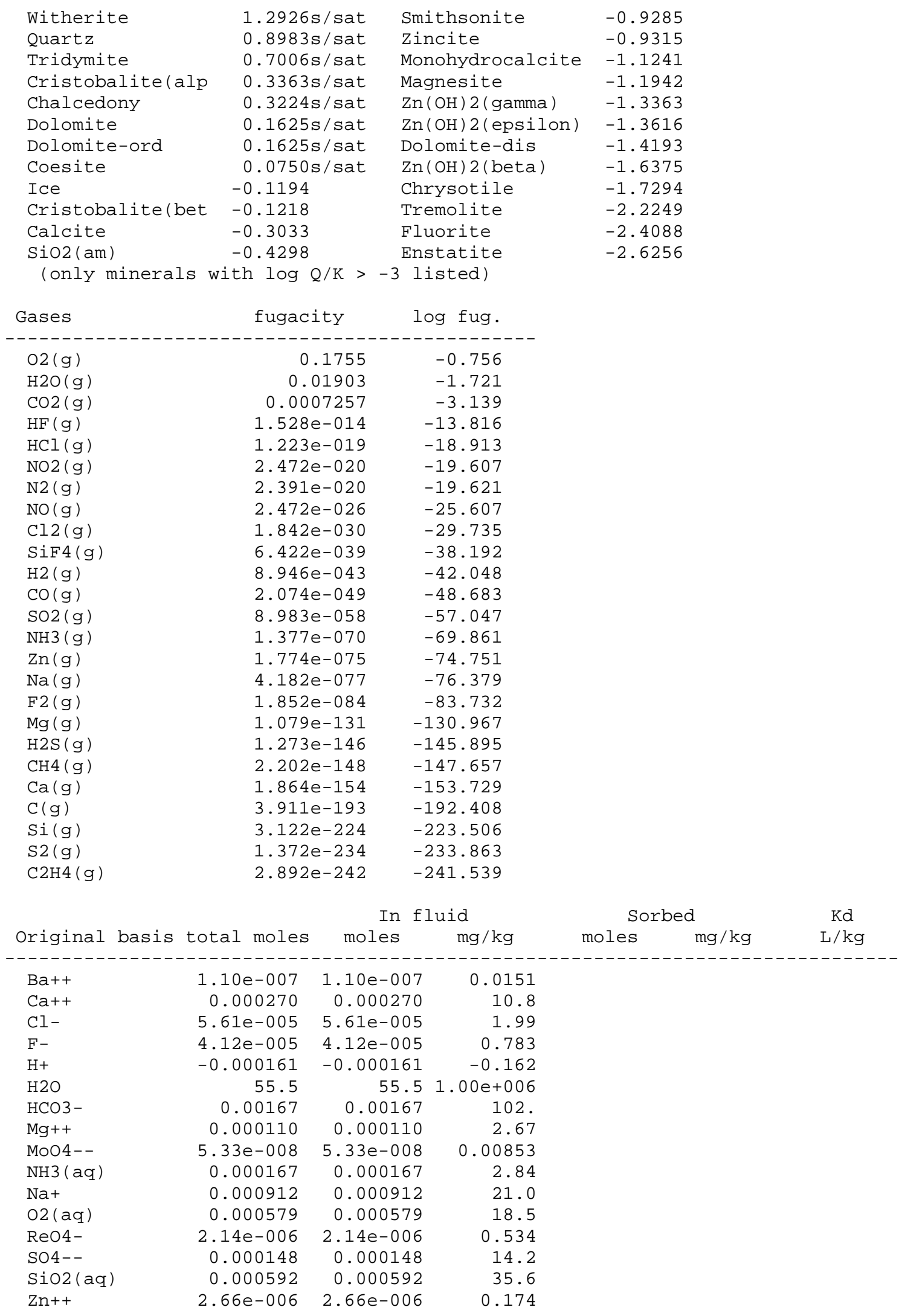




\begin{tabular}{|c|c|c|c|c|c|}
\hline \multicolumn{2}{|c|}{ Elemental composition } & \multicolumn{2}{|c|}{ In fluid } & \multicolumn{2}{|c|}{ Sorbed } \\
\hline & total moles & moles & $\mathrm{mg} / \mathrm{kg}$ & moles & $\mathrm{mg} / \mathrm{kg}$ \\
\hline$-\ldots$ & - - - - - - - - - - & - - - - - - - - - & 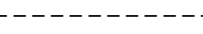 & 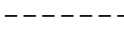 & \\
\hline Barium & 1. $.996-\odot \odot 7$ & 1. $096 \mathrm{e}-\odot \odot 7$ & 0.01505 & & \\
\hline Calcium & $\odot .0002701$ & 0.0002701 & 10.82 & & \\
\hline Carbon & ๑. . 001668 & ๑. . 001668 & 20.03 & & \\
\hline Chlorine & $5.607 \mathrm{e}-005$ & $5.607 e-005$ & 1.987 & & \\
\hline Fluorine & $4.123 e-005$ & $4.123 e-005$ & 0.7832 & & \\
\hline Hydrogen & 111.0 & 111.0 & $1.119 \mathrm{e}+005$ & & \\
\hline Magnesium & 0.0001097 & 0.0001097 & 2.666 & & \\
\hline Molybdenum & $5.334 \mathrm{e}-008$ & $5.334 e-008$ & $\odot .005116$ & & \\
\hline Nitrogen & $\odot .0 \odot \odot 1666$ & $\odot .0 \odot \odot 1666$ & 2.334 & & \\
\hline oxygen & 55.52 & 55.52 & $8.880 e+005$ & & \\
\hline Rhenium & $2.135 \mathrm{e}-0 \odot 6$ & $2.135 \mathrm{e}-006$ & 0.3975 & & \\
\hline Silicon & $\odot .0 \odot \odot 5922$ & $\odot .0005922$ & 16.63 & & \\
\hline Sodium & ๑. . 0009118 & ๑. . 0009118 & 20.96 & & \\
\hline Sulfur & $\odot .0 \odot \odot 1475$ & ๑. . 001475 & 4.729 & & \\
\hline Zinc & $2.664 \mathrm{e}-006$ & $2.664 \mathrm{e}-006$ & 0.1741 & & \\
\hline
\end{tabular}




\section{Sample D1419MMT_ts}

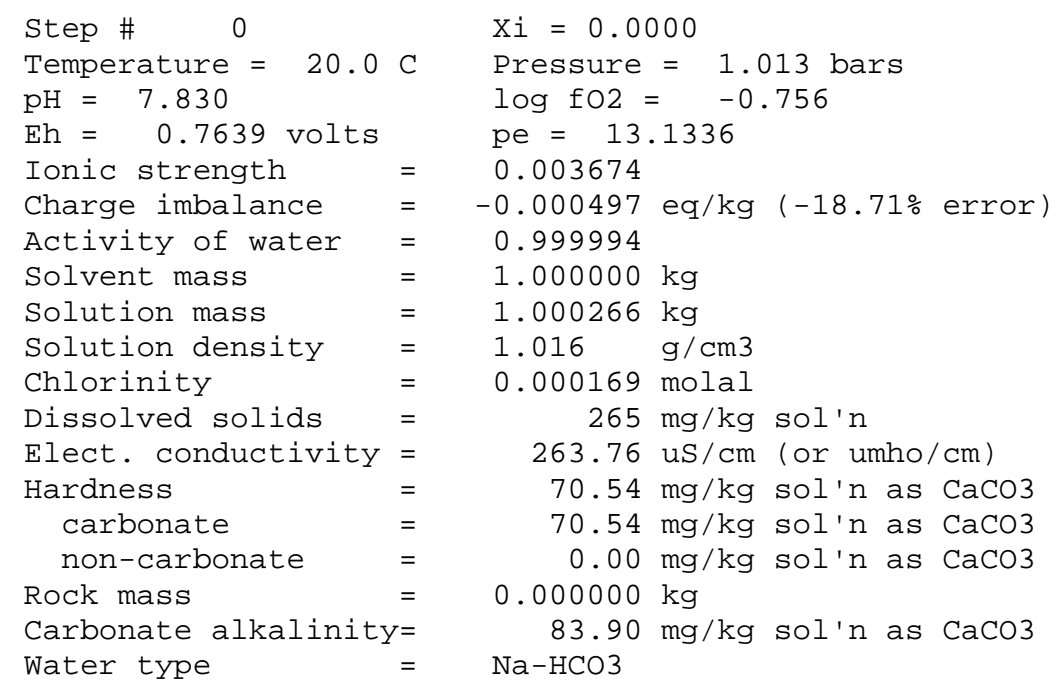

No minerals in system.

\begin{tabular}{|c|c|c|c|c|}
\hline Aqueous species & molality & $\mathrm{mg} / \mathrm{kg}$ sol'n & act. coef. & $\log$ act \\
\hline - - - - - - - - - - - - & ------- & 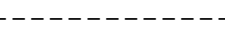 & 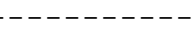 & ---- \\
\hline HCO3 - & ๑. $0 \odot 1651$ & 100.7 & $\odot .9370$ & -2.8106 \\
\hline $\mathrm{Na}+$ & $\odot .0009864$ & 22.67 & $\odot .9370$ & -3.0342 \\
\hline $\operatorname{Si02}(\mathrm{aq})$ & 0.0005009 & 30.09 & 1.0000 & -3.3003 \\
\hline $\mathrm{Ca}++$ & 0.0004763 & 19.08 & 0.7771 & -3.4316 \\
\hline N03 - & ๑. . $0 \odot 3889$ & 24.10 & $\odot .9358$ & -3.4390 \\
\hline SO4 - - & ๑. .๑०3372 & 32.38 & $\odot .7699$ & -3.5857 \\
\hline $02(\mathrm{aq})$ & $\odot .0002460$ & 7.871 & 1.0000 & -3.6090 \\
\hline $\mathrm{Mg}++$ & ๑. . 0001941 & 4.716 & 0.7839 & -3.8177 \\
\hline Cl- & ๑. . 0001687 & 5.980 & $\odot .9358$ & -3.8017 \\
\hline $\mathrm{K}+$ & $6.732 \mathrm{e}-\odot \odot 5$ & 2.632 & 0.9358 & -4.2006 \\
\hline $\mathrm{co2}(\mathrm{aq})$ & $5.580 \mathrm{e}-005$ & 2.455 & 1.0000 & -4.2534 \\
\hline CaSO4( aq) & $1.215 \mathrm{e}-\odot \odot 5$ & 1.653 & $1.0 \odot \odot \odot$ & -4.9155 \\
\hline $\operatorname{MgSO} 4(\mathrm{aq})$ & $8.993 e-\odot \odot 6$ & 1.082 & 1.0000 & -5.0461 \\
\hline Re04 - & $8.562 \mathrm{e}-006$ & 2.142 & 0.9370 & -5.0957 \\
\hline CaHCO3+ & $6.936 \mathrm{e}-\odot \odot 6$ & 0.7011 & $\odot .9370$ & -5.1871 \\
\hline C03-- & $5.597 \mathrm{e}-\odot \odot 6$ & 0.3358 & 0.7718 & -5.3646 \\
\hline HSi03- & $3.541 \mathrm{e}-006$ & 0.2729 & 0.9370 & -5.4791 \\
\hline $\mathrm{CaC03}(\mathrm{aq})$ & $3.086 e-\odot \odot 6$ & 0.3088 & 1.0000 & -5.5106 \\
\hline $\mathrm{MgHCO} 3+$ & $2.784 \mathrm{e}-\odot \odot 6$ & 0.2375 & 0.9370 & -5.5835 \\
\hline $\mathrm{Zn++}$ & $2.452 \mathrm{e}-\odot \odot 6$ & 0.1603 & 0.7771 & -5.7201 \\
\hline $\mathrm{NaHCO} 3(\mathrm{aq})$ & $2.254 \mathrm{e}-\odot \odot 6$ & 0.1893 & 1.0000 & -5.6470 \\
\hline $\operatorname{MgC0} 3(a q)$ & $5.994 \mathrm{e}-\odot \odot 7$ & 0.05052 & 1.0000 & -6.2223 \\
\hline $\mathrm{OH}-$ & $4.837 \mathrm{e}-\odot \odot 7$ & ๑. .0०8224 & 0.9364 & -6.3440 \\
\hline $\mathrm{Ba}++$ & $1.553 \mathrm{e}-\odot \odot 7$ & ๑. . 02132 & 0.7736 & -6.9203 \\
\hline NaHSi03(aq) & $1.532 \mathrm{e}-\odot \odot 7$ & 0.01533 & $1.000 \odot$ & -6.8148 \\
\hline MoO4- - & 1. $364 \mathrm{e}-\odot \odot 7$ & ๑. .02181 & 0.7718 & -6.9776 \\
\hline KSO4 - & $1.327 \mathrm{e}-\odot \odot 7$ & 0.01793 & 0.9370 & -6.9055 \\
\hline Znso4(aq) & $9.154 \mathrm{e}-\odot \odot 8$ & $\odot .01478$ & $1.0 \odot \odot \odot$ & -7.0384 \\
\hline $\mathrm{NaCl}(\mathrm{aq})$ & $2.379 \mathrm{e}-\odot \odot 8$ & $\odot .001390$ & 1.0000 & -7.6236 \\
\hline $\mathrm{MgCl}+$ & $1.948 \mathrm{e}-\odot \odot 8$ & $\odot .001164$ & $\odot .9370$ & -7.7386 \\
\hline $\mathrm{NaCO} 3-$ & 1. $608 \mathrm{e}-\odot \odot 8$ & 0.001335 & 0.9370 & -7.8219 \\
\hline $\mathrm{H}+$ & $1.57 \odot \mathrm{e}-\odot \odot 8$ & $1.582 \mathrm{e}-005$ & 0.9422 & -7.8300 \\
\hline $\mathrm{CaCl}+$ & 1. $264 \mathrm{e}-\odot \odot 8$ & ๑. .๑๑9542 & $\odot .9370$ & -7.9267 \\
\hline
\end{tabular}




\begin{tabular}{|c|c|c|c|}
\hline Mineral saturatio & \multicolumn{2}{|c|}{$\log Q / K$} & $\log \mathrm{Q} / \mathrm{K}$ \\
\hline Zn2Si04 & $2.3645 \mathrm{~s} / \mathrm{sat}$ & Aragonite & $-\odot .4791$ \\
\hline Witherite & $1.1499 \mathrm{~s} / \mathrm{sat}$ & SiO2( $(\mathrm{am})$ & -0.4966 \\
\hline Talc & $0.8668 \mathrm{~s} / \mathrm{sat}$ & Monohydrocalcite & -1.1553 \\
\hline Quartz & $0.8316 \mathrm{~s} / \mathrm{sat}$ & Magnesite & -1.2216 \\
\hline Tridymite & $0.6339 \mathrm{~s} / \mathrm{sat}$ & Smithsonite & -1.2515 \\
\hline Cristobalite(alp & $0.2696 \mathrm{~s} / \mathrm{sat}$ & Dolomite-dis & -1.4779 \\
\hline Chalcedony & $0.2556 \mathrm{~s} / \mathrm{sat}$ & Zincite & -1.5385 \\
\hline Dolomite & $0.1039 \mathrm{~s} / \mathrm{sat}$ & $\mathrm{Zn}(\mathrm{OH}) 2($ gamma $)$ & -1.9433 \\
\hline Dolomite-ord & $0.1039 \mathrm{~s} / \mathrm{sat}$ & $\mathrm{Zn}(\mathrm{OH}) 2$ (epsilon) & -1.9686 \\
\hline Coesite & $\odot .0083 \mathrm{~s} / \mathrm{sat}$ & $\mathrm{Zn}(\mathrm{OH}) 2($ beta $)$ & -2.2445 \\
\hline Ice & $-\odot .1194$ & Gypsum & -2.5269 \\
\hline Cristobalite(bet & -0.1885 & Anhydrite & -2.7517 \\
\hline Calcite & -0.3346 & Chrysotile & -2.7969 \\
\hline Barite & -0.4396 & & \\
\hline (only minerals & vith $\log Q / K>$ & 3 listed) & \\
\hline \multicolumn{4}{|c|}{ Gases $\quad$ fugacity $\quad \log$ fug. } \\
\hline \multicolumn{4}{|c|}{ - - - - - - - - - - - - - - - - - - - - - - - } \\
\hline $02(g)$ & 0.1755 & -0.756 & \\
\hline $\mathrm{H} 20(\mathrm{~g})$ & 0.01903 & -1.721 & \\
\hline $\operatorname{co2}(\mathrm{g})$ & $\odot .001395$ & -2.855 & \\
\hline $\mathrm{HCl}(\mathrm{g})$ & $6.932 e-019$ & -18.159 & \\
\hline $\mathrm{N} 2(\mathrm{~g})$ & $4.617 e-019$ & -18.336 & \\
\hline N02 (g) & 1. $087 e-019$ & -18.964 & \\
\hline $\mathrm{NO}(\mathrm{g})$ & $1.086 \mathrm{e}-025$ & -24.964 & \\
\hline Cl2(g) & $5.919 e-029$ & -28.228 & \\
\hline $\mathrm{H} 2(\mathrm{~g})$ & $8.945 e-043$ & -42.048 & \\
\hline $\mathrm{co}(\mathrm{g})$ & $3.988 e-049$ & -48.399 & \\
\hline $\mathrm{SO} 2(\mathrm{~g})$ & $7.393 e-057$ & -56.131 & \\
\hline NH3 $(\mathrm{g})$ & $6.052 \mathrm{e}-070$ & -69.218 & \\
\hline $\mathrm{Zn}(\mathrm{g})$ & $4.385 e-076$ & -75.358 & \\
\hline $\mathrm{Na}(\mathrm{g})$ & $2.354 \mathrm{e}-077$ & -76.628 & \\
\hline$K(g)$ & $3.221 e-079$ & -78.492 & \\
\hline $\operatorname{Mg}(g)$ & $5.268 e-132$ & -131.278 & \\
\hline $\mathrm{H} 2 \mathrm{~S}(\mathrm{~g})$ & 1. $048 \mathrm{e}-145$ & -144.980 & \\
\hline $\mathrm{CH} 4(\mathrm{~g})$ & $4.234 \mathrm{e}-148$ & -147.373 & \\
\hline $\mathrm{Ca}(\mathrm{g})$ & $9.022 e-155$ & -154.045 & \\
\hline$C(g)$ & $7.520 \mathrm{e}-193$ & -192.124 & \\
\hline $\mathrm{Si}(\mathrm{g})$ & $2.677 \mathrm{e}-224$ & -223.572 & \\
\hline S2(g) & $9.293 e-233$ & -232.032 & \\
\hline $\mathrm{C} 2 \mathrm{H} 4(\mathrm{~g})$ & $1.069 e-241$ & -240.971 & \\
\hline
\end{tabular}




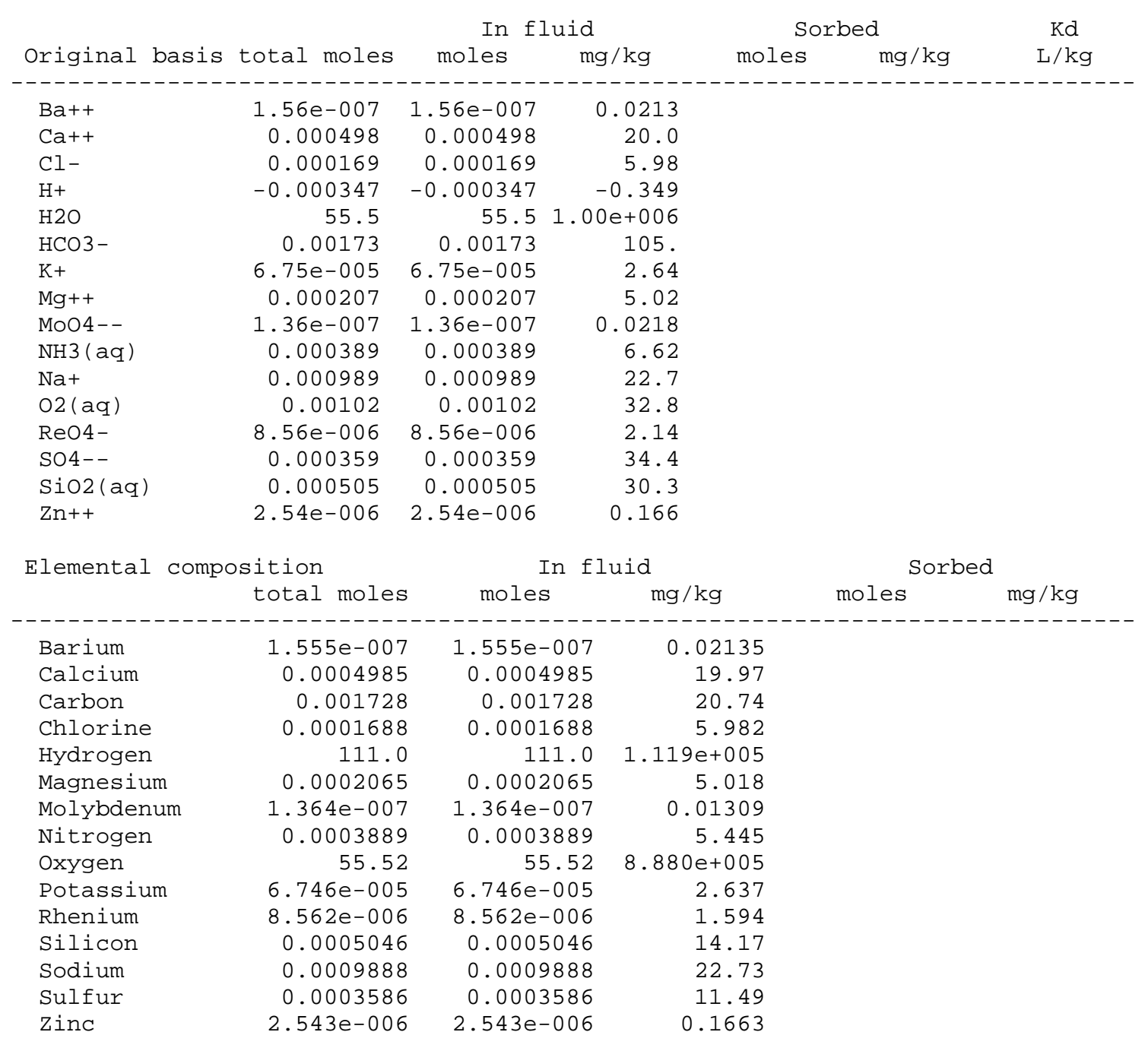




\section{Sample D1419MMT_bs}

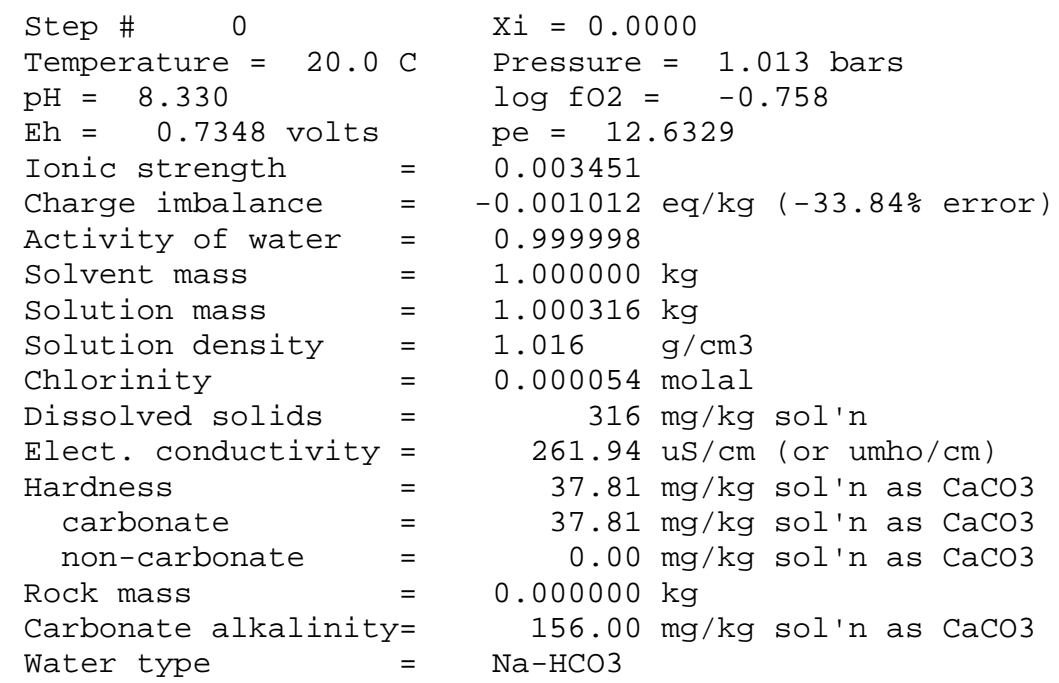

No minerals in system.

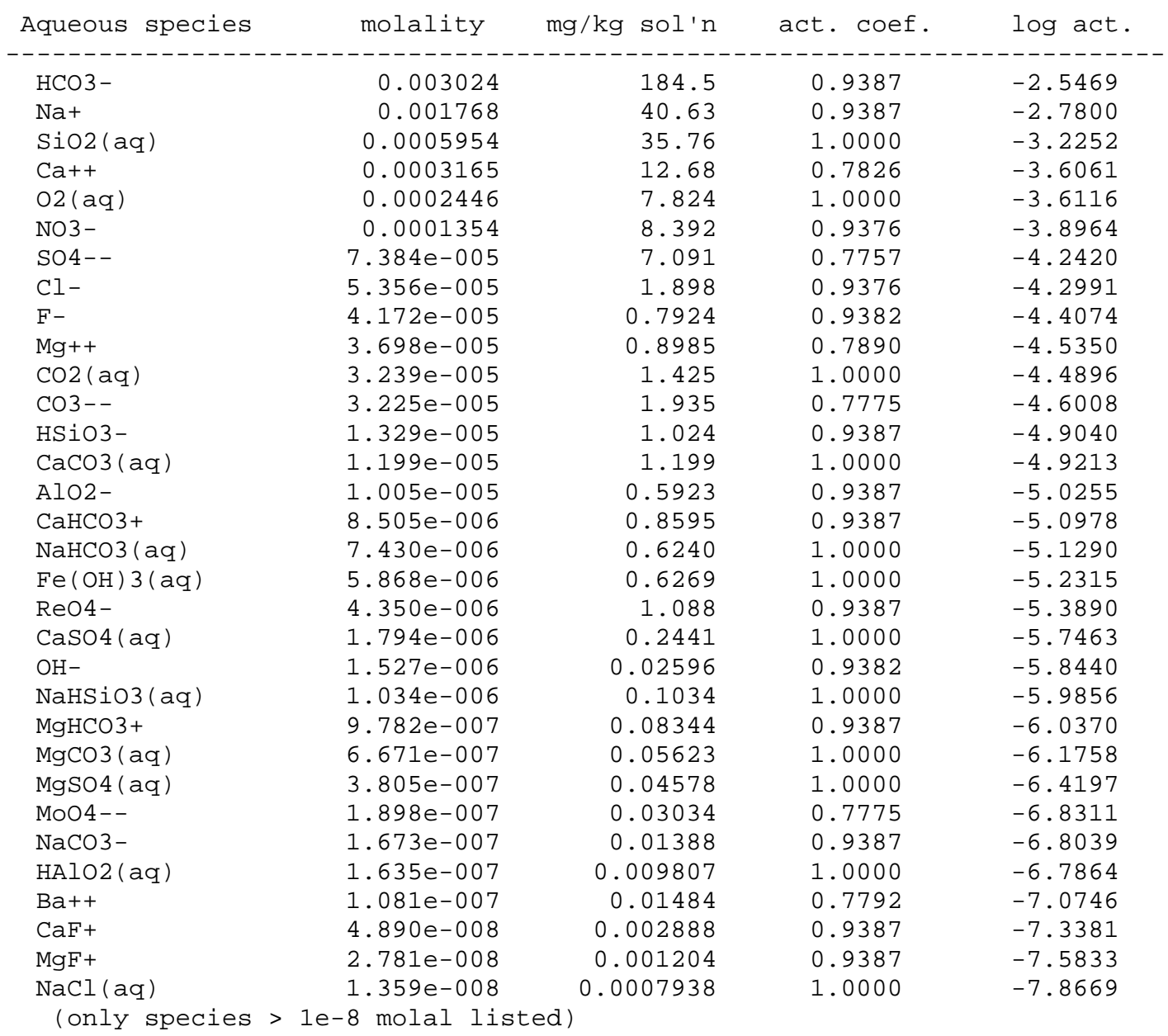




\begin{tabular}{|c|c|c|c|}
\hline lerad s & $\log \mathrm{Q} / \mathrm{K}$ & & $\log Q / K$ \\
\hline & & & - - - - - - - \\
\hline Nontronite-Ca & $18.7762 \mathrm{~s} / \mathrm{sat}$ & Clinochlore-14A & $2.1121 \mathrm{~s} / \mathrm{sat}$ \\
\hline Nontronite-Mg & $18.6516 \mathrm{~s} / \mathrm{sat}$ & Zoisite & $2.0815 \mathrm{~s} / \mathrm{sat}$ \\
\hline Nontronite-Na & $18.4179 \mathrm{~s} / \mathrm{sat}$ & Saponite-H & $2.0450 \mathrm{~s} / \mathrm{sat}$ \\
\hline Clinoptilolite-h & $17.9303 \mathrm{~s} / \mathrm{sat}$ & Talc & $2.0153 \mathrm{~s} / \mathrm{sat}$ \\
\hline Clinoptilolite-C & $17.9013 \mathrm{~s} / \mathrm{sat}$ & Gibbsite & $2.0147 \mathrm{~s} / \mathrm{sat}$ \\
\hline Nontronite-H & $17.6035 \mathrm{~s} / \mathrm{sat}$ & Albite_high & $1.7796 \mathrm{~s} / \mathrm{sat}$ \\
\hline Clinoptilolite-h & $14.8764 \mathrm{~s} / \mathrm{sat}$ & Witherite & $1.7594 \mathrm{~s} / \mathrm{sat}$ \\
\hline Clinoptilolite-N & $14.8755 \mathrm{~s} / \mathrm{sat}$ & Wairakite & $1.6471 \mathrm{~s} / \mathrm{sat}$ \\
\hline Hematite & $13.0455 \mathrm{~s} / \mathrm{sat}$ & Magnetite & $1.3084 \mathrm{~s} / \mathrm{sat}$ \\
\hline Epidote-ord & $9.3628 \mathrm{~s} / \mathrm{sat}$ & Corundum & $1.0743 \mathrm{~s} / \mathrm{sat}$ \\
\hline Epidote & $9.3623 \mathrm{~s} / \mathrm{sat}$ & Quartz & $0.9066 \mathrm{~s} / \mathrm{sat}$ \\
\hline Andradite & 8.8779s/sat & Dolomite & $0.7397 \mathrm{~s} / \mathrm{sat}$ \\
\hline Mesolite & $8.6153 \mathrm{~s} / \mathrm{sat}$ & Dolomite-ord & $0.7397 \mathrm{~s} / \mathrm{sat}$ \\
\hline Paragonite & $7.8597 \mathrm{~s} / \mathrm{sat}$ & Tridymite & $0.7090 \mathrm{~s} / \mathrm{sat}$ \\
\hline Beidellite-Ca & $7.8427 \mathrm{~s} / \mathrm{sat}$ & Jadeite & $0.6014 \mathrm{~s} / \mathrm{sat}$ \\
\hline Beidellite-Mg & $7.7180 \mathrm{~s} / \mathrm{sat}$ & Kyanite & $0.5962 \mathrm{~s} / \mathrm{sat}$ \\
\hline Beidellite-Na & $7.4843 \mathrm{~s} / \mathrm{sat}$ & Cristobalite(alp & $0.3446 \mathrm{~s} / \mathrm{sat}$ \\
\hline Montmor-Ca & $7.4215 \mathrm{~s} / \mathrm{sat}$ & Chalcedony & $0.3307 \mathrm{~s} / \mathrm{sat}$ \\
\hline Montmor-Mg & $7.3683 \mathrm{~s} / \mathrm{sat}$ & Andalusite & $0.3127 \mathrm{~s} / \mathrm{sat}$ \\
\hline Scolecite & $7.1380 \mathrm{~s} / \mathrm{sat}$ & Calcite & $0.2548 \mathrm{~s} / \mathrm{sat}$ \\
\hline Montmor-Na & $7.1310 \mathrm{~s} / \mathrm{sat}$ & Dawsonite & $0.1797 \mathrm{~s} / \mathrm{sat}$ \\
\hline Beidellite-H & $6.6700 \mathrm{~s} / \mathrm{sat}$ & Aragonite & $0.1102 \mathrm{~s} / \mathrm{sat}$ \\
\hline Pyrophyllite & $6.6433 \mathrm{~s} / \mathrm{sat}$ & Coesite & $0.0834 \mathrm{~s} / \mathrm{sat}$ \\
\hline Kaolinite & $6.5063 \mathrm{~s} / \mathrm{sat}$ & Sillimanite & $-\odot .0607$ \\
\hline Laumontite & $6.2176 \mathrm{~s} / \mathrm{sat}$ & Cristobalite (bet & -0.1134 \\
\hline Goethite & $6.0482 \mathrm{~s} / \mathrm{sat}$ & Ice & -0.1194 \\
\hline Amesite-14A & $4.5636 \mathrm{~s} / \mathrm{sat}$ & $\mathrm{Si02}(\mathrm{am})$ & -0.4215 \\
\hline Mordenite & $4.3496 \mathrm{~s} / \mathrm{sat}$ & Monohydrocalcite & -0.5660 \\
\hline Margarite & $4.3441 \mathrm{~s} / \mathrm{sat}$ & Anorthite & -0.6663 \\
\hline Ferrite-Ca & $4.2739 \mathrm{~s} / \mathrm{sat}$ & Dolomite-dis & -0.8421 \\
\hline Lawsonite & $3.8747 \mathrm{~s} / \mathrm{sat}$ & Analcime & -0.8767 \\
\hline Ferrite-Mg & $3.7673 \mathrm{~s} / \mathrm{sat}$ & Magnesite & -1.1751 \\
\hline Saponite-Ca & $3.2177 \mathrm{~s} / \mathrm{sat}$ & Barite & -1.2501 \\
\hline Albite_low & $3.1319 \mathrm{~s} / \mathrm{sat}$ & Tremolite & -1.2803 \\
\hline Albite & $3.1318 \mathrm{~s} / \mathrm{sat}$ & Clinochlore-7A & -1.3029 \\
\hline Saponite-Mg & $3.0937 \mathrm{~s} / \mathrm{sat}$ & Nepheline & -1.7715 \\
\hline Saponite-Na & $2.8594 \mathrm{~s} / \mathrm{sat}$ & Chrysotile & -1.7985 \\
\hline Prehnite & $2.8148 \mathrm{~s} / \mathrm{sat}$ & Fluorite & -2.3338 \\
\hline Natrolite & $2.5957 \mathrm{~s} / \mathrm{sat}$ & Diopside & -2.5780 \\
\hline Diaspore & $2.5866 \mathrm{~s} / \mathrm{sat}$ & $\mathrm{Fe}(\mathrm{OH}) 3$ & -2.6397 \\
\hline Boehmite & $2.1742 \mathrm{~s} / \mathrm{sat}$ & Enstatite & -2.6458 \\
\hline Clinozoisite & $2.1274 \mathrm{~s} / \mathrm{sat}$ & & \\
\hline (only minerals & with $\log Q / K>$ & 3 listed) & \\
\hline Gases & fugacity & $\log$ fug. & \\
\hline 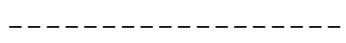 & $\ldots$ & - - - - - - & \\
\hline $02(g)$ & 0.1745 & -0.758 & \\
\hline $\mathrm{H} 2 \mathrm{O}(\mathrm{g})$ & 0.01903 & -1.721 & \\
\hline $\operatorname{co2}(\mathrm{g})$ & ๑. 0008099 & -3.092 & \\
\hline$H F(g)$ & $9.256 e-015$ & -14.034 & \\
\hline $\mathrm{HCl}(\mathrm{g})$ & $6.972 \mathrm{e}-020$ & -19.157 & \\
\hline N02 $(\mathrm{g})$ & $1.200 \mathrm{e}-020$ & -19.921 & \\
\hline $\mathrm{N} 2(\mathrm{~g})$ & $5.703 e-021$ & -20.244 & \\
\hline NO $(g)$ & $1.204 \mathrm{e}-026$ & -25.919 & \\
\hline $\operatorname{cl} 2(\mathrm{~g})$ & $5.970 \mathrm{e}-031$ & -30.224 & \\
\hline $\operatorname{SiF} 4(g)$ & $8.815 e-040$ & -39.055 & \\
\hline $\mathrm{H} 2(\mathrm{~g})$ & $8.972 \mathrm{e}-043$ & -42.047 & \\
\hline $\operatorname{co}(\mathrm{g})$ & $2.322 \mathrm{e}-049$ & -48.634 & \\
\hline $\mathrm{S} 02(\mathrm{~g})$ & $1.636 \mathrm{e}-058$ & -57.786 & \\
\hline $\mathrm{NH3}(\mathrm{g})$ & $6.757 e-071$ & -70.170 & \\
\hline
\end{tabular}




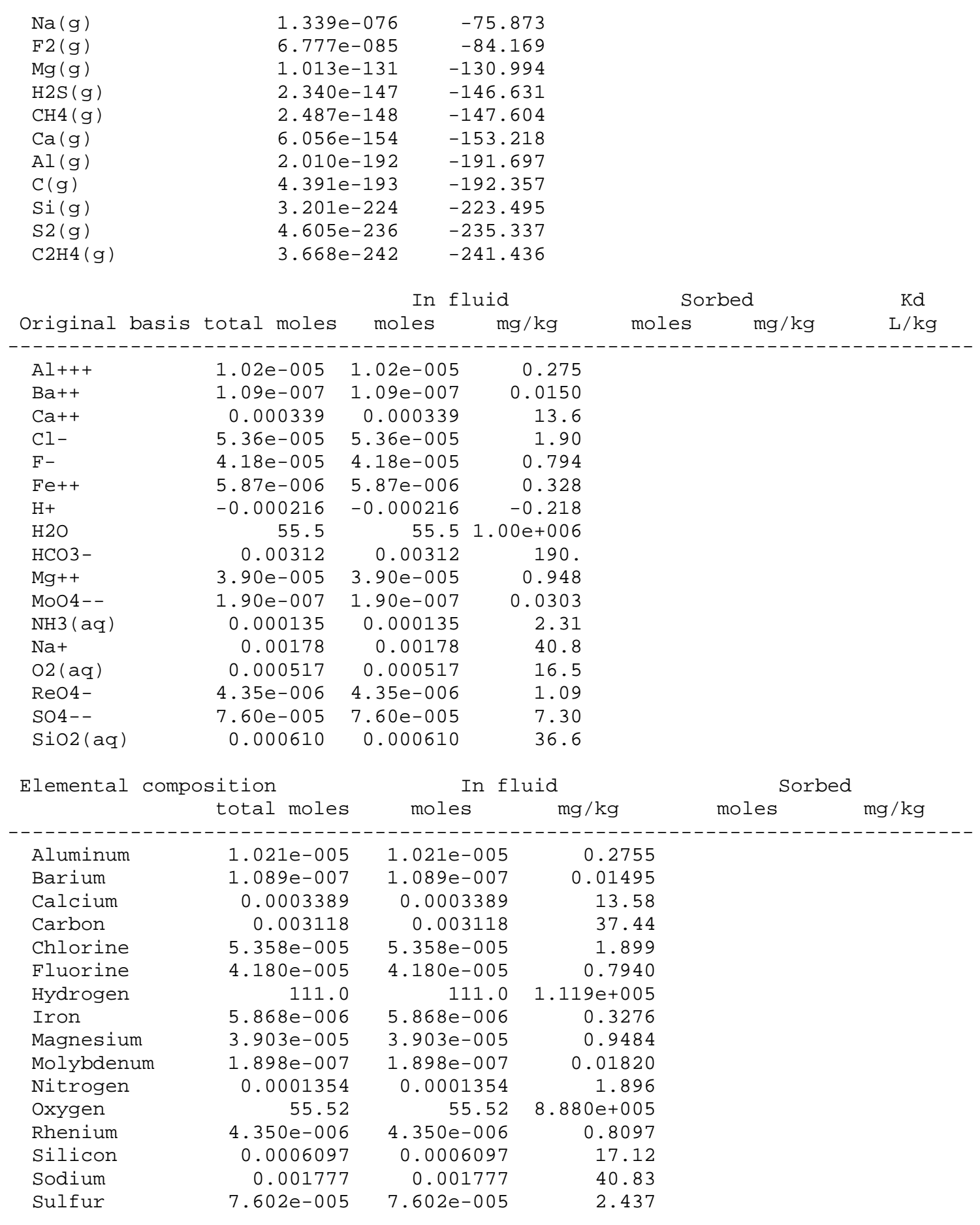




\section{Sample D14NEB0T}

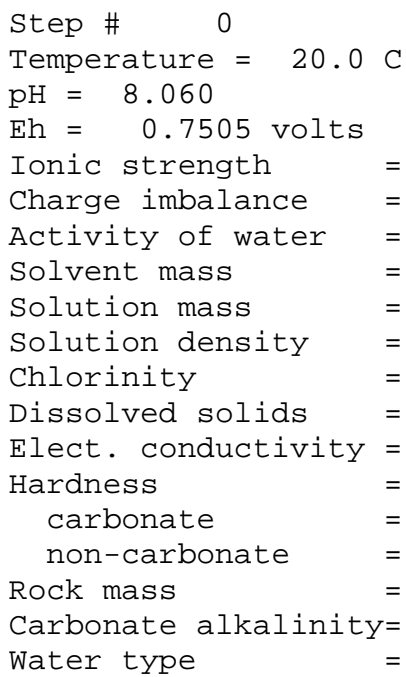

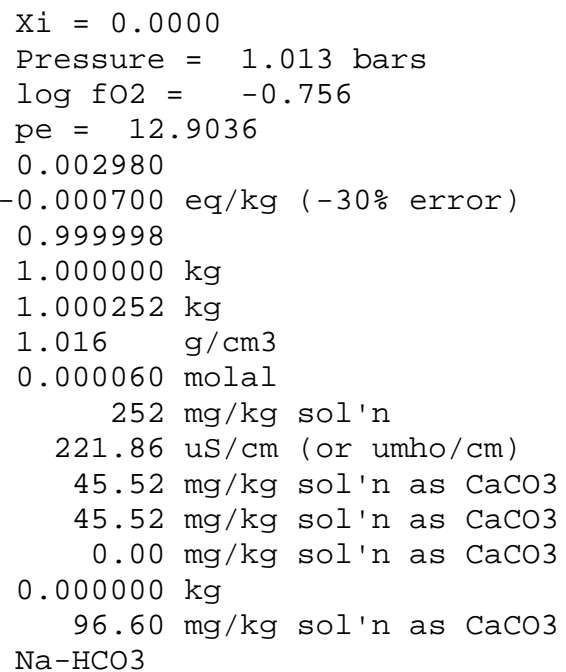

No minerals in system.

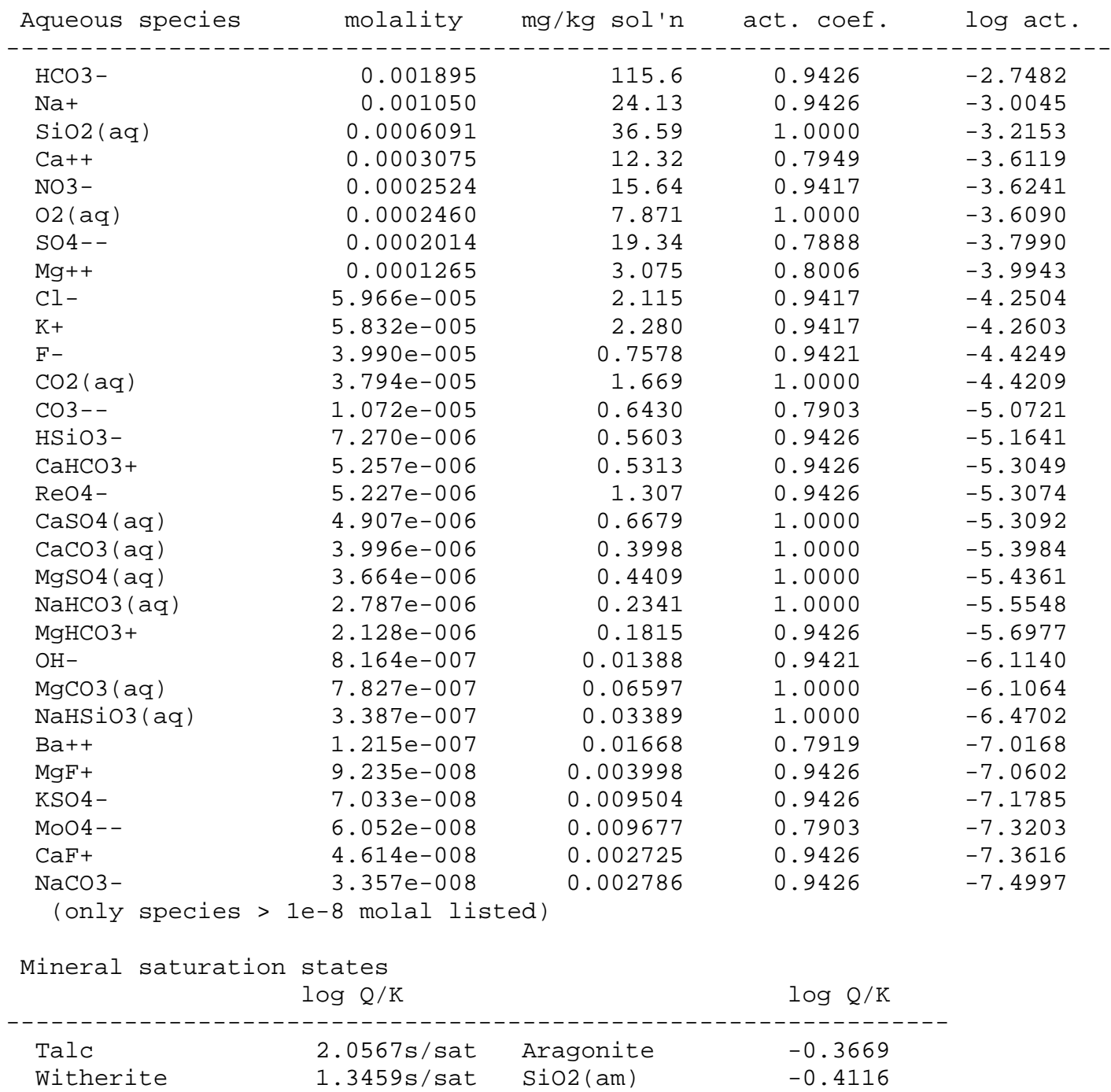




\begin{tabular}{|c|c|c|c|c|c|}
\hline Quartz & \multicolumn{2}{|c|}{$\odot .9165 \mathrm{~s} / \mathrm{sat}$} & Barite & \multicolumn{2}{|l|}{-0.7494} \\
\hline Tridymite & \multicolumn{2}{|c|}{$0.7189 \mathrm{~s} / \mathrm{sat}$} & Monohydrocalcite & -1.0431 & \\
\hline Cristobalite(alp & \multicolumn{2}{|c|}{$\odot .3545 \mathrm{~s} / \mathrm{sat}$} & Magnesite & -1.1057 & \\
\hline Chalcedony & \multicolumn{2}{|c|}{$0.3406 \mathrm{~s} / \mathrm{sat}$} & Dolomite-dis & -1.2498 & \\
\hline Dolomite & \multicolumn{2}{|c|}{$0.3320 \mathrm{~s} / \mathrm{sat}$} & Chrysotile & -1.7768 & \\
\hline Dolomite-ord & \multicolumn{2}{|c|}{$0.3320 \mathrm{~s} / \mathrm{sat}$} & Tremolite & -2.2898 & \\
\hline Coesite & \multicolumn{2}{|c|}{$0.0933 \mathrm{~s} / \mathrm{sat}$} & Fluorite & -2.3748 & \\
\hline Cristobalite (bet & \multicolumn{2}{|c|}{-0.1035} & Enstatite & -2.6353 & \\
\hline Ice & -0.1194 & & Gypsum & -2.9205 & \\
\hline $\begin{array}{l}\text { Calcite } \\
\text { (only minerals }\end{array}$ & $\begin{array}{l}-0.2223 \\
\text { with } \log \end{array}$ & $Q / K>-3$ & listed) & & \\
\hline Gases & fugac & ity & $\log$ fug. & & \\
\hline 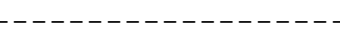 & 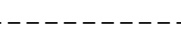 & $-\ldots-\ldots$ & 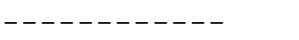 & & \\
\hline $02(g)$ & & .1755 & -0.756 & & \\
\hline $\mathrm{H} 2 \mathrm{O}(\mathrm{g})$ & $\odot$. & 01903 & -1.721 & & \\
\hline $\mathrm{co2}(\mathrm{g})$ & 0.00 & 09488 & -3.023 & & \\
\hline $\operatorname{HF}(g)$ & 1.655 & -014 & -13.781 & & \\
\hline $\mathrm{HCl}(\mathrm{g})$ & $1.452 \epsilon$ & -019 & -18.838 & & \\
\hline $\mathrm{N} 2(\mathrm{~g})$ & 6.827 & -020 & -19.166 & & \\
\hline N02 $(g)$ & 4.178 & -020 & -19.379 & & \\
\hline $\mathrm{NO}(\mathrm{g})$ & 4.178 & -026 & -25.379 & & \\
\hline Cl2(g) & 2.599 & $2-030$ & -29.585 & & \\
\hline $\operatorname{SiF} 4(g)$ & 9.223 & -039 & -38.035 & & \\
\hline $\mathrm{H} 2(\mathrm{~g})$ & 8.945 & -043 & -42.048 & & \\
\hline $\operatorname{co}(g)$ & 2.712 & -049 & -48.567 & & \\
\hline so2(g) & 1.568 & -057 & -56.805 & & \\
\hline $\mathrm{NH} 3(\mathrm{~g})$ & 2.327 & -070 & -69.633 & & \\
\hline $\mathrm{Na}(\mathrm{g})$ & 4.281 & -077 & -76.368 & & \\
\hline $\mathrm{K}(\mathrm{g})$ & 4.768 & -079 & -78.322 & & \\
\hline $\mathrm{F} 2(\mathrm{~g})$ & $2.174 \epsilon$ & -084 & -83.663 & & \\
\hline $\operatorname{Mg}(g)$ & 1.012 & -131 & -130.995 & & \\
\hline $\mathrm{H} 2 \mathrm{~S}(\mathrm{~g})$ & 2.223 & -146 & -145.653 & & \\
\hline $\mathrm{CH} 4(\mathrm{~g})$ & 2.879 & -148 & -147.541 & & \\
\hline $\mathrm{Ca}(\mathrm{g})$ & 1.718 & -154 & -153.765 & & \\
\hline$C(g)$ & $5.114 \epsilon$ & -193 & -192.291 & & \\
\hline $\operatorname{si}(g)$ & 3.256 & -224 & -223.487 & & \\
\hline $\mathrm{S} 2(\mathrm{~g})$ & $4.182 \epsilon$ & -234 & -233.379 & & \\
\hline $\mathrm{C} 2 \mathrm{H} 4(\mathrm{~g})$ & 4.944 & -242 & -241.306 & & \\
\hline & & In & fluid & Sorbed & $\mathrm{Kd}$ \\
\hline Original basis tot & tal moles & moles & $\mathrm{mg} / \mathrm{kg}$ & moles $\quad \mathrm{mg} / \mathrm{kg}$ & $\mathrm{L} / \mathrm{kg}$ \\
\hline 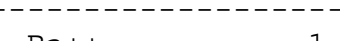 & -----1 & & & & \\
\hline $\mathrm{Ba}++$ & $.22 \mathrm{e}-007$ & $1.22 \mathrm{e}-\odot \odot$ & 0.0167 & & \\
\hline $\mathrm{Ca}++$ & $\odot .00 \odot 322$ & $\odot .0 \odot \odot 32$ & 12.9 & & \\
\hline $\mathrm{Cl}-$ & $.97 e-005$ & $5.97 \mathrm{e}-\odot \odot$ & 2.12 & & \\
\hline $\mathrm{F}-$ & $.00 \mathrm{e}-0 \odot 5$ & 4. $\odot \odot \mathrm{e}-\odot \odot$ & 0.761 & & \\
\hline $\mathrm{H}+$ & $\odot .000238$ & $-\odot .0 \odot \odot 23$ & -0.240 & & \\
\hline $\mathrm{H} 2 \mathrm{O}$ & 55.5 & 55 & $.51 . \odot \odot \mathrm{e}+\odot \odot 6$ & & \\
\hline HCO3 - & $\odot .00196$ & ๑. 0019 & $96 \quad 119$ & & \\
\hline $\mathrm{K}+$ & $.84 e-005$ & $5.84 \mathrm{e}-\odot \odot$ & 2.28 & & \\
\hline$M g++$ & $\odot .000133$ & $\odot .0 \odot \odot 13$ & 3.24 & & \\
\hline Mo04 - - & $.05 e-008$ & $6.05 e-0 \odot$ & $\odot .0 \odot 968$ & & \\
\hline $\mathrm{NH} 3(\mathrm{aq})$ & $\odot .0 \odot \odot 252$ & $\odot .0 \odot \odot 25$ & 4.30 & & \\
\hline $\mathrm{Na}+$ & $\odot .00105$ & 0.0010 & 24.2 & & \\
\hline $02(a q)$ & $\odot .000751$ & $\odot .00075$ & 24.0 & & \\
\hline Re04- & $.23 e-0 \odot 6$ & $5.23 e-\odot \odot$ & 1.31 & & \\
\hline SO4 - - & $\odot .000210$ & 0.00021 & 20.2 & & \\
\hline Si02 (aq) & $\odot .0 \odot 0617$ & 0.00061 & 37.0 & & \\
\hline
\end{tabular}




\begin{tabular}{|c|c|c|c|c|c|}
\hline \multicolumn{2}{|c|}{ Elemental composition } & \multicolumn{2}{|c|}{ In fluid } & \multicolumn{2}{|c|}{ Sorbed } \\
\hline & total moles & moles & $\mathrm{mg} / \mathrm{kg}$ & moles & $\mathrm{mg} / \mathrm{kg}$ \\
\hline$-\ldots$ & - - - - - - - - - - & - - - - - - - - - & 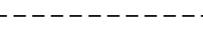 & ---- & \\
\hline Barium & 1. $218 e-\odot \odot 7$ & $1.218 \mathrm{e}-0 \odot 7$ & 0.01673 & & \\
\hline Calcium & 0.0003217 & 0.0003217 & 12.89 & & \\
\hline Carbon & ๑. . 001958 & ๑. . 001958 & 23.51 & & \\
\hline Chlorine & $5.968 \mathrm{e}-005$ & $5.968 e-005$ & 2.115 & & \\
\hline Fluorine & $4.004 \mathrm{e}-005$ & $4.004 \mathrm{e}-005$ & 0.7605 & & \\
\hline Hydrogen & 111.0 & 111.0 & $1.119 \mathrm{e}+005$ & & \\
\hline Magnesium & 0.0001332 & 0.0001332 & 3.237 & & \\
\hline Molybdenum & $6.052 \mathrm{e}-008$ & $6.052 e-008$ & $\odot .005805$ & & \\
\hline Nitrogen & $\odot .0 \odot \odot 2524$ & $\odot .0 \odot \odot 2524$ & 3.534 & & \\
\hline oxygen & 55.52 & 55.52 & $8.880 e+005$ & & \\
\hline Potassium & $5.840 \mathrm{e}-\odot \odot 5$ & $5.840 \mathrm{e}-005$ & 2.283 & & \\
\hline Rhenium & $5.227 e-0 \odot 6$ & $5.227 e-0 \odot 6$ & 0.9730 & & \\
\hline Silicon & $\odot .0 \odot \odot 6167$ & $\odot . \odot \odot \odot 6167$ & 17.32 & & \\
\hline Sodium & ๑. .001053 & 0.001053 & 24.20 & & \\
\hline Sulfur & 0.0002100 & 0.0002100 & 6.733 & & \\
\hline
\end{tabular}




\section{Sample D14NWB0T}

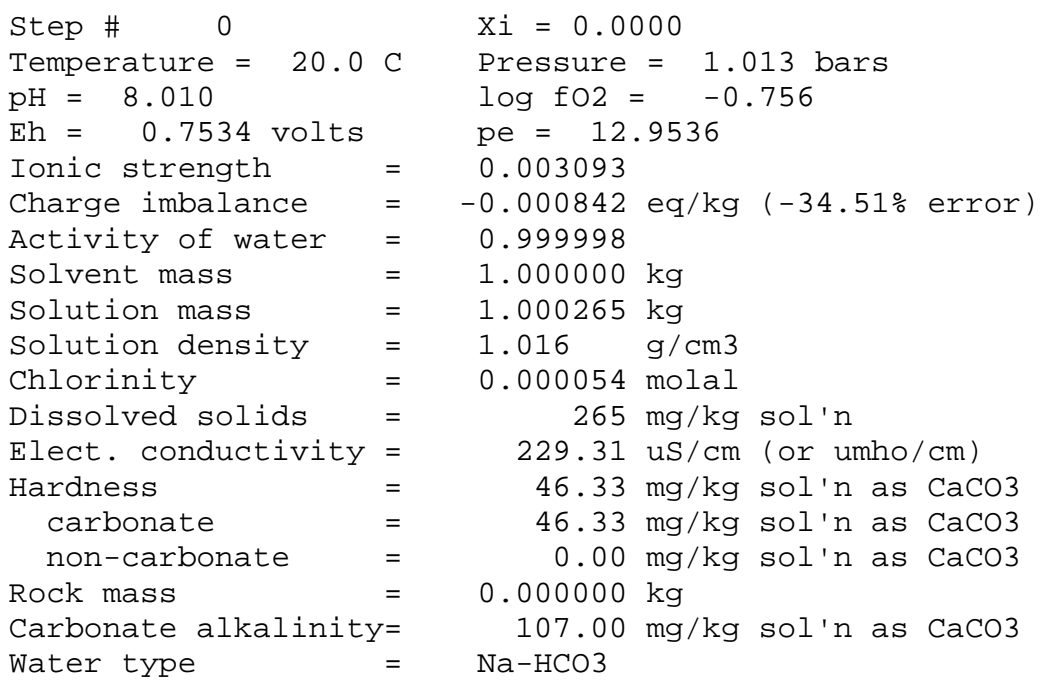

No minerals in system.

\begin{tabular}{|c|c|c|c|c|}
\hline Aqueous species & molality & $\mathrm{mg} / \mathrm{kg}$ sol'n & act. coef. & $\log$ act \\
\hline - - - - - - - - - - - & $-8-2-1$ & 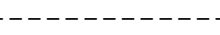 & ----- & ---- \\
\hline HCO3 - & $\odot .0 \odot 2101$ & 128.2 & $\odot .9416$ & -2.7036 \\
\hline $\mathrm{Na}+$ & $\odot .001067$ & 24.52 & 0.9416 & -2.9981 \\
\hline $\operatorname{Si02}(\mathrm{aq})$ & ๑. . 0006238 & 37.47 & 1.0000 & -3.2050 \\
\hline $\mathrm{Ca}++$ & 0.0003117 & 12.49 & 0.7918 & -3.6076 \\
\hline $02(\mathrm{aq})$ & 0.0002460 & 7.871 & 1.0000 & -3.6090 \\
\hline N03- & $\odot .0 \odot \odot 227 \odot$ & 14.07 & 0.9407 & -3.6706 \\
\hline SO4 - - & ๑. . $0 \odot \odot 2013$ & 19.33 & $\odot .7855$ & -3.8010 \\
\hline $\mathrm{Mg}++$ & 0.0001294 & 3.145 & 0.7977 & -3.9861 \\
\hline $\mathrm{K}+$ & $6.109 \mathrm{e}-\odot \odot 5$ & 2.388 & $\odot .9407$ & -4.2406 \\
\hline $\mathrm{Cl}-$ & $5.384 e-005$ & 1.908 & 0.9407 & -4.2955 \\
\hline $\mathrm{co2}(\mathrm{aq})$ & $4.717 e-005$ & 2.075 & 1.0000 & -4.3264 \\
\hline $\mathrm{F}-$ & $4.284 \mathrm{e}-\odot \odot 5$ & $\odot .8137$ & $\odot .9412$ & -4.3945 \\
\hline CO3-- & $1.063 e-005$ & 0.6376 & $\odot .7871$ & -5.0775 \\
\hline HSi03- & $6.642 \mathrm{e}-006$ & 0.5119 & 0.9416 & -5.2038 \\
\hline CaHCO3+ & $5.889 e-\odot \odot 6$ & $\odot .5952$ & $\odot .9416$ & -5.2561 \\
\hline CaSo4(aq) & $4.933 e-006$ & 0.6714 & 1.0000 & -5.3069 \\
\hline Re04 - & $4.202 e-006$ & 1.051 & 0.9416 & -5.4027 \\
\hline CaC03( aq) & $3.986 \mathrm{e}-0 \odot 6$ & 0.3988 & 1.0000 & -5.3995 \\
\hline $\operatorname{MgSO} 4(\mathrm{aq})$ & $3.717 \mathrm{e}-0 \odot 6$ & 0.4473 & 1.0000 & -5.4298 \\
\hline $\operatorname{NaHCO} 3(\mathrm{aq})$ & $3.135 \mathrm{e}-006$ & 0.2633 & 1.0000 & -5.5038 \\
\hline $\mathrm{MgHCO} 3+$ & $2.406 \mathrm{e}-006$ & 0.2052 & 0.9416 & -5.6449 \\
\hline $\operatorname{MgC03}(\mathrm{aq})$ & $7.877 e-007$ & ๑. .06640 & 1.0000 & -6.1036 \\
\hline $\mathrm{OH}-$ & $7.284 \mathrm{e}-0 \odot 7$ & 0.01238 & $\odot .9412$ & -6.1640 \\
\hline NaHSiO3(aq) & $3.138 \mathrm{e}-007$ & 0.03139 & 1.0000 & -6.5034 \\
\hline $\mathrm{Ba}++$ & $1.158 e-007$ & 0.01590 & 0.7887 & -7.0394 \\
\hline MgF+ & 1. $011 \mathrm{e}-007$ & $\odot .004375$ & 0.9416 & -7.0215 \\
\hline Mo04 - - & $7.591 \mathrm{e}-0 \odot 8$ & 0.01214 & 0.7871 & -7.2237 \\
\hline KSO4 - & $7.333 e-0 \odot 8$ & ๑.009909 & $\odot .9416$ & -7.1608 \\
\hline $\mathrm{CaF}+$ & $5.0 \odot 4 e-0 \odot 8$ & ๑.002955 & 0.9416 & -7.3268 \\
\hline $\mathrm{NaC03-}$ & $3.369 e-008$ & ๑. 002795 & 0.9416 & -7.4987 \\
\hline $\mathrm{H}+$ & 1. $033 e-008$ & $1.041 \mathrm{e}-0 \odot 5$ & 0.9461 & -8.0100 \\
\hline
\end{tabular}




\begin{tabular}{|c|c|c|c|}
\hline Mineral saturatio & $\log Q / K$ & & $\log Q / K$ \\
\hline Talc & $1.8228 \mathrm{~s} / \mathrm{sat}$ & Aragonite & -0.3680 \\
\hline Witherite & $1.3178 \mathrm{~s} / \mathrm{sat}$ & Si02(am) & -0.4013 \\
\hline Quartz & $\odot .9268 \mathrm{~s} / \mathrm{sat}$ & Barite & -0.7740 \\
\hline Tridymite & $0.7292 \mathrm{~s} / \mathrm{sat}$ & Monohydrocalcite & -1.0442 \\
\hline Cristobalite(alp & $0.3648 \mathrm{~s} / \mathrm{sat}$ & Magnesite & -1.1029 \\
\hline Chalcedony & $\odot .3509 \mathrm{~s} / \mathrm{sat}$ & Dolomite-dis & -1.2482 \\
\hline Dolomite & $\odot .3336 \mathrm{~s} / \mathrm{sat}$ & Chrysotile & -2.0314 \\
\hline Dolomite-ord & $\odot .3336 \mathrm{~s} / \mathrm{sat}$ & Fluorite & -2.3097 \\
\hline Coesite & $0.1036 \mathrm{~s} / \mathrm{sat}$ & Enstatite & -2.7167 \\
\hline Cristobalite (bet & -0.0932 & Tremolite & -2.8573 \\
\hline Ice & -0.1194 & Gypsum & -2.9182 \\
\hline Calcite & -0.2235 & & \\
\hline (only minerals & with $\log Q / K>$ & 3 listed) & \\
\hline Gases & fugacity & $\log$ fug. & \\
\hline 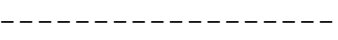 & $---\cdot--\cdot-$ & - & \\
\hline $02(g)$ & 0.1755 & -0.756 & \\
\hline $\mathrm{H} 2 \mathrm{O}(\mathrm{g})$ & 0.01903 & -1.721 & \\
\hline $\operatorname{co2}(\mathrm{g})$ & 0.001180 & -2.928 & \\
\hline $\mathrm{HF}(\mathrm{g})$ & 1. $992 \mathrm{e}-014$ & -13.701 & \\
\hline $\mathrm{HCl}(\mathrm{g})$ & $1.469 e-019$ & -18.833 & \\
\hline $\mathrm{N} 2(\mathrm{~g})$ & $6.937 \mathrm{e}-02 \odot$ & -19.159 & \\
\hline N02(g) & $4.212 e-020$ & -19.376 & \\
\hline NO $(g)$ & $4.211 e-026$ & -25.376 & \\
\hline $\operatorname{cl} 2(\mathrm{~g})$ & $2.658 \mathrm{e}-030$ & -29.575 & \\
\hline $\operatorname{SiF} 4(g)$ & $1.981 \mathrm{e}-038$ & -37.703 & \\
\hline $\mathrm{H} 2(\mathrm{~g})$ & $8.945 e-043$ & -42.048 & \\
\hline $\mathrm{co}(\mathrm{g})$ & $3.371 \mathrm{e}-049$ & -48.472 & \\
\hline $\mathrm{SO} 2(\mathrm{~g})$ & $1.965 e-057$ & -56.707 & \\
\hline $\mathrm{NH3}(\mathrm{g})$ & $2.346 \mathrm{e}-070$ & -69.630 & \\
\hline $\mathrm{Na}(\mathrm{g})$ & $3.873 e-077$ & -76.412 & \\
\hline$K(g)$ & $4.446 e-079$ & -78.352 & \\
\hline $\mathrm{F} 2(\mathrm{~g})$ & $3.148 e-084$ & -83.502 & \\
\hline $\operatorname{Mg}(g)$ & $8.189 e-132$ & -131.087 & \\
\hline $\mathrm{H} 2 \mathrm{~S}(\mathrm{~g})$ & $2.786 e-146$ & -145.555 & \\
\hline $\mathrm{CH} 4(\mathrm{~g})$ & $3.579 e-148$ & -147.446 & \\
\hline $\mathrm{Ca}(\mathrm{g})$ & $1.378 e-154$ & -153.861 & \\
\hline$C(g)$ & $6.358 e-193$ & -192.197 & \\
\hline Si(g) & $3.334 \mathrm{e}-224$ & -223.477 & \\
\hline $\mathrm{S} 2(\mathrm{~g})$ & $6.568 e-234$ & -233.183 & \\
\hline $\mathrm{C} 2 \mathrm{H} 4(\mathrm{~g})$ & $7.643 e-242$ & -241.117 & \\
\hline
\end{tabular}




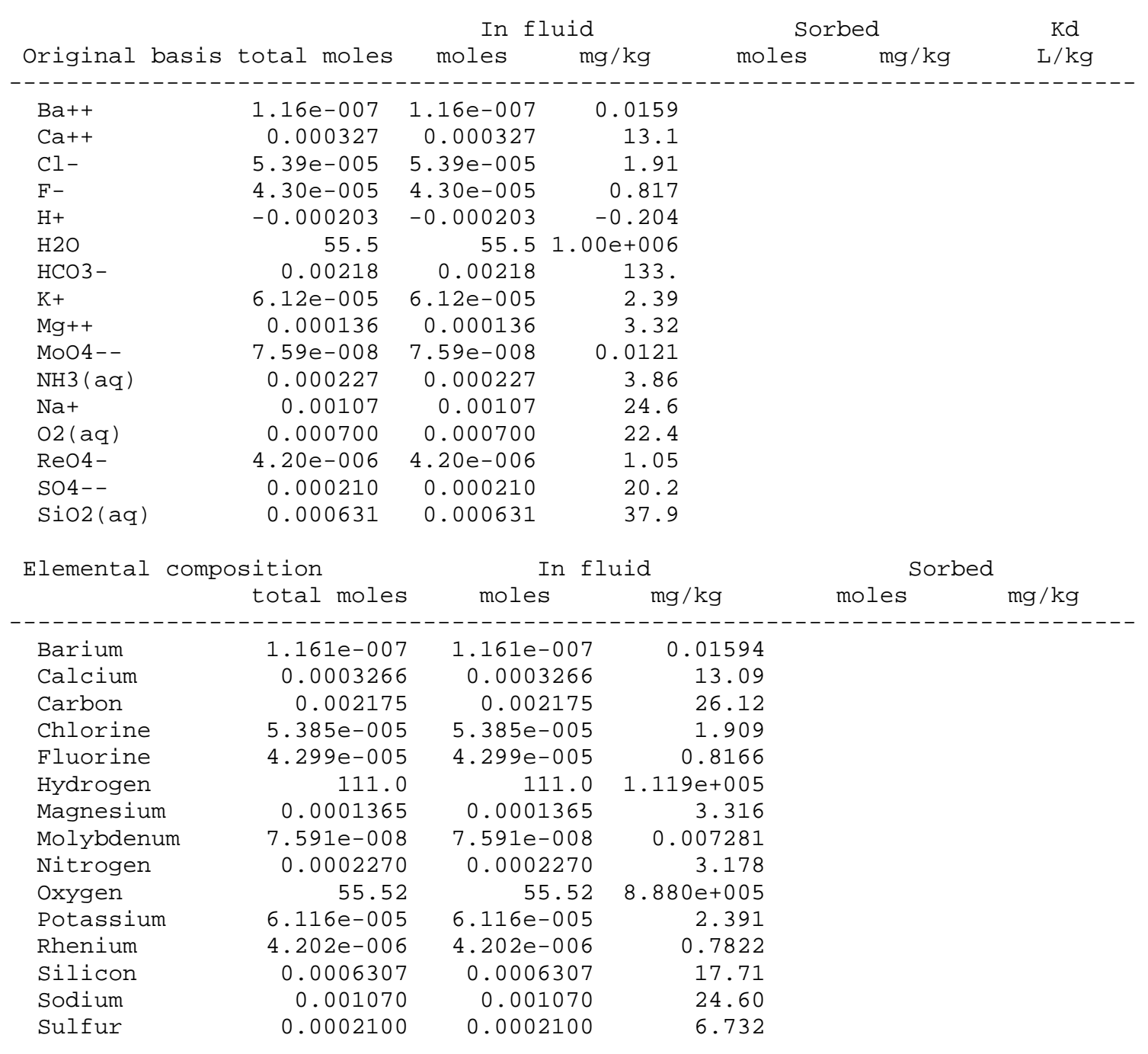




\section{Sample D14SEB0T}

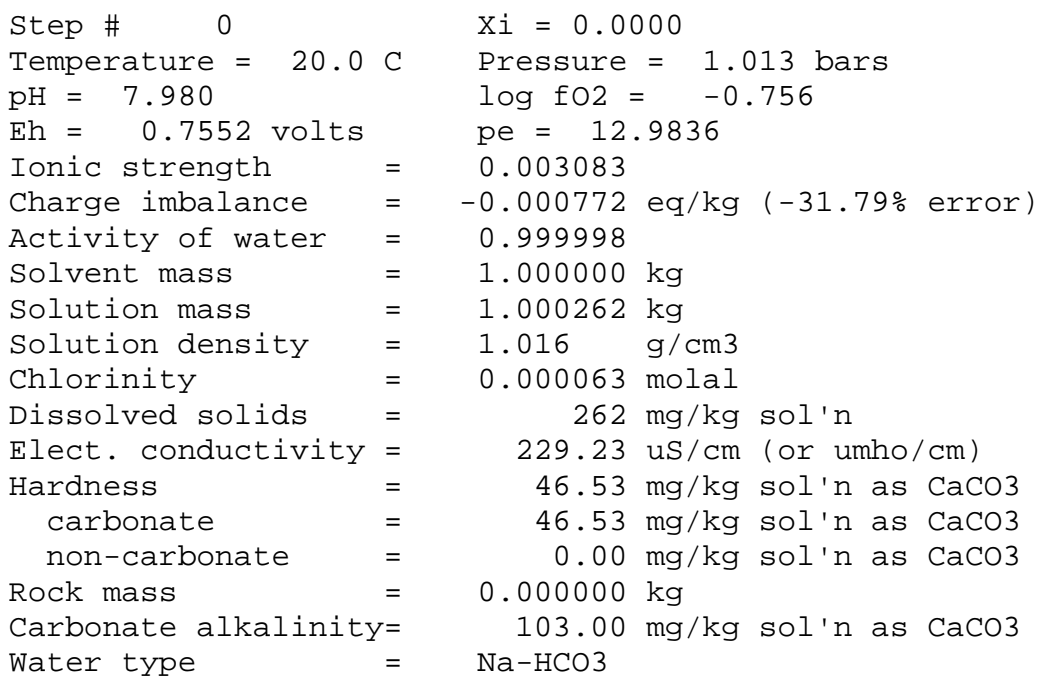

No minerals in system.

\begin{tabular}{|c|c|c|c|c|}
\hline Aqueous species & molality & $\mathrm{mg} / \mathrm{kg}$ sol'n & act. coef. & $\log$ act \\
\hline---------- & - - - - - - - - & - - - - - - - - - & - - - - - - - - & ---- \\
\hline $\mathrm{HCO} 3-$ & $\odot .0 \odot 2024$ & 123.5 & $\odot .9417$ & -2.7198 \\
\hline $\mathrm{Na}+$ & 0.001075 & 24.72 & $\odot .9417$ & -2.9945 \\
\hline $\operatorname{SiO} 2(\mathrm{aq})$ & 0.0006069 & 36.45 & 1.0000 & -3.2169 \\
\hline $\mathrm{Ca}++$ & ๑. . $00 \odot 3101$ & 12.42 & 0.7921 & -3.6098 \\
\hline N03 - & 0.0002619 & 16.23 & 0.9407 & -3.6084 \\
\hline $02(\mathrm{aq})$ & $\odot .0 \odot \odot 2460$ & 7.871 & $1.000 \odot$ & -3.6090 \\
\hline SO4 - - & $\odot .0001962$ & 18.84 & 0.7858 & -3.8120 \\
\hline $\mathrm{Mg}++$ & 0.0001339 & 3.254 & 0.7980 & -3.9712 \\
\hline $\mathrm{K}+$ & $6.587 e-0 \odot 5$ & 2.575 & 0.9407 & -4.2078 \\
\hline $\mathrm{Cl}-$ & $6.272 \mathrm{e}-0 \odot 5$ & 2.223 & 0.9407 & -4.2291 \\
\hline $\operatorname{co2}(\mathrm{aq})$ & $4.869 e-005$ & 2.142 & 1.0000 & -4.3125 \\
\hline $\mathrm{F}-$ & $4.325 e-0 \odot 5$ & $\odot .8214$ & $\odot .9412$ & -4.3903 \\
\hline CO3-- & $9.552 \mathrm{e}-006$ & 0.5731 & 0.7874 & -5.1237 \\
\hline HSi03 - & $6.031 e-006$ & 0.4648 & 0.9417 & -5.2457 \\
\hline $\mathrm{CaHCO}+$ & $5.645 e-006$ & 0.5706 & 0.9417 & -5.2744 \\
\hline Re04- & $5.497 e-006$ & 1.375 & 0.9417 & -5.2860 \\
\hline CaSO4(aq) & $4.787 e-0 \odot 6$ & $\odot .6515$ & 1.0000 & -5.3200 \\
\hline $\operatorname{MgSO} 4(\mathrm{aq})$ & $3.751 \mathrm{e}-006$ & 0.4514 & 1.0000 & -5.4259 \\
\hline $\mathrm{CaC0} 3(\mathrm{aq})$ & $3.566 e-006$ & 0.3568 & 1.0000 & -5.4478 \\
\hline $\mathrm{Zn++}$ & $3.046 e-0 \odot 6$ & $\odot .1991$ & $\odot .7921$ & -5.6175 \\
\hline $\mathrm{NaHCO} 3(\mathrm{aq})$ & $3.045 \mathrm{e}-006$ & 0.2558 & 1.0000 & -5.5164 \\
\hline $\mathrm{MgHCO} 3+$ & $2.399 e-0 \odot 6$ & 0.2046 & 0.9417 & -5.6461 \\
\hline $\operatorname{MgC0} 3(a q)$ & $7.330 \mathrm{e}-007$ & 0.06179 & 1.0000 & -6.1349 \\
\hline $\mathrm{OH}-$ & $6.797 \mathrm{e}-0 \odot 7$ & ๑. 01156 & 0.9412 & -6.1940 \\
\hline NaHSiO3 (aq) & $2.872 \mathrm{e}-0 \odot 7$ & 0.02874 & 1.0000 & -6.5418 \\
\hline $\mathrm{Ba}++$ & $1.137 \mathrm{e}-007$ & 0.01561 & 0.7890 & -7.0473 \\
\hline Mo04 - - & $1.057 \mathrm{e}-0 \odot 7$ & ๑. . 01689 & 0.7874 & -7.0799 \\
\hline $\mathrm{MgF}+$ & 1. $056 \mathrm{e}-007$ & 0.004571 & 0.9417 & -7.0025 \\
\hline KSO4 - & $7.710 \mathrm{e}-0 \odot 8$ & 0.01042 & 0.9417 & -7.1390 \\
\hline ZnSO4(aq) & $6.886 e-0 \odot 8$ & 0.01111 & 1.0000 & -7.1620 \\
\hline $\mathrm{CaF}+$ & $5.026 e-0 \odot 8$ & $\odot .0 \odot 2969$ & $\odot .9417$ & -7.3248 \\
\hline NaC03 - & $3.054 \mathrm{e}-008$ & ๑. .002534 & 0.9417 & -7.5413 \\
\hline $\mathrm{H}+$ & $1.107 \mathrm{e}-0 \odot 8$ & $1.115 \mathrm{e}-005$ & 0.9462 & -7.9800 \\
\hline
\end{tabular}




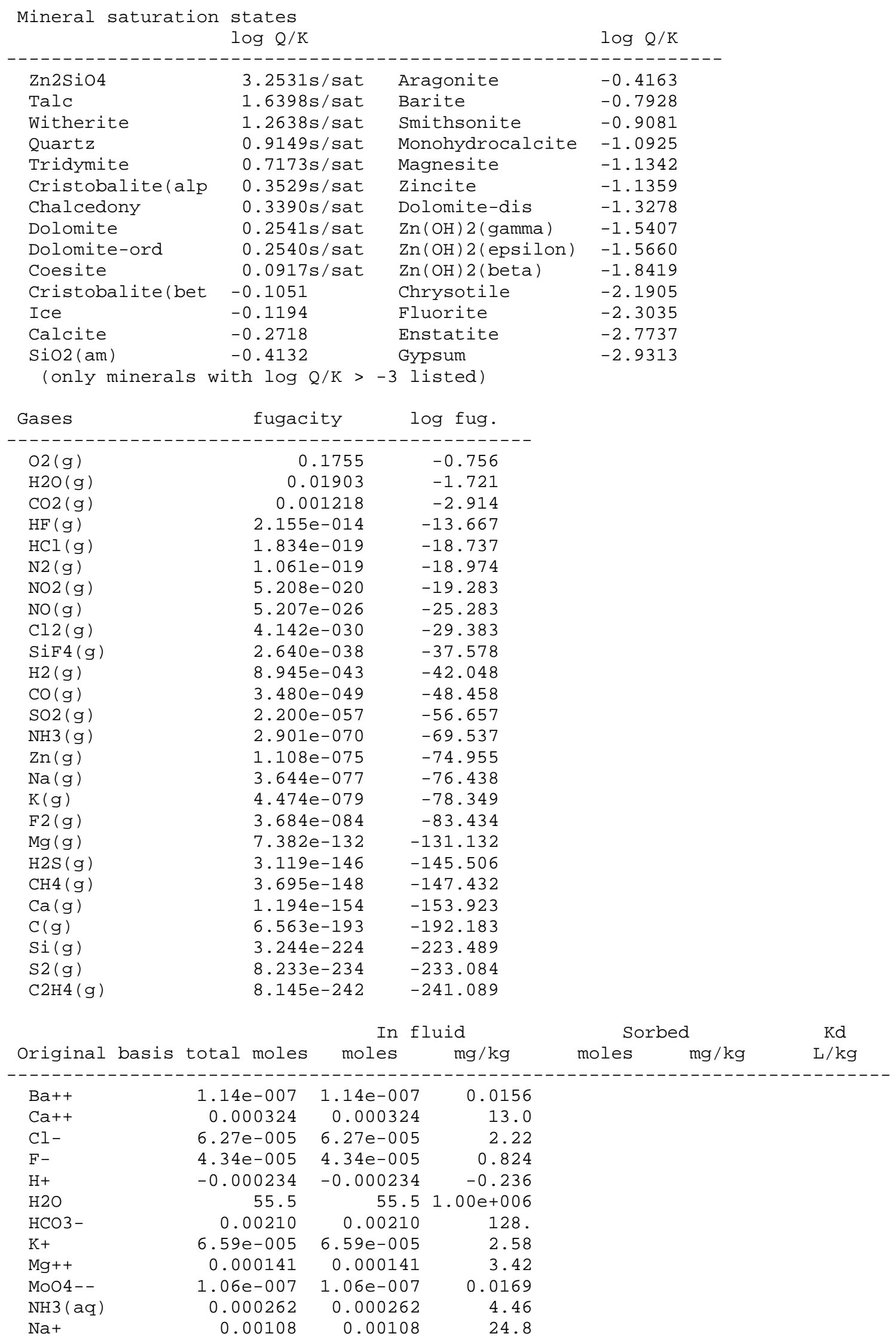




\begin{tabular}{|c|c|c|c|c|}
\hline $02(\mathrm{aq})$ & 0.000770 & 0.000770 & \multicolumn{2}{|l|}{24.6} \\
\hline $\mathrm{ReO4-}$ & $5.50 \mathrm{e}-006$ & $5.5 \odot \mathrm{e}-\odot \odot 6$ & \multicolumn{2}{|l|}{1.37} \\
\hline SO4 - - & $\odot .0 \odot \odot 2 \odot 5$ & & \multicolumn{2}{|l|}{19.7} \\
\hline $\operatorname{SiO} 2(\mathrm{aq})$ & $\odot .000613$ & 0.000613 & \multicolumn{2}{|l|}{36.8} \\
\hline $\mathrm{Zn++}$ & $3.12 \mathrm{e}-006$ & $3.12 \mathrm{e}-006$ & \multicolumn{2}{|l|}{$\odot .204$} \\
\hline \multicolumn{2}{|c|}{ Elemental composition } & \multicolumn{2}{|c|}{ In fluid } & Sorbed \\
\hline & total moles & moles & $\mathrm{mg} / \mathrm{kg}$ & moles $\quad \mathrm{mg} / \mathrm{kg}$ \\
\hline------- & 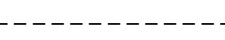 & 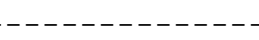 & 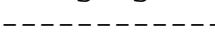 & 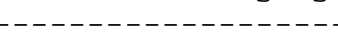 \\
\hline Barium & $1.139 \mathrm{e}-\odot \odot 7$ & $1.139 \mathrm{e}-007$ & 0.01564 & \\
\hline Calcium & $\odot .0 \odot \odot 3241$ & $\odot .0 \odot \odot 3241$ & 12.99 & \\
\hline Carbon & $\odot .0 \odot 2098$ & ๑. . 02098 & 25.19 & \\
\hline Chlorine & $6.273 e-005$ & $6.273 e-005$ & 2.224 & \\
\hline Fluorine & $4.341 e-0 \odot 5$ & $4.341 \mathrm{e}-005$ & 0.8245 & \\
\hline Hydrogen & 111.0 & 111.0 & $1.119 \mathrm{e}+0 \odot 5$ & \\
\hline Magnesium & ๑ ..$\odot \odot 14 \odot 9$ & ๑. .๑๑14०9 & 3.424 & \\
\hline Molybdenum & 1. $\odot 57 \mathrm{e}-\odot \odot 7$ & 1. $\odot 57 \mathrm{e}-\odot \odot 7$ & ๑. 01013 & \\
\hline Nitrogen & ๑. . $\odot \odot \odot 2619$ & ๑. . $00 \odot 2619$ & 3.667 & \\
\hline oxygen & 55.52 & 55.52 & $8.880 \mathrm{e}+\odot \odot 5$ & \\
\hline Potassium & $6.595 \mathrm{e}-0 \odot 5$ & $6.595 \mathrm{e}-0 \odot 5$ & 2.578 & \\
\hline Rhenium & $5.497 e-0 \odot 6$ & $5.497 e-006$ & 1.023 & \\
\hline Silicon & ๑. .0००6132 & ๑. .0००6132 & 17.22 & \\
\hline Sodium & 0.001079 & 0.001079 & 24.79 & \\
\hline Sulfur & $\odot .0 \odot \odot 2 \odot 49$ & ๑. .๑९2९49 & 6.568 & \\
\hline Zinc & $3.115 e-\odot \odot 6$ & $3.115 \mathrm{e}-\odot \odot 6$ & 0.2037 & \\
\hline
\end{tabular}




\section{Sample D14SB0T}

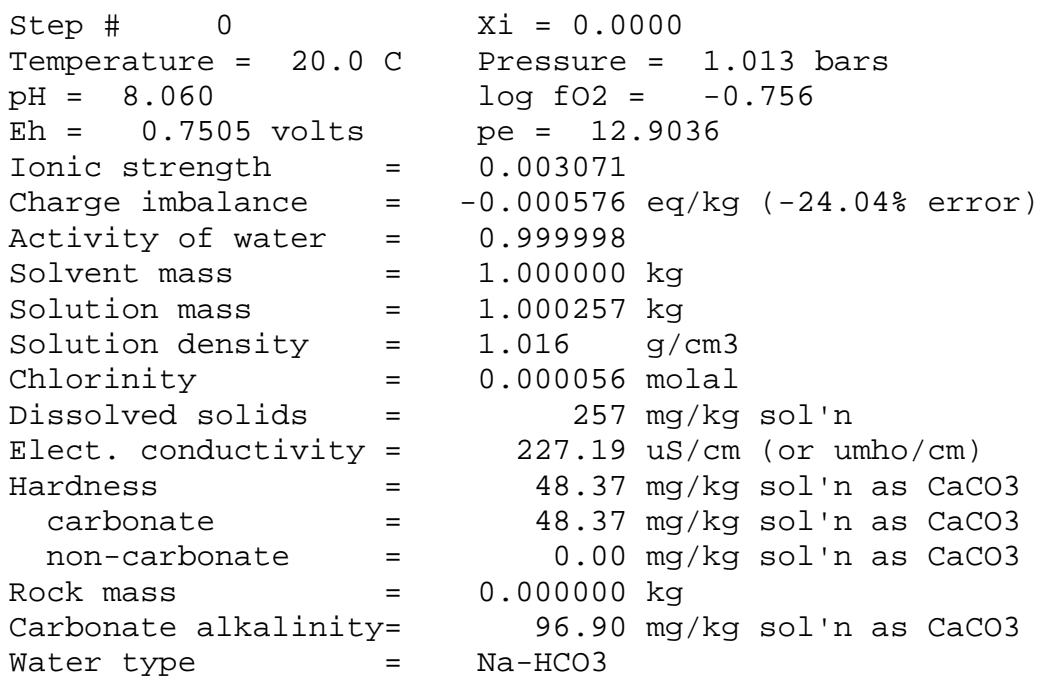

No minerals in system.

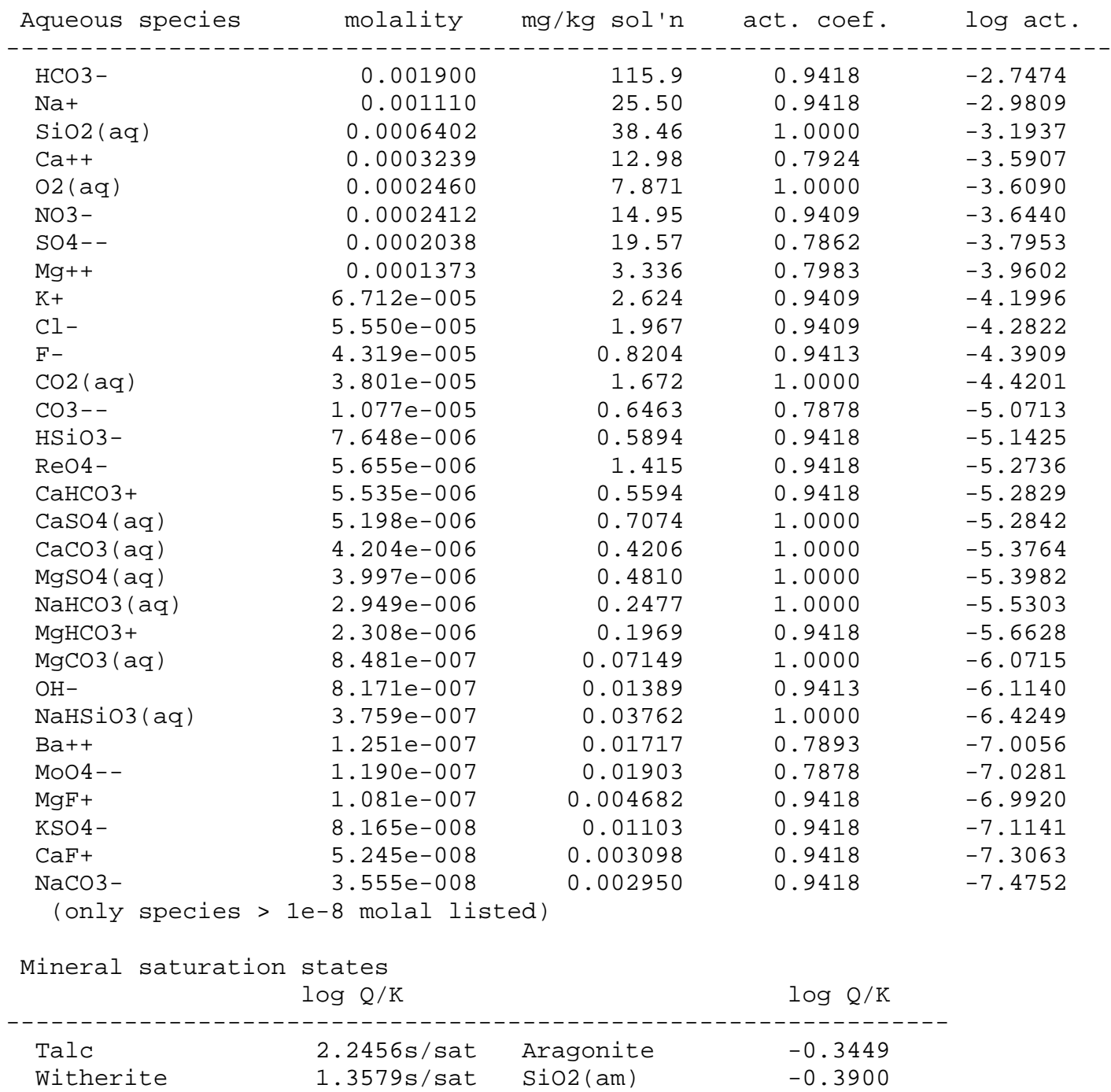




\begin{tabular}{|c|c|c|c|c|c|}
\hline Quartz & \multicolumn{2}{|c|}{$\odot .9381 \mathrm{~s} / \mathrm{sat}$} & Barite & \multicolumn{2}{|l|}{-0.7344} \\
\hline Tridymite & \multicolumn{2}{|c|}{$0.7405 \mathrm{~s} / \mathrm{sat}$} & Monohydrocalcite & -1.0211 & \\
\hline Dolomite & \multicolumn{2}{|c|}{$\odot .3889 \mathrm{~s} / \mathrm{sat}$} & Magnesite & -1.0708 & \\
\hline Dolomite-ord & \multicolumn{2}{|c|}{$0.3889 \mathrm{~s} / \mathrm{sat}$} & Dolomite-dis & -1.1929 & \\
\hline Cristobalite(alp & \multicolumn{2}{|c|}{$0.3761 \mathrm{~s} / \mathrm{sat}$} & Chrysotile & -1.6312 & \\
\hline Chalcedony & \multicolumn{2}{|c|}{$0.3622 \mathrm{~s} / \mathrm{sat}$} & Tremolite & -1.9037 & \\
\hline Coesite & \multicolumn{2}{|c|}{$0.1149 \mathrm{~s} / \mathrm{sat}$} & Fluorite & -2.2854 & \\
\hline Cristobalite (bet & \multicolumn{2}{|c|}{$-\odot .0819$} & Enstatite & -2.5796 & \\
\hline Ice & -0.1194 & & Gypsum & -2.8956 & \\
\hline $\begin{array}{l}\text { Calcite } \\
\text { (only minerals }\end{array}$ & $\begin{array}{l}-0.2003 \\
\text { with } \log Q\end{array}$ & $2 / K>-3$ & listed) & & \\
\hline Gases & fugaci & ity & $\log$ fug. & & \\
\hline 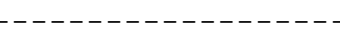 & -------- & $-\ldots$ & 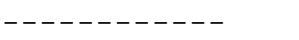 & & \\
\hline $02(g)$ & $\odot$ & 1755 & -0.756 & & \\
\hline $\mathrm{H} 2 \mathrm{O}(\mathrm{g})$ & 0.0 & 01903 & -1.721 & & \\
\hline $\mathrm{co2}(\mathrm{g})$ & $0.00 \odot$ & 09505 & -3.022 & & \\
\hline $\operatorname{HF}(g)$ & $1.790 \mathrm{e}$ & -014 & -13.747 & & \\
\hline $\mathrm{HCl}(\mathrm{g})$ & $1.350 \mathrm{e}$ & -019 & -18.870 & & \\
\hline $\mathrm{N} 2(\mathrm{~g})$ & $6.228 e$ & -020 & -19.206 & & \\
\hline N02 $(g)$ & $3.991 \mathrm{e}$ & -020 & -19.399 & & \\
\hline $\mathrm{NO}(\mathrm{g})$ & $3.990 \mathrm{e}$ & -026 & -25.399 & & \\
\hline Cl2(g) & $2.245 e$ & $2-030$ & -29.649 & & \\
\hline $\operatorname{SiF} 4(\mathrm{~g})$ & $1.327 e$ & -038 & -37.877 & & \\
\hline $\mathrm{H} 2(\mathrm{~g})$ & $8.945 e$ & -043 & -42.048 & & \\
\hline $\mathrm{CO}(\mathrm{g})$ & $2.716 \mathrm{e}$ & -049 & -48.566 & & \\
\hline so2(g) & $1.582 e$ & -057 & -56.801 & & \\
\hline $\mathrm{NH} 3(\mathrm{~g})$ & $2.223 e$ & -070 & -69.653 & & \\
\hline $\mathrm{Na}(\mathrm{g})$ & $4.521 \mathrm{e}$ & -077 & -76.345 & & \\
\hline $\mathrm{K}(\mathrm{g})$ & $5.482 e$ & -079 & -78.261 & & \\
\hline $\mathrm{F} 2(\mathrm{~g})$ & $2.543 e$ & -084 & -83.595 & & \\
\hline $\operatorname{Mg}(g)$ & $1.094 \mathrm{e}$ & -131 & -130.961 & & \\
\hline $\mathrm{H} 2 \mathrm{~S}(\mathrm{~g})$ & $2.242 e$ & -146 & -145.649 & & \\
\hline $\mathrm{CH} 4(\mathrm{~g})$ & $2.884 \mathrm{e}$ & -148 & -147.540 & & \\
\hline $\mathrm{Ca}(\mathrm{g})$ & $1.804 \mathrm{e}$ & -154 & -153.744 & & \\
\hline$C(g)$ & $5.123 e$ & -193 & -192.290 & & \\
\hline $\operatorname{si}(g)$ & $3.422 e$ & -224 & -223.466 & & \\
\hline $\mathrm{S} 2(\mathrm{~g})$ & $4.256 e$ & -234 & -233.371 & & \\
\hline $\mathrm{C} 2 \mathrm{H} 4(\mathrm{~g})$ & $4.962 e$ & -242 & -241.304 & & \\
\hline & & In & fluid & Sorbed & $\mathrm{Kd}$ \\
\hline Original basis tot & tal moles & moles & $\mathrm{mg} / \mathrm{kg}$ & moles $\quad \mathrm{mg} / \mathrm{kg}$ & $\mathrm{L} / \mathrm{kg}$ \\
\hline---1 & - - - - - & & & & \\
\hline $\mathrm{Ba}++$ & $.25 e-0 \odot 7$ & $1.25 \mathrm{e}-\odot \odot$ & 0.0172 & & \\
\hline $\mathrm{Ca}++$ & $\odot .0 \odot \odot 339$ & $\odot .00033$ & 13.6 & & \\
\hline $\mathrm{Cl}-$ & $.55 e-005$ & $5.55 \mathrm{e}-\odot \odot$ & 1.97 & & \\
\hline $\mathrm{F}-$ & $.34 \mathrm{e}-005$ & $4.34 \mathrm{e}-\odot \odot$ & 0.823 & & \\
\hline $\mathrm{H}+$ & $\odot .0 \odot \odot 228$ & $-\odot .0 \odot \odot 22$ & -0.230 & & \\
\hline $\mathrm{H} 2 \mathrm{O}$ & 55.5 & 55. & $.51 . \odot \odot \mathrm{e}+\odot \odot 6$ & & \\
\hline HCO3 - & 0.00196 & ๑. 0019 & $96 \quad 120$. & & \\
\hline $\mathrm{K}+$ & $.72 \mathrm{e}-\odot \odot 5$ & $6.72 \mathrm{e}-\odot \odot$ & 2.63 & & \\
\hline $\mathrm{Mg}++$ & $\odot .000145$ & 0.00014 & 3.51 & & \\
\hline Mo04 - - & $.19 \mathrm{e}-007$ & $1.19 \mathrm{e}-\odot \odot$ & 0.0190 & & \\
\hline $\mathrm{NH} 3(\mathrm{aq})$ & $\odot .0 \odot \odot 241$ & $\odot .00 \odot 24$ & 4.11 & & \\
\hline $\mathrm{Na}+$ & 0.00111 & 0.0011 & 25.6 & & \\
\hline $02(\mathrm{aq})$ & $\odot .0 \odot \odot 729$ & $\odot .0 \odot \odot 72$ & 23.3 & & \\
\hline Re04- & $.66 \mathrm{e}-0 \odot 6$ & $5.66 \mathrm{e}-\odot \odot$ & 1.41 & & \\
\hline SO4 - - & $\odot .000213$ & $\odot .00021$ & 20.5 & & \\
\hline Si02 (aq) & ๑. . 000648 & 0.00064 & 38.9 & & \\
\hline
\end{tabular}




\begin{tabular}{lrrr}
\multicolumn{2}{l}{ Elemental composition } & \multicolumn{2}{c}{ In fluid } \\
total moles & moles & mg/kg & Sorbed \\
Barium & $1.254 \mathrm{e}-007$ & $1.254 \mathrm{e}-007$ & 0.01722 \\
Calcium & 0.0003389 & 0.0003389 & 13.58 \\
Carbon & 0.001964 & 0.001964 & 23.59 \\
Chlorine & $5.552 \mathrm{e}-005$ & $5.552 \mathrm{e}-005$ & 1.968 \\
Fluorine & $4.336 \mathrm{e}-005$ & $4.336 \mathrm{e}-005$ & 0.8235 \\
Hydrogen & 111.0 & 111.0 & $1.119 \mathrm{e}+005$ \\
Magnesium & 0.0001446 & 0.0001446 & 3.512 \\
Molybdenum & $1.190 \mathrm{e}-007$ & $1.190 \mathrm{e}-007$ & 0.01141 \\
Nitrogen & 0.0002412 & 0.0002412 & 3.378 \\
Oxygen & 55.52 & 55.52 & $8.880 \mathrm{e}+005$ \\
Potassium & $6.720 \mathrm{e}-005$ & $6.720 \mathrm{e}-005$ & 2.627 \\
Rhenium & $5.655 \mathrm{e}-006$ & $5.655 \mathrm{e}-006$ & 1.053 \\
Silicon & 0.0006482 & 0.0006482 & 18.20 \\
Sodium & 0.001113 & 0.001113 & 25.58 \\
Sulfur & 0.0002131 & 0.0002131 & 6.831
\end{tabular}




\section{Sample D1419MMB_ts}

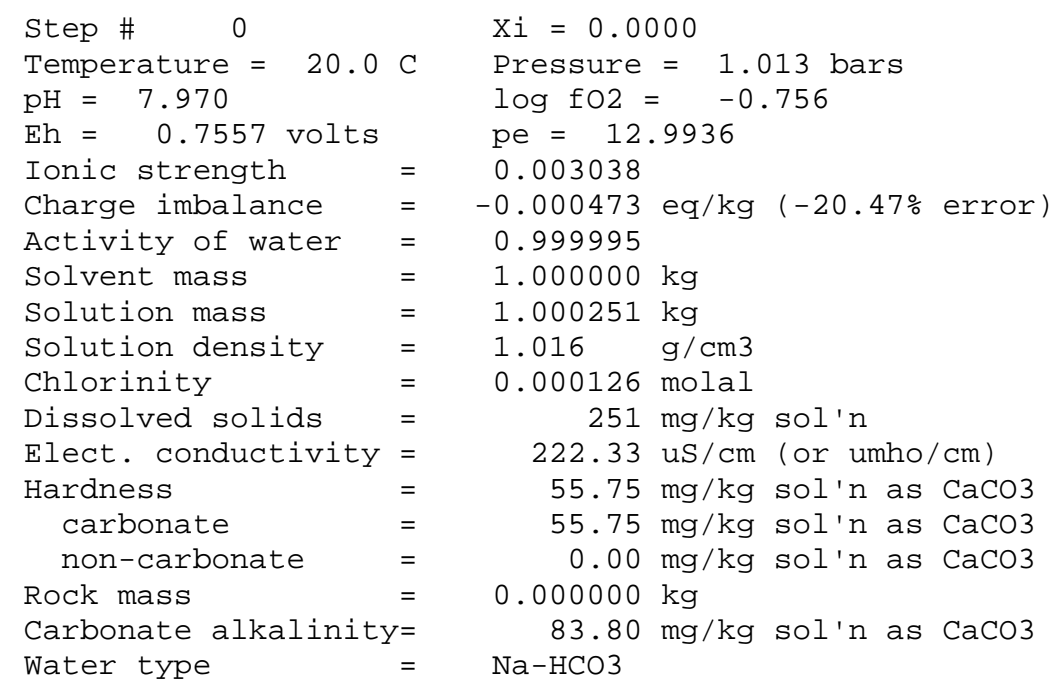

No minerals in system.

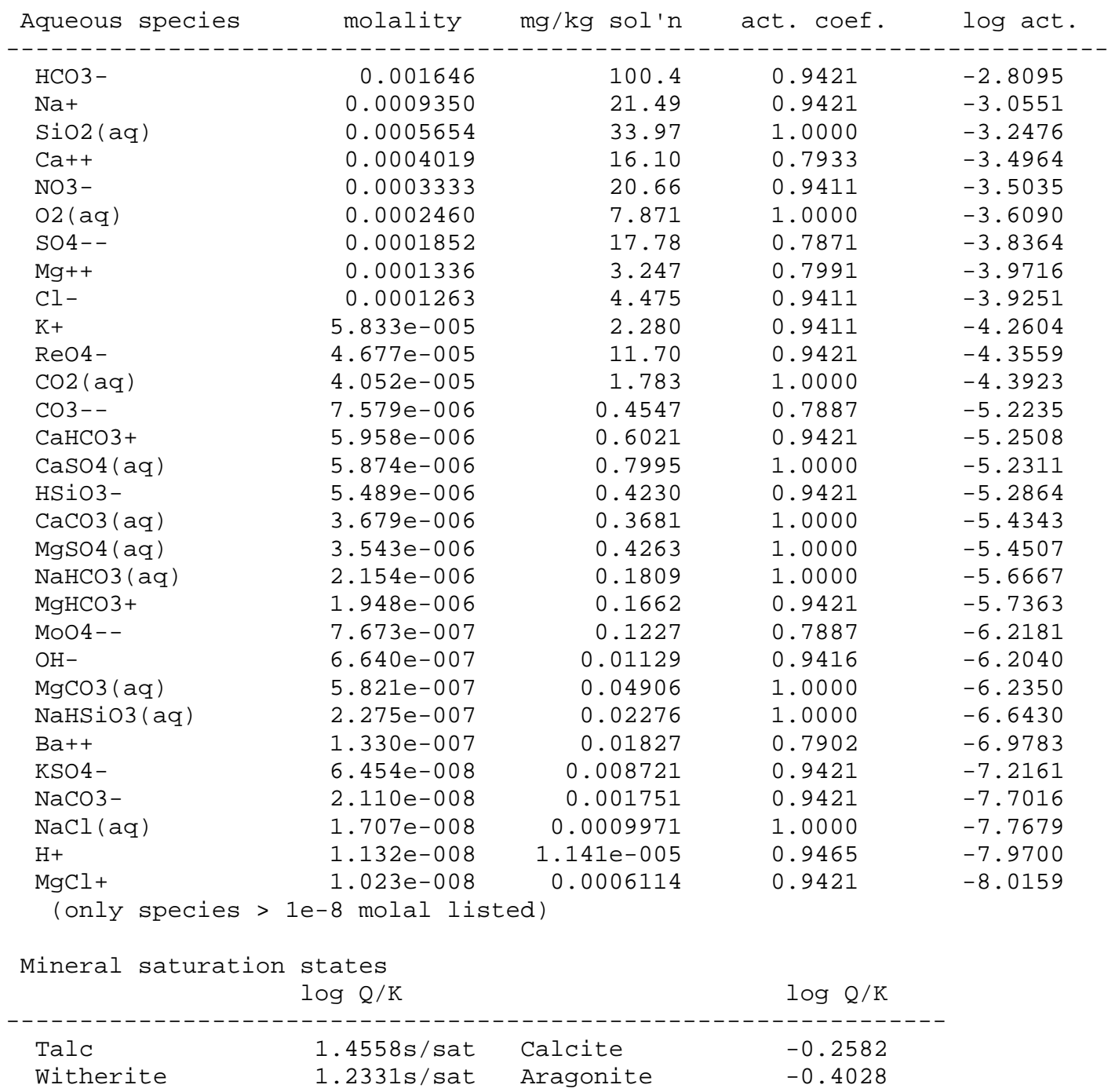




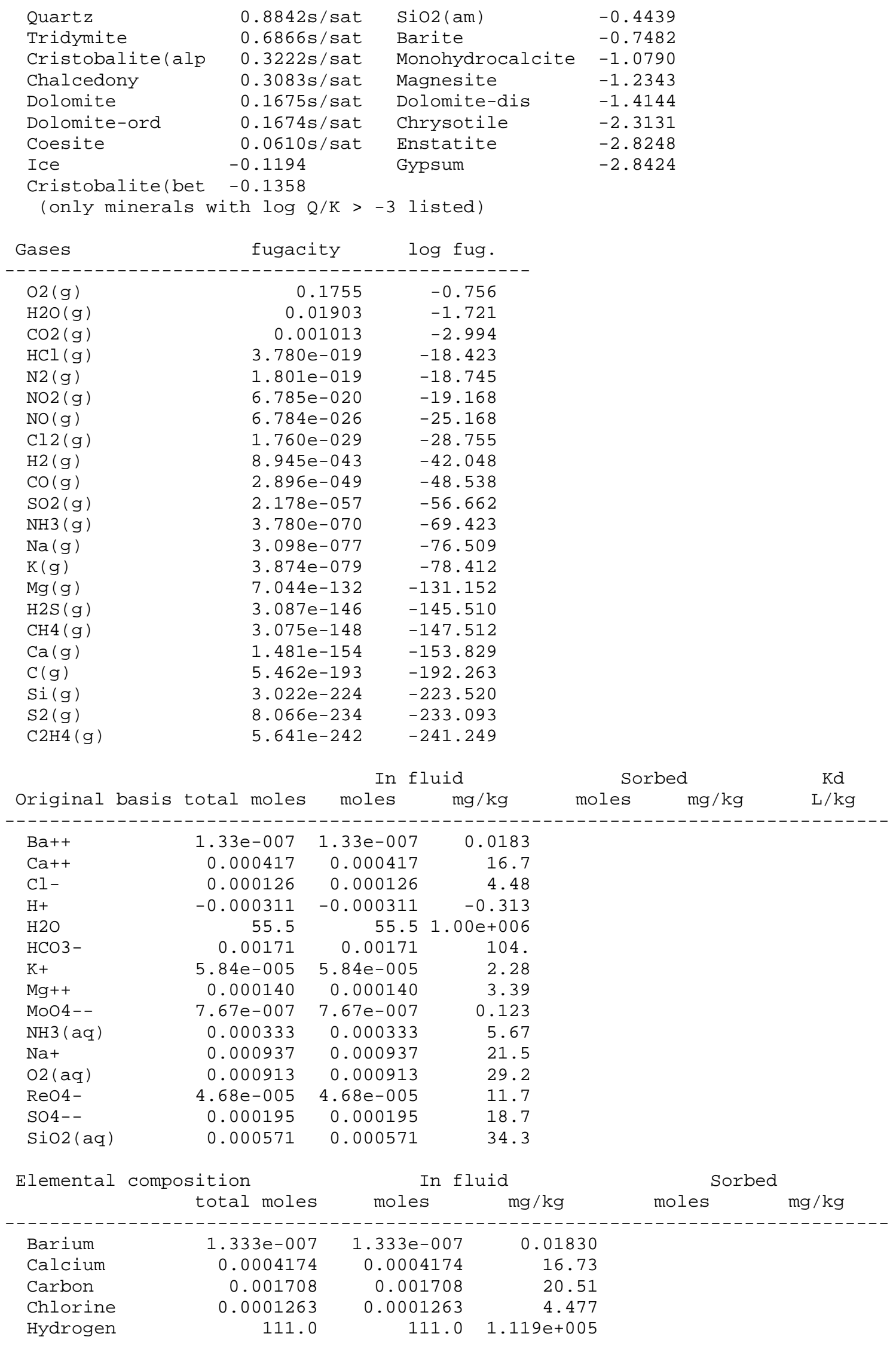




$\begin{array}{lrrr}\text { Magnesium } & 0.0001397 & 0.0001397 & 3.394 \\ \text { Molybdenum } & 7.673 \mathrm{e}-007 & 7.673 \mathrm{e}-0007 & 0.07359 \\ \text { Nitrogen } & 0.0003333 & 0.0003333 & 4.667 \\ \text { Oxygen } & 55.52 & 55.52 & 8.880 \mathrm{e}+005 \\ \text { Potassium } & 5.840 \mathrm{e}-005 & 5.840 \mathrm{e}-005 & 2.283 \\ \text { Rhenium } & 4.677 \mathrm{e}-005 & 4.677 \mathrm{e}-0005 & 8.707 \\ \text { Silicon } & 0.0005712 & 0.0005712 & 16.04 \\ \text { Sodium } & 0.0009375 & 0.0009375 & 21.55 \\ \text { Sulfur } & 0.0001946 & 0.0001946 & 6.240\end{array}$




\section{Sample D1419MMB_bs}

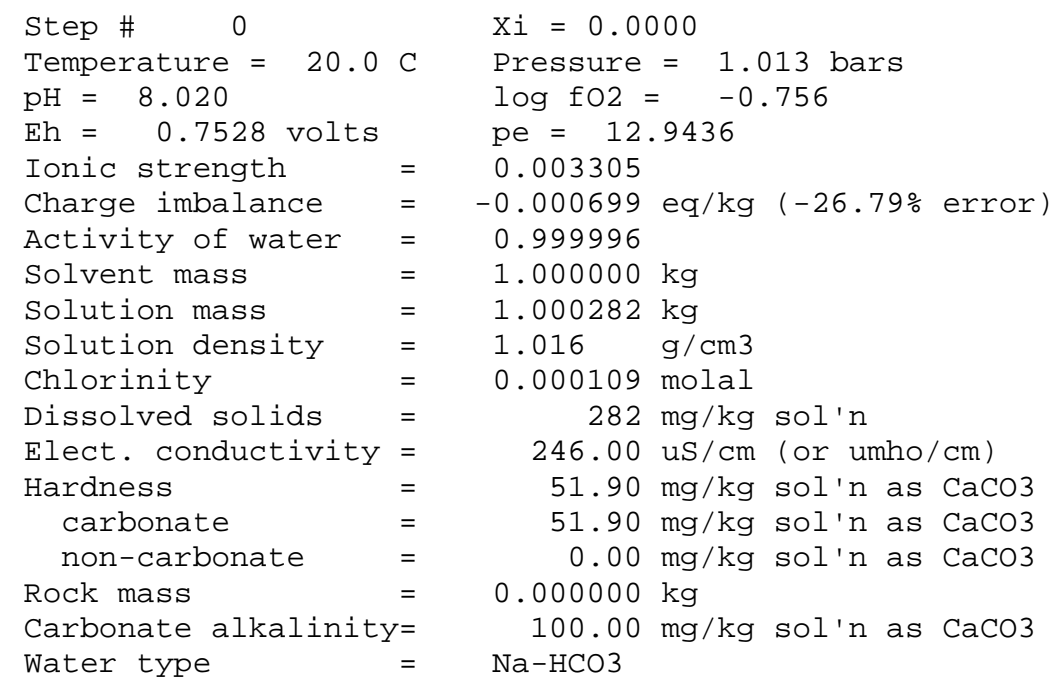

No minerals in system.

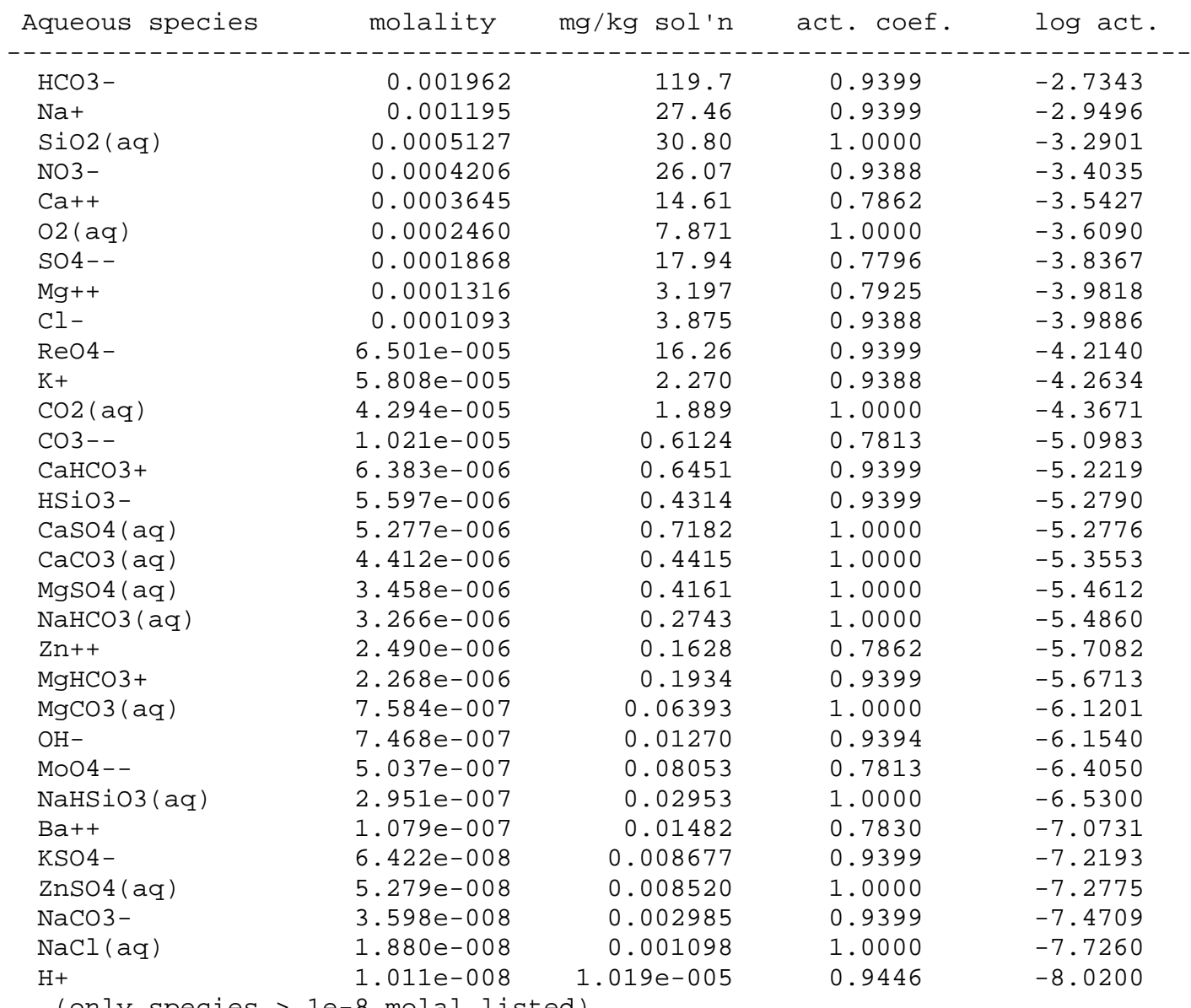

(only species $>1 e-8$ molal listed) 


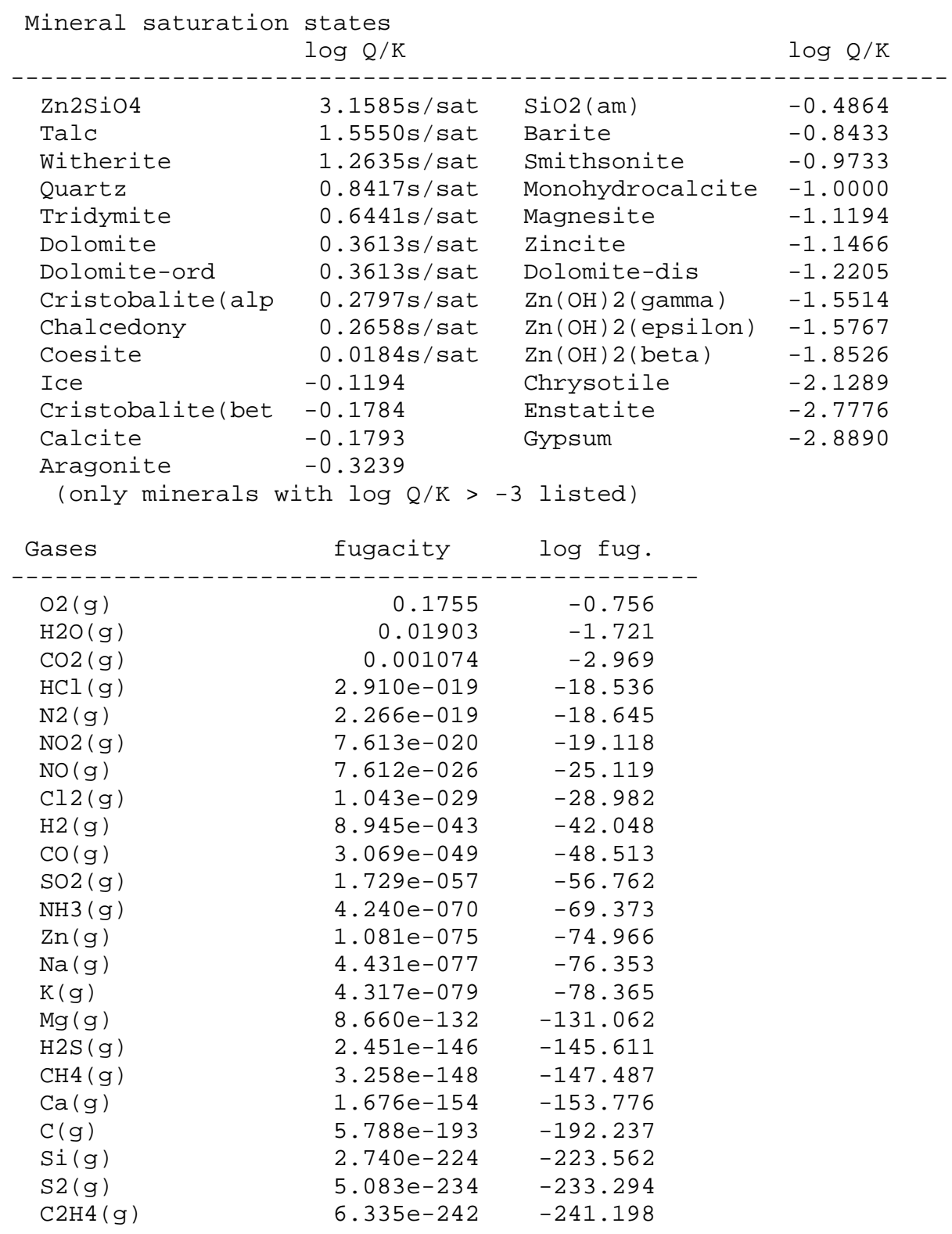




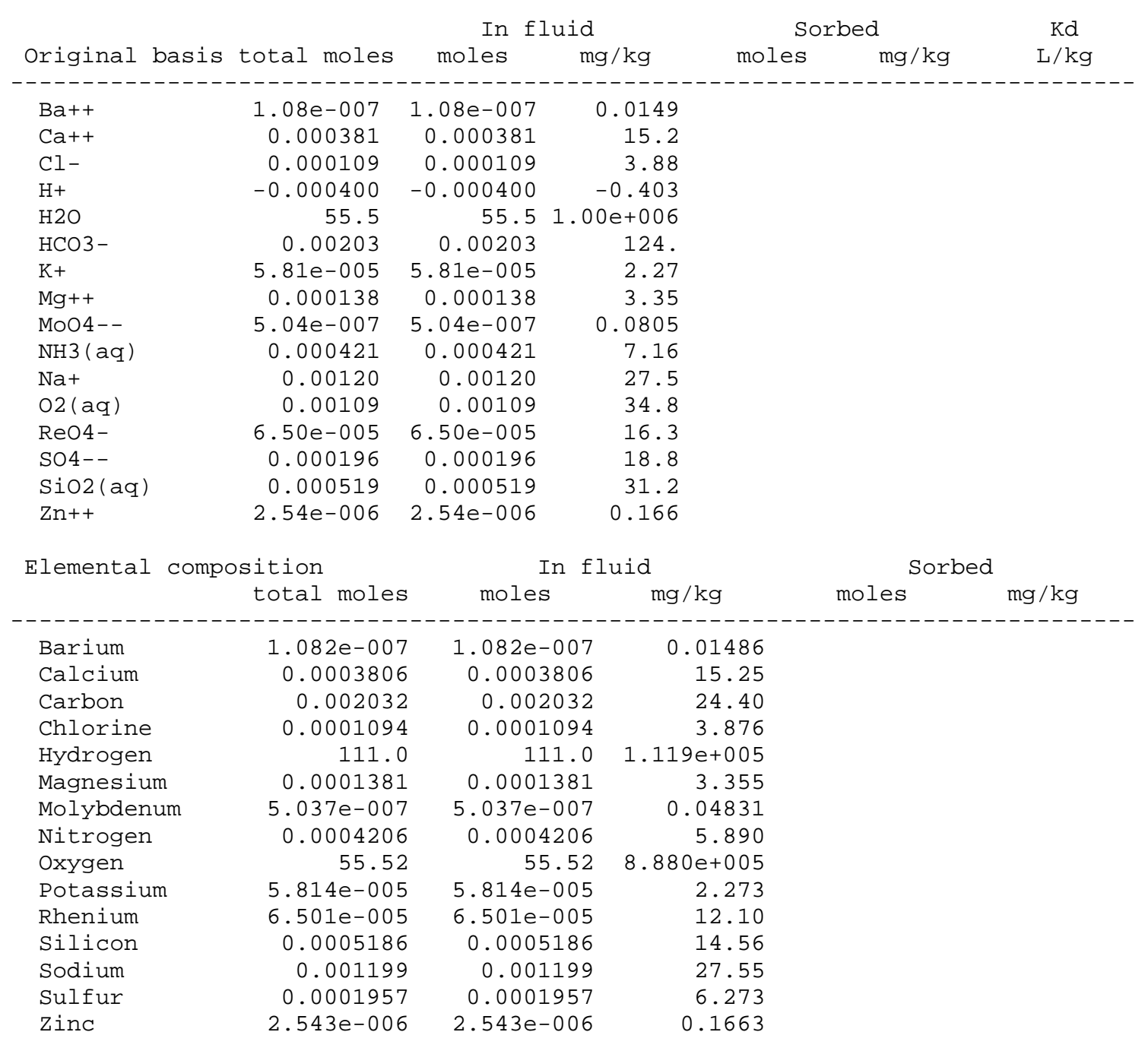




\section{Sample D14SEND}

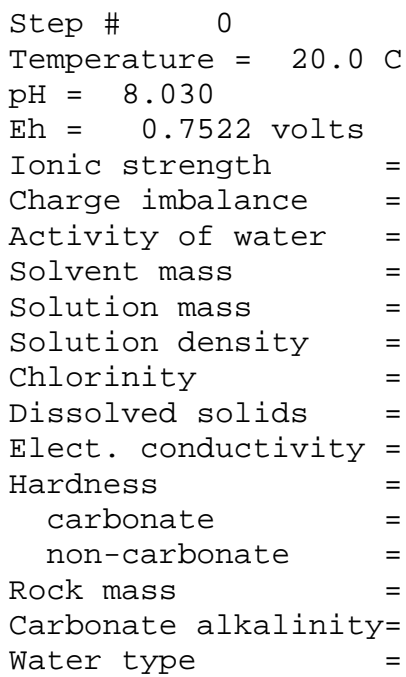

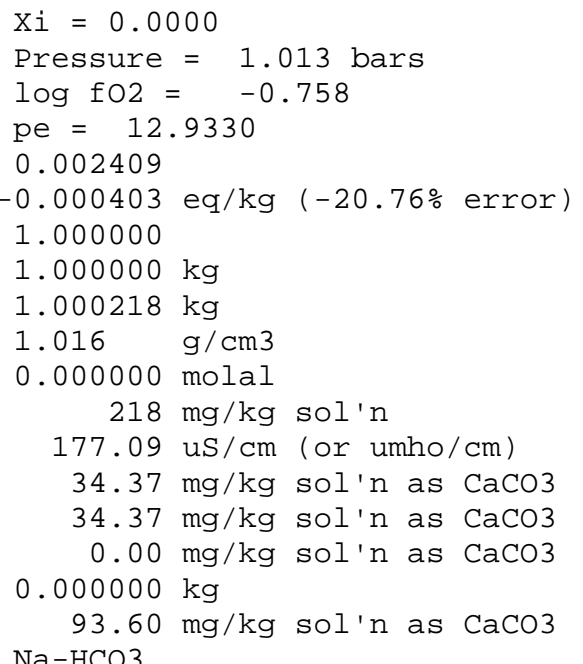

No minerals in system.

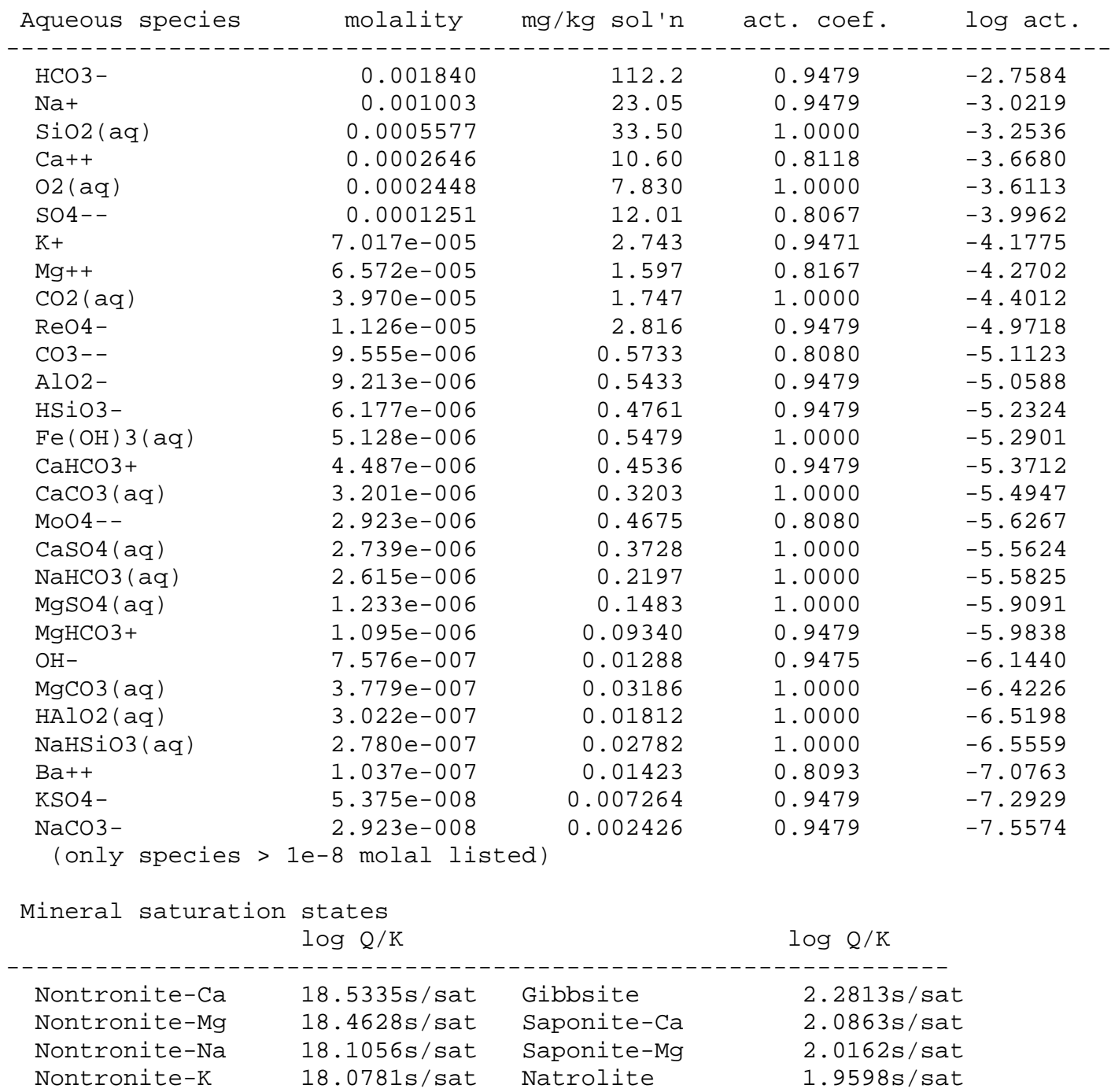




\begin{tabular}{|c|c|c|c|}
\hline Nontronite-H & $17.4700 \mathrm{~s} / \mathrm{sat}$ & Prehnite & $1.9390 \mathrm{~s} / \mathrm{sat}$ \\
\hline Clinoptilolite-h & $17.2887 \mathrm{~s} / \mathrm{sat}$ & Saponite-Na & $1.6584 \mathrm{~s} / \mathrm{sat}$ \\
\hline Clinoptilolite-C & $17.2597 \mathrm{~s} / \mathrm{sat}$ & Saponite-K & $1.6310 \mathrm{~s} / \mathrm{sat}$ \\
\hline Clinoptilolite-K & $13.5090 \mathrm{~s} / \mathrm{sat}$ & Corundum & $1.6076 \mathrm{~s} / \mathrm{sat}$ \\
\hline Clinoptilolite-h & $13.5034 \mathrm{~s} / \mathrm{sat}$ & Phlogopite & $1.5259 \mathrm{~s} / \mathrm{sat}$ \\
\hline Clinoptilolite-N & $13.5025 \mathrm{~s} / \mathrm{sat}$ & Clinozoisite & $1.5182 \mathrm{~s} / \mathrm{sat}$ \\
\hline Clinoptilolite-h & $13.3992 \mathrm{~s} / \mathrm{sat}$ & Zoisite & $1.4723 \mathrm{~s} / \mathrm{sat}$ \\
\hline Hematite & $12.9283 \mathrm{~s} / \mathrm{sat}$ & Albite_high & $1.4190 \mathrm{~s} / \mathrm{sat}$ \\
\hline Stilbite & $12.6115 \mathrm{~s} / \mathrm{sat}$ & Wairakite & $1.4048 \mathrm{~s} / \mathrm{sat}$ \\
\hline Muscovite & $10.9118 \mathrm{~s} / \mathrm{sat}$ & Witherite & $1.2461 \mathrm{~s} / \mathrm{sat}$ \\
\hline Epidote-ord & 8.4284s/sat & Magnetite & $1.1326 \mathrm{~s} / \mathrm{sat}$ \\
\hline Epidote & $8.4279 \mathrm{~s} / \mathrm{sat}$ & Kyanite & $1.1011 \mathrm{~s} / \mathrm{sat}$ \\
\hline Mesolite & $8.2592 \mathrm{~s} / \mathrm{sat}$ & Saponite-H & $1.0229 \mathrm{~s} / \mathrm{sat}$ \\
\hline Beidellite-Ca & $8.2504 \mathrm{~s} / \mathrm{sat}$ & Talc & $\odot .8958 \mathrm{~s} / \mathrm{sat}$ \\
\hline Illite & $8.2395 \mathrm{~s} / \mathrm{sat}$ & Clinochlore-14A & $\odot .8837 \mathrm{~s} / \mathrm{sat}$ \\
\hline Beidellite-Mg & $8.1797 \mathrm{~s} / \mathrm{sat}$ & Quartz & $\odot .8782 \mathrm{~s} / \mathrm{sat}$ \\
\hline Paragonite & $8.0324 \mathrm{~s} / \mathrm{sat}$ & Andalusite & $\odot .8175 \mathrm{~s} / \mathrm{sat}$ \\
\hline Beidellite-Na & $7.8224 \mathrm{~s} / \mathrm{sat}$ & Tridymite & $\odot .6806 \mathrm{~s} / \mathrm{sat}$ \\
\hline Beidellite-K & $7.7951 \mathrm{~s} / \mathrm{sat}$ & Sillimanite & $0.4441 \mathrm{~s} / \mathrm{sat}$ \\
\hline Herschelite & $7.7304 \mathrm{~s} / \mathrm{sat}$ & Cristobalite(alp & $0.3162 \mathrm{~s} / \mathrm{sat}$ \\
\hline Montmor-Mg & $7.5339 \mathrm{~s} / \mathrm{sat}$ & Chalcedony & $0.3023 \mathrm{~s} / \mathrm{sat}$ \\
\hline Montmor-Ca & $7.5332 \mathrm{~s} / \mathrm{sat}$ & Jadeite & $\odot .2692 \mathrm{~s} / \mathrm{sat}$ \\
\hline Beidellite-H & $7.1870 \mathrm{~s} / \mathrm{sat}$ & Coesite & $\odot .0550 \mathrm{~s} / \mathrm{sat}$ \\
\hline Montmor-Na & $7.1731 \mathrm{~s} / \mathrm{sat}$ & Dawsonite & $-\odot . \odot \odot 71$ \\
\hline Montmor-K & $7.1497 \mathrm{~s} / \mathrm{sat}$ & Dolomite & -0.0805 \\
\hline Pyrophyllite & $7.0629 \mathrm{~s} / \mathrm{sat}$ & Dolomite-ord & -0.0805 \\
\hline Kaolinite & $6.9827 \mathrm{~s} / \mathrm{sat}$ & Ice & -0.1194 \\
\hline Scolecite & $6.9240 \mathrm{~s} / \mathrm{sat}$ & Cristobalite(bet & -0.1418 \\
\hline Andradite & $6.6897 \mathrm{~s} / \mathrm{sat}$ & Kalsilite & -0.2474 \\
\hline Goethite & $5.9896 \mathrm{~s} / \mathrm{sat}$ & Analcime-K & -0.3083 \\
\hline Laumontite & $5.9753 \mathrm{~s} / \mathrm{sat}$ & Calcite & -0.3187 \\
\hline Celadonite & $5.4218 \mathrm{~s} / \mathrm{sat}$ & Si02(am) & -0.4499 \\
\hline Maximum_Microcli & $4.7413 \mathrm{~s} / \mathrm{sat}$ & Aragonite & -0.4632 \\
\hline K-Feldspar & $4.7401 \mathrm{~s} / \mathrm{sat}$ & Smectite-high-Fe & -0.4665 \\
\hline Margarite & $4.6919 \mathrm{~s} / \mathrm{sat}$ & Anorthite & $-\odot .8518$ \\
\hline Amesite-14A & $4.2322 \mathrm{~s} / \mathrm{sat}$ & Barite & -1.0061 \\
\hline Mordenite & 4.0692s/sat & Monohydrocalcite & -1.1394 \\
\hline Lawsonite & $3.6892 \mathrm{~s} / \mathrm{sat}$ & Analcime & -1.1990 \\
\hline Sanidine_high & $3.5082 \mathrm{~s} / \mathrm{sat}$ & Magnesite & -1.4219 \\
\hline Ferrite-Ca & $3.4948 \mathrm{~s} / \mathrm{sat}$ & Dolomite-dis & -1.6623 \\
\hline Ferrite-Mg & $3.3149 \mathrm{~s} / \mathrm{sat}$ & Nepheline & -2.0752 \\
\hline Diaspore & $2.8533 \mathrm{~s} / \mathrm{sat}$ & Clinochlore-7A & -2.5312 \\
\hline Albite_low & $2.7714 \mathrm{~s} / \mathrm{sat}$ & $\mathrm{Fe}(\mathrm{OH}) 3$ & -2.6982 \\
\hline Albite & $2.7713 \mathrm{~s} / \mathrm{sat}$ & Cordierite_hydr & -2.8043 \\
\hline Smectite-low-Fe- & $2.4924 \mathrm{~s} / \mathrm{sat}$ & Chrysotile & -2.8611 \\
\hline Boehmite & $2.4408 \mathrm{~s} / \mathrm{sat}$ & & \\
\hline (only minerals & vith $\log Q / K>$ & 3 listed) & \\
\hline Gases & fugacity & $\log$ fug. & \\
\hline.----- & $--_{-1}-\ldots$ & & \\
\hline $02(\mathrm{~g})$ & 0.1746 & -0.758 & \\
\hline $\mathrm{H} 2 \mathrm{O}(\mathrm{g})$ & 0.01903 & -1.721 & \\
\hline $\mathrm{CO} 2(\mathrm{~g})$ & ๑. 0009929 & -3.003 & \\
\hline $\mathrm{H} 2(\mathrm{~g})$ & $8.969 e-043$ & -42.047 & \\
\hline $\operatorname{co}(g)$ & $2.845 e-049$ & -48.546 & \\
\hline S02(g) & $1.147 \mathrm{e}-057$ & -56.941 & \\
\hline $\mathrm{Na}(\mathrm{g})$ & $3.843 e-077$ & -76.415 & \\
\hline$K(g)$ & $5.391 e-079$ & -78.268 & \\
\hline $\operatorname{Mg}(g)$ & $4.680 \mathrm{e}-132$ & -131.330 & \\
\hline $\mathrm{H} 2 \mathrm{~S}(\mathrm{~g})$ & $1.638 \mathrm{e}-146$ & -145.786 & \\
\hline $\mathrm{CH} 4(\mathrm{~g})$ & $3.044 \mathrm{e}-148$ & -147.517 & \\
\hline $\mathrm{Ca}(\mathrm{g})$ & $1.319 \mathrm{e}-154$ & -153.880 & \\
\hline $\mathrm{Al}(\mathrm{g})$ & $3.711 \mathrm{e}-192$ & -191.430 & \\
\hline
\end{tabular}




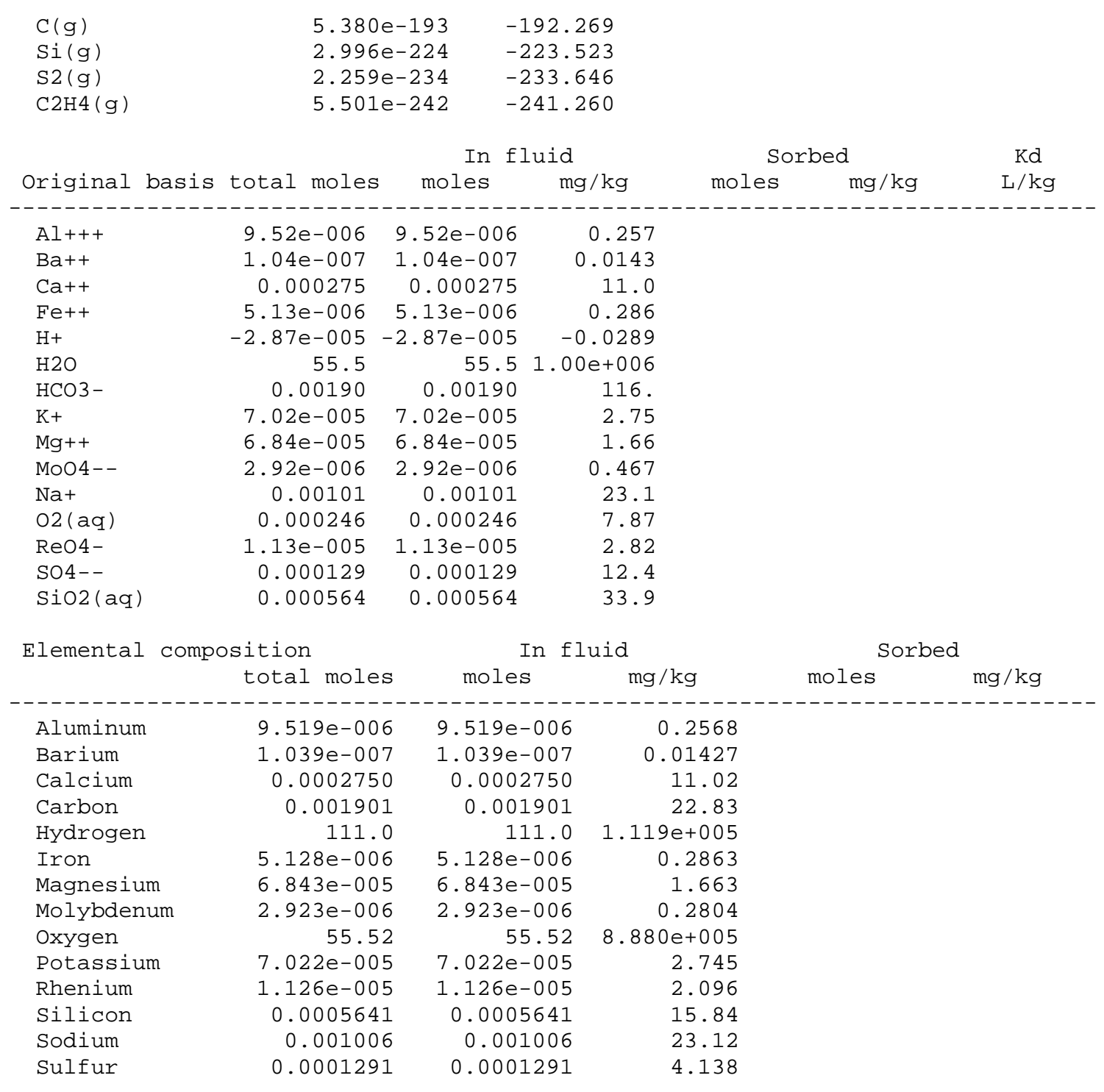




\section{Sample D14NTOP}

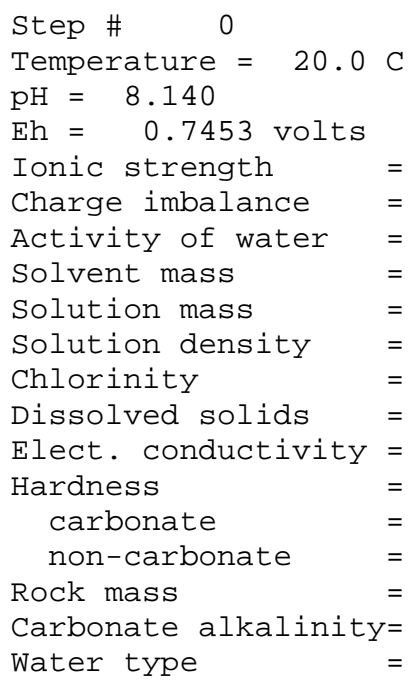

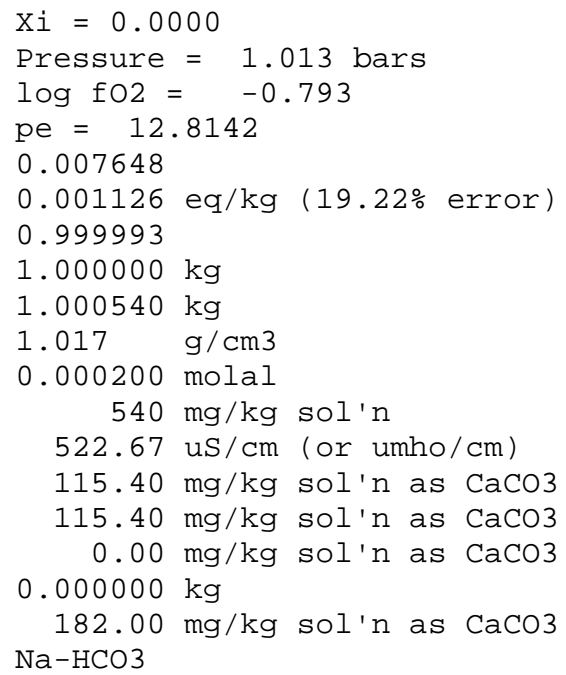

No minerals in system.

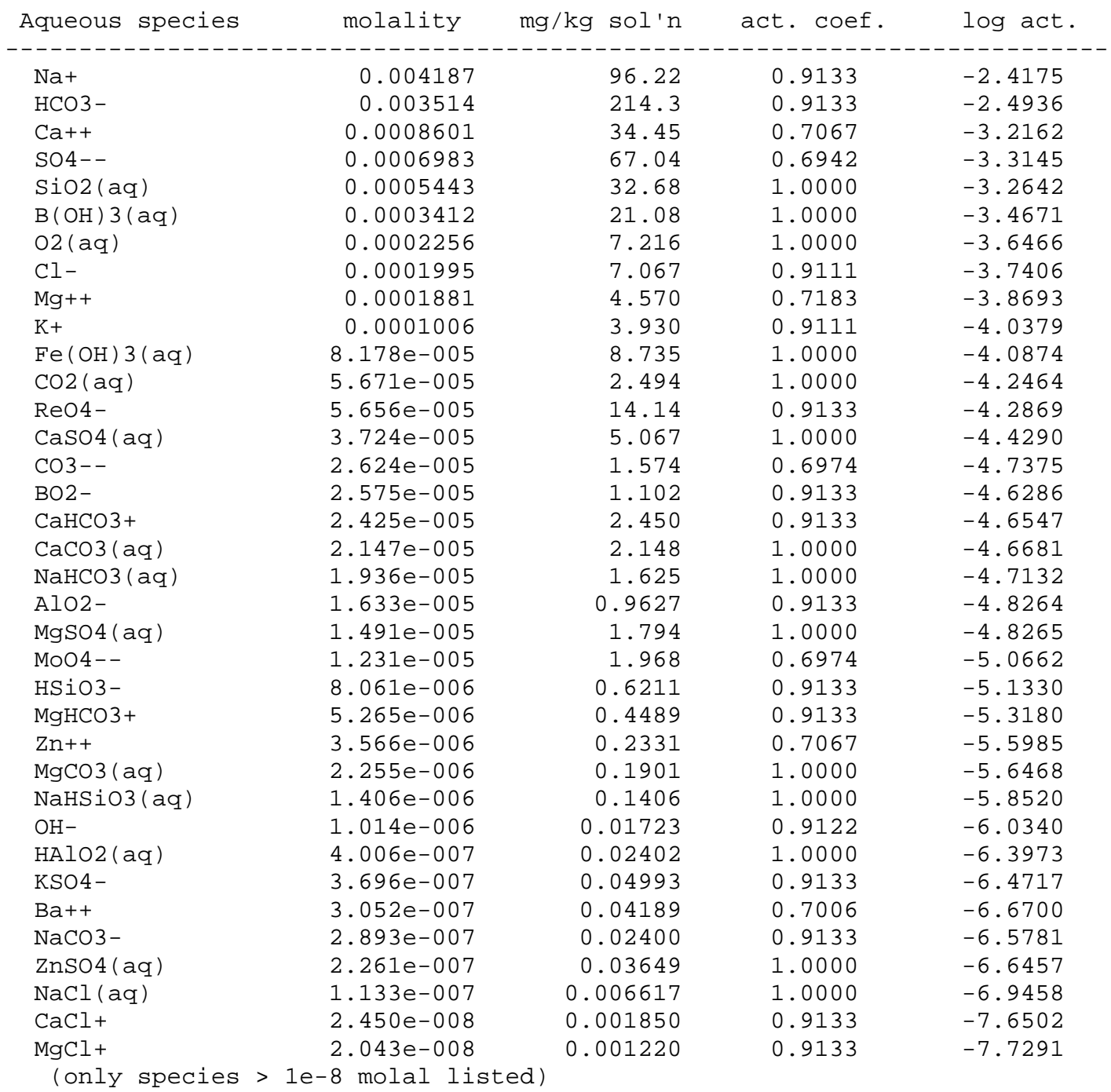




\begin{tabular}{|c|c|c|c|}
\hline Mineral saturatio & $\log \mathrm{Q} / \mathrm{K}$ & & $\log Q / K$ \\
\hline & & & \\
\hline Nontronite-Ca & $21.0513 \mathrm{~s} / \mathrm{sat}$ & Prehnite & $3.4956 \mathrm{~s} / \mathrm{sat}$ \\
\hline Nontronite-Mg & $20.9723 \mathrm{~s} / \mathrm{sat}$ & Clinozoisite & $3.1973 \mathrm{~s} / \mathrm{sat}$ \\
\hline Nontronite-Na & $20.7483 \mathrm{~s} / \mathrm{sat}$ & Zoisite & $3.1514 \mathrm{~s} / \mathrm{sat}$ \\
\hline Nontronite-K & $20.5674 \mathrm{~s} / \mathrm{sat}$ & Diaspore & $2.9757 \mathrm{~s} / \mathrm{sat}$ \\
\hline Nontronite-H & $19.8770 \mathrm{~s} / \mathrm{sat}$ & Saponite-H & $2.8874 \mathrm{~s} / \mathrm{sat}$ \\
\hline Clinoptilolite-h & $18.7422 \mathrm{~s} / \mathrm{sat}$ & Talc & $2.7164 \mathrm{~s} / \mathrm{sat}$ \\
\hline Clinoptilolite-C & $18.7132 \mathrm{~s} / \mathrm{sat}$ & Boehmite & $2.5633 \mathrm{~s} / \mathrm{sat}$ \\
\hline Clinoptilolite-h & $16.2695 \mathrm{~s} / \mathrm{sat}$ & Gibbsite & $2.4037 \mathrm{~s} / \mathrm{sat}$ \\
\hline Clinoptilolite-N & $16.2687 \mathrm{~s} / \mathrm{sat}$ & Wairakite & $2.2791 \mathrm{~s} / \mathrm{sat}$ \\
\hline Hematite & $15.3337 \mathrm{~s} / \mathrm{sat}$ & Albite_high & $2.2242 \mathrm{~s} / \mathrm{sat}$ \\
\hline Clinoptilolite-K & $14.6631 \mathrm{~s} / \mathrm{sat}$ & Witherite & $2.0273 \mathrm{~s} / \mathrm{sat}$ \\
\hline Clinoptilolite-h & $14.5532 \mathrm{~s} / \mathrm{sat}$ & Corundum & $1.8525 \mathrm{~s} / \mathrm{sat}$ \\
\hline Ferrite-Zn & $14.0943 \mathrm{~s} / \mathrm{sat}$ & Dolomite & $1.5218 \mathrm{~s} / \mathrm{sat}$ \\
\hline Stilbite & 13.5894s/sat & Dolomite-ord & $1.5218 \mathrm{~s} / \mathrm{sat}$ \\
\hline Muscovite & $11.4969 \mathrm{~s} / \mathrm{sat}$ & Smectite-high-Fe & $1.3481 \mathrm{~s} / \mathrm{sat}$ \\
\hline Epidote-ord & $11.1877 \mathrm{~s} / \mathrm{sat}$ & Kyanite & $1.3354 \mathrm{~s} / \mathrm{sat}$ \\
\hline Epidote & $11.1873 \mathrm{~s} / \mathrm{sat}$ & Jadeite & $1.0850 \mathrm{~s} / \mathrm{sat}$ \\
\hline Andradite & $11.0786 \mathrm{~s} / \mathrm{sat}$ & Andalusite & $1.0518 \mathrm{~s} / \mathrm{sat}$ \\
\hline Mesolite & $9.3953 \mathrm{~s} / \mathrm{sat}$ & Dawsonite & $\odot .9846 \mathrm{~s} / \mathrm{sat}$ \\
\hline Herschelite & $9.2470 \mathrm{~s} / \mathrm{sat}$ & Quartz & $\odot .8676 \mathrm{~s} / \mathrm{sat}$ \\
\hline Paragonite & $9.0825 \mathrm{~s} / \mathrm{sat}$ & Clinochlore-7A & $\odot .7868 \mathrm{~s} / \mathrm{sat}$ \\
\hline Illite & $8.7891 \mathrm{~s} / \mathrm{sat}$ & Sillimanite & $0.6784 \mathrm{~s} / \mathrm{sat}$ \\
\hline Beidellite-Ca & $8.6077 \mathrm{~s} / \mathrm{sat}$ & Tridymite & $0.6700 \mathrm{~s} / \mathrm{sat}$ \\
\hline Beidellite-Mg & $8.5286 \mathrm{~s} / \mathrm{sat}$ & Calcite & $0.5079 \mathrm{~s} / \mathrm{sat}$ \\
\hline Beidellite-Na & $8.3047 \mathrm{~s} / \mathrm{sat}$ & Aragonite & $0.3634 \mathrm{~s} / \mathrm{sat}$ \\
\hline Beidellite-K & $8.1238 \mathrm{~s} / \mathrm{sat}$ & Cristobalite(alp & $0.3056 \mathrm{~s} / \mathrm{sat}$ \\
\hline Montmor-Ca & 8.0111s/sat & Chalcedony & $\odot .2917 \mathrm{~s} / \mathrm{sat}$ \\
\hline Montmor-Mg & $8.0035 \mathrm{~s} / \mathrm{sat}$ & Kalsilite & $0.1140 \mathrm{~s} / \mathrm{sat}$ \\
\hline Scolecite & $7.8089 \mathrm{~s} / \mathrm{sat}$ & Barite & $0.0819 \mathrm{~s} / \mathrm{sat}$ \\
\hline Montmor- $\mathrm{Na}$ & $7.7760 \mathrm{~s} / \mathrm{sat}$ & Coesite & $0.0444 \mathrm{~s} / \mathrm{sat}$ \\
\hline Montmor -K & $7.5991 \mathrm{~s} / \mathrm{sat}$ & Anorthite & $0.0436 \mathrm{~s} / \mathrm{sat}$ \\
\hline Beidellite-H & $7.4334 \mathrm{~s} / \mathrm{sat}$ & Analcime-K & $\odot .0272 \mathrm{~s} / \mathrm{sat}$ \\
\hline Pyrophyllite & $7.2654 \mathrm{~s} / \mathrm{sat}$ & Dolomite-dis & -0.0600 \\
\hline Kaolinite & $7.2064 \mathrm{~s} / \mathrm{sat}$ & Ice & -0.1194 \\
\hline Goethite & $7.1923 \mathrm{~s} / \mathrm{sat}$ & Tremolite & -0.1442 \\
\hline Amesite-14A & $7.1847 \mathrm{~s} / \mathrm{sat}$ & Cristobalite(bet & -0.1524 \\
\hline Laumontite & $6.8496 \mathrm{~s} / \mathrm{sat}$ & Monohydrocalcite & -0.3128 \\
\hline Ferrite-Ca & $6.5720 \mathrm{~s} / \mathrm{sat}$ & Analcime & -0.4171 \\
\hline Celadonite & $6.3724 \mathrm{~s} / \mathrm{sat}$ & Si02 (am) & -0.4605 \\
\hline Ferrite-Mg & $6.3413 \mathrm{~s} / \mathrm{sat}$ & Smithsonite & -0.5030 \\
\hline Margarite & $5.8323 \mathrm{~s} / \mathrm{sat}$ & Magnesite & -0.6461 \\
\hline Maximum_Microcli & $5.0815 \mathrm{~s} / \mathrm{sat}$ & Zincite & -0.7970 \\
\hline K-Feldspar & $5.0803 \mathrm{~s} / \mathrm{sat}$ & Chrysotile & -1.0194 \\
\hline Magnetite & $4.7495 \mathrm{~s} / \mathrm{sat}$ & Cordierite_hydr & -1.1254 \\
\hline Lawsonite & $4.5847 \mathrm{~s} / \mathrm{sat}$ & $\mathrm{Zn}(\mathrm{OH}) 2($ gamma $)$ & -1.2017 \\
\hline Mordenite & $4.5831 \mathrm{~s} / \mathrm{sat}$ & $\mathrm{Zn}(\mathrm{OH}) 2$ (epsilon) & -1.2271 \\
\hline Clinochlore-14A & $4.2017 \mathrm{~s} / \mathrm{sat}$ & Nepheline & -1.2488 \\
\hline Saponite-Ca & $4.0617 \mathrm{~s} / \mathrm{sat}$ & $\mathrm{Fe}(\mathrm{OH}) 3$ & -1.4955 \\
\hline Saponite-Mg & $3.9832 \mathrm{~s} / \mathrm{sat}$ & $\mathrm{Zn}(\mathrm{OH}) 2$ (beta) & -1.5030 \\
\hline Zn2SiO4 & $3.8837 \mathrm{~s} / \mathrm{sat}$ & Antigorite & -1.5827 \\
\hline Smectite-low-Fe- & $3.8789 \mathrm{~s} / \mathrm{sat}$ & Gypsum & -2.0403 \\
\hline Sanidine_high & $3.8484 \mathrm{~s} / \mathrm{sat}$ & Anhydrite & -2.2652 \\
\hline Saponite-Na & $3.7587 \mathrm{~s} / \mathrm{sat}$ & Diopside & -2.3605 \\
\hline Phlogopite & $3.7290 \mathrm{~s} / \mathrm{sat}$ & Enstatite & -2.3991 \\
\hline Natrolite & $3.6019 \mathrm{~s} / \mathrm{sat}$ & Jarosite & -2.5407 \\
\hline Saponite-K & $3.5778 \mathrm{~s} / \mathrm{sat}$ & Grossular & -2.7985 \\
\hline Albite_low & $3.5765 \mathrm{~s} / \mathrm{sat}$ & Bassanite & -2.9116 \\
\hline & $3.5764 \mathrm{~s} / \mathrm{sat}$ & & \\
\hline (only miner & $h \log Q / K>$ & listed) & \\
\hline
\end{tabular}




\begin{tabular}{|c|c|c|c|c|c|}
\hline \multicolumn{5}{|c|}{ fugacity } & \\
\hline \multicolumn{6}{|c|}{ 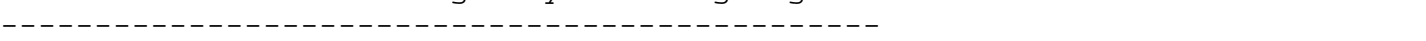 } \\
\hline $02(\mathrm{~g})$ & \multicolumn{2}{|c|}{0.1610} & -0.793 & & \\
\hline $\mathrm{H} 20(\mathrm{~g})$ & \multicolumn{2}{|c|}{$\odot .01903$} & -1.721 & & \\
\hline $\mathrm{CO} 2(\mathrm{~g})$ & \multicolumn{2}{|c|}{0.001418} & -2.848 & & \\
\hline $\mathrm{HCl}(\mathrm{g})$ & \multicolumn{2}{|c|}{$3.908 \mathrm{e}-019$} & -18.408 & & \\
\hline $\operatorname{cl} 2(\mathrm{~g})$ & \multicolumn{2}{|c|}{$1.801 \mathrm{e}-029$} & -28.744 & & \\
\hline $\mathrm{H} 2(\mathrm{~g})$ & \multicolumn{2}{|c|}{$9.341 \mathrm{e}-043$} & -42.030 & & \\
\hline $\mathrm{CO}(\mathrm{g})$ & \multicolumn{2}{|c|}{$4.232 \mathrm{e}-\odot 49$} & -48.373 & & \\
\hline S02(g) & $3.458 \mathrm{e}-$ & -057 & -56.461 & & \\
\hline $\mathrm{Zn}(\mathrm{g})$ & $2.526 \mathrm{e}-$ & -075 & -74.598 & & \\
\hline $\mathrm{Na}(\mathrm{g})$ & $2.032 \mathrm{e}-$ & -076 & -75.692 & & \\
\hline $\mathrm{K}(\mathrm{g})$ & $9.773 \mathrm{e}-$ & $-\odot 79$ & -78.010 & & \\
\hline $\operatorname{Mg}(g)$ & $2.037 e-$ & -131 & -130.691 & & \\
\hline $\mathrm{H} 2 \mathrm{~S}(\mathrm{~g})$ & $5.580 \mathrm{e}-$ & -146 & -145.253 & & \\
\hline $\mathrm{CH} 4(\mathrm{~g})$ & $5.116 \mathrm{e}-$ & -148 & -147.291 & & \\
\hline $\mathrm{Ca}(\mathrm{g})$ & $6.449 \mathrm{e}-$ & -154 & -153.190 & & \\
\hline $\mathrm{Al}(\mathrm{g})$ & $5.230 \mathrm{e}-$ & -192 & -191.282 & & \\
\hline$c(g)$ & $8.335 e-$ & -193 & -192.079 & & \\
\hline$B(g)$ & $6.669 \mathrm{e}-$ & -206 & -205.176 & & \\
\hline $\operatorname{si}(g)$ & $3.172 \mathrm{e}-$ & -224 & -223.499 & & \\
\hline $\mathrm{S} 2(\mathrm{~g})$ & $2.417 \mathrm{e}-$ & -233 & -232.617 & & \\
\hline $\mathrm{C} 2 \mathrm{H} 4(\mathrm{~g})$ & $1.432 \mathrm{e}-$ & -241 & -240.844 & & \\
\hline & & In & fluid & rbed & $\mathrm{Kd}$ \\
\hline Original basis & total moles & moles & $\mathrm{mg} / \mathrm{kg}$ & $\mathrm{mg} / \mathrm{kg}$ & $\mathrm{L} / \mathrm{kg}$ \\
\hline - - - - - - - - - & - - - - - - - - - - & $-\ldots-\ldots$ & - - - - - - - - - - - - - - - & 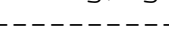 & $-\ldots$ \\
\hline $\mathrm{Al}+++$ & $1.67 \mathrm{e}-005 \quad 1$ & $1.67 \mathrm{e}-\odot \odot$ & $05 \quad 0.452$ & & \\
\hline $\mathrm{B}(\mathrm{OH}) 3(\mathrm{aq})$ & $\odot .0 \odot \odot 367$ & $\odot .00036$ & 22.7 & & \\
\hline $\mathrm{Ba}++$ & $3.07 e-007$ & $3.07 \mathrm{e}-\odot \odot$ & 0.0421 & & \\
\hline $\mathrm{Ca}++$ & $\odot .0 \odot \odot 943$ & $\odot .00094$ & 37.8 & & \\
\hline $\mathrm{Cl}-$ & $\odot .0 \odot \odot 2 \odot \odot$ & $\odot . \odot \odot \odot 2 \odot$ & 7.07 & & \\
\hline $\mathrm{Fe}++$ & $8.18 e-005$ & $8.18 \mathrm{e}-\odot \odot$ & 4.56 & & \\
\hline $\mathrm{H}+$ & -0.000260 & -0.00026 & $60 \quad-0.262$ & & \\
\hline $\mathrm{H} 2 \mathrm{O}$ & 55.5 & 55 & $.59 .99 \mathrm{e}+\odot \odot 5$ & & \\
\hline HCO3 - & $\odot .00367$ & $\odot .0036$ & $67 \quad 224$ & & \\
\hline $\mathrm{K}+$ & $\odot .000101$ & 0.00010 & 3.94 & & \\
\hline $\mathrm{Mg}++$ & $\odot .000211$ & $\odot .0 \odot \odot 21$ & 5.12 & & \\
\hline Mo04 - - & $1.23 e-005$ & $1.23 e-00$ & 1.97 & & \\
\hline $\mathrm{Na}+$ & 0.00421 & 0.0042 & 96.7 & & \\
\hline $02(\mathrm{aq})$ & $\odot .0 \odot \odot 246$ & $\odot .0 \odot \odot 24$ & 7.87 & & \\
\hline $\mathrm{ReO} 4$ - & $5.66 e-005$ & $5.66 e-00$ & 14.1 & & \\
\hline S04-- & $\odot .0 \odot \odot 751$ & $\odot .0 \odot 075$ & 72.1 & & \\
\hline Si02(aq) & $\odot .000554$ & $\odot .00055$ & 33.3 & & \\
\hline $\mathrm{Zn++}$ & $3.79 e-006$ & $3.79 \mathrm{e}-\odot \odot$ & 0.248 & & \\
\hline Elemental comp & sition & & In fluid & Sork & \\
\hline & total moles & mol & les $\quad \mathrm{mg} / \mathrm{kg}$ & moles & $\mathrm{mg} / \mathrm{kg}$ \\
\hline----------- & ---------- & ------ & 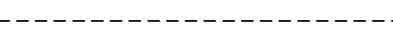 & --------6 & \\
\hline Aluminum & $1.674 \mathrm{e}-005$ & 1.674 & $4 e-005 \quad 0.4515$ & & \\
\hline Barium & $3.068 \mathrm{e}-007$ & 3.068 & 0.04210 & & \\
\hline Boron & $\odot .0003669$ & $\odot .0 \odot$ & ๑७3669 & & \\
\hline Calcium & 0.0009431 & $\odot .0 \odot$ & $0 \odot 9431$ & & \\
\hline Carbon & $\odot .0 \odot 3670$ & $\odot . \odot$ & ๑७3670 & & \\
\hline Chlorine & ๑ . 0001996 & $\odot .0 \odot$ & ๑1996 & & \\
\hline Hydrogen & 111.0 & & $1.118 \mathrm{e}+005$ & & \\
\hline Iron & $8.178 \mathrm{e}-\odot \odot 5$ & 8.178 & 4.565 & & \\
\hline Magnesium & 0.0002106 & $\odot .0 \odot$ & $0 \odot 2106$ & & \\
\hline Molybdenum & $1.231 \mathrm{e}-005$ & 1.231 & 1.180 & & \\
\hline oxygen & 55.53 & & $8.879 \mathrm{e}+005$ & & \\
\hline Potassium & $\odot .0 \odot 01009$ & 0.00 & 3.945 & & \\
\hline Rhenium & $5.656 e-005$ & 5.656 & $6 e-005$ & & \\
\hline
\end{tabular}


Silicon

Sodium

Sulfur

Zinc
๑. 0005537

๑. 004209

0.0007510

3. $793 \mathrm{e}-0 \odot 6$
๑. 0005537

0.004209

0.0007510

3. $793 \mathrm{e}-006$
15.54

96.70

24.07

$\odot .2479$

A. 137 


\section{Sample D14SEB0T}

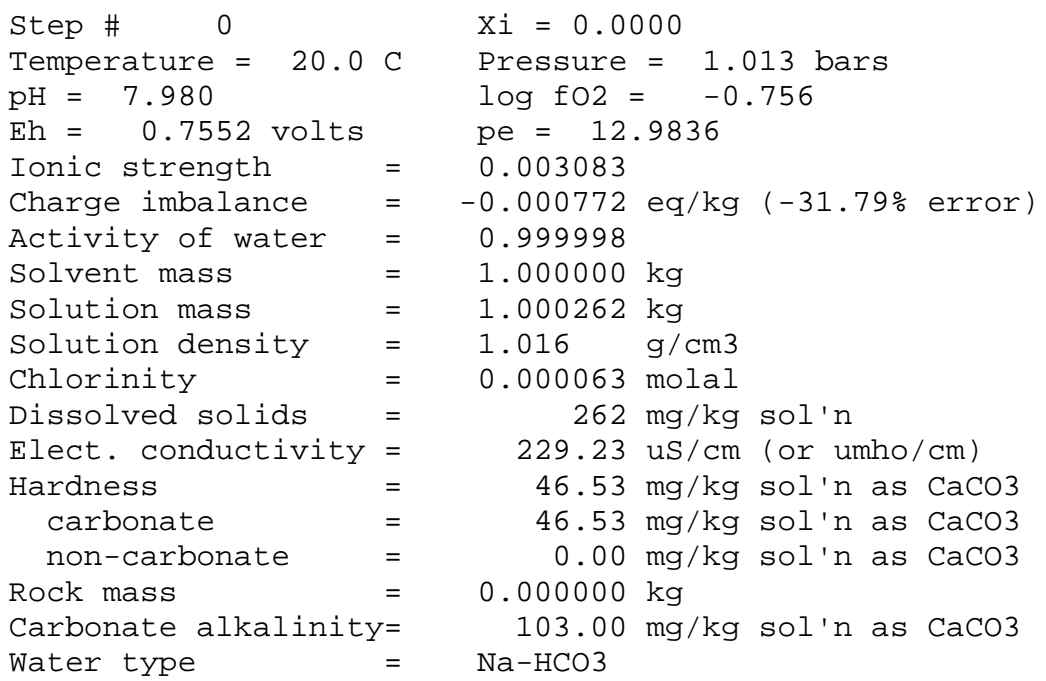

No minerals in system.

\begin{tabular}{|c|c|c|c|c|}
\hline Aqueous species & molality & $\mathrm{mg} / \mathrm{kg}$ sol'n & act. coef. & $\log$ act \\
\hline---------- & - - - - - - - - & - - - - - - - - - & - - - - - - - - & ---- \\
\hline $\mathrm{HCO} 3-$ & $\odot .0 \odot 2024$ & 123.5 & $\odot .9417$ & -2.7198 \\
\hline $\mathrm{Na}+$ & 0.001075 & 24.72 & $\odot .9417$ & -2.9945 \\
\hline $\operatorname{SiO} 2(\mathrm{aq})$ & 0.0006069 & 36.45 & 1.0000 & -3.2169 \\
\hline $\mathrm{Ca}++$ & ๑. . $00 \odot 3101$ & 12.42 & 0.7921 & -3.6098 \\
\hline N03 - & 0.0002619 & 16.23 & 0.9407 & -3.6084 \\
\hline $02(\mathrm{aq})$ & $\odot .0 \odot \odot 2460$ & 7.871 & $1.000 \odot$ & -3.6090 \\
\hline SO4 - - & $\odot .0001962$ & 18.84 & 0.7858 & -3.8120 \\
\hline $\mathrm{Mg}++$ & 0.0001339 & 3.254 & 0.7980 & -3.9712 \\
\hline $\mathrm{K}+$ & $6.587 e-0 \odot 5$ & 2.575 & 0.9407 & -4.2078 \\
\hline $\mathrm{Cl}-$ & $6.272 \mathrm{e}-0 \odot 5$ & 2.223 & 0.9407 & -4.2291 \\
\hline $\operatorname{co2}(\mathrm{aq})$ & $4.869 e-005$ & 2.142 & 1.0000 & -4.3125 \\
\hline $\mathrm{F}-$ & $4.325 e-0 \odot 5$ & $\odot .8214$ & $\odot .9412$ & -4.3903 \\
\hline CO3-- & $9.552 \mathrm{e}-006$ & 0.5731 & 0.7874 & -5.1237 \\
\hline HSi03 - & $6.031 e-006$ & 0.4648 & 0.9417 & -5.2457 \\
\hline $\mathrm{CaHCO}+$ & $5.645 e-006$ & 0.5706 & 0.9417 & -5.2744 \\
\hline Re04- & $5.497 e-006$ & 1.375 & 0.9417 & -5.2860 \\
\hline CaSO4(aq) & $4.787 e-0 \odot 6$ & $\odot .6515$ & 1.0000 & -5.3200 \\
\hline $\operatorname{MgSO} 4(\mathrm{aq})$ & $3.751 \mathrm{e}-006$ & 0.4514 & 1.0000 & -5.4259 \\
\hline $\mathrm{CaC0} 3(\mathrm{aq})$ & $3.566 e-006$ & 0.3568 & 1.0000 & -5.4478 \\
\hline $\mathrm{Zn++}$ & $3.046 e-0 \odot 6$ & $\odot .1991$ & $\odot .7921$ & -5.6175 \\
\hline $\mathrm{NaHCO} 3(\mathrm{aq})$ & $3.045 \mathrm{e}-006$ & 0.2558 & 1.0000 & -5.5164 \\
\hline $\mathrm{MgHCO} 3+$ & $2.399 e-0 \odot 6$ & 0.2046 & 0.9417 & -5.6461 \\
\hline $\operatorname{MgC0} 3(a q)$ & $7.330 \mathrm{e}-007$ & 0.06179 & 1.0000 & -6.1349 \\
\hline $\mathrm{OH}-$ & $6.797 \mathrm{e}-0 \odot 7$ & ๑. 01156 & 0.9412 & -6.1940 \\
\hline NaHSiO3 (aq) & $2.872 \mathrm{e}-0 \odot 7$ & 0.02874 & 1.0000 & -6.5418 \\
\hline $\mathrm{Ba}++$ & $1.137 \mathrm{e}-007$ & 0.01561 & 0.7890 & -7.0473 \\
\hline Mo04 - - & $1.057 \mathrm{e}-0 \odot 7$ & ๑. . 01689 & 0.7874 & -7.0799 \\
\hline $\mathrm{MgF}+$ & 1. $056 \mathrm{e}-007$ & 0.004571 & 0.9417 & -7.0025 \\
\hline KSO4 - & $7.710 \mathrm{e}-0 \odot 8$ & 0.01042 & 0.9417 & -7.1390 \\
\hline ZnSO4(aq) & $6.886 e-0 \odot 8$ & 0.01111 & 1.0000 & -7.1620 \\
\hline $\mathrm{CaF}+$ & $5.026 e-0 \odot 8$ & $\odot .0 \odot 2969$ & $\odot .9417$ & -7.3248 \\
\hline NaC03 - & $3.054 \mathrm{e}-008$ & ๑. .002534 & 0.9417 & -7.5413 \\
\hline $\mathrm{H}+$ & $1.107 \mathrm{e}-0 \odot 8$ & $1.115 \mathrm{e}-005$ & 0.9462 & -7.9800 \\
\hline
\end{tabular}




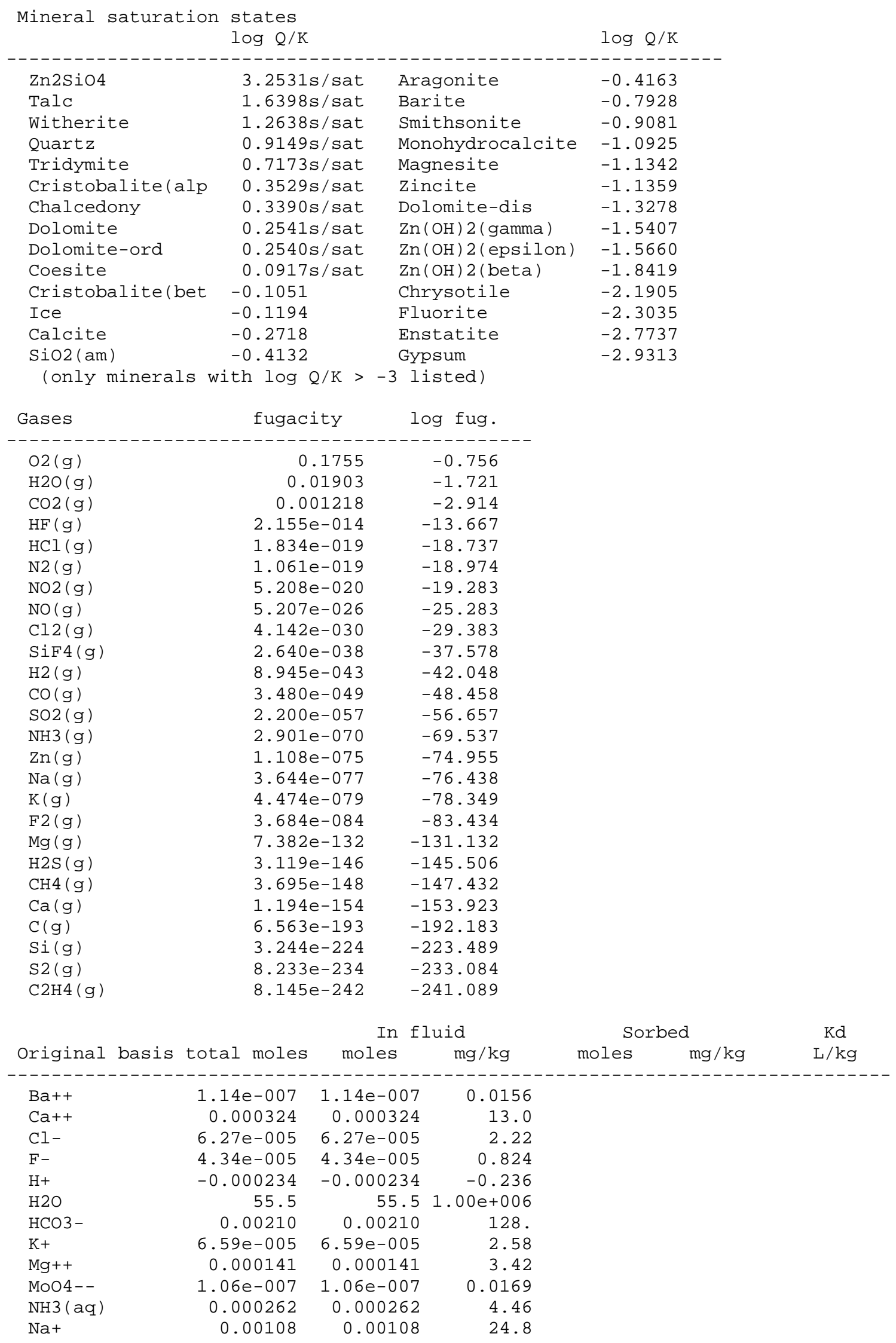




\begin{tabular}{|c|c|c|c|c|}
\hline $02(\mathrm{aq})$ & 0.000770 & 0.000770 & \multicolumn{2}{|l|}{24.6} \\
\hline $\mathrm{ReO4-}$ & $5.50 \mathrm{e}-006$ & $5.5 \odot \mathrm{e}-\odot \odot 6$ & \multicolumn{2}{|l|}{1.37} \\
\hline SO4 - - & $\odot .0 \odot \odot 2 \odot 5$ & & \multicolumn{2}{|l|}{19.7} \\
\hline $\operatorname{SiO} 2(\mathrm{aq})$ & $\odot .000613$ & 0.000613 & \multicolumn{2}{|l|}{36.8} \\
\hline $\mathrm{Zn++}$ & $3.12 \mathrm{e}-006$ & $3.12 \mathrm{e}-006$ & \multicolumn{2}{|l|}{$\odot .204$} \\
\hline \multicolumn{2}{|c|}{ Elemental composition } & \multicolumn{2}{|c|}{ In fluid } & Sorbed \\
\hline & total moles & moles & $\mathrm{mg} / \mathrm{kg}$ & moles $\quad \mathrm{mg} / \mathrm{kg}$ \\
\hline------- & 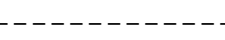 & 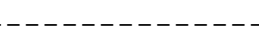 & 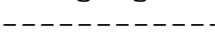 & 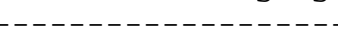 \\
\hline Barium & $1.139 \mathrm{e}-\odot \odot 7$ & $1.139 \mathrm{e}-007$ & 0.01564 & \\
\hline Calcium & $\odot .0 \odot \odot 3241$ & $\odot .0 \odot \odot 3241$ & 12.99 & \\
\hline Carbon & $\odot .0 \odot 2098$ & ๑. . 02098 & 25.19 & \\
\hline Chlorine & $6.273 e-005$ & $6.273 e-005$ & 2.224 & \\
\hline Fluorine & $4.341 e-0 \odot 5$ & $4.341 \mathrm{e}-005$ & 0.8245 & \\
\hline Hydrogen & 111.0 & 111.0 & $1.119 \mathrm{e}+0 \odot 5$ & \\
\hline Magnesium & ๑ ..$\odot \odot 14 \odot 9$ & ๑. .๑๑14०9 & 3.424 & \\
\hline Molybdenum & 1. $\odot 57 \mathrm{e}-\odot \odot 7$ & 1. $\odot 57 \mathrm{e}-\odot \odot 7$ & ๑. 01013 & \\
\hline Nitrogen & ๑. . $\odot \odot \odot 2619$ & ๑. . $00 \odot 2619$ & 3.667 & \\
\hline oxygen & 55.52 & 55.52 & $8.880 \mathrm{e}+\odot \odot 5$ & \\
\hline Potassium & $6.595 \mathrm{e}-0 \odot 5$ & $6.595 \mathrm{e}-0 \odot 5$ & 2.578 & \\
\hline Rhenium & $5.497 e-0 \odot 6$ & $5.497 e-006$ & 1.023 & \\
\hline Silicon & ๑. .0००6132 & ๑. .0००6132 & 17.22 & \\
\hline Sodium & 0.001079 & 0.001079 & 24.79 & \\
\hline Sulfur & $\odot .0 \odot \odot 2 \odot 49$ & ๑. .๑९2९49 & 6.568 & \\
\hline Zinc & $3.115 e-\odot \odot 6$ & $3.115 \mathrm{e}-\odot \odot 6$ & 0.2037 & \\
\hline
\end{tabular}


PNL-23693

RPT-66309-002, Rev. 0

\section{Distribution*}

Washington River Protection Solutions

DJ Swanberg

WRPS Documents - TOCVND@rl.gov

Oak Ridge National Laboratory

EM Pierce
Pacific Northwest National Laboratory

KJ Cantrell

VL Freedman

JJ Neeway

NP Qafoku

GL Smith

JH Westsik, Jr

Project File

Information Release (pdf)

*All distribution will be made electronically.

Dist. 1 



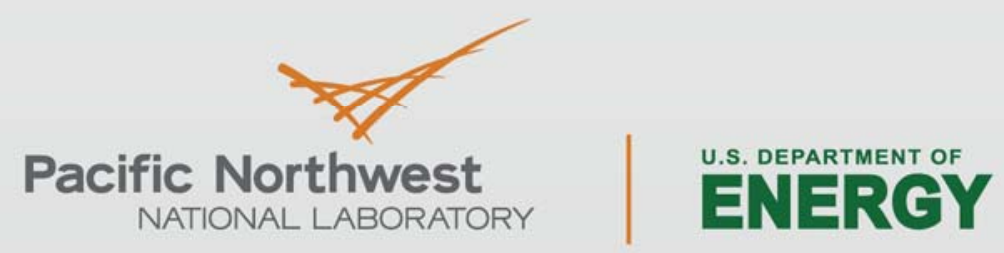

Proudly Operated by Battelle Since 1965

902 Battelle Boulevard

P.O. Box 999

Richland, WA 99352

1-888-375-PNNL (7665)

www.pnnl.gov 